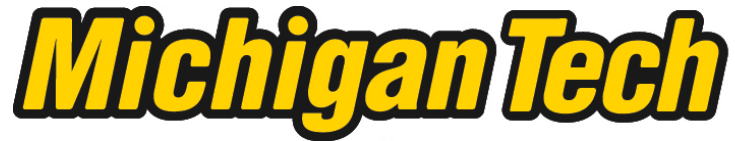 \\ Michigan Technological University Create the Future Digital Commons @ Michigan Tech
}

2015

BIO-INSPIRED OPTIMAL FIN SHAPE AND ANGLE FOR MAXIMUM SURFBOARD STABILITY

Megan S. MacNeill

Michigan Technological University

Follow this and additional works at: https://digitalcommons.mtu.edu/etds

Part of the Civil and Environmental Engineering Commons

Copyright 2015 Megan S. MacNeill

\section{Recommended Citation}

MacNeill, Megan S., "BIO-INSPIRED OPTIMAL FIN SHAPE AND ANGLE FOR MAXIMUM SURFBOARD STABILITY", Master's Thesis, Michigan Technological University, 2015.

https://doi.org/10.37099/mtu.dc.etds/998

Follow this and additional works at: https://digitalcommons.mtu.edu/etds

3 Part of the Civil and Environmental Engineering Commons 


\title{
BIO-INSPIRED OPTIMAL FIN SHAPE AND ANGLE FOR MAXIMUM
} SURFBOARD STABILITY

\author{
By
}

Megan S. MacNeill

\begin{abstract}
A THESIS
Submitted in partial fulfillment of the requirements for the degree of MASTER OF SCIENCE

In Civil Engineering
\end{abstract} MICHIGAN TECHNOLOGICAL UNIVERSITY

2015

(C)2015 Megan MacNeill 

This thesis has been approved in partial fulfillment of the requirements for the Degree of MASTER OF SCIENCE in Civil Engineering.

Department of Civil and Environmental Engineering

Thesis Advisor: Dr. Brian Barkdoll

Committee Member: Dr. Pengfei Xue

Committee Member: $\quad$ Dr. V.C. Rao Komaravolu

Department Chair: $\quad$ Dr. David Hand 



\section{Table of Contents}

List of Figures...................................................................................................................10

List of Tables .............................................................................................................. 16

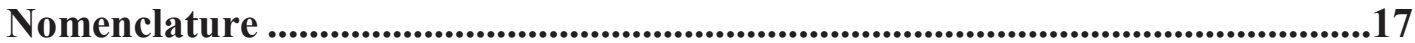

Acknowledgements .....................................................................................................19

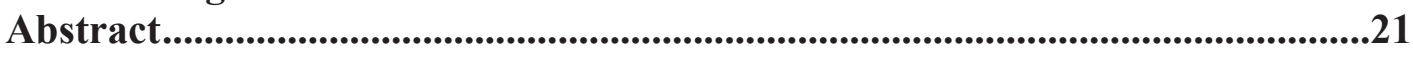

Chapter 1: Introduction and Motivation ......................................................................23

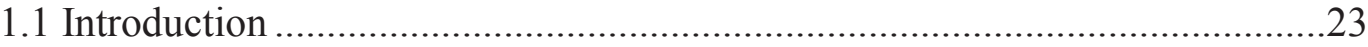

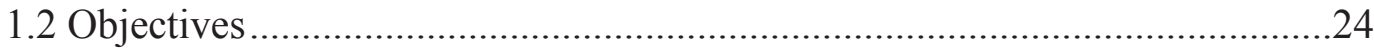

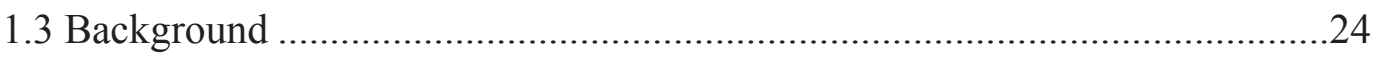

1.3.1 What is Wave Surfing? .....................................................................24

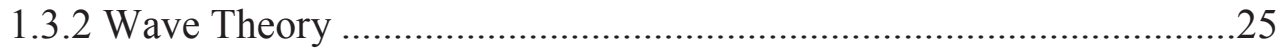

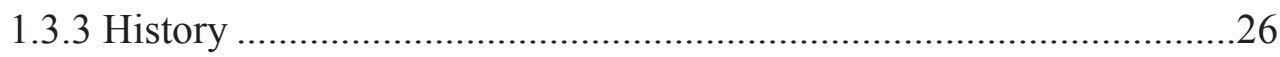

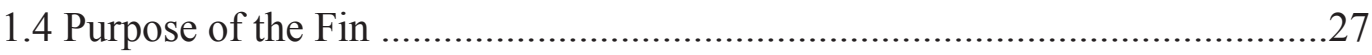

1.4.1 Skeg and Keel ………...................................................................27

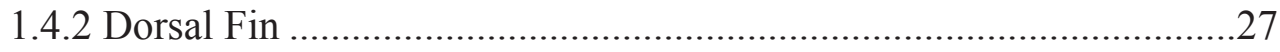

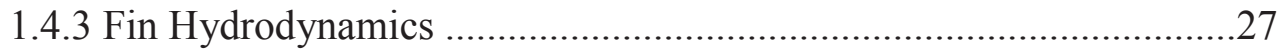

1.4.4 NACA Airfoils....................................................................................29

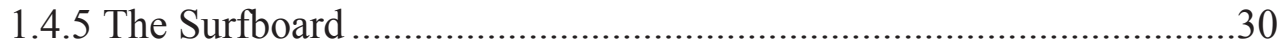

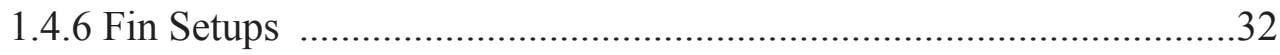

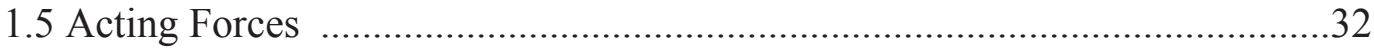

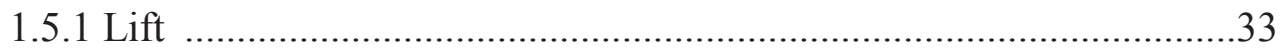

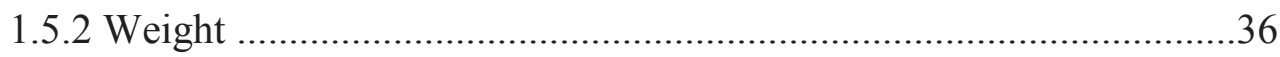

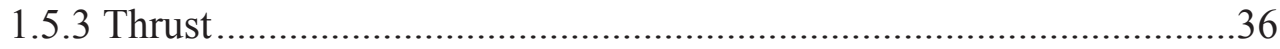

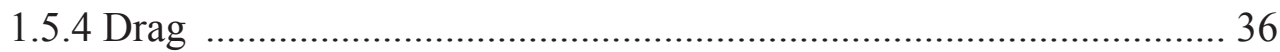

1.5.5 Lift and Drag Coefficients ..................................................................38

1.5.6 Turbulence Modeling and Boundary Effects......................................39

1.5.7 Near Wall Treatment .......................................................................

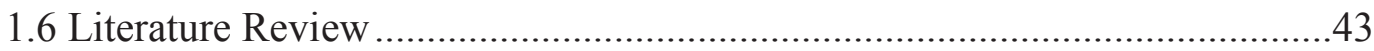

1.6.1 Surfboard Hydrodynamics ..................................................................43

1.7 Bio-Inspired Dorsal Fin Profiles ...................................................................45 


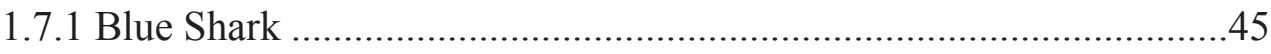

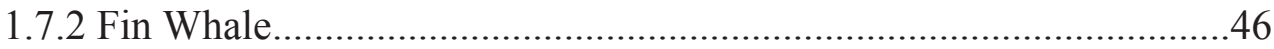

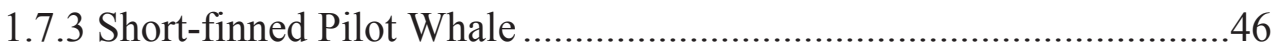

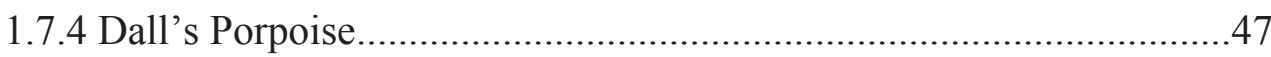

1.7.5 Pantropical Spotted Dolphin ..............................................................47

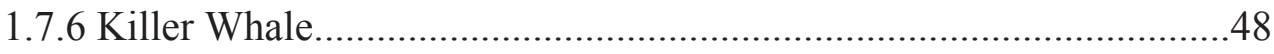

1.7.7 Shortfin Mako Shark.........................................................................49

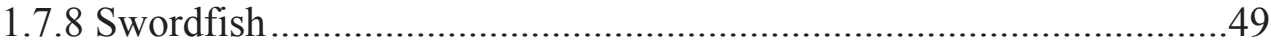

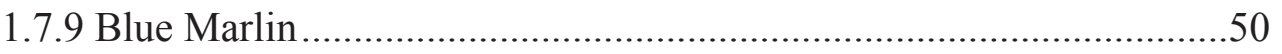

Chapter 2: CFD Experimental Setup..........................................................................51

2.1 Operational System …………………………………….............................

2.2 Modeling Programs ……………………………………............................

2.2.1 Microsoft Excel ...............................................................................

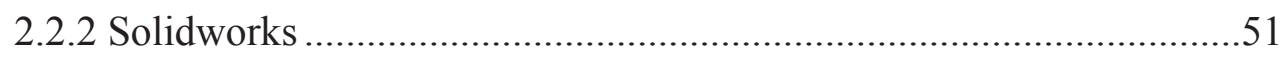

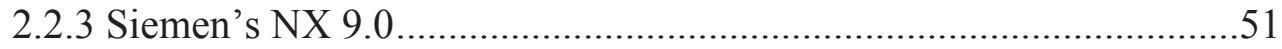

2.2.4 Turbulence Modeling............................................................................52

Chapter 3: Laboratory Experimental Setup ..........................................................53

3.1 Hydrodynamic Laboratory ………………………………...............................

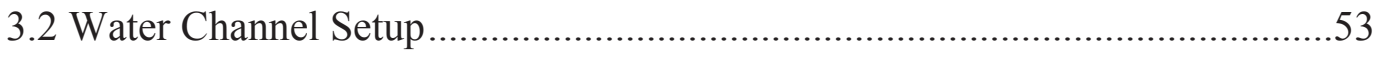

3.2.1 Water Channel Features....................................................................53

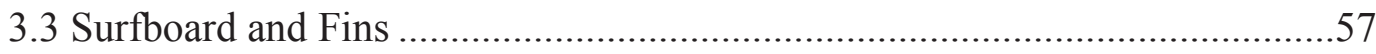

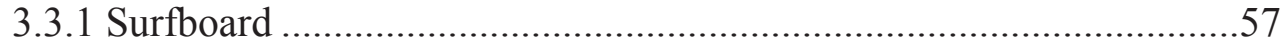

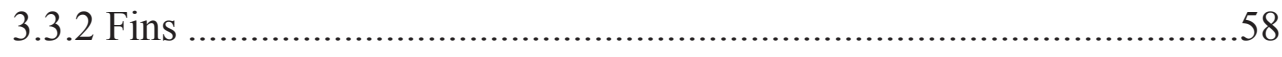

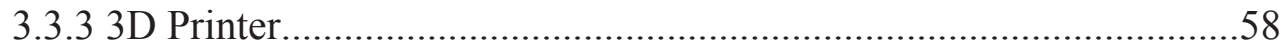

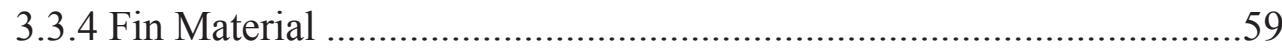

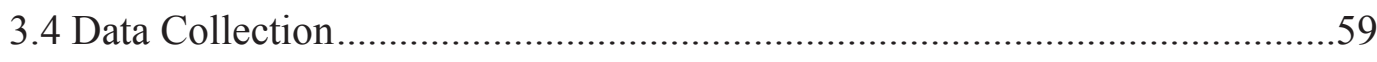

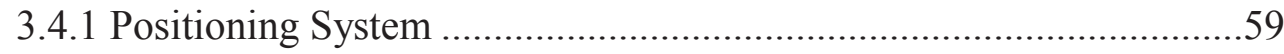

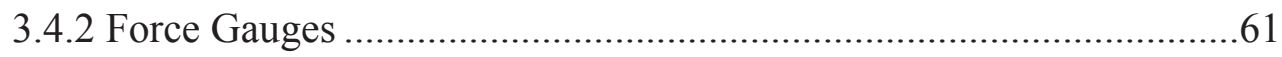

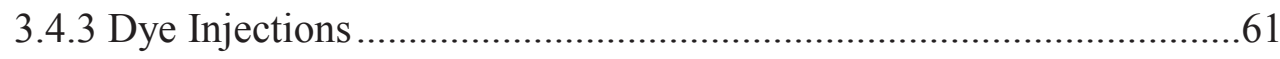




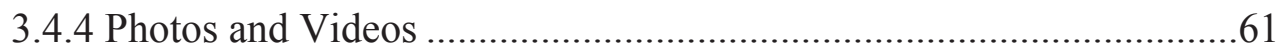

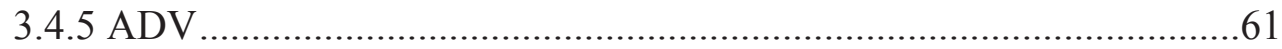

Chapter 4: CFD for Single Fin .......................................................................................63

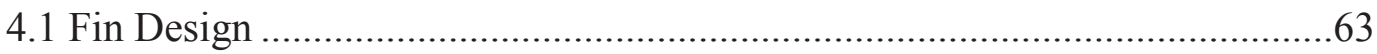

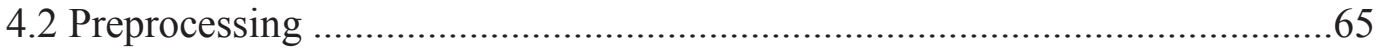

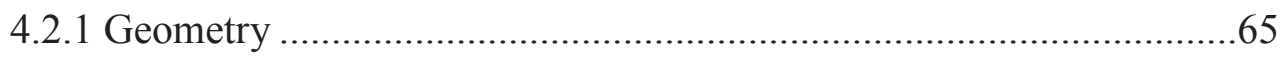

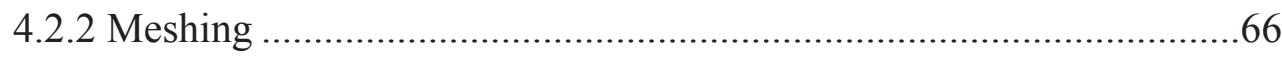

4.2.3 Modeling Method ............................................................................67

4.2.4 Boundary Conditions ........................................................................67

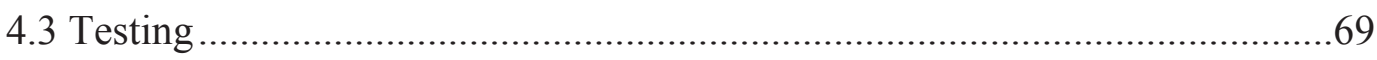

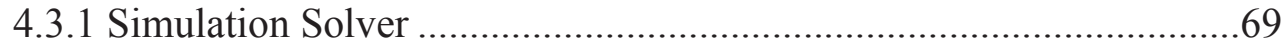

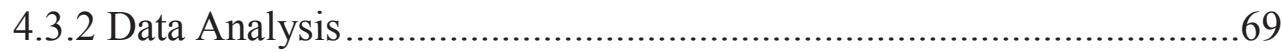

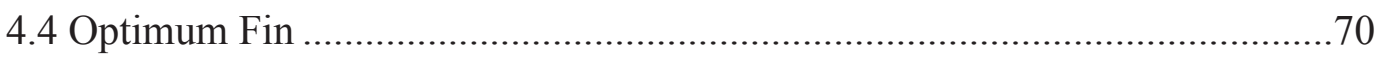

4.4.1 Preprocessing ..................................................................................70

4.4.2 Data Analysis..................................................................................

4.4.3 Visual Flow Analysis.......................................................................

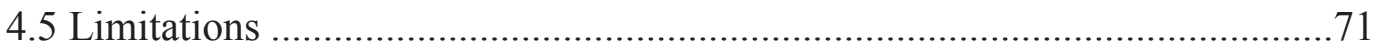

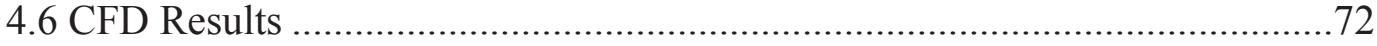

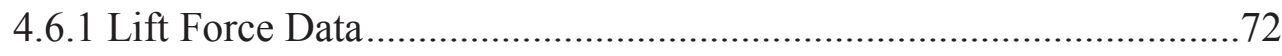

4.6.2 Drag Force Data...............................................................................74

4.4.3 Lift-to-Drag Ratios .........................................................................

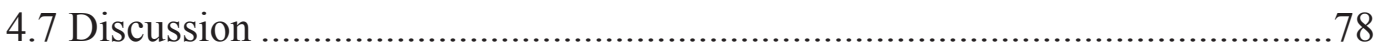

Chapter 5: Laboratory Work for Single Fin ...............................................................81

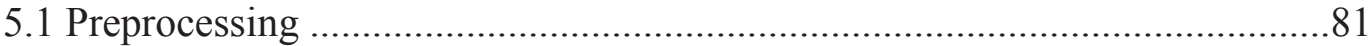

5.1.1 Scaling Surfboard and Fins................................................................ 81

5.1.2 Fin Printing and Fabrication ............................................................ 81

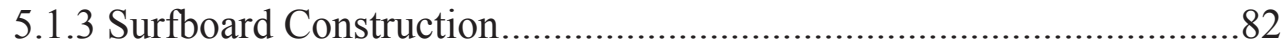

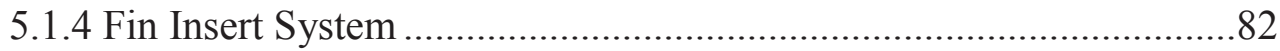

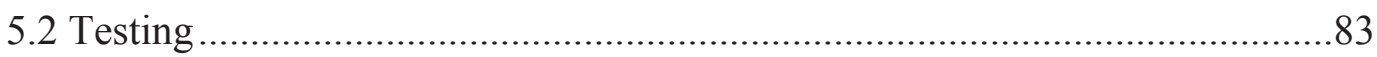




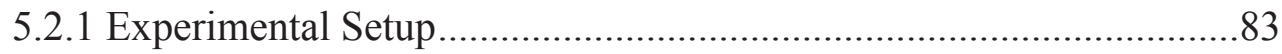

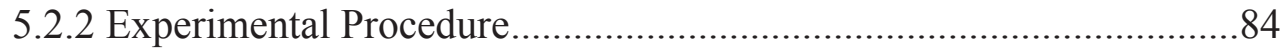

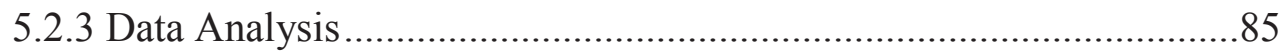

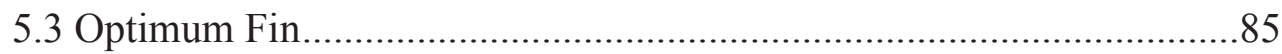

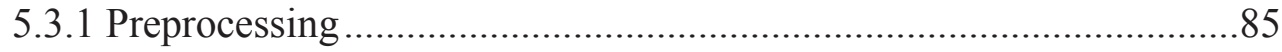

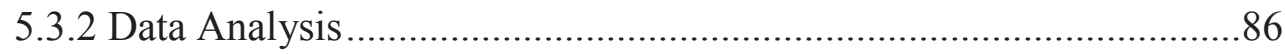

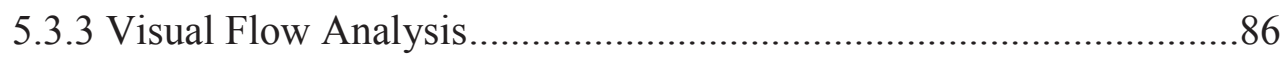

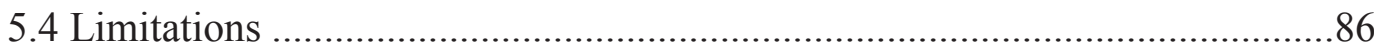

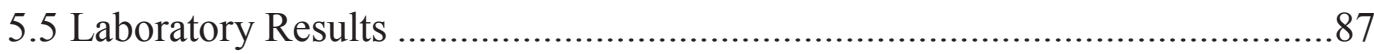

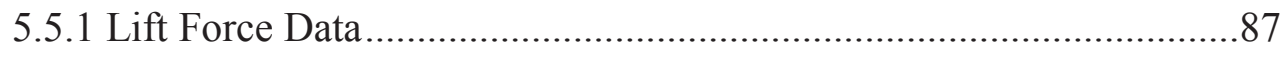

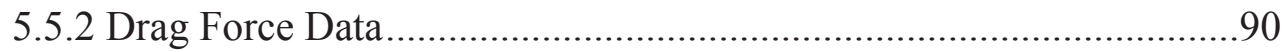

5.5.3 Lift-to-Drag Ratios .........................................................................91

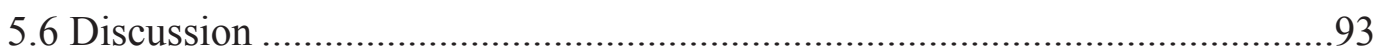

Chapter 6: Optimum Fin..................................................................................................99

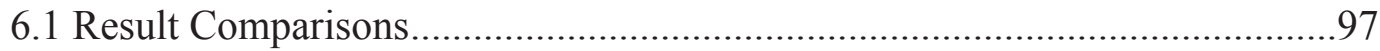

6.1.1 CFD Lift Coefficients ....................................................................97

6.1.2 Lab Lift Coefficients..........................................................................99

6.1.3 CFD Drag Coefficients .....................................................................101

6.1.4 Lab Drag Coefficients...................................................................103

6.1.5 Lift-to-Drag Ratios ......................................................................105

6.2 Optimum Fin Results ...........................................................................106

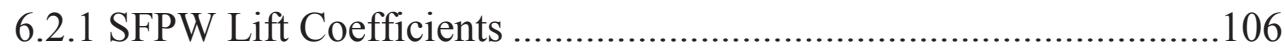

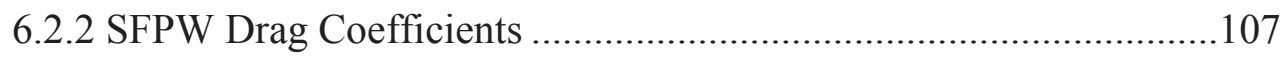

6.2.3 High Velocity Analysis.....................................................................108

6.2.4 CFD Visual Analysis .......................................................................113

6.2.5 Lab Visual Analysis.......................................................................135

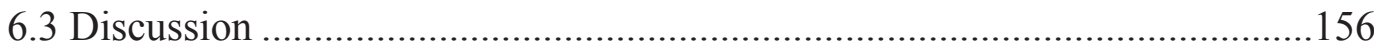

6.3.1 Result Comparisons …………………………………..................156

6.3.2 Optimum Fin Discussion .................................................................157

6.3.3 High Velocity Discussion ..............................................................158 


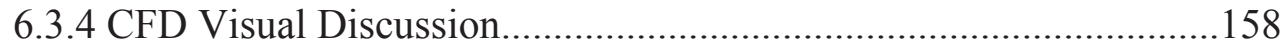

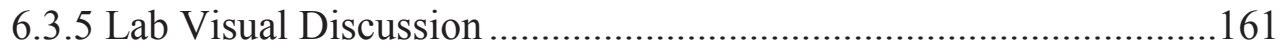

Chapter 7: Future Work ...........................................................................167

Chapter 8: Summary and Conclusion..............................................................169

Chapter 9: References ............................................................................................173

Appendix A: Methods for Experimental Setup...............................................178

Appendix B: Results for Remaining Nine Fins .................................................186 


\section{List of Figures}

Figure 1: Basic design features of a surfboard fin (Image by author, 2015) ...........28

Figure 2: Hydrodynamics terms of a hydrofoil (Image by author, 2015) after (U.S.

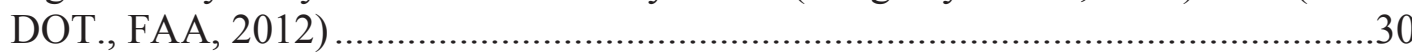

Figure 3: Basic design features of a surfboard (Image by author, 2015).................31

Figure 4: Velocity gradient from boundary layer effects (Image by author, 2015) after (Anderson, 2005)

Figure 5: View of the water channel and experimental setup from the downstream (outlet) section. List below indicates feature details. (Image by author, 2015)

Figure 6: View of the water channel and experimental setup from the upstream (inlet) section. List below indicates feature details. (Image by author, 2015)..........56

Figure 7: Plan view of the pump and water channel (outlet section) setup. List below indicates feature details. (Image by author, 2015).

Figure 8: Experimental section (A) Plan view of surfboard and spring gaugemeasuring lift force (B) Plan view of surfboard and spring gauge-measuring drag force (C) isometric view of the positioning system (Image by author, 2015)...60

Figure 9: Spring gauges used for the lab lift and drag force (Image by author, 2015) .61

Figure 10: Spotted Dolphin spline and foil shapes (Image by author, 2015) ..........63

Figure 11: Meshing of a Blue Shark fin (Image by author, 2015) .........................66

Figure 12: Boundary conditions around a Blue Shark fin at $0^{\circ}$ angle of attack (Image by author, 2015)

Figure 13: Lift (N) from CFD data collected for nine fins at ten angles of attack $\left(0-45^{\circ}\right), \mathrm{U} \infty=0.5 \mathrm{mps}$

Figure 14: Drag $(\mathrm{N})$ from CFD data collected for nine fins at ten angles of attack $\left(0-45^{\circ}\right), \mathrm{U}_{\infty}=0.5 \mathrm{mps}$.

Figure 15: Lift to drag ratio values from CFD data collected for nine fins at ten angles of attack $\left(0-45^{\circ}\right), \mathrm{U} \infty=0.5 \mathrm{mps}$

Figure 16: Lift $(\mathrm{N})$ values from experimental data collected for nine fins at ten angles of attack $\left(0-45^{\circ}\right), U_{\infty}=0.5 \mathrm{mps}$ 
Figure 17: Drag $(\mathrm{N})$ values from experimental data collected for nine fins at ten angles of attack $\left(0-45^{\circ}\right), \mathrm{U} \infty=0.5 \mathrm{mps}$

Figure 18: Lift to drag ratio values from experimental data collected for nine fins at ten angles of attack $\left(0-45^{\circ}\right), \mathrm{U} \infty=0.5 \mathrm{mps}$ .92

Figure 19: Lift coefficients computed from CFD data collected for nine fins at ten angles of attack $\left(0-45^{\circ}\right), \mathrm{U} \infty=0.5 \mathrm{mps}$

Figure 20: Lift coefficients computed from lab data collected for nine fins at ten angles of attack $\left(0-45^{\circ}\right), \mathrm{U} \infty=0.5 \mathrm{mps}$

Figure 21: Drag coefficients computed from CFD data collected for nine fins at ten angles of attack $\left(0-45^{\circ}\right), \mathrm{U} \infty=0.5 \mathrm{mps}$

Figure 22: Drag coefficients computed from lab data collected for nine fins at ten angles of attack $\left(0-45^{\circ}\right), \mathrm{U} \infty=0.5 \mathrm{mps}$

Figure 23: Coefficient of lift from experimental and CFD data collected for the SFPW at ten angles of attack $\left(0-45^{\circ}\right), \mathrm{U} \infty=0.5 \mathrm{mps}$

Figure 24: Coefficient of drag from experimental and CFD data collected for the SFPW at ten angles of attack $\left(0-45^{\circ}\right), \mathrm{U} \infty=0.5 \mathrm{mps}$

Figure 25: Lift (N) from CFD data collected for the SFPW at six angles of attack $\left(0-25^{\circ}\right)$ at three typical surfing velocities (Uळ=3mps, 5mps, and $\left.7 \mathrm{mps}\right) \ldots 109$

Figure 26: Drag (N) from CFD data collected for the SFPW at six angles of attack $\left(0-25^{\circ}\right)$ at three typical surfing velocities (Uळ=3mps, 5mps, and $\left.7 \mathrm{mps}\right) \ldots . .109$

Figure 27: L/D ratio from CFD data collected for the SFPW at six angles of attack $\left(0-25^{\circ}\right)$ at three typical surfing velocities $(\mathrm{U} \infty=3 \mathrm{mps}, 5 \mathrm{mps}$, and $7 \mathrm{mps}) \ldots .112$

Figure 28: Plan view at the base section of the SFPW at $\alpha=0^{\circ}, \mathrm{U} \infty=0.5 \mathrm{mps}$

(A) Velocity magnitudes. (B) Velocity magnitudes and flow vectors

(C) Streamlines

Figure 29: Isolines displaying static pressure distribution $\left(\mathrm{N} / \mathrm{m}^{2}\right)$ of the plan view at the base section of the SFPW at $\alpha=0^{\circ}, \mathrm{U} \infty=0.5 \mathrm{mps}$

Figure 30: SFPW at $\alpha=0^{\circ}, \mathrm{U} \infty=0.5 \mathrm{mps}$ (A) Velocity magnitudes and flow vectors, plan view at the mid-section (B) Streamlines, isometric view from trailing edge of the fin.....

Figure 31: Profile view at the leading-edge section of the SFPW at $\alpha=0^{\circ}$, Uo $=0.5 \mathrm{mps}(A)$ Velocity magnitudes (B) Flow vectors

Figure 32: Profile view at the upper camber surface section of the SFPW at $\alpha=0^{\circ}, \mathrm{U} \infty=0.5 \mathrm{mps}(A)$ Flow vectors $(B)$ Velocity magnitudes .... 
Figure 33: Plan view at the base section of the SFPW at $\alpha=10^{\circ}, \mathrm{U} \infty=0.5 \mathrm{mps}$

(A) Velocity magnitudes. (B) Velocity magnitudes and flow vectors

(C) Streamlines

Figure 34: Isolines displaying static pressure distribution $\left(\mathrm{N} / \mathrm{m}^{2}\right)$ of the plan view at the base section of the SFPW at $\alpha=10^{\circ}, \mathrm{U} \infty=0.5 \mathrm{mps}$

Figure 35: SFPW at $\alpha=10^{\circ}, \mathrm{U} \infty=0.5 \mathrm{mps}(A)$ Velocity magnitudes and flow vectors, plan view at the mid-section (B) Streamlines, isometric view from trailing edge of the fin....

Figure 36: Profile view at the leading-edge section of the SFPW at $\alpha=10^{\circ}$, U $\infty=0.5 \mathrm{mps}(A)$ Flow vectors $(B)$ Velocity magnitudes

Figure 37: Profile view at the mid-edge section of the SFPW at $\alpha=10^{\circ}$, $\mathrm{U} \infty=0.5 \mathrm{mps}$ (A) Flow vectors (B) Velocity magnitudes

Figure 38: Profile view at the trailing-edge section of the SFPW at $\alpha=10^{\circ}$, Uo $=0.5 \mathrm{mps}(A)$ Flow vectors $(B)$ Velocity magnitudes

Figure 39: Plan view at the base section of the SFPW at $\alpha=25^{\circ}, \mathrm{U} \infty=0.5 \mathrm{mps}$

(A) Velocity magnitudes. (B) Velocity magnitudes and flow vectors.

(C) Streamlines

Figure 40: Isolines displaying static pressure distribution $\left(\mathrm{N} / \mathrm{m}^{2}\right)$ of the plan view at the base section of the SFPW at $\alpha=25^{\circ}, \mathrm{U} \infty=0.5 \mathrm{mps}$

Figure 41: SFPW at $\alpha=25^{\circ}, \mathrm{U} \infty=0.5 \mathrm{mps}(A)$ Velocity magnitudes and flow vectors, plan view at the mid-section (B) Streamlines, isometric view from trailing edge of the fin....

Figure 42: Profile view at the leading-edge section of the SFPW at $\alpha=25^{\circ}$, $\mathrm{U} \infty=0.5 \mathrm{mps}(A)$ Flow vectors $(B)$ Velocity magnitudes

Figure 43: Profile view at the mid-edge section of the SFPW at $\alpha=25^{\circ}$, $\mathrm{U} \infty=0.5 \mathrm{mps}(A)$ Flow vectors $(B)$ Velocity magnitudes

Figure 44: Profile view at the trailing-edge section of the SFPW at $\alpha=25^{\circ}$, $\mathrm{U} \infty=0.5 \mathrm{mps}(A)$ Flow vectors $(B)$ Velocity magnitudes

Figure 45: Plan view at the base section of the SFPW at $\alpha=45^{\circ}, \mathrm{U} \infty=0.5 \mathrm{mps}$

(A) Velocity magnitudes. (B) Velocity magnitudes and flow vectors.

(C) Streamlines

Figure 46: Isolines displaying static pressure distribution $\left(\mathrm{N} / \mathrm{m}^{2}\right)$ of the plan view at the base section of the SFPW at $\alpha=45^{\circ}, \mathrm{U}_{\infty}=0.5 \mathrm{mps}$ 
Figure 47: SFPW at $\alpha=45^{\circ}, \mathrm{U} \infty=0.5 \mathrm{mps}$ (A) Velocity magnitudes and flow vectors, plan view at the mid-section (B) Streamlines, isometric view from trailing edge of the fin.

Figure 48: Profile view at the leading-edge section of the SFPW at $\alpha=45^{\circ}$, $\mathrm{U} \infty=0.5 \mathrm{mps}(A)$ Flow vectors $(B)$ Velocity magnitudes

Figure 49: Profile view at the mid-edge section of the SFPW at $\alpha=45^{\circ}$, $\mathrm{U} \infty=0.5 \mathrm{mps}(A)$ Flow vectors $(B)$ Velocity magnitudes

Figure 50: Profile view at the trailing-edge section of the SFPW at $\alpha=45^{\circ}$, $\mathrm{U} \infty=0.5 \mathrm{mps}(A)$ Flow vectors $(B)$ Velocity magnitudes

Figure 51: Plan view of the flow past the SFPW at $\alpha=0^{\circ}, \mathrm{U}=0.5 \mathrm{mps}$ using dye injections at the base section

Figure 52: Plan view of the flow past the SFPW at $\alpha=0^{\circ}, U_{\infty}=0.5 \mathrm{mps}$ using dye injections at the mid-section.

Figure 53: Profile view of the flow past the SFPW at $\alpha=0^{\circ}, U_{\infty}=0.5 \mathrm{mps}$ using dye injections at the base section

Figure 54: Profile view of the flow past the SFPW at $\alpha=0^{\circ}, U_{\infty}=0.5 \mathrm{mps}$ using dye injections at the mid-section....

Figure 55: Profile view of the flow past the SFPW at $\alpha=0^{\circ}, U_{\infty}=0.5 \mathrm{mps}$ using dye injections at the fin tip....

Figure 56: Plan view of the flow past the SFPW at $\alpha=10^{\circ}, U_{\infty}=0.5 \mathrm{mps}$ using dye injections at the base section (A) compression side. Sequence from left to right (B) suction side. Sequence from left to right.

Figure 57: Profile view of the flow past the SFPW at $\alpha=10^{\circ}, U_{\infty}=0.5 \mathrm{mps}$ using dye injections on the compression side at the base section. Sequence from left to right.

Figure 58: Profile view of the flow past the SFPW at $\alpha=10^{\circ}, U_{\infty}=0.5 \mathrm{mps}$ using dye injections on the suction side at the base section. Sequence from left to right.

Figure 59: Plan view of the flow past the SFPW at $\alpha=10^{\circ}, U_{\infty}=0.5 \mathrm{mps}$ using dye injections at the mid-section (A) compression side. Sequence from left to right (B) suction side. Sequence from left to right.

Figure 60: Profile view of the flow past the SFPW at $\alpha=10^{\circ}, U_{\infty}=0.5 \mathrm{mps}$ using dye injections on the compression side at the mid-section. Sequence from left to right. 
Figure 61: Profile view of the flow past the SFPW at $\alpha=10^{\circ}, U_{\infty}=0.5 \mathrm{mps}$ using dye injections on the suction side at the mid-section. Sequence from left to right.....

Figure 62: Profile view of the flow past the SFPW at $\alpha=10^{\circ}, U_{\infty}=0.5 \mathrm{mps}$ using dye injections at the fin tip

Figure 63: Plan view of the flow past the SFPW at $\alpha=25^{\circ}, U_{\infty}=0.5 \mathrm{mps}$ using dye injections at the base section (A) compression side. Sequence from left to right (B) suction side. Sequence from left to right.

Figure 64: Profile view of the SFPW at $\alpha=25^{\circ}, U_{\infty}=0.5 \mathrm{mps}$ using dye injections on the compression side at the base section. Sequence from left to right.

Figure 65: Profile view of the SFPW at $\alpha=25^{\circ}, U_{\infty}=0.5 \mathrm{mps}$ using dye injections on the suction side at the base section. Sequence from left to right

Figure 66: Plan view of the SFPW at $\alpha=25^{\circ}, U_{\infty}=0.5 \mathrm{mps}$ using dye injections at the mid-section $(A)$ compression side. Sequence from left to right (B) suction side. Sequence from left to right

Figure 67: Profile view of the SFPW at $\alpha=25^{\circ}, U_{\infty}=0.5 \mathrm{mps}$ using dye injections on the compression side at the mid-section. Sequence from left to right.

Figure 68: Profile view of the SFPW at $\alpha=25^{\circ}, U_{\infty}=0.5 \mathrm{mps}$ using dye injections on the suction side at the mid-section. Sequence from left to right.

Figure 69: Profile view of the SFPW at $\alpha=25^{\circ}, U_{\infty}=0.5 \mathrm{mps}$ using dye injections at the fin tip.

Figure 70: Plan view of the SFPW at $\alpha=45^{\circ}, U_{\infty}=0.5 \mathrm{mps}$ using dye injections at the base section $(A)$ compression side. Sequence from left to right (B) suction side. Sequence from left to right.

Figure 71: Profile view of the SFPW at $\alpha=45^{\circ}, U_{\infty}=0.5 \mathrm{mps}$ using dye injections on the compression side at the base section. Sequence from left to right.

Figure 72: Profile view of the SFPW at $\alpha=45^{\circ}, U_{\infty}=0.5 \mathrm{mps}$ using dye injections on the suction side at the base section. Sequence from left to right

Figure 73: Plan view of the SFPW at $\alpha=45^{\circ}, U_{\infty}=0.5 \mathrm{mps}$ using dye injections at the mid-section (A) compression side. Sequence from left to right (B) suction side. Sequence from left to right 
Figure 74: Profile view of the SFPW at $\alpha=45^{\circ}, U_{\infty}=0.5 \mathrm{mps}$ using dye injections on the compression side at the mid-section. Sequence from left to right.

Figure 75: Profile view of the SFPW at $\alpha=45^{\circ}, U_{\infty}=0.5 \mathrm{mps}$ using dye injections on the suction side at the mid-section. Sequence from left to right

Figure 76: Profile view of the SFPW at $\alpha=45^{\circ}, U_{\infty}=0.5 \mathrm{mps}$ using dye injections at the fin tip 


\section{List of Tables}

Table 1: Dimensions of original, flume-scaled, and full-scaled fins for nine fin types 65

Table 2: Ambient Conditions of Water (Kundu \& Cohen, 2008)...........................68

Table 3: Species name and acronym code ....................................................... 72

Table 4: Lift (N) from CFD data collected for nine fins at ten angles of attack $\left(0-45^{\circ}\right), \mathrm{U} \infty=0.5 \mathrm{mps}$

Table 5: Drag (N) from CFD data collected for nine fins at ten angles of attack $\left(0-45^{\circ}\right), \mathrm{U} \infty=0.5 \mathrm{mps}$

Table 6: Lift to Drag Ratio (L/D) from CFD data collected for nine fins at ten angles of attack $\left(0-45^{\circ}\right), \mathrm{U} \infty=0.5 \mathrm{mps}$

Table 7: Lift $(\mathrm{N})$ values from experimental data collected for nine fins at ten

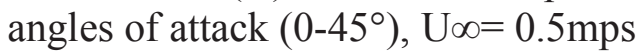

Table 8: Drag $(\mathrm{N})$ values from experimental data collected for nine fins at ten angles of attack $\left(0-45^{\circ}\right)$, Uळ $=0.5 \mathrm{mps}$

Table 9: Lift to Drag ratio (L/D) from experimental data collected for nine fins at ten angles of attack $\left(0-45^{\circ}\right), \mathrm{U} \infty=0.5 \mathrm{mps}$

Table 10: Lift coefficients computed from CFD data collected for nine fins at ten angles of attack $\left(0-45^{\circ}\right), \mathrm{U} \infty=0.5 \mathrm{mps}$..........

Table 11: Lift coefficients computed from lab data collected for nine fins at ten angles of attack $\left(0-45^{\circ}\right)$, Uo $=0.5 \mathrm{mps}$

Table 12: Drag coefficients computed from CFD data collected for nine fins at ten angles of attack $\left(0-45^{\circ}\right), \mathrm{U} \infty=0.5 \mathrm{mps}$

Table 13: Drag coefficients computed from lab data collected for nine fins at ten angles of attack $\left(0-45^{\circ}\right), \mathrm{U} \infty=0.5 \mathrm{mps}$

Table 14: Lift $(\mathrm{N})$ and drag $(\mathrm{N})$ forces from CFD data collected for the SFPW at six angles of attack $\left(0-25^{\circ}\right)$ at three typical surfing velocities (Uळ=3mps, $5 \mathrm{mps}$, and $7 \mathrm{mps}$ ) 


\section{Nomenclature}

\begin{tabular}{|c|c|}
\hline $\operatorname{Re}$ & $=$ Reynolds number \\
\hline$C_{L}$ & $=$ Lift Coefficient \\
\hline$C_{D}$ & $=$ Drag Coefficient \\
\hline$A_{w}$ & $=$ Wing Area $\left(\mathrm{m}^{2}\right)$ \\
\hline$A$ & $=$ Reference Area $\left(\mathrm{m}^{2}\right)$ \\
\hline$A_{S}$ & $=$ Surface Area $\left(\mathrm{m}^{2}\right)$ \\
\hline $\mathrm{N}$ & $=$ Newtons \\
\hline$F_{D}$ & $=$ Drag Force $(\mathrm{N})$ \\
\hline$F_{L}$ & $=$ Lift Force $(\mathrm{N})$ \\
\hline$g$ & $=$ Gravitation Force $\left(\mathrm{m} / \mathrm{s}^{2}\right)$ \\
\hline$L$ & $=$ Length $(\mathrm{m})$ \\
\hline$L_{c}$ & $=$ Chord Length (m) \\
\hline$L_{w}$ & $=$ Wingspan Length $(\mathrm{m})$ \\
\hline$V$ & $=$ Volume $\left(\mathrm{m}^{3}\right)$ \\
\hline$U_{\infty}$ & $=$ Free-stream Velocity $(\mathrm{m} / \mathrm{s})$ \\
\hline$T$ & $=$ Temperature $\left({ }^{\circ} \mathrm{C}\right)$ \\
\hline$P$ & $=$ Absolute Pressure $(\mathrm{MPa})$ \\
\hline$P_{\infty}$ & $=$ Free-stream Hydrostatic Pressure \\
\hline$P_{t}$ & $=$ Total Pressure \\
\hline$P_{S}$ & $=$ Static Pressure \\
\hline$\left(\rho U^{2}\right) / 2$ & $=$ Dynamic Pressure \\
\hline$\mu$ & $=$ Shear Viscosity $(\mathrm{kg} / \mathrm{m} \cdot \mathrm{s})$ \\
\hline$\rho$ & $=$ Density $\left(\mathrm{kg} / \mathrm{m}^{3}\right)$ \\
\hline$\rho_{\infty}$ & $=$ Free-stream Density $\left(\mathrm{kg} / \mathrm{m}^{3}\right)$ \\
\hline$\tau_{\mathrm{w}}$ & $=$ Shear Stress $\left(\mathrm{N} / \mathrm{m}^{2}\right)$ \\
\hline$\alpha$ & $=$ Angle of Attack $\left(^{\circ}\right)$ \\
\hline$k$ & $=$ Turbulence kinectic energy $\left(\mathrm{m}^{2} / \mathrm{s}^{2}\right)$ \\
\hline$\varepsilon$ & $=$ Turbulence dissipation $\left(\mathrm{m}^{2} / \mathrm{s}^{3}\right)$ \\
\hline$C_{\mu}$ & $=k-\varepsilon$ model coefficient $(0.09)$ \\
\hline$\mu_{T}$ & $=$ Eddy Viscosity $(\mathrm{kg} / \mathrm{m} \cdot \mathrm{s})$ \\
\hline$I$ & $=$ Turbulence Intensity $(\%)$ \\
\hline
\end{tabular}




\section{Acknowledgements}

Dr Barkdoll

Thomas David Foster Yeoman

Mom, Dad and Scott (as well as the support from my friends and family)

Jennie Tyrell

Julia Rice

Greg Houle and Brent Centraco 



\begin{abstract}
Wave surfing is a multi-billion dollar industry involving both maneuverability and speed, yet little research has been done regarding optimal fins shape for these competing qualities. The purpose of this master's thesis was to focus on a single fin setup in order to identify a bio-inspired fin shape that maximized lateral stability while minimizing drag forces, in order to increase surfing maneuverability.

The computational fluid dynamic models NX and laboratory experiments performed in a water channel, with lift and drag being directly measured, were used to compare nine fins based on dorsal fins of real fish. To properly compare, fluid conditions were comparable between the CFD and lab experiments. It was found that the Short-finned Pilot Whale at an angle of attack of $10^{\circ}$ had the greatest lift-to-drag ratios. Flow patterns around fins at a low angle of attack were smooth with negligible flow separation, while at any angle of attack greater than $25^{\circ}$, flow separation-induced drag forces became excessive.
\end{abstract}





\section{Chapter 1: Introduction and Motivation}

\section{$1.1 \quad$ INTRODUCTION}

As one of the most popular sports worldwide, surfing is a global industry that generates billions of dollars annually. Surfing is one of the oldest known sports still practiced today. Beginning around three thousand years ago, small fishing boats surfed the waves into shore to safely bring their communities food. Overtime, this evolved into a pastime for amusement and the boats were replaced with long wooden surfboards. It was not until the 1930's that the first fin was introduced and the entire sport of surfing was forever changed. Today, both professional and amateur riders surf on ocean, lake, river, and man-made waves. Many fin sizes, shapes, materials, and setups are on the current market for specific wave types, as well as riders of different weights, surfing styles and preferences. However, there are few scientifically published articles that present quantitative data to endorse the different fin parameters for specific applications.

Traditionally, shapers and professional surfers work together to improve the designs of surfboards and fins, but there are problems with this method. It is common for several variables to simultaneously change during the shaping-testing process, so the specific adjustment to the improvement of the setup might be unknown. Each surfer also has a different preference and style, which leads to inconsistencies in the design feedback. Different aspects of the surfboard and fin setup that are favorable to a professional might not be practical for a novice rider. Hendricks (1969) has determined that speed, maneuverability, and control are major considerations in the design during the shaping process. The driving forces are lift and drag, relating to the fluid properties as well as the objects shape, size, and wetted perimeter characteristics. Carswell and Lavery (2006) thought that the profile shape of a fin might have a dramatic effect on the performance and this parameter should be studied in future work.

Many surfboard fin shapes have derived from the fin features of aquatic mammals and fish (Sakamoto \& Yanamoto, 2007). The emulation of biological characteristics can improve the design and performance of a surfboard fin (Fish, et al., 2011). For this thesis, nine bio- 
inspired fins were produced for comparisons in order to result in an optimum fin that generated high lift and low drag forces. By specifying constant variables for base lengths, fin heights, and hydrofoil shapes, the varying profile shapes could be examined and compared. Lift and drag forces were obtained in computational and laboratory experiments. The lift and drag coefficients were calculated to evaluate the results in these two data gathering methods. One optimum fin was defined for further high velocity measurements and flow field analysis.

There have only been a few scientific studies on surfboard fin design using computational or experimental analysis of lift and drag force results. There are no known studies presenting the evaluations of fin profile dimensions based on bio-inspired dorsal fin shapes, so result comparisons to other studies could not be made. The goal of this thesis is to provide a basis for further research on the hydrodynamic effects based on fin geometry and the design of surfboard fins.

\subsection{OBJECTIVES}

The objective of this thesis was to find an optimal single fin shape at an ideal angle of attack to optimize maneuverability and speed.

\subsection{BACKGROUND}

\subsubsection{WHAT IS WAVE SURFING}

Wave surfing is considered an extreme sport and a leisurely pastime. It is an action in which the riders use a breaking water wave to propel themselves towards shore (Paine, 1974). A surfer can be defined as anyone who rides a wave, whether it be standing on a surfboard or bodysurfing. Surfers can range in experience from beginners to professional athletes.

Most surfers use a buoyant surfboard in order to catch, ride, and maneuver on breaking waves. A fin is a complex hydrofoil skeg, mounted on the bottom of a surfboard. A fin or sets of fins are generally attached to the tail of the surfboard. This provides the rider with directional stability and control by using their feet to steer. The rider can shift their weight in order to change direction and speed. 


\subsubsection{WAVE THEORY}

There are many types of waves produced on large bodies of water. Typical surf waves are wind driven from distant storm systems (Butt, 2008). Generally, these low-pressure systems will blow across an area, known as a fetch, for an extended period of time (Paine, 1974). This disturbing force causes small ripples on the surface of the water called capillary waves (Beggs, 2009). The surface tension of the water is the restoring force (Butt, 2008). Strong, continuous wind can cause capillary waves to transition into gravitational waves. Over time, capillary waves begin to accrue and once wave heights reach beyond $0.02 \mathrm{~m}$ they become gravitational waves (Beggs, 2009). Gravitational waves can continue to build in size as multiple waves merge together within the fetch (Beggs, 2009). The wave height and length produced within the fetch are a function of wind speed and fetch length (Butt, 2008). Once the waves leave the fetch, they can travel long distances before they reach shore (Beggs, 2009). As waves generate in the direction of the wind and approach the coast, they will move from deep-water to shallow-water waves (Paine, 1974).

To be considered a deep-water wave, the wavelength must be less than the depth of the water (Paine, 1974). Water movement is negligible below the wave base depth (Beggs, 2009). The deep-water waves move sinusoidally which propagates a circular orbit motion of the wave particles. The rotating shape diameter diminishes exponentially with depth (Edge, 2001). The particles move forward at the crest and backward at the trough as they rotate, causing the wave to have no net motion (Edge, 2001). This forms a trochoidal profile shape, causing the wave crests to be sharper than the troughs (Paine, 1974). The speeds of the wave particles are much slower than that of the celerity (wave velocity) (Beggs, 2009).

The celerity of shallow-water waves is dependent on wave height and water depth (Paine, 1974). So as water depth decreases in height, wave velocity begins to slow and wave amplitude increases (Beggs, 2009). Consequently, the wave height increases until it becomes too unstable and breaks (Paine, 1974). The water particle movement can be explained by the change in wave height. The slope of the floor in the ocean or lake affects the shape of the water particles flow path in shallow-water regions (Beggs, 2009). As the wave travels into shallow water, the particles start to orbit from the small circular shape 
into large elliptical paths (Paine, 1974). As the path becomes more elliptical, the particle speeds increase (Beggs, 2009). Once the velocity of the water particles and wave celerity are equivalent, the wave breaks (Beggs, 2009). The point at which the wave starts to break is the area in which the surfer would ride the wave.

Surfing velocities are dependent on wave height and length, wave velocity, position on the wave, surfing ability, surfboard and fin drag, and surfers weight (Hendricks, Surfboard Hydrodynamics, Part IV: Speed, 1969) (Edge, 2001). For a typical surfing wave height ( $\sim .5$ to $2.5 \mathrm{~m}$ ) velocities can reach up to $7 \mathrm{mps}$ (Hendricks, Surfboard Hydrodynamics, Part IV: Speed, 1969) (Edge, 2001). The speed of the surfer can exceed the waves speed though. For a $1.5 \mathrm{~m}$ wave with a velocity of $3.8 \mathrm{mps}$, the surfer can reach speeds of more than $6 \mathrm{mps}$ (Lavery, Foster, Carswell, \& Brown, 2009). Surfers are also known to ride larger waves. A double overhead wave is a wave in which the height is two times the height of the surfer; this is a medium to large wave. A triple overhead wave is considered big-wave surfing, in which case the rider needs to be towed-in by a watercraft vehicle. One of the largest waves ever surfed was 23.8m, by Garrett McNamara (Guinness World Records News, 2012). In these cases, the surfers' velocities are unknown.

\subsubsection{HISTORY}

Today's surfboard and fin designs are the products of several hundred years of trial and error. The relationships between the surfboard shaper and the surfer have created an evolution of both material and design. The first evidence of surfing, dating back to 3000 $\mathrm{BC}$, was discovered on the shores of Peru. Fishing boats, called Tups, were used to easily paddle through waves breaks. From around 2000 BC to 400 AC, western Polynesians began migrating to the Hawaiian Islands. It was here that the practice of wave riding developed into a sport of enjoyment. Replaced by Tups, were four to five meter long hardwood boards. As technology improved over time, so did the materials and shapes of surfboards. (Surfing Heritage Foundation, N/A)

Before the invention of fins, Hawaiians used their feet to maneuver (Surfing Heritage Foundation, N/A). It was not until 1935 that the first fin was fixed on a surfboard. Tom Blake attached a single aluminum speedboat keel to the bottom of his surfboard and the 
single fin was invented (Anders, 2014). In 1955 dolphin fins inspired several fin shapes. In 1961 George Greenough invented the high-aspect ratio single fin, which was patterned after a tuna tail. Between the 1960's and 1970's, fin shapes and configurations were experimented with. Through the 1980's to present time, the thruster setup has been the industry standard (George, 2014). Surfers do use other fin systems due to preference and wave-type. This includes the single fin, which is a classic setup for a longboard.

\subsection{PURPOSE OF THE FIN}

\subsubsection{SKEG AND KEEL}

Surfboard fins are similar to a sailboat skeg and keel. The main purposes of these attributes are to provide directional stability and control of the watercraft. The keel of a sailboat is a hydrodynamic element. It is used to counteract the sideways force of wind and convert it into a forward motion. As the boat moves forward, the keel generates lift (Anderson B. , 2008). Surfboard fins counteract the sideways force of the surfer against the forward velocity of the wave. The fins also provide lift to the surfboard and rider so making sharp turns and maneuvering up and down the wave is possible.

\subsubsection{DORSAL FIN}

The dorsal fin is located on the backs of many marine mammals and fish. There can be more than one of these fins. The rudimentary function of a fish or aquatic mammal's dorsal fin is to provide directional stability (Noble, et al., 2011). It keeps the swimming species from rolling while swimming (Standen \& Lauder, 2007). The secondary purpose is to allow quick stopping and turning. The shape of this fin can also help generate thrust (Noble, et al., 2011). A dorsal fin shape and body location is an individual characteristic dependent on species, sex, and individual (Standen \& Lauder, 2007). Another important hydrodynamic function is the angle of attack of the dorsal fin, which is a strong variable in determining vortex characteristics (Standen \& Lauder, 2007).

\subsubsection{FIN HYDRODYNAMICS}

This section gives details on different fin shape variables. The information here is based on the personal surfing experience of the author and common knowledge among surfers 
and shapers, but has not been documented, to the author's knowledge. It is given here to add to the reasoning of the analysis described later.

The shape, material, and size of a fin are important aspects in the design process. By altering one variable, the hydrodynamic effects will change and reflect in the surfer's performance. Figure 1 shows some basic design features that are described below.

The base of a fin is the area in which the fin connects to the surfboard. Its length $\left(\mathrm{L}_{\mathrm{c}}\right)$ is measured from the leading edge to the trailing edge of the foil shape. It is linked to the surfer's ability to accelerate. This means that the greater base length, the faster acceleration the fin can provide to the surfboard for the rider. Fin depth $\left(\mathrm{L}_{\mathrm{w}}\right)$ is the distance the fin penetrates the water. Its length is measured from the base to the tip of the fin. It is believed that a longer fin depth provides more

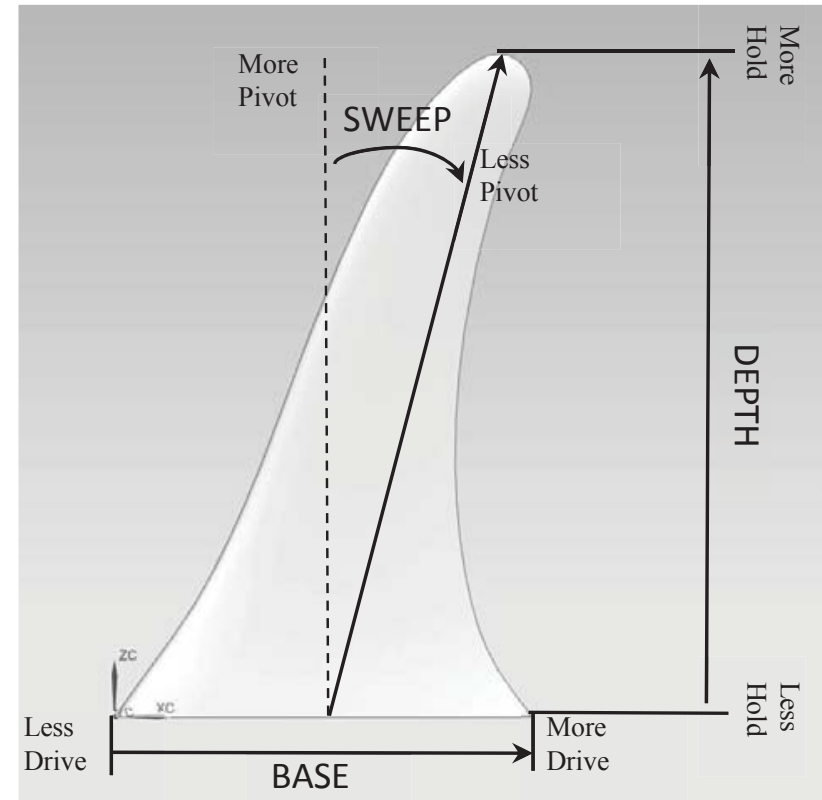

Figure 1: Basic design features of a surfboard fin (Image by author, 2015) hold, which allows the surfer to have more stability. As fin heights are reduced, it could be easier for lateral board sliding to occur. Relating these dimensions to airplane wings, the aspect ratio influences the lift and drag components. Aspect ratio is the proportional relationship of the wing's span length to chord measurement (Anderson, 2005). High aspect ratio wings have a longer span and a smaller chord length. Generally, this provides a higher performance wing with less drag compared to a low aspect ratio wing (U.S. DOT \& FAA, 2012). The three-dimensional fin is made up of two-dimensional hydrofoil shapes across the span of the fin. The fin's shape commonly has a moderate to high aspect ratio. The aspect ratio (AR) differs along the span of the fin. Equation 1 defines the constant length-to-chord ratio:

$$
A R=L_{h}^{2} / A_{w}
$$


where $L_{h}$ is the wing span (length of the fin) and $A_{w}$ is the plan area of the fin. Sweep, also known as rake, is thought to influence the pivoting motion. It is the angle from the midbase point to the tip of the fin. A fin with a lower angled rake means the surfer can make tighter turns. Consequently, this might deliver a fin that is less aerodynamic because the leading edge is more blunt to the oncoming water.

Total surface area of a fin can improve a surfer's stability and possible hold during turns. It does however provide more drag due to a high wetted area. For this reason, the surface of the fin must be completely smooth in order to reduce surface tension and ultimately drag forces. The modern fin can be made from a combination of advanced materials and constructed in various arrangements. Materials can include: glass epoxy, fiberglass, carbon fiber, wood, Texalium, etc. The material design can also provide different levels of distortion. This flex in the fin is dependent on material type and structure. It is the ability of the material to bend, caused by lateral pressure when turning. The stiffer the fin, the more instant response a rider can have to provide speed and drive down a wave.

The placement of the fin on the board is important and is dependent on the rider style, ability, surfboard type, and fin setup. Cant is the angle from the vertical line, perpendicular to the surfboard. The higher the cant angle, the greater the maneuverability a rider will have. Less cant is thought to provide faster acceleration. The toe angle is also a degree setting in relation to the surfboard stringer. These angle settings are only for rail fins. The center fin is always placed symmetrically on the stringer, 90 degree perpendicular to the board.

\subsubsection{NACA AIRFOILS}

An airfoil is a shape, that when passing through a fluid, produces greater lift than drag at an appropriate angle of attack (U.S. DOT \& FAA, 2012). The shape, shown in Figure 2, illustrates the cross-section of an airfoil. For this thesis the foil shape will be passing through water, so it is considered a hydrofoil. The front edge of the foil shape is called the leading edge and it is usually rounded (Kundu \& Cohen, 2008). The rear edge is called the trailing edge and has a sharp pointed shape (U.S. DOT \& FAA, 2012). The distance of the straight line connecting these two points is called the chord (Kundu \& Cohen, 2008). 
Camber is the line midway between the upper and lower sections of the foil. It describes the curvature of the foil, as well as the foils shape. The maximum distance from camber line to the cord line is used as a descriptor

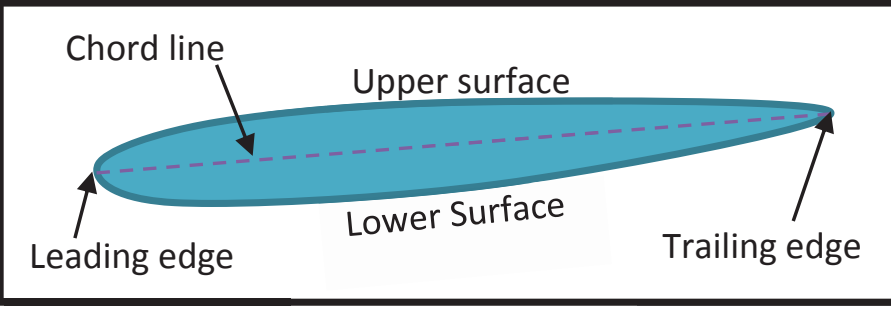

Figure 2: Hydrodynamics terms of a hydrofoil (Image by author, 2015) of the foil shape. This point describes the profile thickness and the thickness distribution. The thickness and distribution are critical mechanisms, which can change acting pressures and velocities (U.S. DOT \& FAA, 2008). The angle from the direction of fluid flow to the chord line expresses the angle of attack. Also known as incident angle, the angle of attack will be discussed further in Section 1.5 (U.S. DOT \& FAA, 2012).

The National Advisory Committee for Aeronautics (NACA) developed a database, which is compiled airfoil shape information. Thousands of airfoil designs were produced and cataloged since the establishment of the NACA in 1915 (Anderson, 2005). Three groups can define the more common shapes: four-digit NACA airfoils, five-digit NACA airfoils, and six-digit NACA airfoils (Sadraey, 2009). The digits describe the airfoil geometry. To give an example, the four-digit series is explained. The first digit gives value to the maximum camber as a percentage of the foil's chord length. The second digit describes the distance from the leading edge to the maximum camber in tenths of percent's of the chord. The last two digits describe the maximum foil thickness as a percentage of the chord length (Hoerner, 1985).

\subsubsection{THE SURFBOARD}

This section gives details on different surfboard shape variables. The information here is based on the personal surfing experience of the author and common knowledge among surfers and shapers, but has not been documented, to the author's knowledge. It is given here to add to the reasoning of the analysis described later. 
Today's surfboard and fin designs are the results sixty years of trial and error. Shapers rely on suggestions from professional surfers to improve the shape (Beggs, 2009). Dimensions of the surfboard are dependent on the surfer's style, ability, weight, and type of wave. Generally, a longboard is used for riders that need more stability and a greater surface area in order to catch and ride the wave. This is because the longboard is more buoyant due to a higher volume compared to a shortboard. Shortboards are designed to allow the rider to maneuver quickly for radical turns. Figure 3 shows a schematic of a surfboard with labeled attributes.

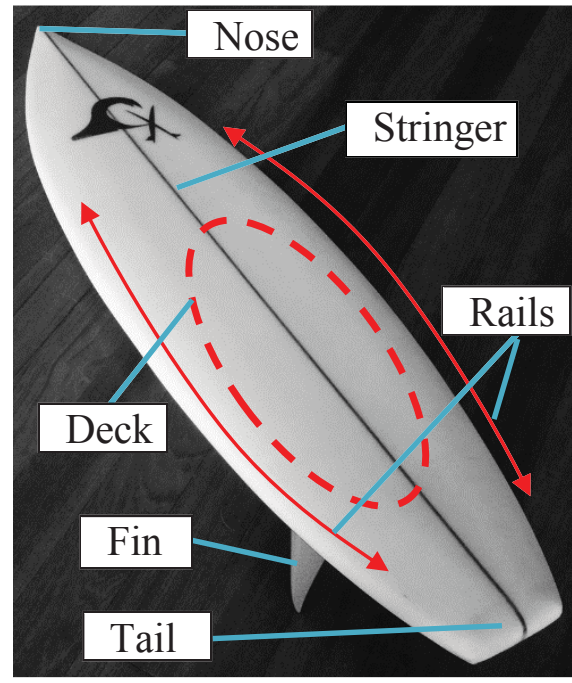

Figure 3: Basic design features of a surfboard (Image by author, 2015)

The rails are the perimeter of the board and consist of two sections: the hard rails and soft rails. The hard rail is a curved edge, which is usually located near the rear of the board. Due to its sharp edge, the hard rails purpose is to separate flow to reduce drag. The more rounded shape of the soft rail, in the mid-section of the board, allows for sharp turning due to increased lift.

The bottom of the board is important because it is the region that the most surface area comes in contact with the water. This section must be very smooth in order to decrease skin friction. It is the planing surface that provides support and lift to the rider and surfboard (Beggs, 2009). The shape of the bottom ranges from flat to a double concave near the tail. Concaves allow the water to channel past the fins and effectively provide increased lift.

At the nose of the board there is a medium rocker. Rocker is the elevated curvature of the board. It has two purposes: to provide stability and a shock absorber. Both the nose and tail shapes vary among surfboards. These shapes provide different hydrodynamic attributes that are thought to improve efficiency, maneuverability, drive, stability, etc. The stringer, which is usually a wood material, is the centerpiece of the board, which provides strength and shape to the surfboard. 


\subsubsection{FIN SETUPS}

This section gives details on different fin setups on a surfboard. The information here is based on the personal surfing experience of the author and common knowledge among surfers and shapers, but has not been documented, to the author's knowledge. It is given here to add to the reasoning of the analysis described later.

Fins are placed on the bottom of the board, near the rear. The fins provide a side force to hold the board from sliding out from under the rider, as well as providing lift for turning purposes. There are many types of fin setups that are appropriate for certain wave types and rider ability, style, size, and preference.

The single fin was the first setup ever placed on a surfboard. In today's application, the single fin is placed on a longboard for smaller waves and long drawn-out turning.

The twin fins, or dual-fin setup, are two fins placed near the rail of the board. As there are only two fins, one acts as a pivoting point for making tight turns. This setup is known for a quick response to maneuvers, but is not as easy to control compared to the thruster.

The thruster, or tri-fin setup, is a three-fin placement on the bottom of the board. Two rail fins and one fin centered on the stringer. This allows the rider to perform tricks and turns with control in a wide range of wave types. The drawback to this setup is the extra fin provides greater drag.

Quads are a four-fin system; two fins near the rails and two trailing fins. These allow the rider stability, hold, and speed on large surf breaks such as Jaws and Mavericks.

There are many other setups including the $2+1$, twinzier, tunnel, bonzer, diamond quad, etc. Each setup has a wide variety of board placement positions, angles of attack, and cant angles. As the setups and positions are modified, the hydrodynamic effects and forces also change.

\subsection{ACTING ForCES}

There are four basic forces that act upon a surfboard as a surfer rides a wave: lift, drag, thrust, and weight. Surfers ride on waves in the ocean and in fresh water, each constituting 
their own physical properties. These fundamental physical quantities are free-stream pressure $P_{\infty}$, density $\rho_{\infty}$, temperature $T$, and velocity $U_{\infty}$ (Anderson, 2005). Components of pressure include velocity, density and shear viscosity $\mu_{\infty}$. Each of these four basic hydrodynamic flow quantities varies depending on the body of water. Lift $\left(\mathrm{F}_{\mathrm{L}}\right)$ and drag $\left(F_{D}\right)$ forces will vary depending on the value of these properties, as presented in Equation 2 and Equation 3. Hence, for a given airfoil shape $A_{w}$ at an identified angle of attack $\alpha$, lift and drag are a function of these values and can be written as:

$$
\begin{array}{ll}
F_{L}=f\left(U_{\infty}, \rho_{\infty}, A_{w}, \mu_{\infty}, \alpha\right) & \text { Equation } 2 \\
F_{D}=f\left(U_{\infty}, \rho_{\infty}, A_{w}, \mu_{\infty}, \alpha\right) & \text { Equation } 3
\end{array}
$$

By understanding the forces and other factors, design improvements can be made to both the surfboard and the fins. For the purpose of this research, the angle of attack relates to the changing direction of the surfboard and single fin setup compared to the oncoming flow (wave flow direction). According to Gudimetla et al. (2009), angles of attack can range from $0^{\circ}$ to $45^{\circ}$.

\subsubsection{LIFT}

Lift occurs when an object in a moving fluid changes the flow direction. As a result, the object will lift in the opposite direction. Both lift and drag are generally dependent on the Reynolds number (Sadraey, 2009). For the purpose of this thesis, lift is a hydrodynamic force generated by the motion of the fin through water. Lift is a vector quantity that has a magnitude and a direction. The airfoil theory states that the direction of lift is through the center of pressure of the fin, perpendicular to the flow direction. Magnitude is dependent on the geometry of the fin setup, the angle of attack, and the fluid motion and characteristics. The lifting action of a surfboard is measured in the positive z-direction. When considering the fin(s), lift is measured in the y-direction. This lifting action provides a side force necessary to hold the surfboard for directional stability, as well as lift to help ease of maneuverability (Brandner \& Walker, 2004).

The fin on a surfboard is a foiled shape, which provides the surfer lateral lift to maneuver and change the direction of the board (Brandner \& Walker, 2004). The surfboard and fin 
together also provide a horizontal lift, but only the lateral lift of a fin was considered for the focus of this thesis. Lift is generated when the fin changes the direction of the flowing fluid causing forces to act perpendicular to the motion of the fluid (Gudimetla, Kelson, \& El-Atm, 2009) (Kundu \& Cohen, 2008). The airstream shape of the fin induces two physical responses to create lift: a positive lifting pressure from below the hydrofoil and a negative lifting pressure from above the hydrofoil (U.S. DOT \& FAA, 2008).

There are two components of total fluid flow pressure: static and dynamic pressure. The total pressure $P_{t}$ is the sum of these two pressures (U.S. DOT \& FAA, 2012). Dynamic pressure $\left(\rho U^{2}\right) / 2$ is the shear stress $\tau_{\mathrm{w}}$, presented in Equation 4, measured in a moving fluid and is dependent on the direction of the motion (Anderson, 2005). The shear stress at the wall boundary is given by:

$$
\begin{array}{ll}
\boldsymbol{\tau}_{\boldsymbol{w}}=\mu(d U / d y)_{y=0} \quad \text { Equation } 4
\end{array}
$$

where $\mu$ is the shear viscosity, (dU/dy) is the velocity gradient at the wall (this will be discussed further in Section 1.5.6), and $y$ is normal to the surface of the fin. This shear stress is due to frictional effects of fluid passing over the surface of the hydrofoil and is measured tangentially on the surface of the hydrofoil (Anderson, 2005). The static pressure $P_{s}$ is a measurement of the fluid pressure at every point on the body's surface, but not associated with the fluid motion (U.S. DOT \& FAA, 2012). Referring to Chapter 3 in the Pilot's Handbook of Aeronautical Knowledge (2008), Figure 3-8 provides images of the static pressure distribution on the surface of a hydrofoil (U.S. DOT \& FAA, 2008). The size of the arrows indicates the magnitude of static pressure at each local point and the direction of the arrows indicates positive or negative pressure values (Anderson, 2005). Arrows pointing outwards correspond to a negative pressure coefficient, while arrows pointing towards the surface of the foil correspond to a positive pressure coefficient. Surface pressure varies with location on the hydrofoil, and these magnitudes change as angle of attack increases (Anderson, 2005). Concurrently, this causes the center of pressure to change. The resultant force is generated from this distribution and unbalance of pressure magnitudes (U.S. DOT \& FAA, 2012). 
Pressure distribution contributes to most of the effect of lift. The differences in pressure across the fin surface cause consequential velocity differences, which contribute to lifting effects (Anderson, 2005). This can be explained by the Bernoulli's principle. The Bernoulli's equation is a statement of the principle of conservation of energy in fluids and Newton's second law (U.S. DOT \& FAA, 2012). This equation describes the total pressure within a moving fluid:

$$
P_{t}=P_{s}+1 / 2 \rho U^{2}
$$

Equation 5

The equation describes the relationship between pressure, density, and velocity for a steady flow of an inviscid, incompressible fluid (NASA, 2014). For a streamline hydrofoil, the density of the fluid remains constant. Therefore, Bernoulli's equation is used to compute the change in pressure as velocity varies (U.S. DOT \& FAA, 2008). For a symmetric hydrofoil at zero degrees angle of attack, the speed and pressure changes are symmetric on both sides of the hydrofoil. As the hydrofoil inclines, the flow lines experience constriction that causes an increase in flow velocity. At a positive angle of attack the hydrofoil produces lower pressure on the topside (suction side) compared to the bottom side (compression side). This is due to a constricted flow path on the suction side, mutually decreasing the static pressure and increasing the dynamic pressure (U.S. DOT \& FAA, 2012). Differences in velocity will occur on each side of an angled fin. For example, at a positive angle of attack an increase in velocity will occur on the topside (suction side) of the fin and a lower velocity will occur on the bottom side (compression side) of the fin. These pressure and velocity differences allow for the fluid to circulate from low-pressure to high-pressure areas at its sharp trailing edges. This area of circulation is known as the stagnation point and is theorized by the Kutta-Zhukhovsky lift theorem. The irrotational flow pattern increases on the hydrofoil are components in the effect of lift.

Amplifying the angle of attack improves the lifting force. For all hydrofoils, there is a critical inclination angle. When exceeded, turbulence will induce drag and the lifting forces will drop. This dramatic reduction in lift causes stall and is discussed further in Section 1.5.4. (Kundu \& Cohen, 2008) 


\subsubsection{WEIGHT}

The weight of a rider and surfboard is the opposing force of lift. It is a force generated by the gravitational attraction of the earth. Weight is a vector quantity that has a magnitude and a direction. The direction of weight is towards the center of the earth. The magnitude is the sum of the weight of the surfer and the surfboard. The stance of the rider on the surfboard will determine the distribution of weight. For the purpose of this thesis, the weight only includes the gravitational force and the weight of the fin.

\subsubsection{THRUST}

Thrust must be greater than drag in order for the surfboard to move forward and increase the surfer's speed (Paine, 1974). In order to maintain a constant speed, thrust and drag must come to equilibrium (U.S. DOT \& FAA, 2012).

\subsubsection{DRAG}

The drag force is the sum of all the forces that resist against the motion of the object (Sadraey, 2009). One of the primary goals in designing transportation devices in aerodynamics and hydrodynamics is to reduce drag (Sadraey, 2009). Drag is considered the opposing force of a rider and surfboard to the direction of fluid motion (Hendricks, 1969). As the fins are the main focus of this thesis repot, drag is the force that opposes the fins movement through water.

In surfing, there are two main components of total drag: friction drag and pressure drag. As discussed in Section 1.5.1, friction drag is due to tangential stresses on the surface of the fin, while pressure drag is due to normal stresses (Kundu \& Cohen, 2008).

There are two types of pressure drags: induced drag and form drag. Form drag is dependent on the shape and orientation of the object in the fluid (Kundu \& Cohen, 2008). It is the result from the pressure differences for producing lift. When considering a hydrofoil with zero angle of attack, there are small changes in pressure across the body. At the leading edge, there is higher pressure due to the blunt impact of the oncoming fluid flow. The leading edge area has low to stagnant velocity, so the pressure is essentially stagnant pressure (Anderson, 2005). As the fluid moves smoothly around the top surface, the 
pressure decreases below the free-stream static pressure, $p_{\infty}$. Near the trailing edge, the pressure increases and this area is called region of adverse pressure gradient. Adverse pressure gradient is the increase in static pressure in the direction of the flow. The velocities within the boundary layers are affected by pressure gradients. As stated earlier, boundary layer velocities are significantly slower compared to the free-stream velocity, due to frictional surface effects. With higher-pressure gradients, the boundary layer velocities will decrease towards zero. Severe enough static pressure gradients can cause the velocities to reverse, in which case flow separation occurs. In adverse pressure gradient area, the pressure distribution over the top surface, $d P / d x$, is positive and its value is small. This means there is no flow serration occurring. The high pressures at the leading and trailing edge are not equivalent. Higher pressure occurs at the leading edge compared to the trailing edge, which can be considered drag due to separation (Anderson, 2005). Pressure distributions are presented in terms of the pressure coefficient $\mathrm{C}_{\mathrm{p}}$ :

$$
C_{p}=\frac{\mathrm{p}-\mathrm{p}_{\infty}}{\frac{1}{2} \rho_{\infty} U_{\infty}^{2}}
$$

Equation 6

At higher angles of attack, the flow separation will increase. There is still a high-pressure stagnant region at the leading edge of the hydrofoil, but the position of this zone has moved. As the fluid moves around the top surface, there is a negative pressure increase compared to the zero degree angle of attack scenarios. The pressure differences between the upper and lower surfaces will decrease, causing lifting values to also decrease. Drag is also increased due to flow separation because the net pressure differences between the leading edge and trailing edge are increased. This is because there is less force acting on the trailing edge, compared to if the flow were attached, opposing the leading edge force (Anderson, 2005). The higher pressure on the bottom surface of the hydrofoiled fin will cause the fluid flow to wrap around the wingtip, towards the surface of lower pressure (U.S. DOT \& FAA, 2008). This causes trailing vortices because of the changing flow speeds and directions (U.S. DOT \& FAA, 2012). The twisting unstable flow patterns are vortices produced along the fin trailing edge (Sadraey, 2009). As the spirals combine, wing tip vortices form. The changing flow speed and direction causes the fluid to deflect downwards behind the trailing edge to induce downwash (U.S. DOT \& FAA, 2008). This downwash combats the upward 
lift force at the trailing edges (Kundu \& Cohen, 2008). These effects intensify as the angle of attack increases. Pressure differences will also increase between each side of the fin. This forms strong vortices and increases induced drag (U.S. DOT \& FAA, 2012). Once the critical angle of attack is reached, there is a major loss in lift, which causes stall.

Induced drag is drag due to tip vortices of a finite wing or fin (Kundu \& Cohen, 2008). The differences in pressure cause lift, but with a finite shape, the fluid tends to leak around the wing tips from high to low-pressure sides (Anderson, 2005). This fluid movement around the tip causes circulatory action that produces vortex motions downstream. The direction of circulation of the trailing vortices opposes the direction of lift, causing a small component of drag to the fin tip. The tip vortices slightly shift the local relative free-stream velocity direction, in the opposite direction of the lifting action, which causes downwash (Anderson, 2005). Due to the alterations in the flow field, pressure distributions change and increase drag. This ultimately affects the angle of attack of the hydrofoil due to the increase in drag, also known as induced drag (Anderson, 2005).

Friction drag is dependent on the velocity and wetted area of the fin (Hendricks, 1969). As fin moves through water, the results are viscous shearing stresses that resist moving water over the surface of the fin (Anderson, 2005). Friction drag is a function of Reynolds number (Sadraey, 2009). As the fluid molecules pass over the surface of the fin, the molecules in direct contact with the surface have a velocity of zero. Moving away from the surface, each layer of molecules moves slightly faster until the free-stream velocity is reached. This laminar area is called the boundary layer. The thickness of this layer is entirely dependent on the viscosity of the fluid and the surface conditions of the fin (U.S. DOT \& FAA, 2008). As the water particles drift closer to free-stream velocity, the flow transitions from laminar to turbulent (Sadraey, 2009).

\subsubsection{LIFT AND DRAG COEFFICIENTS}

Lift and drag coefficients encompass the hydrodynamic components between the water and fin interaction (Sadraey, 2009). This includes water density, object velocity, object reference area $A$, and lift or drag force values. Total drag can be described by calculating the drag coefficient $C_{D}$, while the lift coefficient $C_{L}$ is used to express the total lift of the 
fin (Kundu \& Cohen, 2008). Both the lift and drag coefficients are non-dimensional values, Equation 7 and Equation 8 (Tritton, 1988).

$$
\begin{array}{ll}
C_{L}=F_{L} /(1 / 2) \rho U_{\infty}{ }^{2} A & \text { Equation } 7 \\
C_{D}=F_{D} /(1 / 2) \rho U_{\infty}{ }^{2} A & \text { Equation } 8
\end{array}
$$

The reference area is important when calculating lift and drag coefficients (Sadraey, 2009). The fin area chosen as the reference area for lift and drag coefficients was the profile form view $A=A_{w}$. Figure 1 shows the profile view of the Spotted Dolphin to show the simple fin geometry. The front of the fin shows the leading edge and the back of the fin shows the trailing edge. The chord length is the distance from the leading to trailing edge. This length changes throughout the span of the fin. The span length is the distance from the base to the tip of the fin. An example of a lift coefficient curve and a drag coefficient curve for a NACA0012 airfoil is presented by NASA on the

“Turbulence Modeling Resource” website (Rumsey, 2014). The four curves present experimental comparisons at $\mathrm{Re}=6$ million (Rumsey, 2014).

The lift to drag ratio (L/D) is also another tool used to justify an optimum fin shape or orientation. It is the amount of lift the fin produces compared to the drag value. This provides an assessment of the fins efficiency. A higher L/D ratio indicates a more efficient fin, meaning the fin with the greatest amount of lift and least amount of drag (Gudimetla, Kelson, \& El-Atm, 2009).

\subsubsection{TURBULENCE MODELING AND BOUNDARY EFFECTS}

Turbulence is a 'state of continuous instability' (Tritton, 1988). It is created as the flow of water passes the boundary layer of a hydrofoil. This generally induces the drag by the development of turbulent wake (Lesieur, 2008). In fluid dynamics, turbulence can be characterized by uncertain and chaotic variation of flow motion over time and space (Wahls, 1990). A fluid passing an object initiates turbulence. This causes a disruption in the flow around the object and develops into a turbulent wake (Lesieur, 2008). The fluid motion is a reaction of the fluid and object boundary interaction. The generated turbulence is directly influenced by the movement and characteristics of the boundary walls (Tritton, 
1988). Turbulence is the most complex form of fluid motion because it is difficult to understand, predict, and compute (Wahls, 1990). Inertia of the fluid causes flow instabilities, vortices and chaotic eddies. Inertia effects can be quantified by the Reynolds number $R e$.

Reynolds number is a dimensionless quantity that provides a magnitude value to the flow regime (Zanoun, Durst, \& Nagib, 2003). This value is defined by length and velocity scales, $L_{c}$ and $U_{\infty}$ (Tritton, 1988). For the purpose of this report, $L_{c}$ is represented by each fin's base length and $U_{\infty}$ is the speed at the center of the channel upstream of the fin. The Reynolds number is a ratio of inertial forces to viscous forces; Equation 9.

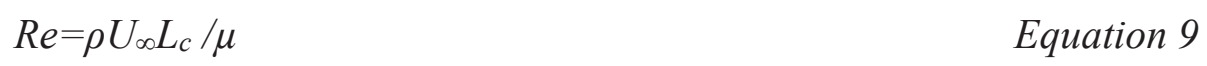

Reynold's number can describe a flow patterns and is an important variable in predicting the lift and drag magnitudes. The flow regimes are characterized as turbulent or laminar. Laminar flow occurs when viscous forces become more dominant than the inertial forces. This provides lower Reynolds number values. As the resistance to the surface of an object amplifies the change of speed and direction for a certain fluid, turbulence can increase. The maximum lift coefficient and the angle at which flow begins to separate are largely dependent on the Reynold's number value. Before transition occurs in low Re value scenarios, the flow separates to produce large wakes behind an angled hydrofoil. High Reynolds numbers provide value to turbulent flow, as inertial forces are dominant. Turbulence in the boundary layer typically occurs at Re $\approx 100000$ (Davidson, 2015). In this case, turbulence will occur before the flow separates and produces smaller wakes. At high $R e$ values, typically $C_{L}$ values will be higher than compared to low $R e$ value flow regimes. (Kundu \& Cohen, 2008) 


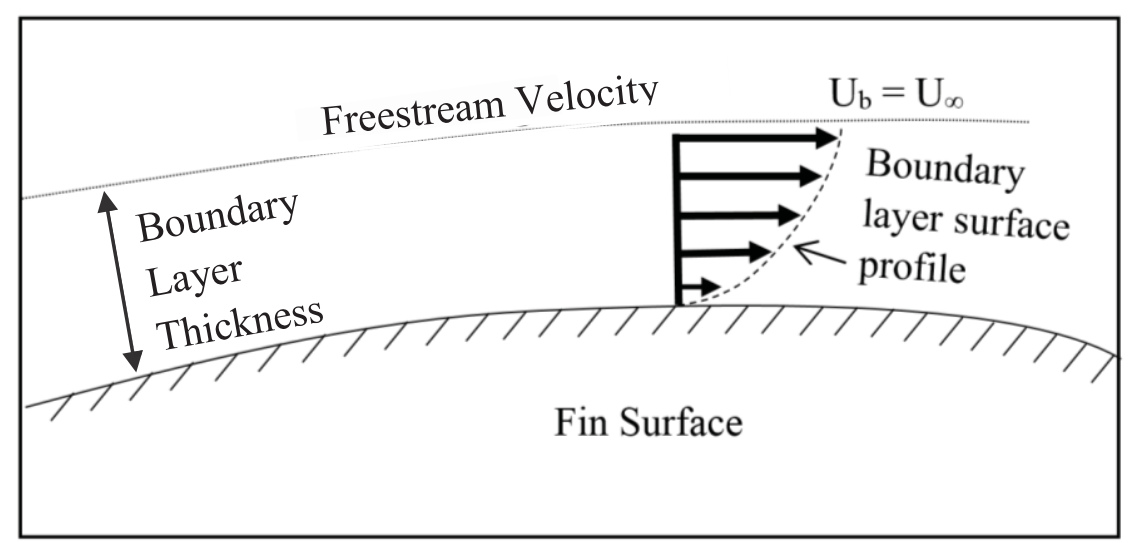

Figure 4: Velocity gradient from boundary layer effects (Image by author, 2015)

By applying NavierStokes based models, two important forces are considered: fluid pressure and shear stress between the object and fluid. Frictional shear force is tangential to the immersed objects in the fluid (Anderson, 2005). Fluid immediately adjacent to the frictional surface is dominated by viscosity and has a no-slip condition (Tritton, 1988). This thin layer near the surface has zero velocity (Denny, 1993). A velocity gradient is shown in Figure 4, depicting the velocity increase in the direction perpendicular to the object's surface (Denny, 1993). Flow velocity changes as a function of distance from zero at the surface (Tritton, 1988). This region is known as an object's boundary layer and can be quantified by the logarithmic law of the wall equation (Zanoun, Durst, \& Nagib, 2003).

Within the boundary layer of a rigid impermeable wall, viscous stresses are exerted directly on the wall of the object. Transitioning away from the wall, Reynolds stresses become more dominant compared to the viscous stresses. Outside of the boundary layer in turbulent water, flow is inviscid and is governed by turbulent pressure forces. Boundary layer thickness is dependent on the fluid properties, velocity, wetted parameter and surface roughness (Denny, 1993). In some cases, a surface is defined with a free slip condition. In this case, the boundary layer gradients are uniform with the mainstream velocity and replicate an open water scenario.

As a hydrofoil increases the angle of attack, the flow boundary can separate from the surface. Flow separation occurs because of adverse pressure gradients and the velocity profile near boundary layers (Anderson, 2005). Near the trailing edge of a hydrofoil, at a high angle of attack, $d P / d \mathrm{x}$ is positive. This means that the fluid particles need to move to 
higher-pressure areas, which exerts more energy. In near boundary layers, the velocity gradients are low due to surface friction. The low energy water particles will slow down to zero velocity when moving closer to the higher-pressure area, and then reverse direction. This change in water particle direction is where the flow boundary separates (Anderson, 2005). The separation of two boundary layers, causes shear layers (Kundu \& Cohen, 2008). Between these shear layers is a dead zone. The separated flow region increases with increasing angles of attack. This ultimately produces more drag and reduces the hydrofoil's lift, causing stall at the critical angle of attack. Flow separation permits a sequence of flow patterns in the trailing wake. The repeating rotational vortices, known as Karman Vortex Street, are due to separated flow instabilities (Kundu \& Cohen, 2008).

Turbulence unpredictability stems from the wide range of mixing lengths (Lesieur, 2008). These flow characteristics must be measured by the fluctuating mixing lengths and velocities. Turbulent flow is governed by applying the Navier-Stokes equations. In CFD simulations, turbulence modeling is important to receive the most accurate results within practical time and computing space limits. There are various classes of turbulence models, some of which include: RANS-based models, large eddy simulations (LES), detached eddy simulations (DES), and direct numerical simulations (DNS). DNS use the Navier-Stokes equations to numerically solve for turbulence without any modeling involved. This computation of turbulence in a flow field is challenging because spatial and temporal scales are extremely small. To calculate and receive accurate results, the grid meshing size must be extremely fine so that computing time is infeasible. To counteract this, Reynoldsaveraged Navier-Stokes equations (RANS) are used in turbulence models. RANS-based models can include additional equations to help solve for turbulence, such as k-epsilon (kع) models; Equation 10 and Equation 11. This two-equation model is used to calculate the energy $k$ and dissipation rate $\varepsilon$ (Warsi, 1993):

$$
\begin{aligned}
& k=\frac{3}{2}(U I)^{2} \\
& \varepsilon=C_{\mu} \frac{\rho k^{2}}{\mu}\left(\frac{\mu_{t}}{\mu}\right)^{-1}
\end{aligned}
$$

Equation 10

Equation 11 
where $I$ is the turbulence intensity (\%), $C_{\mu}$ is $k$ - $\varepsilon$ model coefficient parameter $(0.09)$, and $\mu_{\mathrm{t}}$ is eddy viscosity.

\subsubsection{NEAR WALL TREATMENT}

A challenge in CFD modeling is receiving accurate results in near-wall sub-layers due to viscous effects. Near-wall flow acts differently than flow conditions outside of a surface's fluid boundary. The purpose of a wall function is to calculate these flow conditions without needing extremely fine meshing. Because k- $\varepsilon$ models are not able to predict logarithmic velocity profiles in near wall boundaries, wall functions provide more precise results by using empirical laws. Using $\mathrm{k}-\varepsilon$ models, the wall function approach is considered to avoid inaccurate approximations.

\subsection{LITERATURE REVIEW}

There are only a handful of scientific-based literatures on the mechanisms of surfing. Below, are several articles that have been written about surfing hydrodynamics. Also, in attempt to get a better understanding of the surfboard fin, a few articles on boat keels were reviewed. To help setup the parameters of this project's computational experiments, CFD modeling and turbulence modeling reports are also examined in this section.

\subsubsection{SURFBOARD HYDRODYNAMICS}

Carswell and Lavery (2006) published one of only a few known journal literatures on the topic of surfboard fin design and performance. CAD designed tools and CFD modeling were used in the development and testing of three symmetric single fins. Each fin had the same side and front profiles, but the spanwise direction differed with three base foils investigated: a standard foil, a NACA 4-series and a NACA 6-series. The fins were seperately tested using the FLUENT program by setting the inlet velocity to $0.6-0.7 \mathrm{mps}$ and varying the angles of attack from $0^{\circ}$ to $12^{\circ}$. Turbulence intensity was set to $1 \%$ using the k-epsilon turbulent flow model. Lift, drag, and pressure coefficients were calculated and compared. For this range of incident angles, the lift and drag coefficients were higher than the results found in this thesis. The geometry of the fins and flow parameters also differed. Carswell and Lavery (2006) concluded that the 4-series and 6-series fins produced 
higher lift and lower drag values compared to the standard foil shapes used in todays market. They suggested that profile design might provide a greater impact on the performance of the fin over foil design (Carswell \& Lavery, 2006).

Nicholas Lavery wrote another report in the Reef Journal based on the effects of fillets on fins (Lavery, Foster, Carswell, \& Brown, 2009). The fillets investigated were an un-filleted fin then added fillet radii of $5 \mathrm{~mm}, 10 \mathrm{~mm}, 15 \mathrm{~mm}$, and $20 \mathrm{~mm}$. Three setups were examined at angles of attack ranging from $0^{\circ}$ to $25^{\circ}$ : a single symmetric center fin, a single side fin, and a thruster setup. Inlet velocities ranged from $1 \mathrm{mps}$ to $7 \mathrm{mps}$. A designed CAD program called Dat98 and CFD modeling were used for aquiring the lift, drag, and pressure data. It was found that the addition of fillets provided little improvement to the overall drag of the fins. The study cannot be directly related to the work in this thesis because geometry conditions differed and turbulent flow was not considered.

A CFD study was done through the investigation of three and four fin setup configurations by Gudimetla, et al. (2009). The ANSYS CFX flow solver was used to measure the lift and drag forces, as well as flow visualization around the fins. A turbulence intensity of 5\% was set using the k-epsilon turbulence model. These results were compared to the 7-equation Reynolds stress (RSM) turbulence model. The 3-fin setup produced higher lift and coefficients at lower angles of attack, while the four fin setup had lower lift and drag values at smaller angles of attack. It was concluded that the three fin design was more efficient, higher L/D ratios, which would be most applicable for surfing typical waves. The four fin design was more suitable for big-wave surfing, because the low lift and drag values made the configuration more stable. The visual analysis illustrated the three fin setup inducing tip vortices. Although the geometry and configurations contrasted from fins studied in this thesis, the curves for lift and drag coefficients showed similar trends. The parameters of the study influenced the design of this thesis. (Gudimetla, Kelson, \& El-Atm, 2009).

Sakamoto and Yanamoto (2007) compared a convential fin design to three different modified fins. L/D ratios were used to evaluate the thruster configurations by modeling them in a CFD program called Engineering Fluid Dyanamics ProEngineer. The computational results were compared to visual flow experiments completed in a water tank 
and a quanlitative performance by surfing the fins in the ocean. Dye was used to conduct the visual flow experiments, but no final conclusions were drawn from the laboratory or ocean tests. The quantitative $\mathrm{L} / \mathrm{D}$ ratios did not prove to produce explicit results. (Sakamoto \& Yanamoto, 2007).

\section{BIO-INSPIRED DORSAL FIN PROFILES}

The nine fins chosen for this project were based on species that are considered some of the fastest swimming aquatic mammals and fish in the world (Martin, 2003). A small variety of fish, sharks, dolphins, and whales were used to compare vastly different dorsal fin shapes.

\subsubsection{BLUE SHARK}

The Prionace glauca is known as the blue shark because of its bright blue coloration (Parsons, 2006). The back of this shark is dark blue, the sides are bright blue, and the stomach is a white (Parsons, 2006). Of all shark species, the blue shark is the most widely distributed (Department of Fisheries and Aquaculture, N/A). Their habitat ranges from the topical to temperate oceans (de Azevedo e Silva, 2007). They are typically found near the surface of deeper waters, but rarely swim into shallow inshore waters (Parsons, 2006). This pelagic species is found in the Atlantic, Pacific, and Indian Oceans. Its territory is diverse, reaching from inshore to offshore waters (Department of Fisheries and Aquaculture, N/A).

The blue shark has a slender, bullet-shaped body (Parsons, 2006). The largest blue shark ever recorded was found to have a length of $3.83 \mathrm{~m}$ (Grace, 2001). On average, males grow to lengths of $1.82 \mathrm{~m}$ to $2.81 \mathrm{~m}$ and weights of $29 \mathrm{~kg}$ to $55 \mathrm{~kg}$ (Department of Fisheries and Aquaculture, N/A). Females grow to an average length of $2.21 \mathrm{~m}$ to $3.23 \mathrm{~m}$ (Grace, 2001).

This species of shark has two dorsal fins. The blue shark differs from other members of its family. This is because the dorsal fin is positioned contrary to the common identifying positions of other Carcharhinidae members (Grace, 2001). To distinguish a blue shark, the midpoint of the first dorsal fin is shown to be closer to the pelvic fin than the pectoral fin axil (Cooper, N/A). The first dorsal fin has a very shallow angled leading edge with a rounded apex (Abercrombie, Chapman, Gulak, \& Carlson, 2013).This fin is three times 
shorter than the length of the pectoral fin (Parsons, 2006). The trailing edge has a convex shape with a moderately free rear tip (Abercrombie, Chapman, Gulak, \& Carlson, 2013).

\subsubsection{FIN WHALE}

The Balaenoptera physalus is commonly known as the fin whale and is a member of the Cetacean order (Bannister, Kemper, \& Warneke, 1996). Fin whales are the fastest swimming whale in the Balaenopteridae family (Kinze, 2003). These whale species have documented speeds reaching up to $37 \mathrm{~km} / \mathrm{h}$ (Kinze, 2003). Their habitat range is worldwide, generally in deeper oceanic zones (Bannister, Kemper, \& Warneke, 1996). There are three sub-species of the fin whale: North Atlantic, North Pacific, and Southern Hemisphere (Bannister, Kemper, \& Warneke, 1996).

Based on length, fin whales are the second-largest mammal on earth (NOAA, 2010). Generally, fin whales in the Northern Hemisphere are smaller in length by up to three meters, but are leaner compared to those in the Antarctic (NOAA, 2010). The female can grow $5-10 \%$ longer than the males (NOAA Fisheries, 2013). The Antarctic fin whale length can extend more than 23m (NOAA, 2010). These whale species can live up to 80 to 90 years of age and can weigh more than 70,000kg (NOAA Fisheries, 2013) (NOAA, 2010).

Compared to the blue whale, the dorsal fin is larger (Kinze, 2003). It is located two-thirds of the whale's length from the snout tip (NOAA Fisheries, 2013). Rising in a shallow angle from the body, the dorsal fin has a tall falcate shape (NOAA Fisheries, 2013).

\subsubsection{Short-finned Pilot Whale}

The Short-finned pilot whale, Globicephala macrorhynchus, is a member of the Cetacean family Delphinidae (Bannister, Kemper, \& Warneke, 1996). They can be found in tropical and warm-temperate waters, as well as extending into cold-temperate waters in the North Pacific (IUCN/SSC Cetacean Specialist Group, 2003).

Short-finned pilot whales are among the larger species within the dolphin family. Their size varies within the species and there is a size difference between the genders. The males are longer in length, with an average length of $5.5 \mathrm{~m}$. They can grow up to $7.3 \mathrm{~m}$ in 
maximum length. The females have an average length of $3.7 \mathrm{~m}$. The weight ranges from 1000 to $3000 \mathrm{~kg}$. (NOAA Fisheries, 2012)

The dorsal fin is set forward on the back of the short-finned pilot whale (NOAA Fisheries, 2012). It is located about one-third of the way back from the tip of the snout. The base of the dorsal fin is relatively broad. (Department of the Environment, 2015)

\subsubsection{DALL'S PORPOISE}

The dall's porpoise, Phocoenoides dalli, is a fast swimming member of the Phocoenidae family (IUCN/SSC Cetacean Specialist Group, 2003). They are the fastest swimming small cetaceans, with speeds up to $55 \mathrm{~km} / \mathrm{h}$ over short distances (NOAA Fisheries, 2012). They are commonly found in temperate to boreal waters. They're distributed across the North Pacific Ocean and adjacent seas (IUCN/SSC Cetacean Specialist Group, 2003). Within this range, the dall's porpoise habitat includes oceanic waters offshore, inshore, and near-shore (NOAA Fisheries, 2012).

Sizes vary depending on individual, population, and gender. Populations in the eastern pacific tend to be smaller than western pacific. The male dall's porpoise are typically larger than the female. The males can grow up to lengths of $2.4 \mathrm{~m}$, while the females can grow up to $2.2 \mathrm{~m}$ (Jefferson, Leatherwood, \& Webber, 1993). A dall's porpoise can weigh up to $220 \mathrm{~kg}$ (NOAA Fisheries, 2012).

The dorsal fins are located in the middle of their back. Shapes differ by individual and gender, but are predominantly triangular shaped with a wide base (Jefferson, Leatherwood, \& Webber, 1993). The fins often cant in a more forward projecting position in the males compared to the females. (NOAA Fisheries, 2012)

\subsubsection{PANTROPICAL SPOTTED DOLPHIN}

The Stenella attenuata is commonly known as the pantropical spotted dolphin. It is a member of the Cetacean family Delphinidae. The English name derives from the spotted patterns on adults. Coloration and skeletal characteristics does vary significantly over their habitat distribution. As the name pantropic implies, these species are very widely distributed. They are found in oceanic pelagic zones, as well as within near-shore habitats 
(IUCN/SSC Cetacean Specialist Group, 2003). Pantropical spotted dolphins can be found in a range from tropical to subtropical waters, and occasionally temperate waters (Bannister, Kemper, \& Warneke, 1996). These ranges include the Atlantic, Pacific, and Indian Ocean (Mottet, N/A).

The biological characteristics differ between the female and males. Females can grow to a length of $2.4 \mathrm{~m}$ while the males can only grow to about $2.57 \mathrm{~m}$ in length. These dolphin species generally sustain a maximum weight of $119 \mathrm{~kg}$ and can live up to 50 years in age. (Bannister, Kemper, \& Warneke, 1996)

The dorsal fin has a tall falcate shape, which is very narrow and pointed (WDC, N/A). These fins will sometimes have a distinct backwards-curving arch. This creates a convex leading edge and a deep concave trailing edge. In some pantropical spotted dolphins, the leading edge of the dorsal fin can be nearly straight. Tips can vary from slightly pointed to slightly round. These characteristics vary between genders and habitat range. (Mottet, N/A)

\subsubsection{Killer Whale}

Killer whales, Orcinus Orca, are Cetacean's belonging to the Delphinidae family (Jones, 2006). They have the widest population habitat range, but are most abundant in colder waters (Pitman, Perryman, LeRoi, \& Eilers, 2007). They are common in the waters of Antarctica, North Atlantic and Pacific Oceans. Although they are scarcer, killer whales can also be found in tropical and subtropical regions (NOAA Fisheries, 2014). The most studied populations include the northeastern Pacific Ocean, which consist of three ecotypes: transient, residential, and offshore (Jones, 2006). Each population differs in diet, genetically, morphologically, behaviorally, socially, and ecologically (NOAA Fisheries, 2014) (Jones, 2006).

The killer whale is the largest member of the Delphinidae family (Department of the Environment, 2015). Size dimorphism is a trait shown in this species, meaning the males grow considerably larger than the females (NOAA Fisheries, 2014). The male Orca can grow up to $10 \mathrm{~m}$ in length and can reach weights of $10000 \mathrm{~kg}$. The female can grow up to about $8.5 \mathrm{~m}$ in length, while weighing $7500 \mathrm{~kg}$ (NOAA Fisheries, 2014). 
One of this species' most distinguishing features is their unique dorsal fin. This fin is tall and wide in both genders of the killer whale species. Males have an upright, more pointed and triangular shaped dorsal fin compared to the females more curved fin (Department of the Environment, 2015). These fins also vary between the different populations. The residential killer whales have a falcate shape with a more rounded tip. Comparatively, the dorsal fin of the transient killer whale is straighter at the tip. The offshore population tends to be smaller in overall size. (NOAA Fisheries, 2014)

\subsubsection{SHORTFIN MAKO SHARK}

The shortfin mako, Isurus oxyrinchus, is a member of the Lamnidae family. They are widely distributed and common in the Pacific, Atlantic, and Indian Ocean. This pelagic, coastal and oceanic shark typically occurs in temperate and tropical waters. They can be found near the surface or in deep water, up to $400 \mathrm{~m}$ in depth. (Kabasakal \& Kabasakal, 2013)

The shortfin mako shark can grow up to a maximum length of $3.7 \mathrm{~m}$, but typically range between $1.5 \mathrm{~m}$ and $2.5 \mathrm{~m}$ (Department of Fisheries and Aquaculture, N/A). Most individuals weigh about $60 \mathrm{~kg}$ to $135 \mathrm{~kg}$, but females tend to be larger than males. The female can grow to a maximum size of $3.8 \mathrm{~m}$ in length and about 570kg (Passarelli, Knickle, \& DiVittorio, N/A).

The dorsal fin is very erect from the body of the mako shark. The leading edge has a steep angle with a rounded apex (Abercrombie, Chapman, Gulak, \& Carlson, 2013). The trailing edge is moderately straight with a short free rear tip (Abercrombie, Chapman, Gulak, \& Carlson, 2013).

\subsubsection{SWORDFISH}

Xiphias gladius, part of the Istiophoridae family, are known as the swordfish or broadbills (FAO Fisheries, 1985). They are one of the fastest swimming predators in the world (NOAA Fish Watch, 2015). These oceanodromous species have worldwide distribution (de Azevedo e Silva, Azeredo, Lailson-Brito, Machado Torres, \& Malm, 2007). Their habitat range includes the Pacific, Atlantic, and Indian Oceans (FAO Fisheries, 1985). 
They can be found in both temperate and tropical areas, but sometimes reaching colder waters (NOAA Fish Watch, 2015). They are epi- and mesopelagic fish, which can be found in the deep ocean, and occasionally near shore. There are two categories of swordfish, the North-Atlantic and Pacific swordfish (FAO Fisheries, 1985).

Their streamlined body shape allows them to swim up to 50mph (NOAA Fish Watch, 2015). These fish can reach a maximum total length of $445 \mathrm{~cm}$ at maturity (FAO Fisheries, 1985). They can weigh up to 540kg (FAO Fisheries, 1985). The size of the swordfish depends on their habitat. Swordfish in the northwestern Pacific tend to be larger than their counterparts. Females are usually larger in body size compared to the males (FAO Fisheries, 1985).

The swordfish has two dorsal fins; the first is well separated from the second dorsal fin. Both dorsal fins are supported by a series of spines called rays. The average mature swordfish has 34 to 49 soft rays in the first dorsal fin and four to six soft rays in the second (FAO Fisheries, 1985). The first dorsal fin has a crescent-like shape. It is tall in height, but has a short base length (NOAA Fish Watch, 2015).

\subsubsection{BLUE MARLIN}

The Makaira nigricans are members of the Istiophoridae family. These fish are commonly known as blue marlins (FAO Fisheries, 1985). Their territory range is mainly in the tropical and temperate waters of the Atlantic Ocean (NOAA Fish Watch, 2015). They mainly occur between $30^{\circ} \mathrm{S}$ and $45^{\circ} \mathrm{N}$ (Gardieff, N/A). Blue marlins are pelagic-oceanic species (NOAA Fish Watch, 2015).

Blue marlins can grow to lengths of $375 \mathrm{~cm}$. They are the largest of the Makaira. The maximum weight is $580 \mathrm{~kg}$, but they have an average weight between $136 \mathrm{~kg}$ and $181 \mathrm{~kg}$. (FAO Fisheries, 1985) Females are generally larger than males (Gardieff, N/A).

There are two dorsal fins; the first is larger than the second dorsal fin. The base of the first fin is long and it extends close to the second dorsal fin. The leading edge is set forward and has a high pointed tip (Gardieff, N/A). The trailing edge slopes steeply and then throughout the length of the base, its height is lower than the maximum body depth. 


\section{Chapter 2: CFD Experimental Setup}

\subsection{OPERATIONAL SYSTEM}

All fin design modeling and simulations were performed on a Dell Precision T1700 computer with a Windows 7 Enterprise 64-bit operating system. The system operated on an Intel ${ }^{\circledR}$ Core ${ }^{\mathrm{TM}}$ i7-4770 $\mathrm{CPU}$ processor at $3.40 \mathrm{GHz}$ and $8.00 \mathrm{~GB}$ of RAM. This computer was provided by Michigan Technological University.

\subsection{Modeling Programs}

\subsubsection{MICROSOFT EXCEL}

Microsoft Excel was used to calculate, organize, and scale each of the fins' attributes. The measured data points for the outline of each fin profile were input into Excel. These points gave distance values to project the hydrofoil size and positioning. Excel was also used to scale all profile data to the appropriate size defined by the base length of the fins. Hydrofoil data was downloaded and stored in Excel for scaling purposes.

All numerical data pertaining to the result outputs were stored in Excel. This information was organized to create tables and figures for the purpose of this thesis analysis.

\subsubsection{SOLIDWORKS}

The SOLIDWORKS program is a product of the Dassault Systemes. It is a threedimensional solid modeling computer-aided design (CAD) software (Dassault Systemes, 2015). The 2013 Education Edition, for 64-bit Windows, was used in the design of the 3D fins. Each fin was equipped with a series of two-dimensional hydrofoils attached to a spline. The "Loft" tool was used in order to project these 2D drawings into a 3D model. All models were saved as Parasolid files in order to import into the NX modeling program.

\subsubsection{SIEMEN'S NX 9.0}

The program Siemen's NX 9.0 is a computer-aided manufacturing (CAM), design (CAD), and engineering (CAE) software package (Siemens PLM Software Inc., 2015). The NX CAE package was used in the NX solutions for simulation district of the program. This 
was used in order to process and analyze computational fluid dynamic (CFD) models. Specifically, the CFD models administered fluid-flow simulations to measure the flow field, lift and drag forces. Preprocessing was completed within this program to provide boundary and flow values in order to complete the simulation solver. The simulation solver applied in NX was the FLOW solver. The flow field was analyzed for each of the ten fins for each angle of attack, $0^{\circ}$ to $45^{\circ}$. The lift and drag forces for each simulation were also obtained using this program's outputs. Turbulence values and locations were evaluated in the post-processing reports.

\subsubsection{TURBULENCE MODELING}

The time-averaged RANS-based model was applied to compute the Reynolds stresses. Accompanying this modeling method was the k-epsilon model. This two-equation model is a type of linear eddy viscosity (LEV) model that solves for turbulence. The turbulent kinetic energy $(\mathrm{k})$ equation calculates the energy in the turbulence $\left(\mathrm{m}^{2} / \mathrm{s}^{2}\right)$. The turbulent dissipation $(\varepsilon)$ equation calculates the scale of the turbulence $\left(\mathrm{m}^{2} / \mathrm{s}^{3}\right)$. 


\section{Chapter 3: Laboratory Experimental Setup}

\subsection{HYDRODYNAMICS LABORATORY}

The laboratory used for the experimental portion of this thesis was located in room 110 in Dillman Hall at Michigan Technological University. A closed loop water channel was built in this hydrodynamics lab. All lab experimental testing and data were gathered in this flume.

\subsection{Water Channel Setup}

\subsubsection{WATER CHANNEL FEATURES}

The water channel (Figure 5 through Figure 8) was built with cement blocks in a rectangular shape, which was attached to the concrete floor (Champagne, 2011). This flume consisted of sections including the water inlet, flow straighteners, a flow-development section, the testing zone, a float valve depth controller, and an outlet zone. Metal rails were built to the length of the water channel for the use of mounting and moving instruments up and downstream of the testing section.

The inner-wall dimensions had a width of $0.92 \mathrm{~m}$, a total height of $1.11 \mathrm{~m}$, and a total inside length of $10.18 \mathrm{~m}$. To prevent leakage, the walls and floor were coated with a 1/16-inch thick rubber membrane. The experimental tank was comprised of a closed loop flow system with an inlet and outlet section in order to re-circulate the water.

The inlet section had dimensions of $0.93 \mathrm{~m} \times 0.92 \mathrm{~m} \times 1.11 \mathrm{~m}$. The $0.25 \mathrm{~m}$ diameter inlet pipe was located at the head of the tank. This PVC pipe connected the pump in the outlet section to the inlet section in order to make the water channel a closed loop system. The pipe was equipped with a manometer to measure the difference in head heights. The inlet section of the pipe had 16 holes to allow for even water discharge. An internal wall was constructed between the inlet and main experimental tank sections, which was $0.70 \mathrm{~m}$ in height from the ground. A series of small diameter PVC flow-straightening pipes were installed on top of this dividing section; the stacked pipes were $20 \mathrm{~mm}$ in diameter and $0.30 \mathrm{~m}$ in length. These pipes were assembled parallel to flow to allow for flow straightening into the flow 
development section. Due to the high velocities during the experiments, and because the diameter of the flow straighteners were extremely small, water backup in the inlet section became an issue. This caused an increase in water height in the inlet section, above the experimental section water height. A consequential waterfall effect caused high turbulence in the flume. To fix this issue, a rubber mat was installed $0.43 \mathrm{~m}$ from the flow straighteners to calm the water surface fluctuations.

A flat bed was constructed following the flow straightener section. This bed extended the full length of the flume, until the outlet section. The experimental section had a length of $8.04 \mathrm{~m}$ and stood $0.70 \mathrm{~m}$ in height at zero degrees slope. The bed and supports were made of plywood and 2x4" wood blocks. A layer of primer and sealant was applied to the wood to protect against rotting. An epoxy varnish overlay was applied to the top surface of the bed and cracks were sealed with a sealant. At zero velocity, the water was filled to a height of $205 \mathrm{~mm}$ from the flat bed.

The experimental section spilled into the outlet section, where the outlet pipe connected to the pump system. Like the inlet section, the outlet area had dimensions of $0.93 \mathrm{~m} \times 0.92 \mathrm{~m}$ $\mathrm{x} 1.11 \mathrm{~m}$. The $0.25 \mathrm{~m}$ diameter outlet pipe fed water into a 20 -HP centrifugal pump, which was controlled by a variable-speed electric controller. The velocity of water was measured using two systems: a two-tube manometer and an Acoustic Doppler Velocimeter (ADV). The ADV was positioned $2.00 \mathrm{~m}$ from the rubber flow straightener, near the inlet section, in the middle of the water channel; it was $0.46 \mathrm{~m}$ from the sidewall. The experimental area was $2.54 \mathrm{~m}$ downstream of the ADV, so the surfboard and fin system were positioned in front of the window.

The Cartesian coordinate system was used to assign the distances, directions, and positions of each aspect of the water channel setup. The water moved from the inlet to the outlet section in the positive X-direction. The horizontal width of the water channel, from the window to the surfboard was in the positive Y-direction. Vertically, from the top of the water channel to the floor was in the positive Z-direction. 


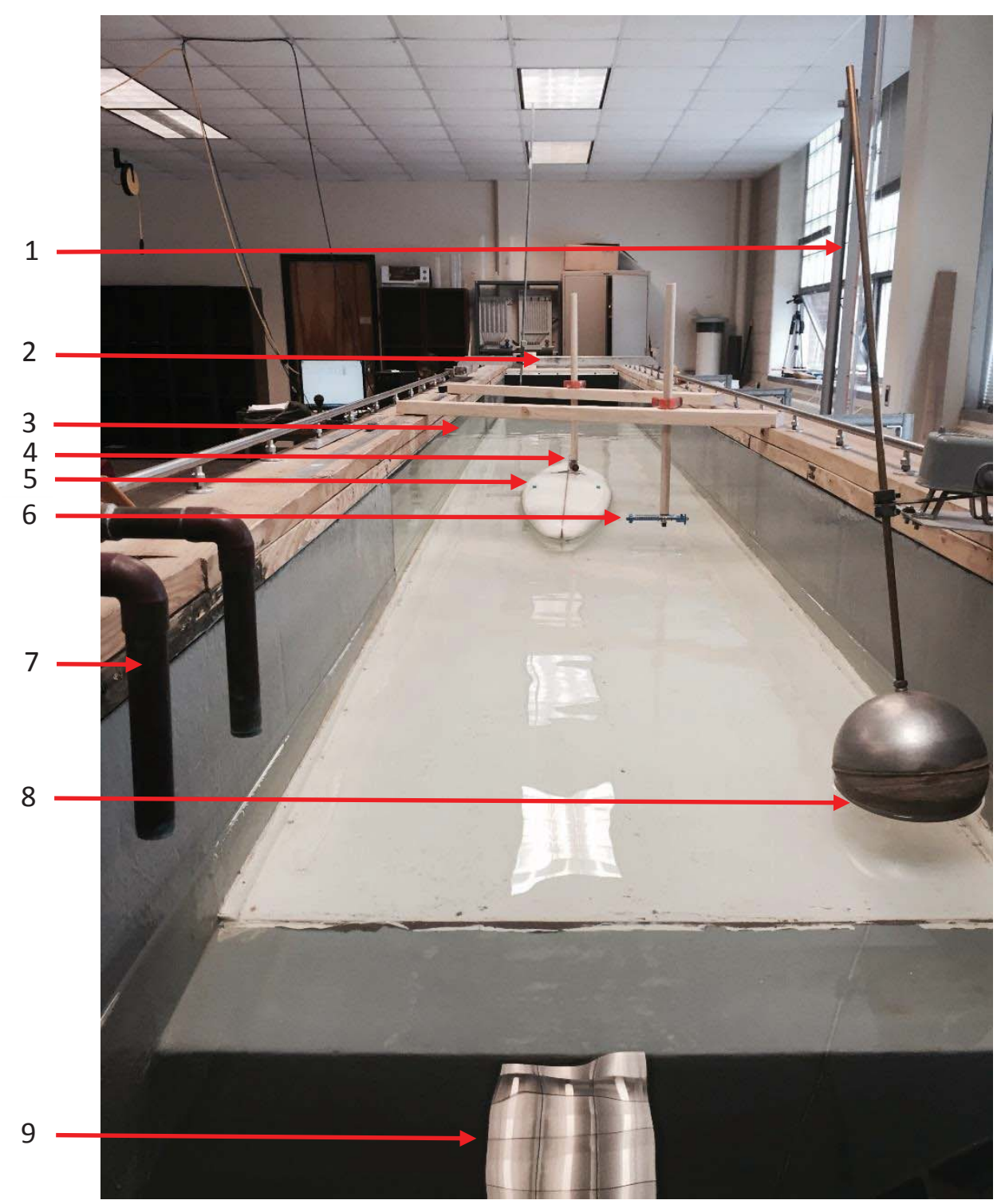

Figure 5: View of the water channel and experimental setup from the downstream (outlet) section. List below indicates feature details. (Image by author, 2015)
1 Manometer
2 Inlet section (see Figure 6)
3 Window
4 Spring gauge and holding system (Drag force)
5 Surfboard
6 Spring gauge and holding system (Lift force)
7 Water supply
8 Float valve
9 Outlet section (see Figure 7) 


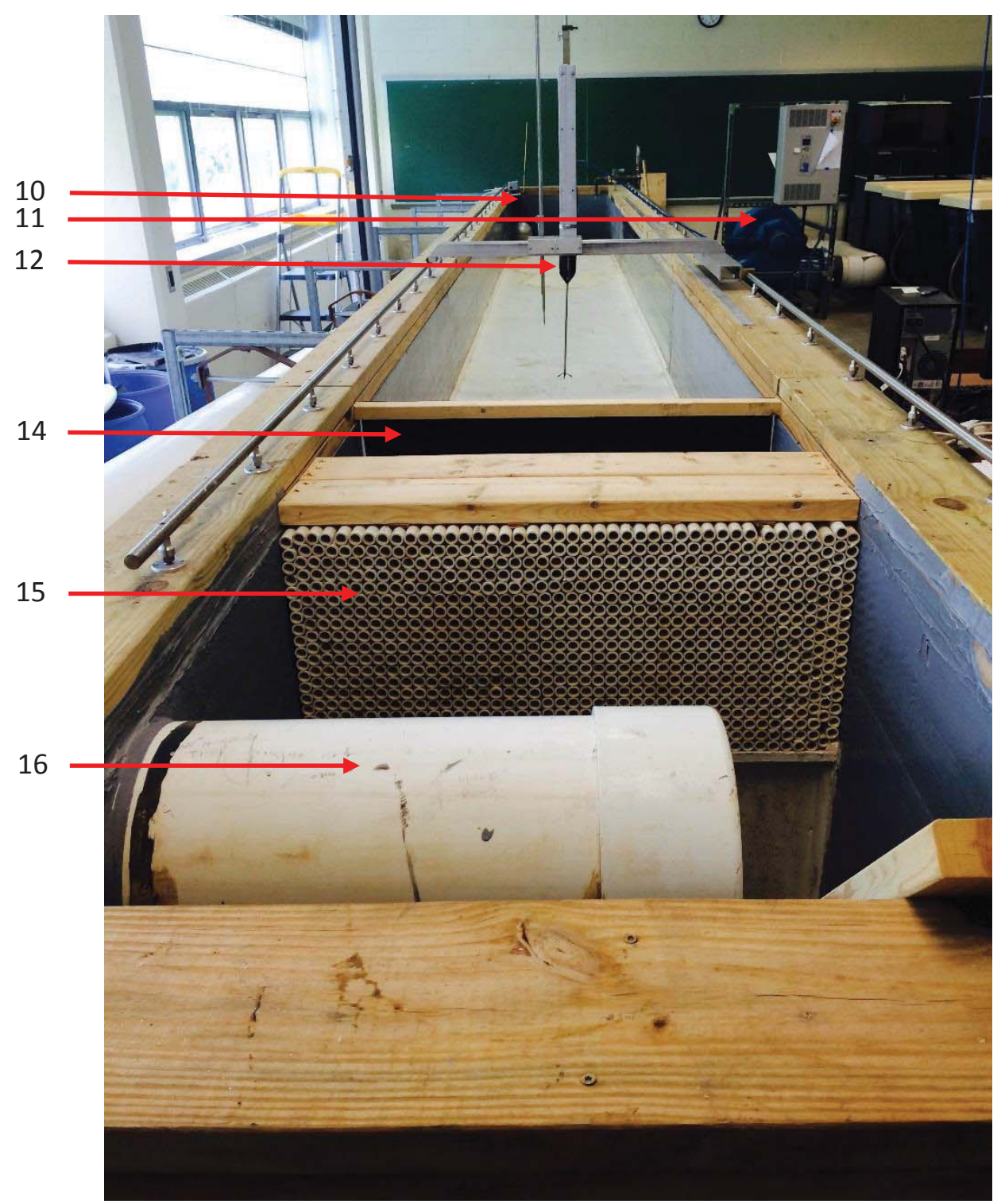

Figure 6: View of the water channel and experimental setup from the upstream (inlet) section. List below indicates feature details. (Image by author, 2015)

10 Outlet section (see Figure 7)

11 Pump

12 ADV and instrument carriage

13 Instrument carriage track

14 Rubber piece- Flow straightener

15 PVC pipes- Flow straightener

16 Pipe inlet from pump and Inlet section 


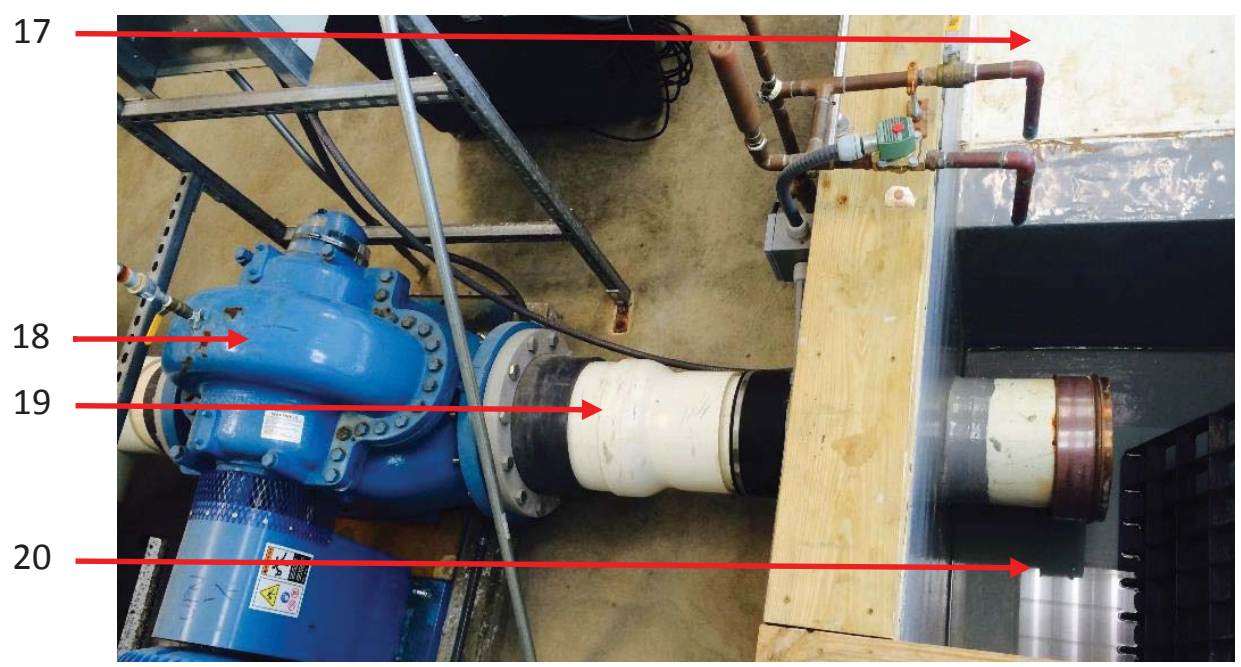

Figure 7: Plan view of the pump and water channel (outlet section) setup. List below indicates feature details. (Image by author, 2015)

17 Experimental section (see Figure 8)

18 20-HP Pump

19 Pipe outlet to pump

20 Outlet section

\subsection{SURFBOARD AND FINS}

\subsubsection{SURFBOARD}

The replicated surfboard, to be used in these experiments, was based off of the Gerry Lopez 1975 Lightning Bolt. Gerry Lopez shaped and designed the board as a flyer pintail. This sleek longboard was intended for a single fin setup (SURFER Magazine, N/A). It measured a length of $2.44 \mathrm{~m}$ and a width of $47.0 \mathrm{~cm}$ at the broadest point. The silhouette formed a medium rocker and a flat deck. The nose was designed as a pin shape with corresponding width dimension of $30.5 \mathrm{~cm}$. The edges formed soft rails across most of the surfboard's length, but developed into hard rails near the tail. The surfboard had a maximum thickness of $7.3 \mathrm{~cm}$, tapering near the edges. The tail was molded into a flyer pintail shape at $22.9 \mathrm{~cm}$ wide. The initial single fin position was placed $14.0 \mathrm{~cm}$ from the tail. (Cater, 2013)

The surfboard was scaled down to fit the water channel. A 1:3 ratio of the water channel width was used to define the scaled width dimension of the surfboard. Using this ratio 
based on the water channel width $(0.92 \mathrm{~m})$, the surfboard width was set to $30.7 \mathrm{~cm}$. All other surfboard dimensions were based off of the original surfboard width to scaled surfboard width ratio. The reproduced Lightning Bolt surfboard was built by the author of this paper and the shapers at Chopstix in Chassell, MI. The dimensions were scaled and calculated to determine a length of $1.59 \mathrm{~m}$ and a width of $30.7 \mathrm{~cm}$; approximately 1.5 times smaller than the original Lightning Bolt surfboard dimensions.

\subsubsection{FINS}

The fin designs used in these experiments were based off of nine bio-inspired dorsal fin shapes. The fin parameters consisted of an initial base length of $112 \mathrm{~mm}$ and $177.8 \mathrm{~mm}$ height. Appendix B provides images of each original designed fin and full-scaled images; Figure 91 through Figure 99. The hydrofoil used was the four-digit series NACA 0012. The " 00 " represents a foil with a symmetric shape and no camber. The " 12 " gives value to a $12 \%$ thickness of the chord length. Each fin had a different profile shape, deriving from the dorsal fin profile shapes of nine different aquatic species. Due to wall boundary effects from the water channel, the full-scaled fins were scaled down and printed to fit the water channel. The dimensions of these flume-scaled fins were $73.09 \mathrm{~mm}$ base length and $166.04 \mathrm{~mm}$ height.

\subsubsection{D PRINTER}

The 3D printer used to print each fin and the fin insert system was the Ultimaker 2. The associated software program, Cura, was used to import the STL files for formatting purposes. The filament used for each print was a $2.85 \mathrm{~mm}$ diameter PLA (Polylactic acid) polymer. The blue PLA plastic came on a reel with approximately $90 \mathrm{~m}$ of material. The print temperature was set to $210^{\circ} \mathrm{C}$ with all fans turned on. The plate temperature was set at $70^{\circ} \mathrm{C}$. For higher quality results, the standard print speed was reduced to $50 \mathrm{~mm} / \mathrm{s}$. To save on time and material, the interior of the fins were set for a $20 \%$ infill; this value gave enough stability for the purpose of these experiments. The full-scaled fin print took approximately seven to eight hours per fin. Each flume-scaled fin print took approximately four to five hours per fin. 


\subsubsection{FIN MATERIAL}

Polylactic acid (PLA) is a thermoplastic material that is biodegradable (Tokiwa, Calabia, Ugwu, \& Aiba, 2009). PLA has a melting point at $200^{\circ} \mathrm{C}$, but it does harden once cooled (Garlotta, 2001). This material was chosen based on its high strength and ease of processing to construct the fins. The PLA was purchased from the Ultimaker 2 distributor. It was 90m long with a $2.85 \mathrm{~mm}$ diameter and was packaged on a reel for printing convenience (Ultimaker, 2015). A light blue color was chosen for contrast against floor of the water channel.

Once the fins were printed, two coats of Sikkens Colorbuild Plus were applied (Akzo Nobel Coatings Inc., 2008). This was done to provide a smooth surface to the fin and to seal the fin to make it water resistant.

\subsection{DAta Collection}

\subsubsection{Positioning SySteM}

The support structure that held the surfboard and force gauges in position was fastened to the top of the water channel; this system is shown in Figure 8. Two wooden boards were placed on top of the channel. The leading wooden board was placed across the water channel, upstream of the measurement area. The second wooden board was secured downstream, above the tail of the surfboard. A wooden dowel accompanied each wooden board, as shown in Figure 8(C). These dowels were drilled into the center of the wooden board and braced the force gauges at the lower tip. The trailing wooden board and dowel held the spring gauge that measured lift force, as demonstrated in Figure 8(A). Figure 8(B) shows the leading board and dowel, which held the spring gauge that measured drag force. 

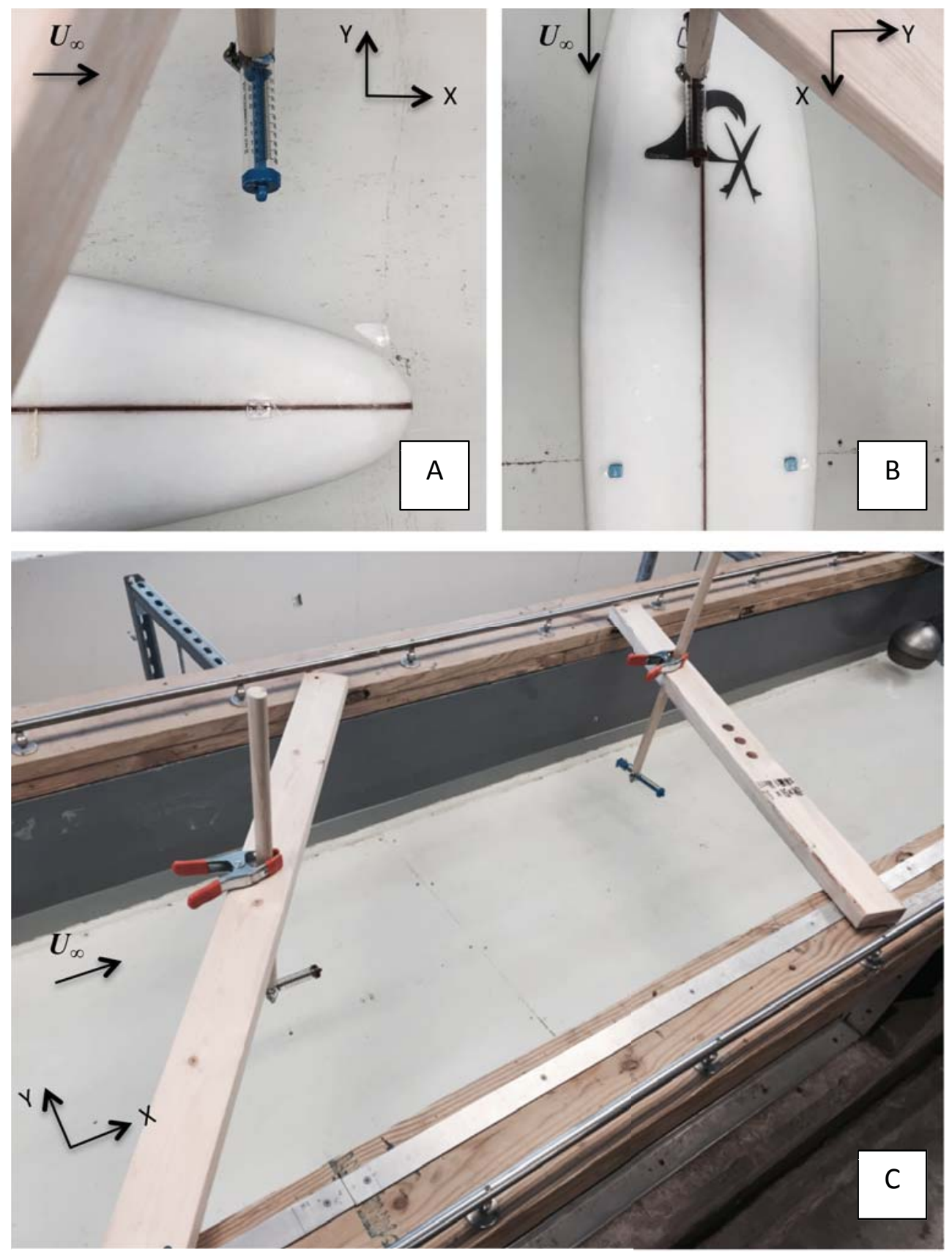

Figure 19: Experimental section (A) Plan view of surfboard and spring gauge- measuring lift force (B) Plan view of surfboard and spring gaugemeasuring drag force $(C)$ isometric view of the positioning system (Image by author, 2015) 


\subsubsection{FORCE GAUGES}

The instruments used were non-electric spring loaded meters that measured the surfboard and fin's magnitude of force. This mechanical force gauge was calibrated in order to receive accurate lift and drag measurements. Two different spring balances were used to measure in units of Newton. These force gauges, shown in Figure 9, ranged from 0-1N (red on the left) and 0-2N (blue on the right).

\subsubsection{DYE INJECTIONS}

A syringe with an attached copper tube was used to shoot the dye in front of the fin. The purpose of the thin copper tube was to create the least amount of disturbance in front of the fin in order to receive accurate flow conditions. The dye used to analyze the flow behavior was yellow food color from Gordon Food Service.

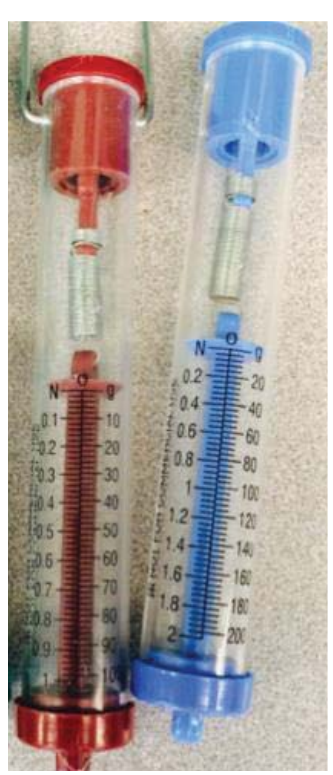

Figure 9: Spring gauges used for the lab lift and drag force (Image by author, 2015)

\subsubsection{PHOTOS AND VIDEOS}

The iPhone 6 was used to capture pictures of all setups and laboratory work. It was also used to take slow motion video of the visual flow analysis portion of the laboratory work. The camera on the iPhone 6 captured 1.5-micron pixel pictures and 240 fps slow motion video. (Apple, 2015)

\subsubsection{ADV}

The Acoustic Doppler Velocimeter (ADV) flow measurement tool was used to take samples of the flow velocity and turbulence magnitude during the experimental work. It was used for both the visual analysis portion as well as the lift and drag measurements. The ADV collects instantaneous 3D velocity data. It is a Nortek Vectrino Velocimeter as it is manufactured by Nortek AS (Nortek, 2015). The ADV collects data by sending out an acoustic signal through a probe in a cylindrical volume. The echo of the signal is collected and the Doppler shift is measured. As the flow carries small dust particles through this cylindrical volume, sound is reflected and the velocity of these particles are measured. The 
assumption is, the particles in the fluid are flowing at the same rate as the water (Barlock, 2013).

The ADV is positioned on an instrument carriage, upstream of the surfboard and fin setup. The carriage runs the width of the flume, but stays in the upstream position for the purpose of these experiments. Figure 6 shows the ADV in the water channel. 


\section{Chapter 4: CFD Work for Single Fin}

Computational modeling is a tool that can be used to investigate the surfboard system and designs through the isolation of individual variables. In this thesis report, the single variable analyzed was a profile shape of a single fin at changing angles of attack. The study focuses on the complex variables of lift and drag forces, as well as fluid flow around a fin. The lift and drag of the hydrofoil depends on the fins angle of attack, wetted area of the fin, and fluid density and velocity. Using Siemen's NX flow simulator, fluid dynamic forces were investigated around nine different single fin setups. These results and comparisons are presented within this section of the thesis report; Section 4.6 and Section 4.7.

\subsection{FIN DESIGN}

The selected marine mammals and fish, for this project, are among the world's fastest swimming species. Photographs of the profile for each dorsal fin were gridded in one-centimeter squares. Within each square, $\mathrm{X}$ - and $\mathrm{Y}$ coordinate points were measured from an origin and were input into an Excel spreadsheet. This gridding system was used to delineate the anatomical characteristics of the profile shape. This information allowed for the creation of a two-dimensional (2D) spline in the program Solidworks for each fin profile. The points also provided base length dimensions for each hydrofoil, and associated Z-direction positioning

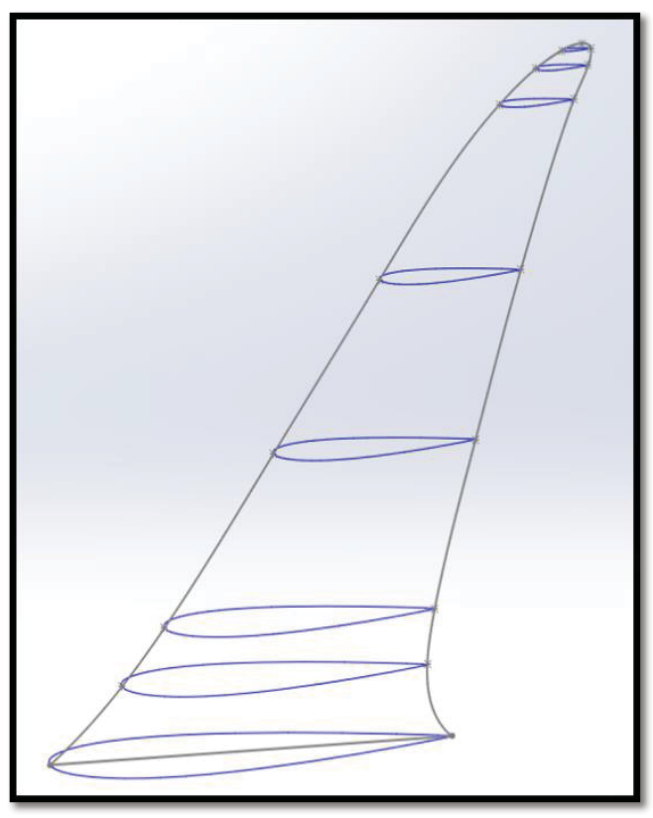

Figure 10: Spotted Dolphin spline and foil shapes (Image by author, 2015)

on the spline.

The hydrofoil data was downloaded from UIUC Airfoil Coordinates Database website into an Excel spreadsheet (UIUC Applied Aerodynamics Group, 2015). Several hydrofoils were associated to each fin's spline shape to project a three dimensional object. An example 
of a spline and attached hydrofoils is illustrated in Figure 10. Each hydrofoil was scaled to the length of the spline, depending on the position in the Z-direction. Microsoft Excel was used to organize the shape values from the gridding system and to compute the ratios to a fin base length of $112 \mathrm{~mm}$. Because of this, fin heights ranged from 48 to $166 \mathrm{~mm}$. This provided each fin with a different profile shape, height, and sweep angle.

To build a three-dimensional (3D) solid model, a 2D foil shape was imported and scaled to the spline outline; Figure 10. The $\mathrm{X}, \mathrm{Y}$, and $\mathrm{Z}$ data points were scaled to the spline base length and position. The foil chosen was the four-digit series NACA 0012, originating from (Lavery, Foster, Carswell, \& Brown, 2009). Its shape is symmetrical, based on the " 00 " digit values, with a $12 \%$ thickness of the chord. This foil was used for all ten of the single fins. The base foil chord length was $L_{c}=112 \mathrm{~mm}$ to fit the base length of the proposed fin (Gudimetla, Kelson, \& El-Atm, 2009) (Lavery, Foster, Carswell, \& Brown, 2009). The spline contained multiple foils distributed throughout the height of the profile in order to loft an accurate 3D model of the aquatic dorsal fin. Due to the range of fin heights produced from this method, the solid fins were all scaled to a height of $\mathrm{L}_{\mathrm{w}}=177.8 \mathrm{~mm}$. This was based on an average single fin height found from several large fin companies (Futures Fins, FCS, etc.). By fixing the height and base dimensions, the unique shape of each fin profile was distorted. Although the shapes were inspired by biological influences, this manipulation could have changed the natural advantages of each fin shape.

The proportions of the full-scaled fins were sized down to fit the water channel dimensions and labeled flume-scaled. Table 1 provides original fin heights $\left(\mathrm{L}_{\mathrm{w}}\right)$ before scaling as well as surface area $\left(A_{s}\right)$, aspect-ratio $(A R)$, volume $(V)$, and wing area $\left(A_{w}\right)$ values for each fin at original, flume-scaled, and full-scaled dimensions. 
Table 1: Dimensions of original, flume-scaled, and full-scaled fins for nine fin types (Table by author, 2015)

\begin{tabular}{|c|c|c|c|c|c|c|c|c|c|c|}
\hline & & BS & SD & FW & SF & SFPW & $\mathbf{M}$ & $\mathbf{O}$ & DP & SFM \\
\hline \multirow{5}{*}{ 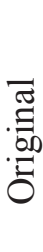 } & $\mathrm{L}_{\mathrm{W}}(\mathrm{mm})$ & 74.9 & 77.5 & 75.9 & 116.9 & 48.4 & 83.8 & 166.3 & 67.3 & 108.5 \\
\hline & $\mathrm{A}_{\mathrm{W}}\left(\mathrm{cm}^{2}\right)$ & 42.2 & 39.6 & 38.8 & 55.9 & 34.6 & 38.1 & 88.5 & 39.3 & 66.7 \\
\hline & $\mathrm{A}_{\mathrm{s}}\left(\mathrm{cm}^{2}\right)$ & 98.0 & 93.3 & 91.6 & 126.4 & 83.7 & 89.2 & 191.9 & 91.8 & 147.7 \\
\hline & $\mathrm{V}\left(\mathrm{cm}^{3}\right)$ & 23.4 & 19.9 & 19.5 & 25.6 & 22.1 & 19.6 & 46.6 & 23.5 & 38.4 \\
\hline & AR & 1.3 & 1.5 & 1.5 & 2.4 & 0.7 & 1.8 & 3.1 & 1.2 & 1.8 \\
\hline \multirow{4}{*}{ 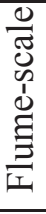 } & $\mathrm{A}_{\mathrm{W}}\left(\mathrm{cm}^{2}\right)$ & 42.7 & 38.7 & 38.5 & 36.3 & 54.3 & 34.6 & 40.3 & 44.1 & 46.3 \\
\hline & $\mathrm{A}_{\mathrm{s}}\left(\mathrm{cm}^{2}\right)$ & 92.0 & 83.9 & 83.5 & 79.2 & 115.8 & 75.1 & 87.1 & 94.9 & 99.5 \\
\hline & $\mathrm{V}\left(\mathrm{cm}^{3}\right)$ & 15.5 & 12.7 & 12.6 & 10.9 & 22.6 & 11.6 & 13.9 & 17.3 & 17.4 \\
\hline & $\mathrm{AR}$ & 6.5 & 7.1 & 7.2 & 7.6 & 5.1 & 8.0 & 6.8 & 6.3 & 5.9 \\
\hline \multirow{4}{*}{ 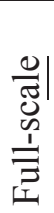 } & $\mathrm{A}_{\mathrm{w}}\left(\mathrm{cm}^{2}\right)$ & 100.5 & 90.9 & 90.4 & 85.3 & 127.6 & 81.0 & 94.7 & 103.9 & 109.1 \\
\hline & $\mathrm{A}_{\mathrm{s}}\left(\mathrm{cm}^{2}\right)$ & 216.0 & 197.1 & 196.1 & 186.0 & 271.9 & 176.2 & 204.6 & 222.9 & 233.7 \\
\hline & $\mathrm{V}\left(\mathrm{cm}^{3}\right)$ & 55.6 & 45.8 & 45.5 & 39.0 & 81.5 & 41.7 & 49.9 & 62.2 & 62.8 \\
\hline & $\mathrm{AR}$ & 3.1 & 3.5 & 3.5 & 3.7 & 2.5 & 3.9 & 3.3 & 3.0 & 2.9 \\
\hline
\end{tabular}

\subsection{Preprocessing}

\subsubsection{GEOMETRY}

The SLDPRT file of the fin was imported directly into the program Siemen's NX 9.0 for preprocessing before entering the simulation solver. The Cartesian coordinate system was used to specify the X-directional mean flow, the perpendicular to wake y-direction, and the gravitational Z-direction. The 3D single fin was enclosed in a $1.2 \times 0.4 \times 0.4 \mathrm{~m}$ rectangular box, which represents the fluid domain. The boundary box was sketched at the base of the fin in order to position the fin centrally in the Y-direction and one-quarter of the X-direction length. Figure 100 in Appendix B is an orthographic projection of the fin placement and box dimensions. It shows the fin was placed $300 \mathrm{~mm}$ from the inlet and $200 \mathrm{~mm}$ from the sidewall boundary. The box was extruded in the Z-direction $400 \mathrm{~mm}$ and had a length in the $\mathrm{X}$-direction of $1200 \mathrm{~mm}$. The gravitational force $g$ was located in the $-\mathrm{Z}$ direction; this force was set at $g=9810 \mathrm{~mm} / \mathrm{s}^{2}$.

A dynamic datum coordinate system was associated with the fins base leading edge. A datum plane was set to split the symmetric face in order to move the fins angle of attack for each simulation. The fin position changes at the leading edge of the fin base. For each 
simulation, the angle of attack changes. These values range from $0^{\circ}$ to $45^{\circ}$ in $5^{\circ}$ increments. The datum plane was also used to apply a composite curve for more accurate meshing.

FEM, SIM, and iPRT files were then associated to the PRT file in order to define flow conditions, as well as fin and wall roughness values.

\subsubsection{MESHING}

The FEM file contained the meshing information for the fin and flow boundary. To mesh each solid fin and fluid domain, the 3D tetrahedral meshing option was applied. The element shape selected was the TET10 because the elevated quantity of nodes allowed for more precise meshing across the fins curved surface. The element size chosen was $2 \mathrm{~mm}$ (Gudimetla, Kelson, \& El-Atm, 2009). Figure 11 provides an example of the blue shark (BS) fin meshing. The number of elements for this fin generated 77,321 TET10 elements. The number of elements of

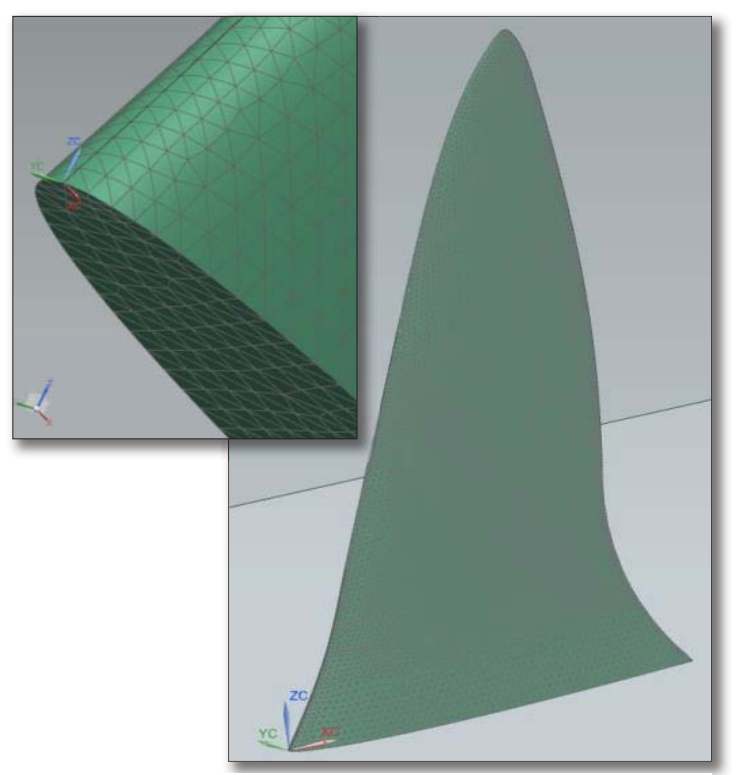

Figure 11: Meshing of a Blue Shark fin (Image by author, 2015) the nine fins averaged between $3.9 \times 10^{4}$ to $8.0 \times 10^{4}$ elements. Each fin had to be re-meshed for every change in angle of attack.

The mesh collector contained information on the type of substance and the material of the mesh. A pure substance Acrylonitrile butadiene styrene (ABS) polymer material was associated to the fin $3 \mathrm{D}$ meshing. The plastic material description included a density value of $1.05 \mathrm{e}-006 \mathrm{~kg} / \mathrm{mm}^{3}$. The Young's Modulus, E, value and the Poisson's ratio for ABS were $2 \mathrm{GPa}$ and 0.4 , respectively.

To find the practical fluid domain meshing size, a refinement meshing analysis was completed. Lift and drag force values were assessed by changing the relative element sizes. The Fixed Turbulent Viscosity turbulence model was employed to find the most accurate 
lift and drag values. The relative element size range tested, started from 0.06 to 0.001 . It was found that there were insignificant changes in the lift and drag values beyond the relative element size 0.0045 , or $5.4 \mathrm{~mm}$. Grid sizes smaller than this revealed less than a $0.05 \%$ change for the coefficients.

Water was selected as the pure substance in the flow boundary. Pure water was chosen to replicate fresh water conditions.

\subsubsection{MODELING METHOD}

The solver used to produce each simulation was the NX Thermal/Flow solver within the Flow environment. The FLOW simulator was designated as the solution modeling method in order to assess the fluid patterns around each fin and position. The k-epsilon turbulence model was chosen within the solve options by using a serial flow solver. The solution type applied was steady state. For each of the ten fins, ten angles of attack were changed to

produce 100 simulations. The fluid domain had inlet and outlet apertures to allow water to pass over the fin. The base of the fin was placed on the wall of the flow boundary to simulate a surfboard. Associated velocities, temperatures, pressures, surface roughness values, and fluid characteristics were defined within this SIM file.

\subsubsection{BOUNDARY CONDITIONS}

The mode of magnitude at the inlet was a velocity of $0.5 \mathrm{mps}$. This value was the highest velocity the laboratory flume could achieve due to pump restrictions and without extreme turbulence. The flow direction from the inlet was normal to the defined face, without swirling. The fluid distribution over the entire inlet face was set to uniform flow. External conditions included ambient temperature and humidity values. Automatic turbulence characteristics were defined so the model could calculate turbulence values during the simulation solver. Figure 12 shows an image of the fin with the enclosed flow boundary and selected boundary conditions. 
An opening condition was fixed for the outlet surface. This surface was $1.2 \mathrm{~m}$ in distance, parallel from the inlet surface.

External conditions included ambient temperature,

$\begin{array}{ll}\text { humidity, } & \text { and } \\ \text { turbulence. } & \text { The angle of attack (Image by author, 2015) }\end{array}$

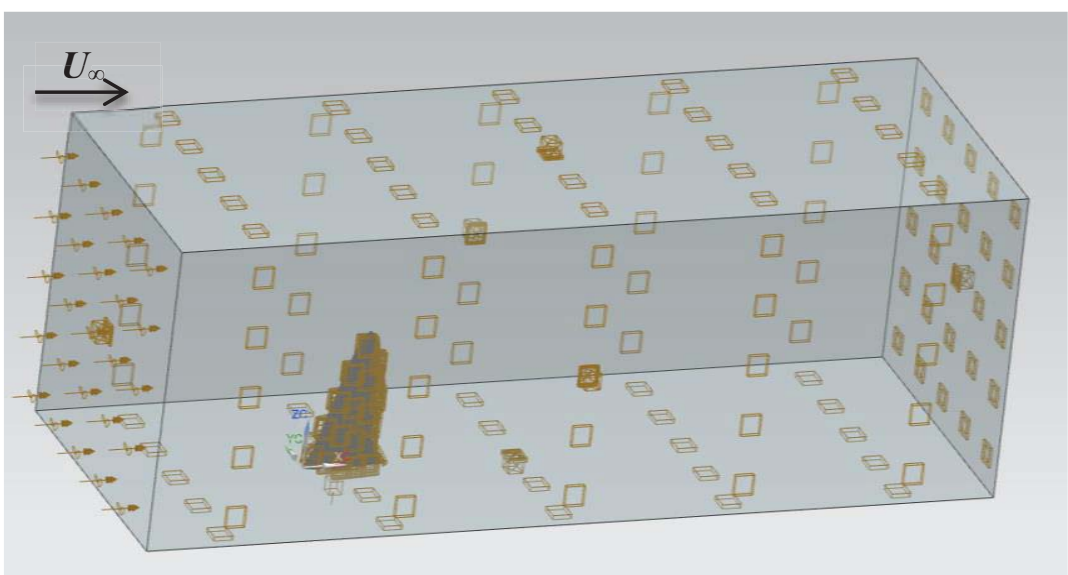

external absolute

pressure was also ambient. The flow direction was normal to the designated boundary face with uniform distribution.

The fluid domain region selected was the rectangular 3D box. The interior region was meshed through with a mesh density of $5.4 \mathrm{~mm}$ absolute element size. The material type chosen was a pure substance, setting water as the fluid material. As the pure water temperature was set to $20^{\circ} \mathrm{C}$, shown in Table 2, the ambient conditions were defined. This temperature was chosen based on average readings taken from the water channel in the laboratory. Using these analytical conditions, the Reynolds number at the base of each fin $(112 \mathrm{~mm})$ was calculated to be $5.57 \times 10^{4}$.

Table 2: Ambient Conditions of Water (Kundu \& Cohen, 2008)

\begin{tabular}{|l|l|}
\hline$\rho\left(\mathrm{kg} / \mathrm{m}^{3}\right)$ & 997 \\
\hline $\mathrm{T}\left({ }^{\circ} \mathrm{C}\right)$ & 20 \\
\hline$\mu(\mathrm{kg} / \mathrm{m} \cdot \mathrm{s})$ & $1.002 \mathrm{E}-03$ \\
\hline$\nu\left(\mathrm{m}^{2} / \mathrm{s}\right)$ & $1.004 \mathrm{E}-06$ \\
\hline $\mathrm{P}(\mathrm{MPa})$ & 0.1014 \\
\hline $\mathrm{g}\left(\mathrm{m} / \mathrm{s}^{2}\right)$ & 9.81 \\
\hline
\end{tabular}


Within the fluid domain, the fin was to be considered a flow blockage. This flow blockage had a wall friction roughness height of 1.5 microns. This was considered the fin surface boundary condition. The wall function was selected because this will model the near-wall region.

The rectangular boundary conditions were set to a free slip condition. This meant the boundary layer effect on viscous fluid state was to be neglected. A no-slip condition allowed the model to replicate a smooth surfboard in open water.

\subsection{TESTING}

\subsubsection{SIMULATION SOLVER}

A new solution solver was produced for each angle of attack, for all ten fins. The solvers

contained simulation object information including: inlet and outlet velocities, flow surface conditions, and fluid domain criteria. Reports were also setup so the model would solve for lift and drag, pressure, and flow field data. Each solution took approximately two to three hours to converge and solve.

\subsubsection{DATA ANALYSIS}

Once solved, the lift and drag forces were compared between each fin type for ten angles of attack. The solution provided lift and drag values for each angle of attack. The force data was obtained from each "Group Report", which was produced from each simulation solution. Common trends and outliers were discussed in order to understand the results. Conclusions were drawn from the comparisons between each fin from the CFD solver outputs.

The lift and drag force values were then used to calculate the associated coefficients of lift and drag. The reference area was defined by the wing area $A_{w}$ of the scaled full-sized fins; values provided in Table 1. This was the projected area of the planform view for each fin. The lift and drag values yielded units in milliNewtons, but were converted to units of Newtons in order to calculate the coefficients. These dimensionless values were used to compare to the experimental data collected in the laboratory in order to find an optimum fin. 


\subsection{OPTIMUM FIN}

\subsubsection{PREPROCESSING}

Within the NX Thermal/Flow solver program, the optimum fin was investigated at higher velocities. Typical surfing velocities, in correspondence to the suggested velocities by (Hendricks, Surfboard Hydrodynamics, Part IV: Speed, 1969) and (Edge, 2001), were chosen at 3, 5, and 7mps (Lavery, Foster, Carswell, \& Brown, 2009). Based on these upstream velocities and the fin chord length, estimates of the Reynolds number were between $3.34 \times 10^{5}$ and $7.80 \times 10^{5}$. Incident angles were chosen between $0^{\circ}$ and $25^{\circ}$, in $5^{\circ}$ increments. A new solution solver was produced for each simulation. A total of 18 simulations were run, each with a solving time of approximately two to three hours.

All variables and boundary conditions were kept the same as the initial CFD experiments, including meshing sizes and material types. The only variable altered for this portion of the project was the inlet velocity values.

\subsubsection{DATA ANALYSIS}

The output lift and drag values, from the high-velocity cases, were plotted and assessed. The results were also compared by lift and drag coefficient values. The purpose of this was to see if there were any changes in the data at higher velocities. Due to limitations in the laboratory, high-velocity experimental results were not produced for evaluation and comparison.

\subsubsection{VISUAL FLOW ANALYSIS}

The flow fields were also investigated at $0.5 \mathrm{mps}$ and the three high-velocity results. For the optimum fin, the visual results from the $0.5 \mathrm{mps}$ experiments were used to compare to between each angle of attack and to the experimental visual flow field results. Color specified velocity magnitudes and velocity vectors were applied for the CFD visual analysis of the optimum fin and all ten angles of attack. Images of the plan views and profile views, at the five sections on the fin, were used for this comparison. The five sections included the optimum fin's compression and suction sides at the base and midsection, as well as the fin tip. The degree at which flow separations initiated was 
investigated, including trailing wake motions and patterns. Velocity and pressure extremes were also located for comparison.

The three high-velocity cases were used to compare at each angle of attack and to the low velocity results. The same image sections on the fin were also used to look at the velocity magnitudes and flow patterns.

\subsection{LIMITATIONS}

There were three major limiting factors in the CFD portion of this thesis: meshing size, turbulence intensity, and the absence of a surfboard. Although, the refinement meshing study showed less than a $0.05 \%$ change in the coefficient values at finer meshing settings, the small difference does provide a minute sliver of error. The refinement meshing results are presented in Table 15 in Appendix B.

It is uncertain whether or not the applied $4 \%$ turbulence intensity was too conservative for the experimental comparisons. The proper equipment and time were major constraints concerning the turbulence intensity within the water channel.

The absence of a surfboard in the CFD method could provide conflicting flow field results when compared to the laboratory results. The surfboard lift and drag values were subtracted from the collected data in the lab, but the fin to surfboard flow interaction could alter the overall results. Once again, a more advanced setup was limited due to time and cost restraints. This thesis focuses on the flow field, as well as lifting and drag values, of the nine designed fins. The surfboard and fin interaction is outside the spectrum of the study. 


\subsection{CFD RESULTS}

This section discusses the results gathered to date. Lift and drag values are compared in order to find one optimum fin. The criterion is based on values of highest lift and lowest drag. Table 3 provides a list of all nine species and their associated acronym codes used in this report.

Table 3: Species name and acronym code

\begin{tabular}{|l|l|}
\hline Species & Code \\
\hline Blue Shark & BS \\
Pacific Spotted Dolphin & SD \\
Fin Whale & FW \\
Swordfish & SF \\
Short-finned Pilot Whale & SFPW \\
Marlin & M \\
Killer Whale & O \\
Dall's Porpoise & DP \\
Shortfin Mako & SFM \\
\hline
\end{tabular}

\subsubsection{LIFT FORCE DATA}

There was a consistent trend in the results of the lift data. Figure 13 shows the lift curve data at all angles of attack for each fin type. The shapes of the lift curves were anticipated, as it was a direct result of the airfoil theory (Gudimetla, Kelson, \& El-Atm, 2009). At small angles of attack, the lift forces increased in a linear fashion with the angle of attack until a maximum lift value was reached. All nine fins had peak lift values at $25^{\circ}$. This angle was considered the critical angle of attack. Lifting value growth was demonstrated from zero degrees to $25^{\circ}$ angle of attack. From $25^{\circ}$ to $45^{\circ}$ angle of attack, the lifting values declined due to hydrofoil stall. 


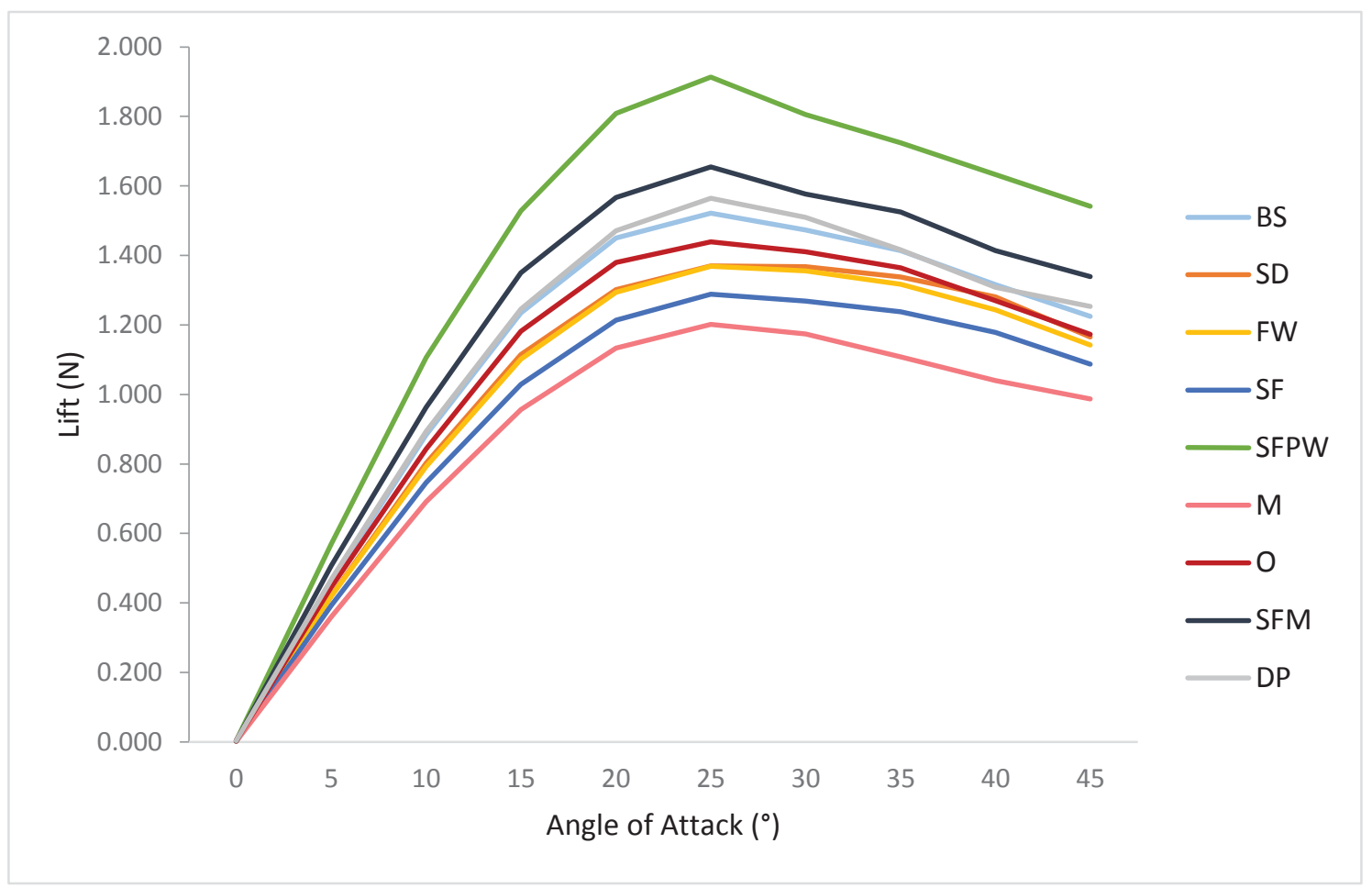

Figure 13: Lift (N) from CFD data collected for nine fins at ten angles of attack (0$\left.45^{\circ}\right), \mathrm{U}_{\infty}=0.5 \mathrm{mps}$

There was a correlation between surface area and lift values. The quantified lift value data is presented in Table 4 and surface area values for each fin are provided in Table 1. At higher surface area measurements, lifting values were greater. The Short-finned Pilot Whale (SFPW) had the highest lift values for all angles of attack. This fin also had the most surface area of all nine fins. Conversely, the Marlin (M) had the smallest surface area of all the fins with the lowest lift values at all angles of attack.

At the critical angle of attack, lift values ranged from $1.20 \mathrm{~N}$ for the $\mathrm{M}$ to $1.91 \mathrm{~N}$ for the SFPW. There was a $45.7 \%$ difference in maximum lift between the SFPW and the Marlin.

The trends of the nine fins were similar, but have different magnitudes of lift curvature. Focusing primarily on $25^{\circ}$, the order of lift values followed the order of surface area magnitudes. The Shortfin Mako shark (SFM) was the fin with the next highest lift force, reaching a lift value $1.81 \mathrm{~N}$ at $25^{\circ}$. The Dall's Porpoise (DP) and the Blue Shark (BS) fins provided similar lifting curve shapes. The DP fin had higher lift values compared to the BS 
from $0^{\circ}$ to $35^{\circ}$. At $40^{\circ}$ to $45^{\circ}$ the $\mathrm{BS}$ surpassed the DP with lifting values of $1.32 \mathrm{~N}$ and $1.22 \mathrm{~N}$, respectively. The Orca $(\mathrm{O})$ produced a lift force of $1.44 \mathrm{~N}$ at the $25^{\circ}$ critical angle of attack. The Fin Whale (FW) and the Spotted Dolphin (SD) also provided lift curves that resembled the same shape, with many values numerically comparable at several angles of attack. Specifically at $5^{\circ}$ and $25^{\circ}$, the FW and SD had respective lift values of $0.42 \mathrm{~N}$ and $1.37 \mathrm{~N}$. The Swordfish (SF) was the second to lowest fin, with a lift value of $1.29 \mathrm{~N}$ at $25^{\circ}$.

Table 4: Lift $(\mathrm{N})$ from CFD data collected for nine fins at ten angles of attack $\left(0-45^{\circ}\right)$, $\mathrm{U}_{\infty}=0.5 \mathrm{mps}$

\begin{tabular}{|l|lllllllll|}
\hline $\boldsymbol{\alpha}\left({ }^{(}\right)$ & BS & SD & FW & SF & SFPW & M & O & DP & SFM \\
\hline 0 & 0.00 & 0.00 & 0.00 & 0.00 & 0.00 & 0.00 & 0.00 & 0.00 & 0.00 \\
5 & 0.46 & 0.42 & 0.42 & 0.39 & 0.57 & 0.36 & 0.45 & 0.47 & 0.51 \\
10 & 0.88 & 0.80 & 0.79 & 0.75 & 1.11 & 0.69 & 0.84 & 0.89 & 0.96 \\
15 & 1.24 & 1.12 & 1.10 & 1.03 & 1.53 & 0.96 & 1.18 & 1.25 & 1.35 \\
20 & 1.45 & 1.30 & 1.29 & 1.21 & 1.81 & 1.13 & 1.38 & 1.47 & 1.57 \\
25 & 1.52 & 1.37 & 1.37 & 1.29 & 1.91 & 1.20 & 1.44 & 1.56 & 1.66 \\
30 & 1.47 & 1.37 & 1.36 & 1.27 & 1.81 & 1.17 & 1.41 & 1.51 & 1.58 \\
35 & 1.41 & 1.34 & 1.32 & 1.24 & 1.72 & 1.11 & 1.36 & 1.42 & 1.52 \\
40 & 1.32 & 1.28 & 1.24 & 1.18 & 1.63 & 1.04 & 1.27 & 1.31 & 1.41 \\
45 & 1.22 & 1.17 & 1.14 & 1.09 & 1.54 & 0.99 & 1.17 & 1.25 & 1.34 \\
\hline
\end{tabular}

Note: yellow highlighted quantities represent the highest L/D ratios and blue highlighted quantities represented the lowest $\mathrm{L} / \mathrm{D}$ ratios

\subsubsection{DRAG FORCE DATA}

A consistent trend also occurred in the results of the drag data. Figure 14 shows the drag curve data at all ten angles of attack for each of the nine fin types. As expected, the drag values increased as the fins' angle of attack moved from zero to $45^{\circ}$. The drag values were greatest at $45^{\circ}$ for all nine fins.

Once again, there was a correlation between the drag values and surface area. Fins with higher surface area showed higher drag values. Table 5 provides raw drag value data for each of the nine fins at all ten angles of attack. Having the largest surface area, the SFPW provided the highest drag values for each angle of attack. At $25^{\circ}$, this fin's drag value was $0.85 \mathrm{~N}$ and $1.60 \mathrm{~N}$ at $45^{\circ}$. 


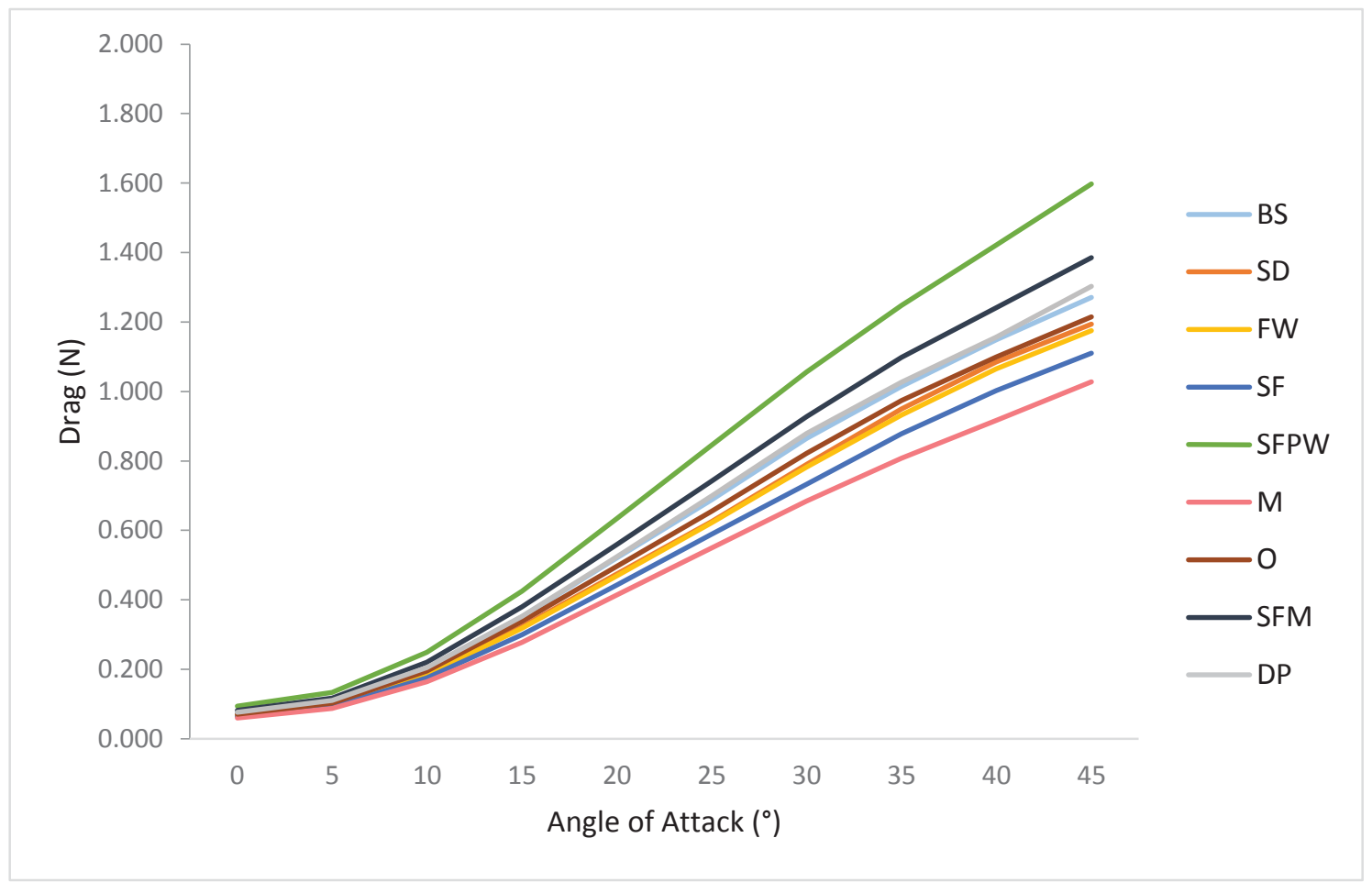

Figure 14: Drag $(\mathrm{N})$ from CFD data collected for nine fins at ten angles of attack (0$\left.45^{\circ}\right), \mathrm{U}_{\infty}=0.5 \mathrm{mps}$

Table 5: Drag (N) from CFD data collected for nine fins at ten angles of attack $\left(0-45^{\circ}\right)$, $\mathrm{U}_{\infty}=0.5 \mathrm{mps}$

\begin{tabular}{|l|lllllllll|}
\hline $\boldsymbol{\alpha}\left(^{\circ}\right)$ & BS & SD & FW & SF & SFPW & M & O & DP & SFM \\
\hline 0 & 0.07 & 0.07 & 0.07 & 0.06 & 0.09 & 0.06 & 0.07 & 0.08 & 0.08 \\
5 & 0.11 & 0.10 & 0.10 & 0.09 & 0.13 & 0.09 & 0.10 & 0.11 & 0.12 \\
10 & 0.20 & 0.19 & 0.18 & 0.18 & 0.25 & 0.16 & 0.20 & 0.20 & 0.22 \\
15 & 0.35 & 0.32 & 0.32 & 0.30 & 0.43 & 0.28 & 0.34 & 0.35 & 0.38 \\
20 & 0.52 & 0.47 & 0.47 & 0.44 & 0.63 & 0.41 & 0.50 & 0.52 & 0.56 \\
25 & 0.69 & 0.63 & 0.62 & 0.59 & 0.85 & 0.55 & 0.66 & 0.70 & 0.74 \\
30 & 0.86 & 0.79 & 0.78 & 0.73 & 1.06 & 0.68 & 0.82 & 0.88 & 0.93 \\
35 & 1.01 & 0.95 & 0.93 & 0.88 & 1.25 & 0.81 & 0.97 & 1.03 & 1.10 \\
40 & 1.15 & 1.08 & 1.06 & 1.00 & 1.42 & 0.92 & 1.10 & 1.16 & 1.24 \\
45 & 1.27 & 1.19 & 1.18 & 1.11 & 1.60 & 1.03 & 1.21 & 1.30 & 1.39 \\
\hline
\end{tabular}

Note: yellow highlighted quantities represent the highest L/D ratios and blue highlighted quantities represented the lowest L/D ratios 
The SFM was again the second leading fin, with drag forces reaching $0.74 \mathrm{~N}$ at $25^{\circ}$ and $1.39 \mathrm{~N}$ at $45^{\circ}$. The DP and the BS fins had similar curve shapes due to equivalent drag values from $5^{\circ}$ to $20^{\circ}$. From $25^{\circ}$ to $45^{\circ}$, the DP fin provided slightly higher drag quantities compared to the BS. The drag measurements for the FW and the SD were extremely close in value, which provided almost identical drag curves. At angles greater than $20^{\circ}$, the DP produced slightly higher drag forces. The SF was the second most efficient fin in terms of least amount of drag, with a drag force of $0.62 \mathrm{~N}$ at $25^{\circ}$ and $1.18 \mathrm{~N}$ at $45^{\circ}$. The Marlin (M) had the smallest surface area of all the fins, which led to the lowest drag forces at all angles of attack. At $25^{\circ}$ the measured drag force was $0.55 \mathrm{~N}$ and at $45^{\circ}$ the drag force was $1.03 \mathrm{~N}$.

\subsubsection{LIFT-TO-DRAG RATIO}

The lift-to-drag ratio (L/D) provided a basis in deciding which fin was the most efficient. Efficiency values were greatest when fins produced the high lift values and low drag values. The fin(s) with higher L/D ratios were considered more efficent than compared to fins with low $\mathrm{L} / \mathrm{D}$ values. The fin with the highest efficiencies at the most angles of attack, comapred to the other eight fins, would be considered the optimum fin. It was not expected that one fin would provide the highest $\mathrm{L} / \mathrm{D}$ ratios at all ten angles of attack.

Figure 15 provides calculated L/D ratios from collected CFD data for each of the nine fins. The shapes of the curves were expected, as these were typical L/D ratio trends for symmetric hydrofoil 3D finite wings (fins). The curves were also clustered because the fins were closely related in value for each angle of attack, as shown in Table 6. There did not seem to be an obvious common trend within the $\mathrm{L} / \mathrm{D}$ ratio data. This meant one fin did not consistently show high efficiency values for all angles of attack and one fin did not consistently show low efficiency values for all angles of attack. For each angle, the yellow highlighted quantities represented the highest $\mathrm{L} / \mathrm{D}$ ratios and the blue highlighted quantities represented the lowest $\mathrm{L} / \mathrm{D}$ ratios. The $\mathrm{SD}, \mathrm{FW}$, and $\mathrm{SF}$ each peaked at $5^{\circ}$ angle of attack, while the remaining fins peaked at $10^{\circ}$. The Marlin fin had the most commonly occurring low efficiency measurements. Out of the ten angles of attack, the Marlin was the least efficient fin for six angles. The SFM shark was the most efficient fin at $5^{\circ}$, but had the lowest $\mathrm{L} / \mathrm{D}$ ratio at $30^{\circ}$. This also occurred for the FW and SF. The FW was the least 
efficient at $0^{\circ}$, but most efficient at $30^{\circ}$ and $35^{\circ}$. The SF had the lowest ratio at $15^{\circ}$, but the highest at $45^{\circ}$.

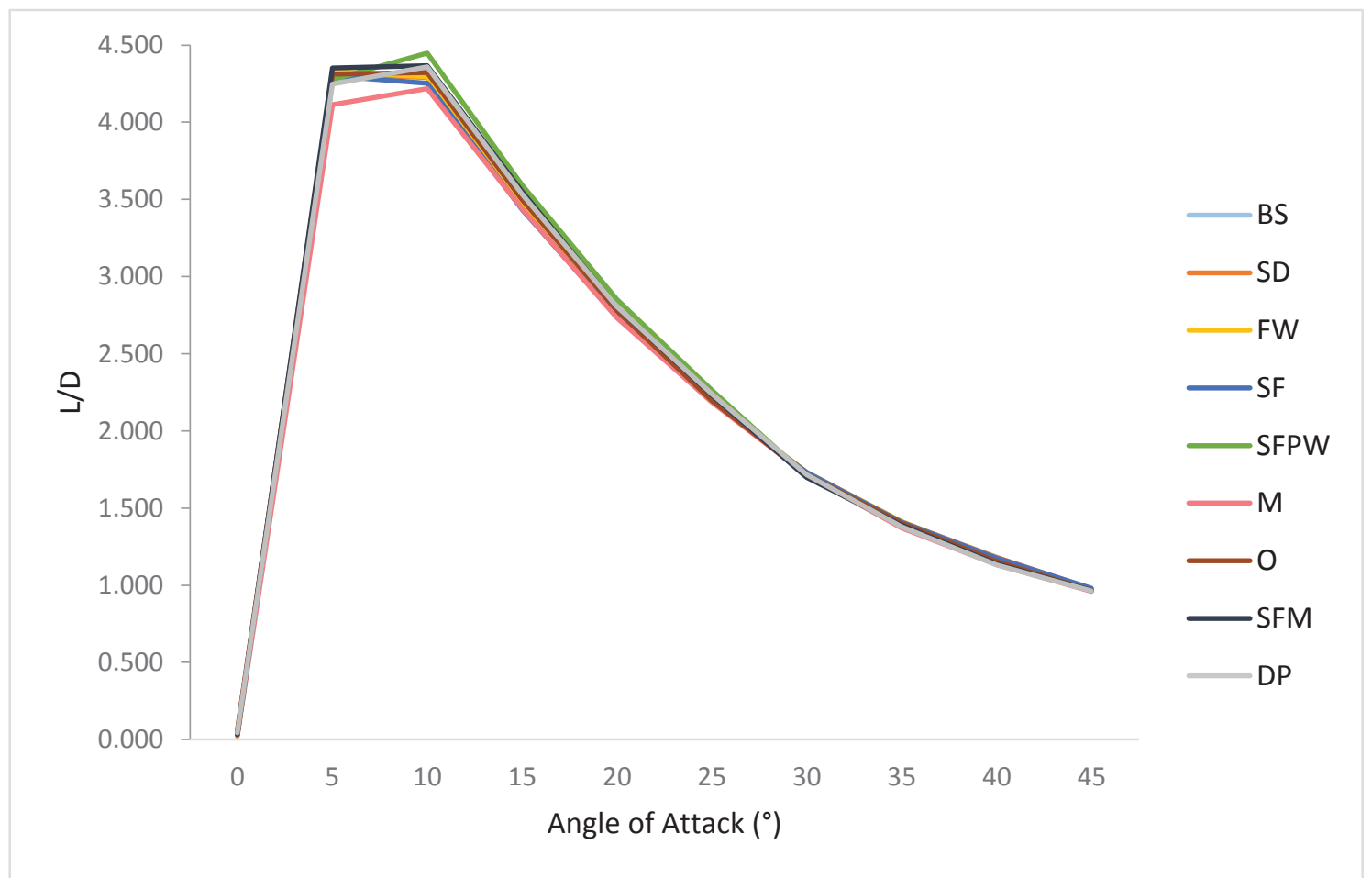

Figure 15: Lift to drag ratio values from CFD data collected for nine fins at ten angles of attack $\left(0-45^{\circ}\right), \mathrm{U}_{\infty}=0.5 \mathrm{mps}$

Table 6: Lift to Drag Ratio (L/D) from CFD data collected for nine fins at ten angles of attack $\left(0-45^{\circ}\right), \mathrm{U}_{\infty}=0.5 \mathrm{mps}$

\begin{tabular}{|l|lllllllll|}
\hline $\boldsymbol{\alpha}\left({ }^{\circ}\right)$ & BS & SD & FW & SF & SFPW & M & O & DP & SFM \\
\hline 0 & 0.02 & 0.05 & 0.02 & 0.04 & 0.04 & 0.02 & 0.06 & 0.04 & 0.03 \\
5 & 4.27 & 4.31 & 4.33 & 4.30 & 4.28 & 4.11 & 4.31 & 4.25 & 4.35 \\
10 & 4.33 & 4.29 & 4.30 & 4.25 & 4.45 & 4.22 & 4.32 & 4.36 & 4.37 \\
15 & 3.51 & 3.45 & 3.46 & 3.43 & 3.59 & 3.44 & 3.50 & 3.53 & 3.55 \\
20 & 2.78 & 2.75 & 2.76 & 2.74 & 2.85 & 2.73 & 2.78 & 2.81 & 2.80 \\
25 & 2.21 & 2.19 & 2.20 & 2.19 & 2.26 & 2.19 & 2.20 & 2.24 & 2.23 \\
30 & 1.70 & 1.73 & 1.73 & 1.73 & 1.71 & 1.71 & 1.72 & 1.72 & 1.70 \\
35 & 1.39 & 1.41 & 1.41 & 1.41 & 1.38 & 1.37 & 1.40 & 1.38 & 1.39 \\
40 & 1.15 & 1.18 & 1.17 & 1.18 & 1.15 & 1.13 & 1.15 & 1.13 & 1.14 \\
45 & 0.96 & 0.98 & 0.97 & 0.98 & 0.97 & 0.96 & 0.97 & 0.96 & 0.97 \\
\hline
\end{tabular}

Note: yellow highlighted quantities represent the highest L/D ratios and blue highlighted quantities represented the lowest L/D ratios 
At $10^{\circ}$, the SFPW had the peak $\mathrm{L} / \mathrm{D}$ ratio value (4.45) among all the fins for the ten angles of attack. Between $10^{\circ}$ and $25^{\circ}$, this fin had the greatest $\mathrm{L} / \mathrm{D}$ ratios of the nine fins. It had the most commonly occurring maximum L/D ratio measurements; four out of the ten angles, the SFPW was the most efficient fin. The FW had the greatest $\mathrm{L} / \mathrm{D}$ ratios at $30^{\circ}$ (1.73) and $35^{\circ}(1.41)$, but had the lowest efficiency of all nine fins at $0^{\circ}(0.02)$. The SD produced the highest $\mathrm{L} / \mathrm{D}$ ratio at $40^{\circ}(1.18)$, while the $\mathrm{SF}$ was the most efficient fin at $45^{\circ}$ (0.98). The Marlin was almost consistently the least efficient fin for the CFD L/D analysis.

\subsection{DISCUSSION}

The results of this CFD portion of the study followed typical lift and drag curves according to the airfoil theory and classical wing theory (Kundu \& Cohen, 2008). Lift forces increased as the angle of attack was amplified. Lift forces peaked at a critical angle of attack and stall occurred after that angle. Drag forces increased as the angle of attack moved from $0^{\circ}$ to $45^{\circ}$.

Based on the aspect-ratios for each fin, found in Table 1, it was expected that the Marlin and SF would generate greater lift forces and lower drag forces than the other fins. This was because these two fins had the highest AR values. It is thought that wings (or fins) with high aspect ratios would have lower drag and slightly higher lift than fins with lower aspect ratio values (NASA, 2014). High AR wings are typically long and thin, where low AR wings are shorter (NASA, 2014). The problem with comparing AR for this problem was the base chord length and fin wing span were identical for all nine fins. The only changing dimension between each fin was the surface area size.

There was a relationship between the surface area measurements of the fin and lift forces; this was also true for the drag forces. The FW and the SD were quantitatively close in value, which produced near identical associated lift and drag curves. Referring back to Table 1, the wing area $\mathrm{A}_{\mathrm{w}}$ of the SD was only $0.54 \%$ larger than the area of the SF. This could give some validation as to why the fins shared such similarities in both lift and drag information. 
The SFPW provided the highest lift and drag values at all angles of attack. This was due to its larger surface area in comparison to the other studied fins. The SFPW was more efficient than the other nine fins at smaller angles of attack. It was the most efficient fin between the angles of $10^{\circ}$ and $25^{\circ}$ and had a peak L/D ratio at $10^{\circ}$. According to Lavery et al. (2009), these forces would be most effective up to $10^{\circ}$ to $12^{\circ}$ angle of attack. For a single fin at the lower ranges of incident angles, higher $\mathrm{L} / \mathrm{D}$ ratios improve turning and speed of the surfboard setup (Carswell \& Lavery, 2006). For typical surfing conditions, the SFPW would offer lift values for easy maneuvering and the large surface area provides greater stability benefits. At higher angles of attack, the SD, FW, and SF yielded maximum efficiency values. There was only about a 3\% difference between those maximum L/D ratios and the associated L/D ratios from the SFPW. This variance is likely not significant in the overall performance of the surfboard and fin setup.

The Marlin had the lowest lift and drag forces of all nine fins. There was a $45.7 \%$ difference in the maximum lift between the SFPW and the Marlin, which could provide noticeable impacts on the overall efficiency and performance of the surfboard setup. The smaller lift and drag values at low incident angles improve the directional stability and speed of the surfboard setup (Gudimetla, Kelson, \& El-Atm, 2009). When surfing larger waves, less manuevering is required, but greater amounts of stabability are necessary (Gudimetla, Kelson, \& El-Atm, 2009). Because of this, the low L/D ratios make the Marlin more suitable for big-wave surfing.

It was unknown whether or not the sweep angle of each fin caused variation in the data. The diversity of this angle could have improved performance for some fins, but it was unclear if a greater degree of sweep was beneficial. This fin dimension can change the surfer's ability to pivot. The focuses of this thesis were to measure and compare each fin's lift and drag forces, as well as investigate the flow field for one fin at different angles of attack. Although the sweep angles could have distorted the outcomes of the results, the effect of sweep was outside the scope of this thesis.

All methods of modeling have some degree of error. A source of random of error in this CFD model could include the turbulence intensity set for the flow boundary. Typical 
surfing wave turbulence intensities were unknown. Because of this, automatic turbulence characteristics were defined so the model could calculate turbulence values during the simulation solver. Turbulence in real surfing conditions may be higher or lower than these input values. The k-epsilon two-equation model, to solve for turbulence, also has some degree of error. Due to time and space restrictions, a turbulence model with a higher level of accuracy was not used.

Velocity values were set to $0.5 \mathrm{mps}$, which were not up to typical surfing wave velocities. Although the lift and drag coefficients could be used to find these higher velocity forces, slight changes in the data could have occurred due to the low velocity input values. For future experimentation in this field, a larger water channel to support high velocities with a wave producer should be built. Teaming up with another university or a surfboard fin design company could help improve the results and methods for this topic.

A more accurate method of modeling such as Large Eddy Simulation (LES) or Direct Numerical Simulation (DNS) should be used. This would increase the accuracy of the boundary and flow calculations, but solving time would be extremely long and a large amount of data saving space would be required. These results should be compared to tested data in the laboratory and real surfing conditions on the lake or ocean. Results should be compared between each of the models and lab experiments to measure the accuracy and differences. 


\section{Chapter 5: Laboratory Work for Single Fin}

It was necessary to apply experimental testing to verify the computational results for understanding the fundamental hydrodynamic effects of a surfboard and the fin system. The lift and drag force measurements were taken for each of the nine fins at ten angles of attack. For the purpose of comparing CFD and experimental results, one velocity was applied to find an optimum fin. Once all lift and drag data was gathered and analyzed, one fin was chosen for further experimentation. These results and comparisons are presented within this section of the thesis report; Section 5.5 and Section 5.6.

\subsection{PREPROCESSING}

\subsubsection{SCALING SURFBOARD AND FINS}

To avoid any disruption from the wall and floor boundaries, the surfboard and fins were scaled down to fit the water channel. These calculations were done in Microsoft Excel. The width of the surfboard was scaled to one-third of the width of the water channel in order to avoid velocity disturbances between the surfboard and channel walls. All other dimensions were scaled down proportionally. The length and width of the board was found to be $1.59 \mathrm{~m}$ and $0.31 \mathrm{~m}$, respectively. Based on the scaled surfboard width dimension, each fin was also proportionally measured down. The fin base widths and heights were calculated to $73.09 \mathrm{~mm}$ and $116.04 \mathrm{~mm}$, respectively; approximately 2.3 times smaller than the fullscaled fin dimensions. All dimensions were applied to the original surfboard size and fullscaled fin 3D models in Solidworks using the Scaler tool.

\subsubsection{Fin PRINTING AND FABRICATION}

The fin files were saved as STL files to be imported into Cura. Each fin was formatted for printing and uploaded to a SandDisk memory card. This allowed the Ultimaker2 to access the fin files for printing. The fins were all printed separately. Once each fin was printed, the surfaces were sanded down using a 120-grit through 1500-grit grain sand paper. Two thin layers of Sikkens Colorbuild Plus Primer were sprayed on each fin to provide a completely smooth surface and for the purpose of waterproofing. This was done for both the full-scaled fins and the flume-scaled fins. The Reynolds number based on chord length 
for each flume-scaled fin $(73.09 \mathrm{~mm})$, the free-stream velocity of $0.5 \mathrm{mps}$, viscosity of $1.004 \times 10^{-6} \mathrm{~m}^{2} / \mathrm{s}$, and water temperature at $20^{\circ} \mathrm{C}$ was calculated to be $3.64 \times 10^{4}$.

\subsubsection{SURFBOARD CONSTRUCTION}

The scaled down surfboard dimensions were applied to the construction of the surfboard in the Chopstix shop in Chassell, MI. To build the surfboard, Polystyrene foam was used as the core material. The initial foam block was cut to a length of $159.3 \mathrm{~cm}$ and a width of $30.7 \mathrm{~cm}$. Using a jigsaw, it was then cut in two halves down the center length of the block. A wooden center stringer was used to bind the two block pieces in order to strengthen the surfboard. Using a palm-grip random orbit sander, the rocker was shaped into the block. The dimensions were then measured on the block and cut using a handsaw. Several stages of sandpaper grades were used to shape the board to the exact specifications.

A 6oz. E-glass fiberglass cloth was wrapped around the surfboard and saturated in epoxy resin to provide additional strength to the surfboard. One layer of fiberglass cloth was sized to the deck of the surfboard and two layers of cloth were sized to wrap the deck and rails. Once the resin and cloth hardened, two layers of epoxy resin were coated on to provide a smooth, water-resistant layer. The ratio of two parts resin to one part hardener was mixed together to glass the surfboard. Sanding in between dried coats was completed by hand using 120 - to 800 -grit sandpaper.

\subsubsection{FIN INSERT SYSTEM}

A fin insert system was designed to improve the efficiency of this project. It was modeled in Solidworks and printed on the Ultimaker2 3D printer. The purpose of this system was to easily reposition the fin's angle of attack before each laboratory experiment. There were three parts to the system: the receiver-box, angle-control, and tab.

The $102.4 \mathrm{~cm}^{3}$ tab was designed to attach to the base of the fin and to fit into the anglecontrol. This cube had a $1.27 \mathrm{~cm}^{2}$ base dimension and $0.64 \mathrm{~cm}$ height. Epoxy resin was used as an adhesive to bind the tip of the fin to the center of the tab. This procedure was done for all nine fins. The angle-control was modeled as a solid $2.25 \mathrm{~cm}$ diameter cylinder at a $0.84 \mathrm{~cm}$ depth. An extrusion was made at the center to fit the cubed tab. The receiver-box 
was built into the surfboard bottom deck, where the single fin would be positioned. This box was designed as a $2.51 \mathrm{~cm}$ diameter cylinder. It had a height of $1.12 \mathrm{~cm}$. The center of the receiver-box was finished hallow to allow the attachment of the angle-control.

To setup this system, the angle-control part was positioned into the receiver-box at a defined angle of attack. A small single screw was drilled through the angle-controller and receiver box into the surfboard to secure the position of the modules. The adjoined fin and tab assembly were then inserted into the angle-controller. To fasten the two components together, an additional screw was drilled through the angle-control and tab.

\section{$5.2 \quad$ TESTING}

\subsubsection{EXPERIMENTAL SETUP}

The surfboard floated in the water, 5m downstream from the PVC-pipe flow straightener section. A wooden board was placed across the water channel, upstream of the measurement area. It was secured to the top of the water channel. At the center of the wooden board was an attached wooden dowel that held the force gauge. Cords were fastened from two hooks on the deck of the board to the spring gauge. The hooks were placed on opposing rail edges, at the widest section of the board. These were used to attach the spring gauges to this stationary origin and the moving surfboard. The placement of the hooks and force gauge allowed the surfboard to ride parallel to oncoming flow. There were various spring sensitivities for determining tension force of the surfboard and fin setup. As the surfboard pulled itself away from the origin, the extension of the spring was measured. This spring displacement gave value to the magnitude of force the object had at a certain water velocity and associated drag forces. The surfboard drag force was first examined as the control. All fin setup experimental drag force values were subtracted from the control value to give the drag force of the fin itself.

A second wooden board and dowel setup was placed near the tail section of the board. Attached to the deck of the board, the third hook was placed at the center of the fins base area. A cord fastened the hook to a second spring gauge. This gauge was positioned perpendicular to the flow direction to measure lift forces of the fin and surfboard. At angles 
of attack greater than zero, the tail of the surfboard pulled itself away from the spring gauge. The spring displacement gave lift force values.

\subsubsection{EXPERIMENTAL PROCEDURE}

The fin system, with the attached fin, was fastened into the surfboard. The fin was then positioned into the desired angle of attack and the screws were tightened to secure the setup. The board was lowered into the $0.5 \mathrm{mps}$ running water. Each spring gauge was lowered parallel to the board attachments. For each trial, the spring gauges were viewed for approximately one minute to obtain the lift and drag range. Once a lift and drag range was obtained, the surfboard was taken out of the water and the angle of attack was changed. For each fin, at all ten angles of attack, two trials were run. The data was logged into Microsoft Excel for further analysis.

For the first set of trial experiments, the $0-2 \mathrm{~N}$ spring gauge was used to measure drag. The $0-1 \mathrm{~N}$ spring gauge was used to measure lift. During the second trial, the devices were switched. The $0-1 \mathrm{~N}$ spring gauge was used to measure drag and the $0-2 \mathrm{~N}$ spring gauge was used to measure lift.

All spring loads were tested and calibrated before they were secured in the water channel for the experiments. To assess the level of accuracy and consistency, a Gage Repeatability \& Reproducibility (GRR) study was done (Pandiripalli, 2007). One fin at, one angle of attack, was chosen to administer the analysis: the SFPW at $15^{\circ}$ angle of attack. Minimum and maximum lift and drag values were measured and repeated 20 times. Based on the GRR decision-making criteria, acceptable levels of error were defined for the measurement system (MS): error $<10 \%=$ MS is acceptable, $10 \%<$ error $<30 \%=$ MS may be acceptable, $30 \%<$ error $=$ MS needs improvement (Pandiripalli, 2007). The average lift and drag values produced $13 \%$ and $14 \%$ error. The minimum and maximum error values were less than $30 \%$. All error readings satisfied the guidelines, so the laboratory data was considered acceptable for analysis and CFD comparisons; the repeatability test is presented in Table 16 in Appendix B. 


\subsubsection{DATA ANALYSIS}

The minimum and maximum force value range was typed into Microsoft Excel. An average was found for each angle of attack for both trials. An average was then found between the two trials for each angle of attack. Drag averages were subtracted from the measured drag of the surfboard, to provide only fin drag forces. The lift and drag force averages were plotted for all nine fins for the purpose of comparing the experimental result curves. Conclusions were made about the shapes of the curves, as well as any trends that occurred. Maximum lift and drag values were found and a critical angle of attack was identified. The quantitative data was used to provide further evidence to support the conclusions of the results.

Lift and drag coefficients were then calculated so comparisons could be made between the nine fins. Assessments were made between the CFD and laboratory results using these values, but accurate comparisons could not be made because the Reynolds numbers were not equivalent. The wing area $A_{w}$ of the scaled lab fins defined the reference area used in the $C_{L}$ and $C_{D}$ calculations; the $A_{W}$ values for each fin are provided in Table 1. An optimum fin was identified from the lift and drag results.

\section{$5.3 \quad$ OPTIMUM FIN}

\subsubsection{PREPROCESSING}

Within the NX Thermal/Flow solver program, the optimum fin was investigated at higher velocities. Typical surfing velocities, in correspondence to the suggested velocities by (Hendricks, Surfboard Hydrodynamics, Part IV: Speed, 1969) and (Edge, 2001), were chosen at 3, 5, and 7mps (Lavery, Foster, Carswell, \& Brown, 2009). Incident angles were chosen between $0^{\circ}$ and $25^{\circ}$, in $5^{\circ}$ increments. A new solution solver was produced for each simulation. A total of 18 simulations were run, each with a solving time of approximately two to three hours.

All variables and boundary conditions were kept the same as the initial CFD experiments, including meshing sizes and material types. The only variable altered for this portion of the project was the inlet velocity values. 


\subsubsection{DATA ANALYSIS}

The output lift and drag values, from the high-velocity cases, were plotted and assessed. The results were also compared by lift and drag coefficient values. The purpose of this was to see if there were any changes in the data at higher velocities. Due to limitations in the laboratory, high-velocity experimental results were not produced for evaluation and comparison.

\subsubsection{VISUAL FLOW ANALYSIS}

For the visual analysis portion of the experimentation, the surfboard and all the setup equipment was taken out of the flume. A protractor was used to draw the ten angles of attack on the wooden bed of the water channel. The base of the optimal fin was glued to the bed of the water channel at a specified angle of attack. The reason for this was to keep the fin from moving and to easily take video evidence of the flow reactions to the positioned fin. The water was refilled in order to record visual data and this was done for all ten angles of attack for the optimum fin.

At $0.5 \mathrm{mps}$, the dye was injected directly in front of the fin at five different positions along the fin's length. Injections were placed at the base and midsection of the fin, on both the compression and suction sides. The tip of the fin also injected with dye. This procedure was done twice so that the camera could capture a plan view and profile view of the flow. The profile view of the fin was studied by filming through the side window on the water channel. The plan view was filmed through a circular underwater viewer. The lens was sixinches in diameter and placed just below the water surface to film the flow field. The camera captured slow motion video at $240 \mathrm{fps}$ to understand the movement of the dye around the optimum fin. Screen shots of the video were taken in one-second intervals for image comparisons. Each video and screenshot progression was examined for the comparison of the CFD visual results.

\section{$5.4 \quad$ LIMITATIONS}

The limitations in this thesis are mainly in the experimental data collection methods. The spring gauges provide a high level of uncertainty and error. Although validations were 
done, in order to make sure the process was consistent, accuracies are still questionable. Human error can contribute to the majority of the uncertainty. Because these readings were captured on an analogue scale, some rounding error was made. The gauge reader also had to make decisions on the average range of force values the spring gauge presented, without biases. The costs and knowledge of setting up a more accurate strain gauge and recording device was costly and was outside of the realm of this thesis.

The water channel size and velocity outputs were also limiting. The pump size and upstream turbulence only allowed for a maximum of approximately $0.5 \mathrm{mps}$, dependent on water depth. This velocity is not a typical speed a surfable wave, or surfer, would produce. Because of boundary effects, the water channel dimensions limited the size of the fins and surfboard. The full size fins, which were tested in the CFD program, were not appropriate for the laboratory experiments. To fix this, the fins were scaled based on the size of the water channel. Lift and drag forces were not comparable, so lift and drag coefficients were calculated.

The material used to print the 3D fins was not typical for surfboard fins on the market today. Although the inlet velocities were fairly low, restrictions in the flexibility could change the performance significantly if used in real wave conditions.

\subsection{LABORATORY RESULTS}

This section discusses the results gathered to date. Several conclusions were drawn in attempt to understand what was occurring in the laboratory data. Lift and drag values were compared in order to find one or more optimum fin(s). The criterion was based on values of highest lift and lowest drag. The flow patterns were visually and quantitatively analyzed and reported.

\subsubsection{LIFT FORCE DATA}

The lift force results showed similar trends in the shape of the curves. Figure 16 shows the lift curve data at all angles of attack for each fin type. Many of the fins followed the typical shape of a lift force curve, for a symmetrical 3D finite wing (fin). As the angle of attack increased, lifting values increased until a maximum lift value was reached. All nine fins 
had peak lift values at $25^{\circ}$, except the BS and $\mathrm{M}$. These two fins generated a maximum lift at $20^{\circ}$.

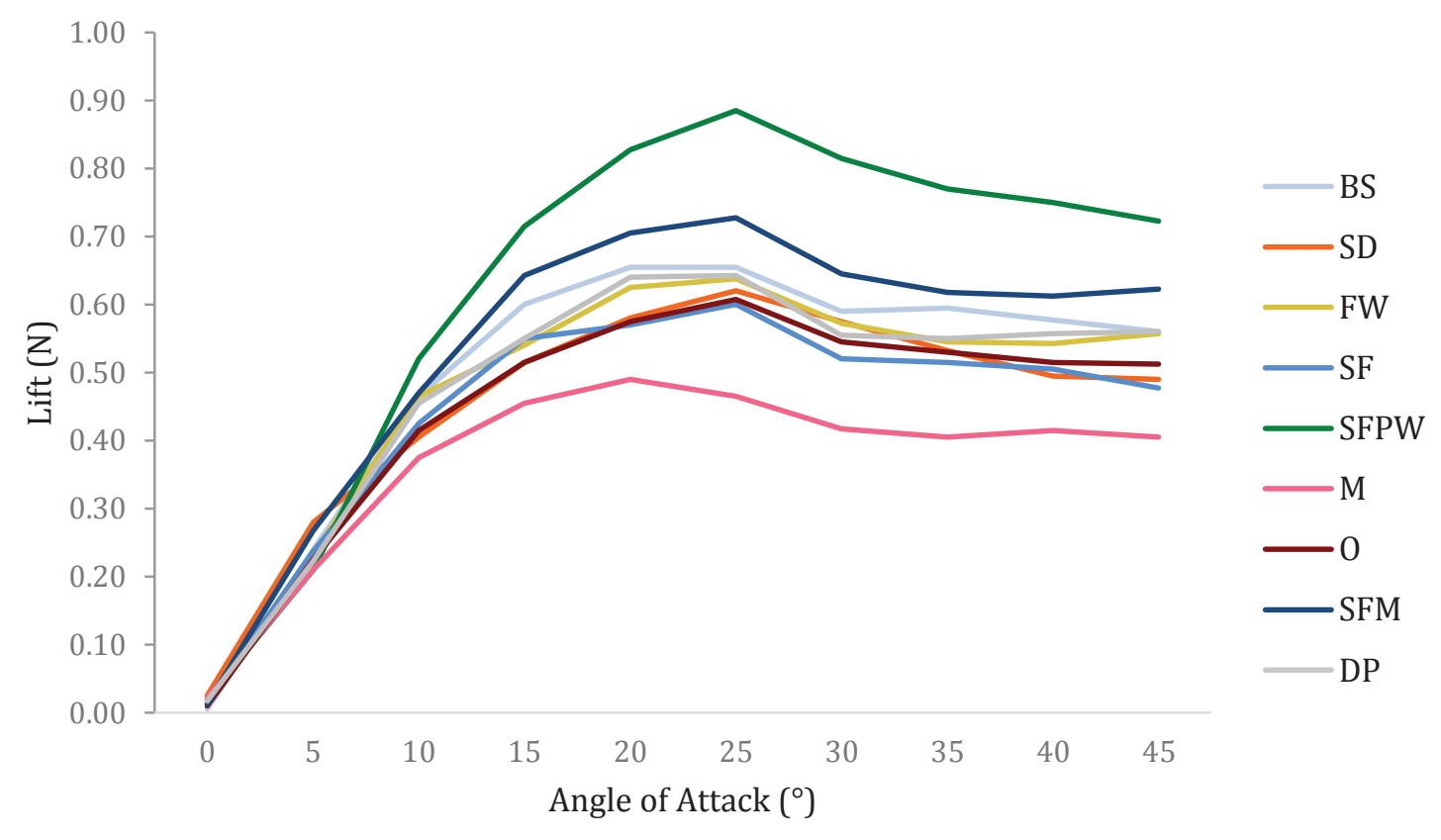

Figure 16: Lift $(\mathrm{N})$ values from experimental data collected for nine fins at ten angles of attack $\left(0-45^{\circ}\right), \mathrm{U}_{\infty}=0.5 \mathrm{mps}$

Table 7 provides the quantitative lift force data from the experimental methods. At $20^{\circ}$ and $25^{\circ}$, peak values $(0.66 \mathrm{~N})$ were equivalent for the BS. The critical angle of attack for the $\mathrm{M}$ was at $20^{\circ}$, with a lift force of $0.49 \mathrm{~N}$. From the critical angle of attack to $45^{\circ}$, the lifting values declined due to hydrofoil stall. This was true for all fins except the FW and SFM. In these cases, the lift forces increased slightly from $40^{\circ}$ to $45^{\circ}$. The DP also showed an irregular trend after the critical angle of attack. The lift force values declined slightly from $25^{\circ}$ to $35^{\circ}$, but then gradually increased from $35^{\circ}$ to $45^{\circ}$. The $\mathrm{M}$ also showed a minor bump at $40^{\circ}(0.42 \mathrm{~N})$, as the lift values increased from $0.41 \mathrm{~N}$ and then returned to $0.41 \mathrm{~N}$ at $45^{\circ}$. 
Table 7: Lift $(\mathrm{N})$ values from experimental data collected for nine fins at ten angles of attack $\left(0-45^{\circ}\right), \mathrm{U}_{\infty}=0.5 \mathrm{mps}$

\begin{tabular}{|l|lllllllll|}
\hline $\boldsymbol{\alpha}\left({ }^{\circ}\right)$ & BS & SD & FW & SF & SFPW & M & O & DP & SFM \\
\hline 0 & 0.01 & 0.03 & 0.02 & 0.02 & 0.02 & 0.02 & 0.01 & 0.02 & 0.01 \\
5 & 0.24 & 0.28 & 0.23 & 0.24 & 0.21 & 0.21 & 0.23 & 0.22 & 0.27 \\
10 & 0.47 & 0.41 & 0.47 & 0.43 & 0.52 & 0.38 & 0.42 & 0.46 & 0.47 \\
15 & 0.60 & 0.52 & 0.54 & 0.55 & 0.72 & 0.46 & 0.52 & 0.55 & 0.64 \\
20 & 0.66 & 0.58 & 0.63 & 0.57 & 0.83 & 0.49 & 0.58 & 0.64 & 0.71 \\
25 & 0.66 & 0.62 & 0.64 & 0.60 & 0.89 & 0.47 & 0.61 & 0.64 & 0.73 \\
30 & 0.59 & 0.58 & 0.57 & 0.52 & 0.82 & 0.42 & 0.55 & 0.56 & 0.65 \\
35 & 0.60 & 0.53 & 0.55 & 0.52 & 0.77 & 0.41 & 0.53 & 0.55 & 0.62 \\
40 & 0.58 & 0.50 & 0.54 & 0.51 & 0.75 & 0.42 & 0.52 & 0.56 & 0.61 \\
45 & 0.56 & 0.49 & 0.56 & 0.48 & 0.72 & 0.41 & 0.51 & 0.56 & 0.62 \\
\hline
\end{tabular}

Note: yellow highlighted quantities represent the highest L/D ratios and blue highlighted quantities represented the lowest $\mathrm{L} / \mathrm{D}$ ratios

There was a correlation between surface area and lift forces. As the surface area increased, the lift forces were also higher in value; refer back to Table 1 for fin dimensions. The SFPW had the greatest surface area of all nine fins and had the highest lift force measurements. This statement is not true for all ten angles of attack. At $0^{\circ}$ and $5^{\circ}$, the SD produced the largest lift force quantities. At $25^{\circ}$, the SFPW had the peak lift force value $(0.89 \mathrm{~N})$ among all the fins for the ten angles of attack. The Marlin yielded the smallest lift forces of all nine fins and it also had the smallest surface area. The SFM had the second highest surface area, with a lifting value reaching $0.73 \mathrm{~N}$ at $25^{\circ}$. Although the DP had a higher surface area compared to the BS, the BS measured greater lift forces at all ten angles of attack.

The surface area of the FW was smaller than the Orca and SD, but produced greater values at several angles. The measured lifting forces of the $\mathrm{FW}$ were found to be greater at $10^{\circ}$, $20^{\circ}$ through $25^{\circ}$, and $35^{\circ}$ through $45^{\circ}$. This irregular trend continued between the Orca and SD. The SD had smaller surface area dimensions compared to the Orca. The SD produced greater lift values, except between $10^{\circ}$ to $15^{\circ}$ and $40^{\circ}$ to $45^{\circ}$. The SF had the smallest surface area between the $\mathrm{O}, \mathrm{SD}$, and FW. This fin produced higher lift forces between $5^{\circ}$ and $15^{\circ}$ compared to the $\mathrm{O}, \mathrm{SD}$, and FW. 


\subsubsection{DRAG FORCE DATA}

Figure 17 shows the drag force curve data at all ten angles of attack for each of the nine fin types. The results provided expected ascending trends that are typical for drag force curves. The drag values increased as the fins' angle of attack moved from zero to $45^{\circ}$. At $45^{\circ}$, the measured drag force values were greatest for all nine fins. This was because at higher angles of attack, a greater surface area was positioned blunt to oncoming flow.

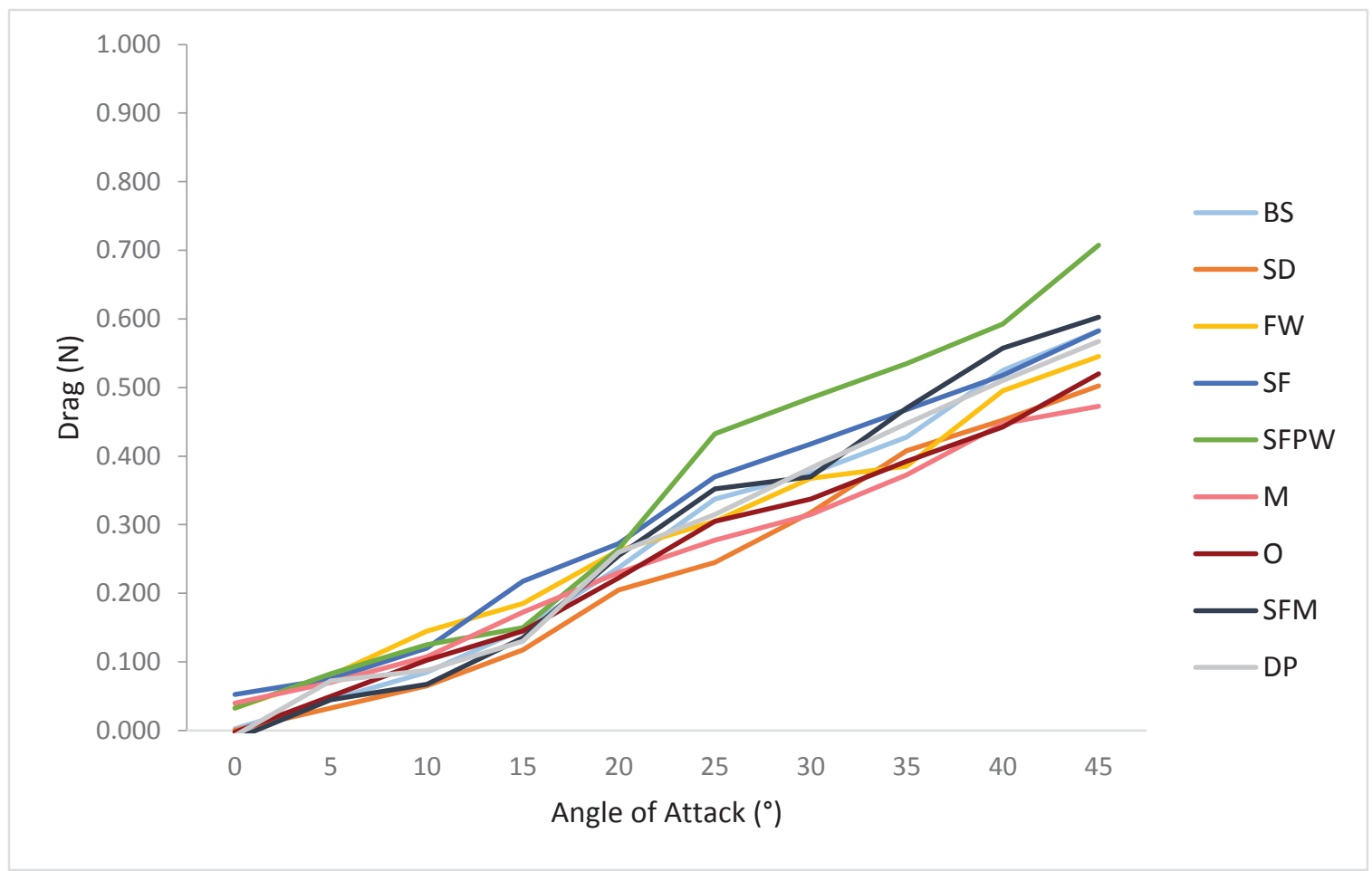

Figure 17: Drag $(\mathrm{N})$ values from experimental data collected for nine fins at ten angles of attack $\left(0-45^{\circ}\right), \mathrm{U}_{\infty}=0.5 \mathrm{mps}$

Table 8 provides raw drag value data from the experimental methods. The relationship between surface area and drag force values was not as clear as the CFD models. Having the largest surface area, the SFPW provided the highest drag values for all angles of attack greater than $15^{\circ}$. At $25^{\circ}$, this fin's drag value was $0.43 \mathrm{~N}$ and $0.71 \mathrm{~N}$ at $45^{\circ}$. The SFPW was surpassed by the $\mathrm{FW}$ at $10^{\circ}(0.15 \mathrm{~N})$, as well as the SF at $0^{\circ}(0.05 \mathrm{~N})$ and $15^{\circ}(0.22 \mathrm{~N})$. 
Table 8: Drag $(\mathrm{N})$ values from experimental data collected for nine fins at ten angles of attack $\left(0-45^{\circ}\right), \mathrm{U}_{\infty}=0.5 \mathrm{mps}$

\begin{tabular}{|l|lllllllll|}
\hline $\boldsymbol{\alpha}\left({ }^{\circ}\right)$ & BS & SD & FW & SF & SFPW & M & O & DP & SFM \\
\hline 0 & 0.00 & 0.00 & 0.03 & 0.05 & 0.03 & 0.04 & 0.00 & -0.01 & 0.01 \\
5 & 0.05 & 0.06 & 0.08 & 0.08 & 0.08 & 0.07 & 0.05 & 0.07 & 0.05 \\
10 & 0.09 & 0.09 & 0.15 & 0.12 & 0.13 & 0.11 & 0.10 & 0.09 & 0.08 \\
15 & 0.15 & 0.13 & 0.19 & 0.22 & 0.15 & 0.17 & 0.15 & 0.13 & 0.14 \\
20 & 0.24 & 0.21 & 0.26 & 0.27 & 0.27 & 0.23 & 0.22 & 0.26 & 0.26 \\
25 & 0.34 & 0.25 & 0.31 & 0.35 & 0.43 & 0.28 & 0.31 & 0.32 & 0.35 \\
30 & 0.38 & 0.32 & 0.37 & 0.39 & 0.49 & 0.32 & 0.34 & 0.38 & 0.37 \\
35 & 0.43 & 0.41 & 0.39 & 0.44 & 0.54 & 0.37 & 0.39 & 0.45 & 0.47 \\
40 & 0.53 & 0.45 & 0.50 & 0.49 & 0.59 & 0.45 & 0.44 & 0.51 & 0.56 \\
45 & 0.58 & 0.50 & 0.55 & 0.52 & 0.71 & 0.47 & 0.52 & 0.57 & 0.60 \\
\hline
\end{tabular}

Note: yellow highlighted quantities represent the highest $\mathrm{L} / \mathrm{D}$ ratios and blue highlighted quantities represented the lowest L/D ratios

At $5^{\circ}$, the BS yielded the smallest drag value $(0.05 \mathrm{~N})$. When the fin was shifted to $10^{\circ}$, the SFM shark produced $0.08 \mathrm{~N}$ drag force, which was the lowest of all nine fins. Although the SD did not have the smallest surface area of all the fins, it had the least amount of drag from $15^{\circ}$ to $25^{\circ}$. With the exception of $40^{\circ}$ angle of attack, the Marlin produced the lowest drag values from $30^{\circ}$ to $45^{\circ}$. At $40^{\circ}$, the Orca produced a drag force of $0.44 \mathrm{~N}$.

\subsubsection{LIFT-TO-DRAG RATIO}

Figure 18 provides calculated L/D ratios from measured experimental data for each of the nine fins. The shapes of the curves were similar to typical L/D ratio trends for symmetric hydrofoil 3D finite wings (fins). 


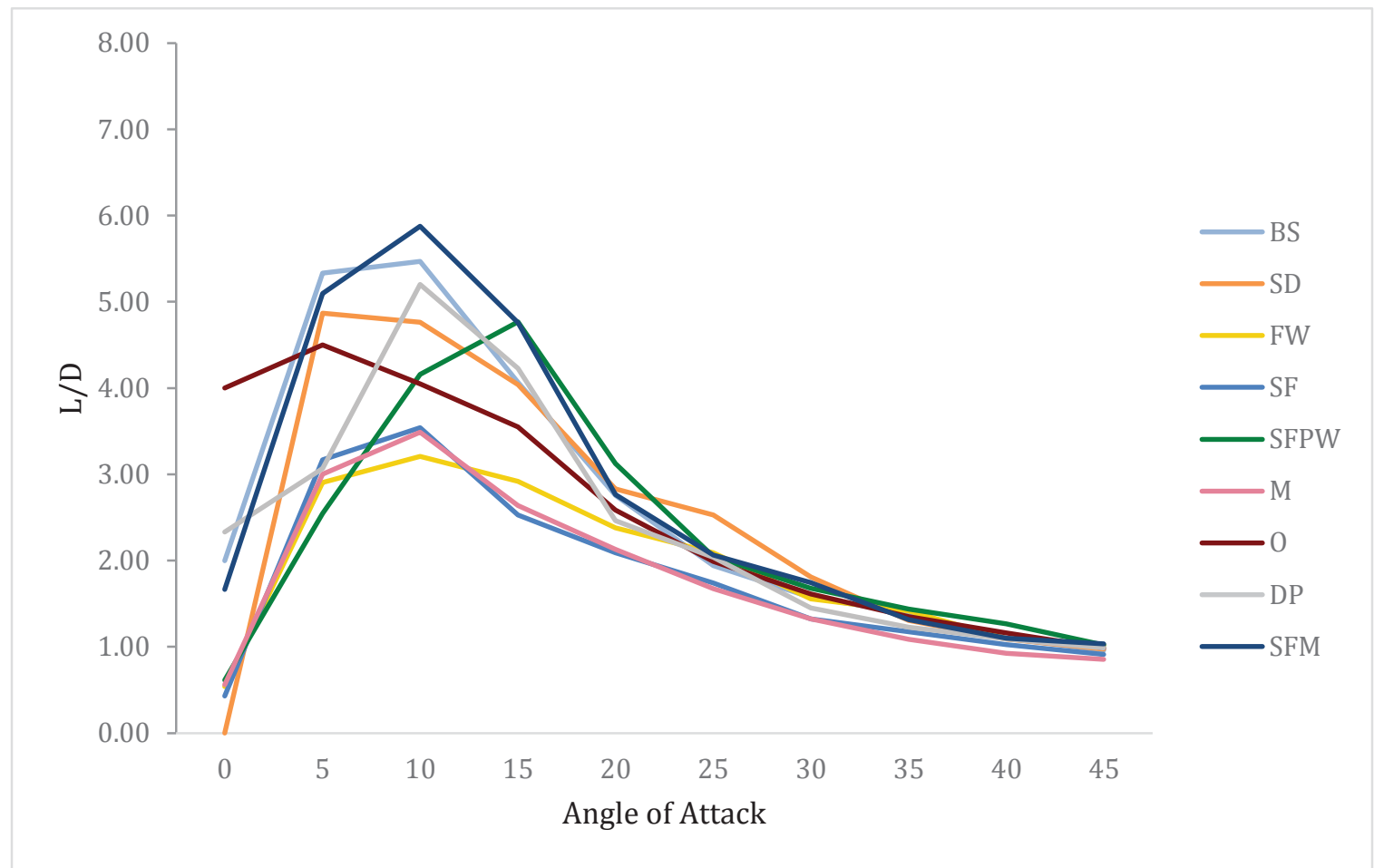

Figure 18: Lift to drag ratio values from experimental data collected for nine fins at ten angles of attack $\left(0-45^{\circ}\right), \mathrm{U}_{\infty}=0.5 \mathrm{mps}$

The numerical data for the L/D ratio curves are presented in Table 9. For each incident angle, the yellow highlighted quantities represented the highest $\mathrm{L} / \mathrm{D}$ ratios and the blue highlighted quantities represented the lowest L/D ratios. Starting from $0^{\circ}$, in sequential order, low efficiency values were generated from the SD, SFPW, FW, and the SF $\left(15^{\circ}\right.$ and $20^{\circ}$ ). At higher angles of attack, the Marlin produced the lowest values. Excluding $35^{\circ}$, the Marlin was the least efficient between $25^{\circ}$ and $45^{\circ}$. The $\mathrm{L} / \mathrm{D}$ ratio was lowest at $0^{\circ}$, reaching a L/D value of 0.00 from the SD. At this angle, the fin with the greatest ratio was the Orca, with a value of 4.00 . This fin was also the most efficient fin at $5^{\circ}$. The FW was the least efficient fin at $10^{\circ}$. At that same angle, the SFM shark produced the peak L/D ratio (5.88) for all nine fins and all ten angles of attack. The SFPW yielded the most peak L/D ratios compared to the other fins. At $15^{\circ}$ through $20^{\circ}$ and $35^{\circ}$ through $40^{\circ}$ the SFPW had the highest $\mathrm{L} / \mathrm{D}$ ratios. 
Table 9: Lift to Drag ratio (L/D) from experimental data collected for nine fins at ten angles of attack $\left(0-45^{\circ}\right), \mathrm{U}_{\infty}=0.5 \mathrm{mps}$

\begin{tabular}{|l|lllllllll|}
\hline $\boldsymbol{\alpha}\left(^{(}\right)$ & BS & SD & FW & SF & SFPW & M & O & DP & SFM \\
\hline 0 & 2.00 & 0.00 & 0.54 & 0.29 & 0.62 & 0.56 & 4.00 & 2.33 & 1.67 \\
5 & 5.33 & 4.87 & 2.91 & 3.17 & 2.55 & 3.00 & 4.50 & 3.07 & 5.10 \\
10 & 5.47 & 4.76 & 3.21 & 3.54 & 4.16 & 3.49 & 4.05 & 5.20 & 5.88 \\
15 & 4.07 & 4.04 & 2.92 & 2.53 & 4.77 & 2.64 & 3.55 & 4.23 & 4.76 \\
20 & 2.76 & 2.83 & 2.38 & 2.09 & 3.12 & 2.13 & 2.58 & 2.46 & 2.76 \\
25 & 1.94 & 2.53 & 2.09 & 1.74 & 2.05 & 1.68 & 1.99 & 2.04 & 2.06 \\
30 & 1.57 & 1.81 & 1.56 & 1.32 & 1.68 & 1.33 & 1.61 & 1.45 & 1.74 \\
35 & 1.39 & 1.31 & 1.42 & 1.18 & 1.44 & 1.09 & 1.35 & 1.23 & 1.31 \\
40 & 1.10 & 1.09 & 1.10 & 1.03 & 1.27 & 0.93 & 1.16 & 1.09 & 1.10 \\
45 & 0.96 & 0.98 & 1.02 & 0.91 & 1.02 & 0.86 & 0.99 & 0.99 & 1.03 \\
\hline
\end{tabular}

Note: yellow highlighted quantities represent the highest $\mathrm{L} / \mathrm{D}$ ratios and blue highlighted quantities represented the lowest L/D ratios

\subsection{DISCUSSION}

Based on the airfoil theory, the experimental results yielded typical lift and drag curve trends (Kundu \& Cohen, 2008). As the angle of attack increased, lift forces grew in value until reaching a critical angle of attack. The drag force intensities developed in size as the incident angles shifted from $0^{\circ}$ to $45^{\circ}$.

As discussed in Section 4.7, the aspect-ratio was not useful in determining the fin with the greatest L/D ratio. This was because the fin's wing span and base chord lengths were the same for all nine fins. Because the shape of the profile changed, the surface area of each fin differed. Due to surface area variations, it was difficult to make any conclusions on the effects of sweep angle.

The relationship between the surface area measurements and force magnitudes were not as clear, compared to the CFD results. Many curves intertwined and did not follow a smooth pattern. This was true for both the lift and drag results. A prime example of entangled lifting force curves was between the Orca, SD, FW, and SF; these fins are presented in order of highest surface area of the four fins to lowest surface area. The weaving of dominant lift 
force values between these four fins could be due to the small differences between the surface area values. It was found that there was only a $10.5 \%$ surface area difference between the Orca and Swordfish. Although the SFPW had the greatest surface area dimensions, the SD produced leading lift forces at $0^{\circ}$ and $5^{\circ}$. This fin had the sixth highest surface area of all the fins. The SFPW had maximum lift forces from $10^{\circ}$ to $45^{\circ}$, with a peaking value of $0.89 \mathrm{~N}$ at $25^{\circ}$. All lift force curves, except for the BS and M, peaked at $25^{\circ}$ critical angle of attack. Unclear trends like these were seen throughout the lift and drag force data gathered in the lab. These uncertainties could be caused by variations between experiments. Each fin case could have encountered a different level of turbulence or small deviation in velocity. Although unlikely, surrounding environmental factors could have effected slight changes in the outcome of each experiment. For instance, small air and water temperature changes could affect the flow. This would cause alterations in the force measurements and ultimately changing efficiency levels.

Efficiency curves formed conventional shapes, but the results did not follow an obvious common trend. Five different fins had peak L/D ratios at different angles of attack, and the same was true for low $\mathrm{L} / \mathrm{D}$ ratios. One fin received the distinction for the highest and lowest $\mathrm{L} / \mathrm{D}$ ratio within the ten angles of attack. At $10^{\circ}$, the SFM had the peak L/D ratio value (5.88) among all the fins for the ten angles of attack. In terms of general surfing conditions, higher lift is desired at lower angles for the ease of manuevering (Gudimetla, Kelson, \& El-Atm, 2009). According to the experimental results, the BS, SFM, and SFPW fulfill these requirements. The SFPW had the most commonly occurring maximum L/D ratio measurements. Four out of the ten angles, the SFPW was the most efficient fin. At lower angles of attack, between $15^{\circ}$ and $20^{\circ}$, the SFPW fin had the greatest L/D ratios. Between $0^{\circ}$ and $5^{\circ}$, the BS was the most efficient fin. The results suggest that the SFPW or the BS would be the most preferrable for the greatest turning and speed on a surfboard for typical surfing conditions (Carswell \& Lavery, 2006).

Big-wave surf requires less manuevering and a greater amounts of stabability at low angles of attack (Gudimetla, Kelson, \& El-Atm, 2009). Lower lift and drag values are more desirable. Out of the ten angles, the Marlin was the least efficient fin at four angles. 
Although the Marlin had the most commonly occurring low L/D ratio measurements of the ten fins, these ratios were at undesirable big wave surfing angles. The SD, SFPW, FW, and $\mathrm{SF}$ generated lowest $\mathrm{L} / \mathrm{D}$ ratios at respective angles $0^{\circ}, 5^{\circ}, 10^{\circ}$, and $15^{\circ}$. These results suggest that the fins might be most useful for directional stability for big-wave surfing conditions. While these L/D ratio values are more suitable for this type of surfing, these four fins did not show consistent low drag values at the small incident angles. The FW, SF, and Marlin are all within about $10 \%$ of the lowest $\mathrm{L} / \mathrm{D}$ ratio for the low incident angles, which can also be seen in Figure 18. These fins had the three lowest surface areas of all nine fins. The FW and the SF did not however, produce the lowest lift forces and drag forces. In fact, these two fins produced the maximum drag forces at $0^{\circ}, 10^{\circ}$, and $15^{\circ}$. The SFPW produced high $\mathrm{L} / \mathrm{D}$ ratio values at all angles of attack, except at $5^{\circ}$.

Though the SD produced the lowest $\mathrm{L} / \mathrm{D}$ ratio at $0^{\circ}(0.00)$, the difference between the lift and drag values was $0.03 \mathrm{~N}$. At this angle, the Orca produced the highest $\mathrm{L} / \mathrm{D}$ ratio of 4.00 , but the difference between the lift and a drag force was only $0.01 \mathrm{~N}$. Because the values at $0^{\circ}$ are so small, large ratios can be produced. There was a $200 \%$ difference between the minimum and maximum $\mathrm{L} / \mathrm{D}$ ratios at $0^{\circ}$, possibly due to turbulence in the flow. Inaccurate readings, caused by human error, could have also provided some margin of error. To improve this, a more accurate data collection and recording device should be used. This would allow more data sets for more precise averages.

Inaccuracies in the data may be influenced by many factors when dealing with experiments in a water channel. The water conditions must be constantly monitored to make sure temperature and water levels stay constant. A source of random error could include high turbulence in the water channel. Due to the size and setup of the water channel and pump, turbulence was an issue. Flow straighteners made of PVC were installed to distribute the water evenly into the flow development section. Unfortunately, the flow straighteners could not support the high volume of water flowing from the inlet section. This caused the inlet section water level to be several inches higher than the flow development and experimental sections. The difference in water heights caused a waterfall effect from the flow straighteners. This created waves and induced turbulence. To fix this issue, a rubber 
mat was fixed into the flume, several inches downstream of the flow straightener section. Water surface undulations were reduced.

The ADV device, to measure the turbulence and velocity, does have a certain margin of error. Because of this, the velocity measurements in the water channel could have been slightly higher or lower than the intended $0.5 \mathrm{mps}$ water speed. The readings could also provide slightly higher or lower turbulence values. Measurement devices are never perfect and all devices have some level of error. The spring gauges used could have provided the largest source of variability. This is because the data was collected through visual analysis instead of a computational data collection system. The spring gauges had a lot of variance, especially at angles near the critical angle of attack. The spring gauge data collector had to decide the range at which the force values stabilized and came to equilibrium. Using this range, an average force value was calculated. Perhaps the length of spring gauge reading time (one minute per reading) was too short or too long. To reduce the systematic biases, a standardized procedure was implemented.

Water channel experiments can be complicated by differentiations between the scaled model and full-scale fins. Comparisons between the experimental and numerical results are not valid here due to the different fin sizes used, respectively. This would include different viscous forces. Higher Reynolds numbers in the scaled models compared to the full size fins. To improve the experiments, the fins should be tested at full size. A larger water channel would be needed so boundary effects would not inhibit the flow around the fins and surfboard. A more advanced data collection system should be used to take instantaneous readings of the data. This way the higher capacity of data collected could be organized and examined to a higher degree of accuracy. This would quantitatively and qualitatively improve the credibility and validity of the study. 


\section{Chapter 6: Optimum Fin}

To properly compare each fin, all variables must be constant. Although each of the fins have equivalent chord length and height dimensions, the profile shapes differ. This caused variation in the surface area and uncomparable force values. To improve this, coefficients of lift $C_{L}$ and coefficients of drag $C_{D}$ were calculated based on wing area $A_{w}$ and upstream velocity $\mathrm{U}_{\infty}$. The non-dimensional numbers allowed the different scaled fins to be assessed by focusing on the ratio of the forces to the dynamic pressure of the oncoming liquid times the area of the fin (Anderson, 2005). This allowed for easy comparisons between the CFD and experimental results in order to find an optimum fin. Referring back to Equation 7 and Equation 8 , the lift and drag force values were used to calculate the coefficients.

This section will provide lift and drag coefficient data from the CFD and laboratory results. Comparisons were made between each fin and the two data gathering methods. Conclusions were drawn in order to select an optimum fin. The results of the CFD highvelocity force values are discussed. The flow patterns were visually and quantitatively analyzed and reported. The results of the remaining eight fins are presented in Appendix C, Figure 102 through Figure 117 and Table 17 through Table 32; the raw data for all nine fins is presented in Table 33 .

\subsection{Result Comparisons}

\subsubsection{CFD LIFT COEFFICIENTS}

As expected, the lift coefficients for the CFD data provided typical lift coefficient curves for a hydrofoil in moving fluid; this is illustrated in Figure 19. As the angle of attack increased from $0^{\circ}$, the lift coefficients increased linearly. The Orca delivered the highest values at these low incident angles. The critical angle of attack was found to be $25^{\circ}$ for all nine fins. This was the angle in which the maximum lift coefficients were found for each fin. At angles higher than the critical angle of attack, stall was produced and decreases in lift coefficients occurred. 
As shown in Table 10, at $25^{\circ}$ the Orca had the peak lift coefficient value (1.22) of the nine fins for all angles of attack. Peaking lift coefficients for each angle of attack are colored in yellow. Conversely, the Marlin had the lowest critical angle of attack value of 1.19. Lowest lift coefficient values were colored in green. Stall was evident as the lift coefficients decreased in value at higher angles of attack. From $30^{\circ}$ to $45^{\circ}$, the SD generated the highest computed values. The SFPW proved to have the lowest coefficient of lift values from $30^{\circ}$ to $35^{\circ}$, while the DP yielded the lowest from $40^{\circ}$ to $45^{\circ}$.

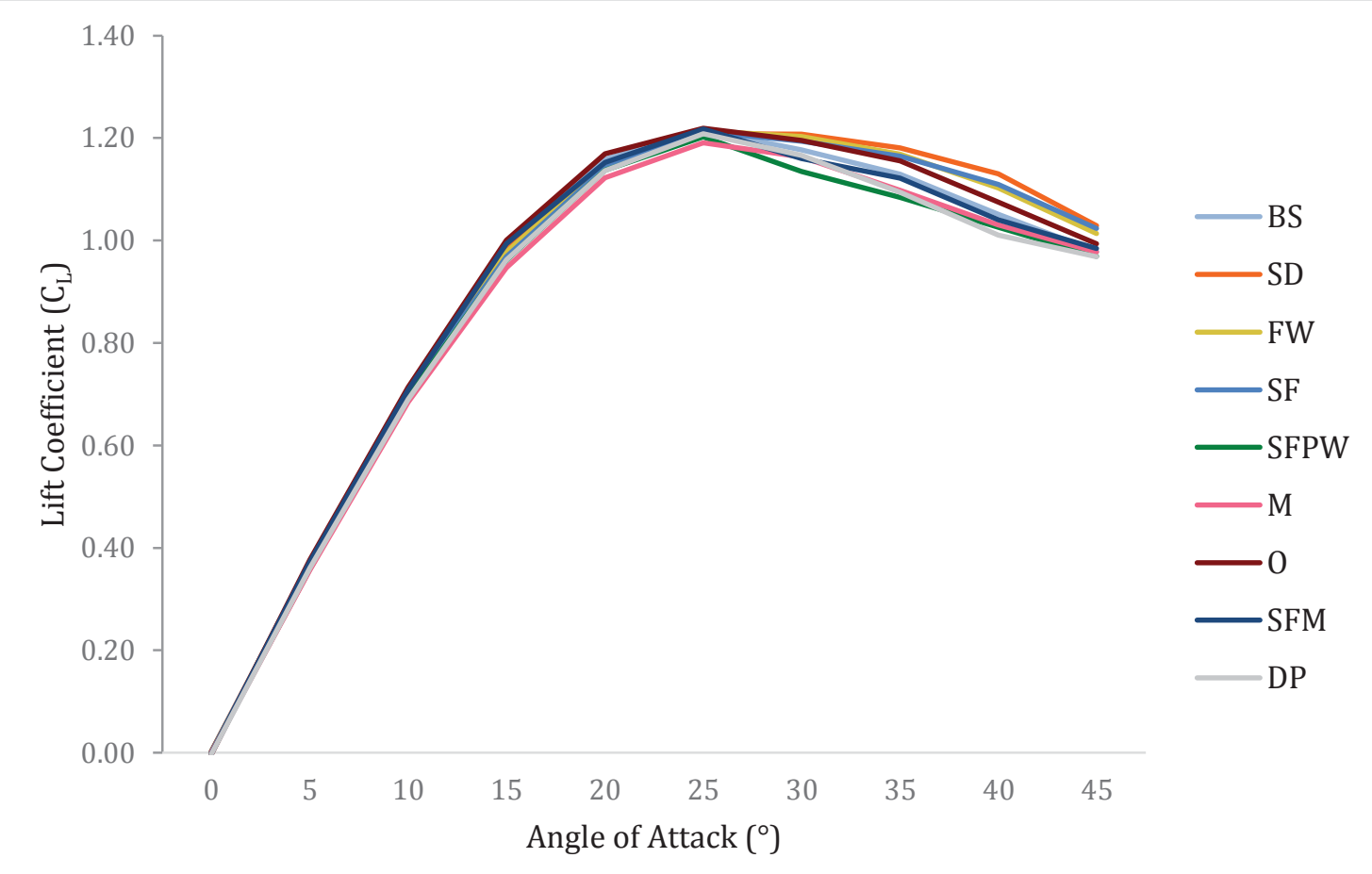

Figure 19: Lift coefficients computed from CFD data collected for nine fins at ten angles of attack $\left(0-45^{\circ}\right), \mathrm{U}_{\infty}=0.5 \mathrm{mps}$ 
Table 10: Lift coefficients computed from CFD data collected for nine fins at ten angles of attack $\left(0-45^{\circ}\right), \mathrm{U}_{\infty}=0.5 \mathrm{mps}$

\begin{tabular}{|l|lllllllll|}
\hline $\boldsymbol{\alpha}\left({ }^{\circ}\right)$ & BS & SD & FW & SF & SFPW & M & O & DP & SFM \\
\hline 0 & 0.00 & 0.00 & 0.00 & 0.00 & 0.00 & 0.00 & 0.00 & 0.00 & 0.00 \\
5 & 0.37 & 0.37 & 0.37 & 0.37 & 0.36 & 0.36 & 0.38 & 0.36 & 0.37 \\
10 & 0.71 & 0.71 & 0.70 & 0.70 & 0.70 & 0.68 & 0.71 & 0.69 & 0.71 \\
15 & 0.99 & 0.98 & 0.98 & 0.97 & 0.96 & 0.95 & 1.00 & 0.96 & 0.99 \\
20 & 1.16 & 1.15 & 1.15 & 1.14 & 1.14 & 1.12 & 1.17 & 1.14 & 1.15 \\
25 & 1.22 & 1.21 & 1.21 & 1.21 & 1.20 & 1.19 & 1.22 & 1.21 & 1.22 \\
30 & 1.18 & 1.21 & 1.20 & 1.19 & 1.13 & 1.16 & 1.20 & 1.17 & 1.16 \\
35 & 1.13 & 1.18 & 1.17 & 1.16 & 1.08 & 1.10 & 1.16 & 1.09 & 1.12 \\
40 & 1.05 & 1.13 & 1.10 & 1.11 & 1.03 & 1.03 & 1.08 & 1.01 & 1.04 \\
45 & 0.98 & 1.03 & 1.01 & 1.02 & 0.97 & 0.98 & 0.99 & 0.97 & 0.98 \\
\hline
\end{tabular}

Note: yellow highlighted quantities represent the highest L/D ratios and blue highlighted quantities represented the lowest L/D ratios

\subsubsection{LAB LIFT COEFFICIENTS}

The laboratory lift force results were calculated and presented as lift coefficients, displayed in Figure 20 and Table 11. The curve formations followed typical lift coefficient curves, but the CFD and lab results were not congruent.

Unlike the CFD results, the laboratory critical angle of attack varied between all nine fins. Most of the fins produced critical lift coefficient values at $25^{\circ}$. Stall occurred after $20^{\circ}$ for the Marlin. This critical value (1.14) was also the lowest for all nine fins. The BS had identical critical lift coefficient values (1.23) at $20^{\circ}$ and $25^{\circ}$. The FW had the peak lift coefficient value (1.33) of all nine fins for all angles of attack at $25^{\circ}$. Comparing this value to the CFD results, the peak lift coefficient was only 1.22 from the Orca. Although this was only an $8.6 \%$ difference, the fin shapes were dissimilar. While the Orca provided the highest CFD lift coefficient values from $0^{\circ}$ to $25^{\circ}$, this fin's lift coefficient values never dominated the other fins in the laboratory results. For the CFD results, the Marlin consistently yielded the lowermost lift coefficient quantities from $0^{\circ}$ to $25^{\circ}$, and provided the lowest lift coefficients from $20^{\circ}$ to $45^{\circ}$ for the lab experiments. Sharing the lowest quantities at $20^{\circ}$ and $25^{\circ}$, the values for each of these angles were extremely close. At $20^{\circ}$, there was only a $1.3 \%$ difference between the two data collection methods (lab and CFD) and a $9.8 \%$ difference at $25^{\circ}$. 


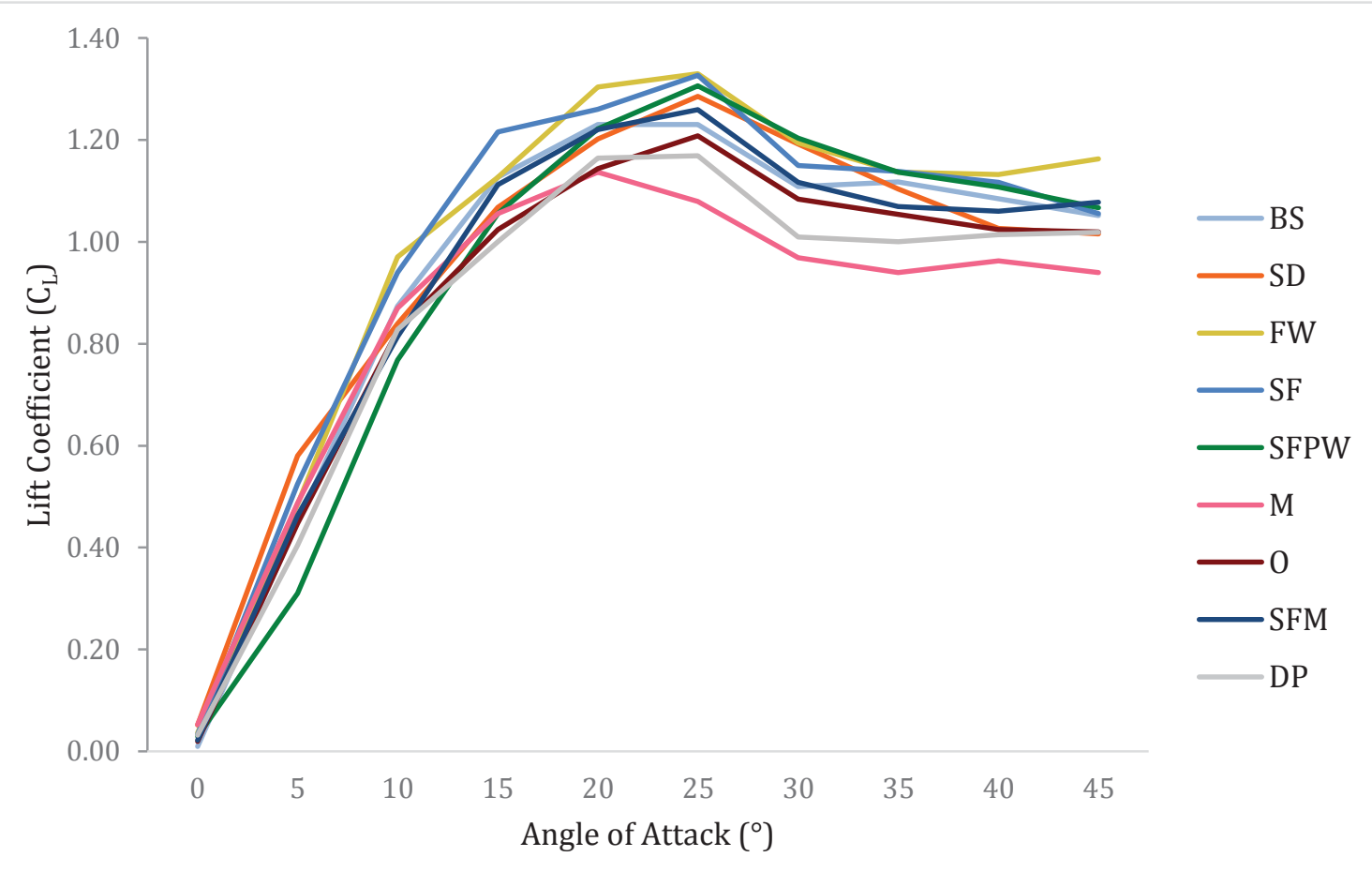

Figure 20: Lift coefficients computed from lab data collected for nine fins at ten angles of attack $\left(0-45^{\circ}\right), \mathrm{U}_{\infty}=0.5 \mathrm{mps}$

Based on the lab data, the rankings of the fins also conflicted with the CFD results. As the lift coefficient values increased towards the critical angle, several fins showed local peaks at each angle. From $0^{\circ}$ to $5^{\circ}$, the SD had the greatest lift coefficient values, while the FW had greater values at $10^{\circ}, 20^{\circ}$, and $25^{\circ}$. The SF exhibited a local peak lift coefficient value of 1.22 at $15^{\circ}$. At higher angles of attack, the SFPW, the SF, and the FW overlapped one another for the highest lift coefficient values. At $30^{\circ}$, the SFPW created the highest value (1.20) from the lab results, but had the lowest value (1.13) at that angle from the CFD results. While the SF produced a local peak factor (1.14) at $35^{\circ}$ for the lab results, the coefficient of lift value was higher for the CFD results at that angle (1.16); there was only a $2 \%$ difference between the two methods. Between $40^{\circ}$ and $45^{\circ}$, the $\mathrm{FW}$ had the highest lift coefficient quantities, 1.13 and 1.16 respectively. 
Table 11: Lift coefficients computed from lab data collected for nine fins at ten angles of attack $\left(0-45^{\circ}\right), \mathrm{U}_{\infty}=0.5 \mathrm{mps}$

\begin{tabular}{|l|lllllllll|}
\hline $\boldsymbol{\alpha}\left(^{\circ}\right)$ & BS & SD & FW & SF & SFPW & M & O & DP & SFM \\
\hline 0 & 0.01 & 0.05 & 0.04 & 0.03 & 0.03 & 0.05 & 0.02 & 0.03 & 0.02 \\
5 & 0.45 & 0.58 & 0.49 & 0.53 & 0.31 & 0.49 & 0.45 & 0.40 & 0.46 \\
10 & 0.87 & 0.84 & 0.97 & 0.94 & 0.77 & 0.87 & 0.83 & 0.83 & 0.81 \\
15 & 1.13 & 1.07 & 1.13 & 1.22 & 1.06 & 1.06 & 1.02 & 1.00 & 1.11 \\
20 & 1.23 & 1.20 & 1.30 & 1.26 & 1.22 & 1.14 & 1.14 & 1.16 & 1.22 \\
25 & 1.23 & 1.29 & 1.33 & 1.33 & 1.31 & 1.08 & 1.21 & 1.17 & 1.26 \\
30 & 1.11 & 1.19 & 1.19 & 1.15 & 1.20 & 0.97 & 1.08 & 1.01 & 1.12 \\
35 & 1.12 & 1.10 & 1.14 & 1.14 & 1.14 & 0.94 & 1.05 & 1.00 & 1.07 \\
40 & 1.08 & 1.03 & 1.13 & 1.12 & 1.11 & 0.96 & 1.02 & 1.01 & 1.06 \\
45 & 1.05 & 1.02 & 1.16 & 1.06 & 1.07 & 0.94 & 1.02 & 1.02 & 1.08 \\
\hline
\end{tabular}

Note: yellow highlighted quantities represent the highest $\mathrm{L} / \mathrm{D}$ ratios and blue highlighted quantities represented the lowest $\mathrm{L} / \mathrm{D}$ ratios

The lab lift coefficient results were generally higher compared to the CFD results. The percent variation was smaller at higher angles of attack. For six fins, there was less than a $10 \%$ difference from $20^{\circ}$ to $45^{\circ}$. The DP, FW, and Marlin showed high variation at several incident angles. The DP had differences of less than 10\% for almost all ten angles of attack, excluding $0^{\circ}$ to $10^{\circ}$ and $30^{\circ}$. At $40^{\circ}$, the DP produced the lowest variation of $0.3 \%$ between the CFD (1.01) and lab (1.01) lift coefficient results.

\subsubsection{CFD DRAG COEFFICIENTS}

The calculated drag coefficients from the CFD results followed a typical ascending trend, which was expected for drag coefficient curves of a streamlined fin. This is presented in Figure 21 and quantitatively valued in Table 12. 


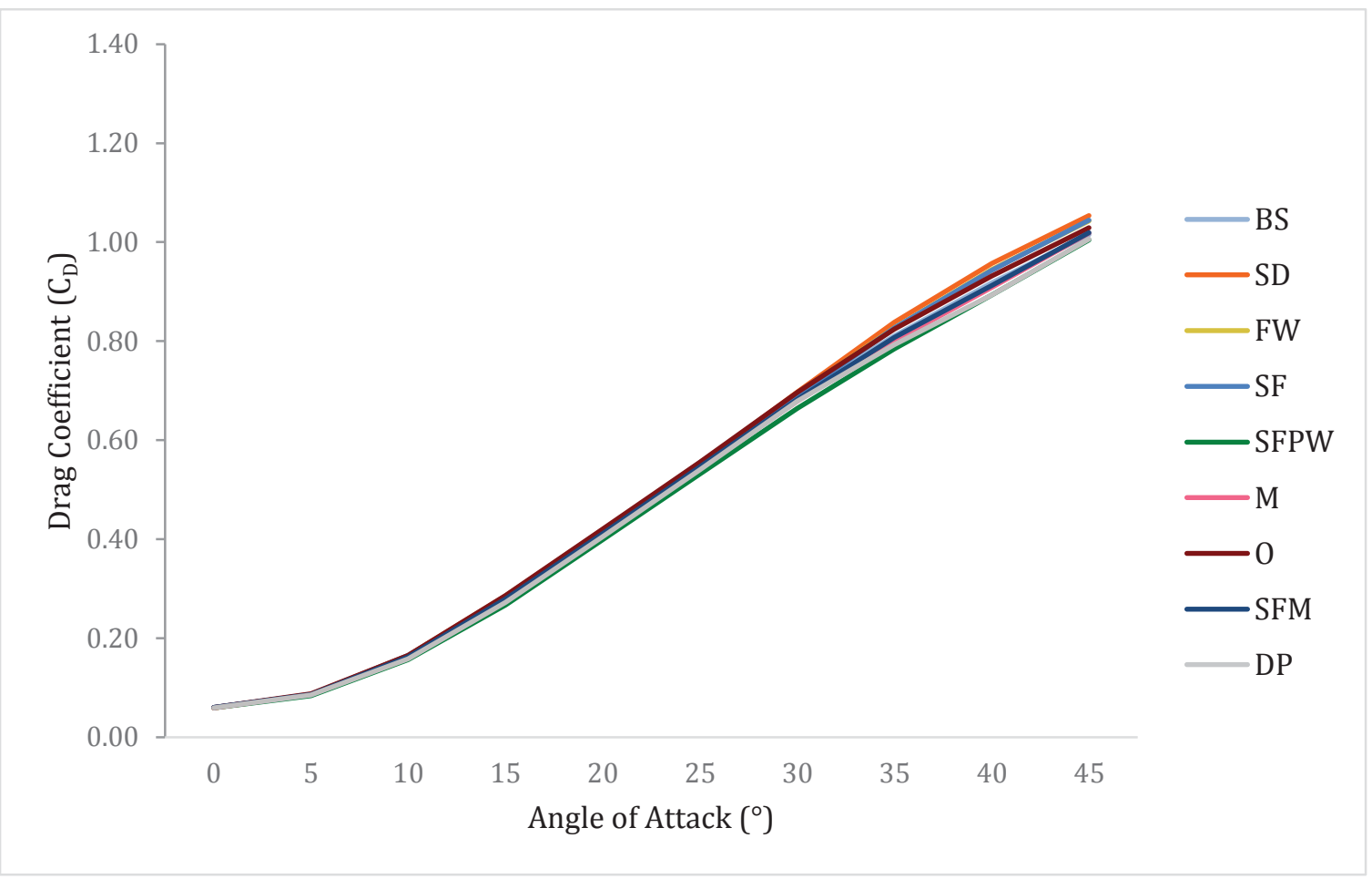

Figure 21: Drag coefficients computed from CFD data collected for nine fins at ten angles of attack $\left(0-45^{\circ}\right), \mathrm{U}_{\infty}=0.5 \mathrm{mps}$

As the angle of attack increased towards $45^{\circ}$, the drag coefficients grew in magnitude. At all angles of attack, the SFPW exhibits the lowest drag coefficient values. This was the only consistent trend within this section of the data. The DP provided the second lowest drag coefficient values at all angles of attack, except at $30^{\circ}$. The third lowest of the coefficients was between the SFM, occurring from $5^{\circ}$ to $10^{\circ}$, and the Marlin, occurring from $15^{\circ}$ to $45^{\circ}$. For the rest of the rankings, the fins intertwined and did not show any consistent tendencies. The Orca yielded the highest of the drag coefficients from $0^{\circ}$ to $25^{\circ}$, while the SD computed the highest values from $30^{\circ}$ to $45^{\circ}$. The highest drag value (1.06) was produced at $45^{\circ}$ from the SD. 
Table 12: Drag coefficients computed from CFD data collected for nine fins at ten angles of attack $\left(0-45^{\circ}\right), \mathrm{U}_{\infty}=0.5 \mathrm{mps}$

\begin{tabular}{|l|lllllllll|}
\hline $\boldsymbol{\alpha}\left({ }^{\circ}\right)$ & BS & SD & FW & SF & SFPW & M & O & DP & SFM \\
\hline 0 & 0.06 & 0.06 & 0.06 & 0.06 & 0.06 & 0.06 & 0.06 & 0.06 & 0.06 \\
5 & 0.09 & 0.09 & 0.09 & 0.09 & 0.08 & 0.09 & 0.09 & 0.09 & 0.09 \\
10 & 0.16 & 0.16 & 0.16 & 0.17 & 0.16 & 0.16 & 0.17 & 0.16 & 0.16 \\
15 & 0.28 & 0.29 & 0.28 & 0.28 & 0.27 & 0.28 & 0.29 & 0.27 & 0.28 \\
20 & 0.42 & 0.42 & 0.42 & 0.42 & 0.40 & 0.41 & 0.42 & 0.41 & 0.41 \\
25 & 0.55 & 0.55 & 0.55 & 0.55 & 0.53 & 0.54 & 0.56 & 0.54 & 0.55 \\
30 & 0.69 & 0.70 & 0.69 & 0.69 & 0.66 & 0.68 & 0.70 & 0.68 & 0.68 \\
35 & 0.81 & 0.84 & 0.83 & 0.83 & 0.78 & 0.80 & 0.82 & 0.79 & 0.81 \\
40 & 0.92 & 0.96 & 0.94 & 0.94 & 0.89 & 0.91 & 0.93 & 0.89 & 0.91 \\
45 & 1.02 & 1.05 & 1.04 & 1.04 & 1.00 & 1.02 & 1.03 & 1.01 & 1.02 \\
\hline
\end{tabular}

Note: yellow highlighted quantities represent the highest L/D ratios and blue highlighted quantities represented the lowest $\mathrm{L} / \mathrm{D}$ ratios

\subsubsection{LAB DRAG COEFFICIENTS}

Focusing on the laboratory drag coefficients in Figure 22 and Table 13, the data showed similar ascending trends towards $45^{\circ}$ angle of attack. The data did not however, follow the same patterns as found in the CFD drag coefficient results.

Six different fins provided the lowest coefficient values at each angle of attack. The BS had the lowest value $(0.08)$ at $5^{\circ}$ and the SFM calculated the smallest coefficient (0.14) at $10^{\circ}$. The SFPW yielded the lowest local drag coefficients at $15^{\circ}$ and $20^{\circ}, 0.22$ and 0.39 respectively. At $25^{\circ}$ the $\mathrm{SD}$ produced a value of 0.55 and at $30^{\circ}$ the SFM had the smallest value of 0.64 . The Orca gave values of 0.78 and 0.88 at $35^{\circ}$ and $40^{\circ}$. At $45^{\circ}$, the DP provided the lowest drag coefficient of 1.03 for all nine fins.

The data did not produce a fin that followed any consistent trends. The curves intertwined from $0^{\circ}$ to $45^{\circ}$, except for the SF. The SF had the highest drag coefficient values from $15^{\circ}$ to $45^{\circ}$, peaking at $45^{\circ}$ with a value of 1.16 . The FW had the highest drag coefficient values at $5^{\circ}$ and $10^{\circ}$, while the Marlin had the highest at $0^{\circ}$. Averaging each fin for all angles of attack, the SF resulted in the highest average while the SD had the lowest average. 


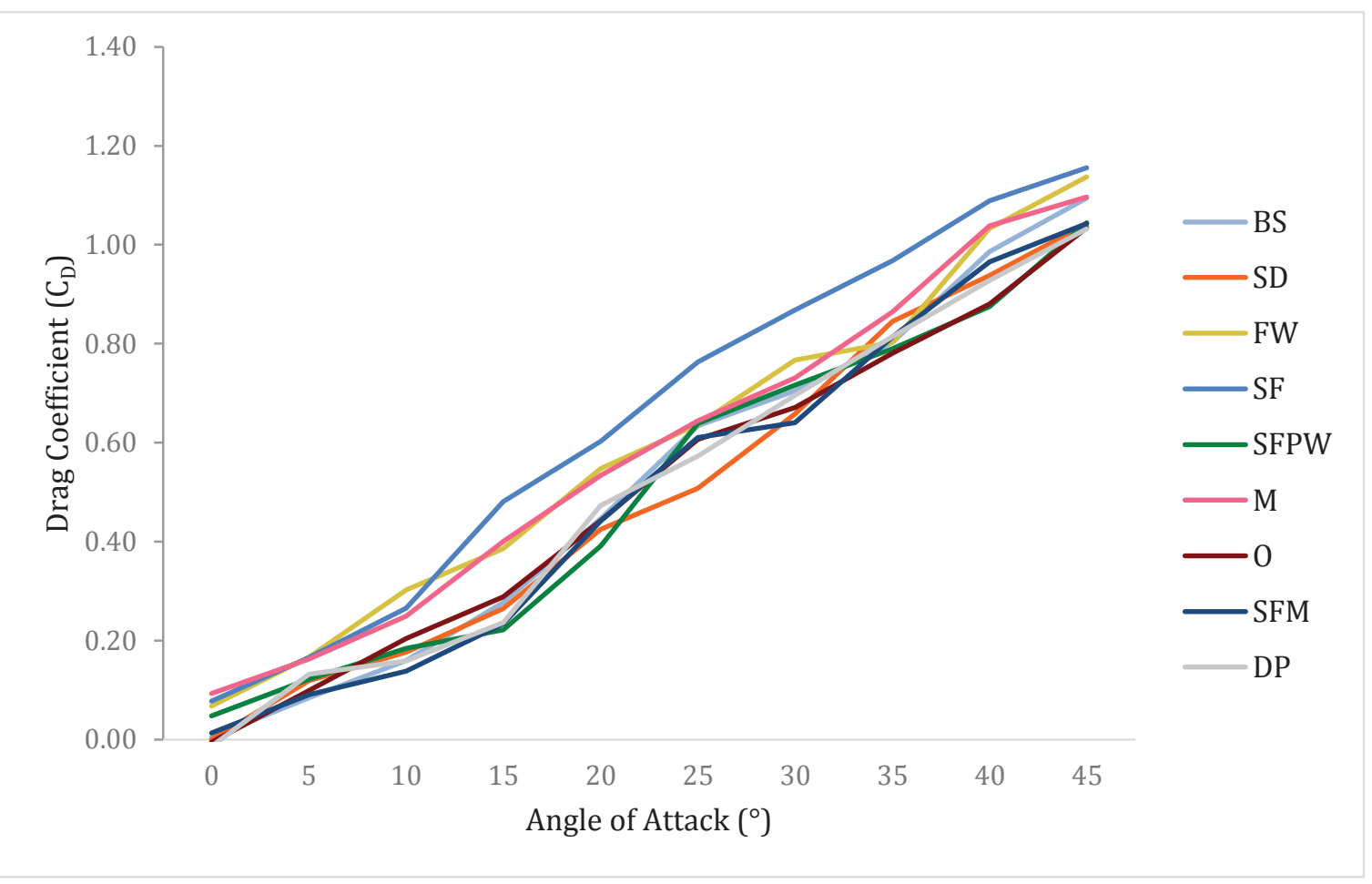

Figure 22: Drag coefficients computed from lab data collected for nine fins at ten angles of attack $\left(0-45^{\circ}\right), \mathrm{U}_{\infty}=0.5 \mathrm{mps}$

Table 13: Drag coefficients computed from lab data collected for nine fins at ten angles of attack $\left(0-45^{\circ}\right), U_{\infty}=0.5 \mathrm{mps}$

\begin{tabular}{|l|lllllllll|}
\hline $\boldsymbol{\alpha}\left({ }^{\circ}\right)$ & BS & SD & FW & SF & SFPW & M & 0 & DP & SFM \\
\hline 0 & 0.00 & 0.00 & 0.07 & 0.08 & 0.05 & 0.09 & 0.00 & -0.01 & 0.01 \\
5 & 0.08 & 0.12 & 0.17 & 0.17 & 0.12 & 0.16 & 0.10 & 0.13 & 0.09 \\
10 & 0.16 & 0.18 & 0.30 & 0.27 & 0.18 & 0.25 & 0.20 & 0.16 & 0.14 \\
15 & 0.28 & 0.26 & 0.39 & 0.48 & 0.22 & 0.40 & 0.29 & 0.24 & 0.23 \\
20 & 0.45 & 0.42 & 0.55 & 0.60 & 0.39 & 0.53 & 0.44 & 0.47 & 0.44 \\
25 & 0.63 & 0.51 & 0.64 & 0.76 & 0.64 & 0.64 & 0.61 & 0.57 & 0.61 \\
30 & 0.70 & 0.66 & 0.77 & 0.87 & 0.72 & 0.73 & 0.67 & 0.70 & 0.64 \\
35 & 0.80 & 0.84 & 0.80 & 0.97 & 0.79 & 0.86 & 0.78 & 0.81 & 0.81 \\
40 & 0.99 & 0.94 & 1.03 & 1.09 & 0.87 & 1.04 & 0.88 & 0.93 & 0.97 \\
45 & 1.09 & 1.04 & 1.14 & 1.16 & 1.04 & 1.10 & 1.03 & 1.03 & 1.04 \\
\hline
\end{tabular}

Note: yellow highlighted quantities represent the highest $\mathrm{L} / \mathrm{D}$ ratios and blue highlighted quantities represented the lowest $\mathrm{L} / \mathrm{D}$ ratios

In general, the laboratory drag coefficients were slightly higher than the CFD results. Differences tended to be greater at lower angles of attack. Excluding the SF and the Marlin, 
from $30^{\circ}$ to $45^{\circ}$, percent differences were less than $10 \%$. The SF had the highest variation between the two methods for almost all angles of attack. The greatest difference was found at $0^{\circ}$ and $5^{\circ}$ for all nine fins. The BS shared several similarities in drag coefficient values between the two methods. Between $5^{\circ}$ and $45^{\circ}$, there was on average a $3.5 \%$ difference in value between the two methods. There was a less than $10 \%$ variation between the CFD and lab coefficients for the SD from $10^{\circ}$ to $45^{\circ}$. This trend was also true for the Orca between $15^{\circ}$ and $45^{\circ}$. The drag coefficient comparisons varied more than the lift coefficient results between the two methods of data collection.

\subsubsection{LIFT-TO-DRAG RATIOS}

The lift-to-drag ratios were the same for the force values and coefficient values. Referring back to Section 4.1 and Section 5.1, the L/D ratio tables and figures were used to make final comparisons and conclusions.

The purpose of these experiments was to find a fin with the greatest lift-to-drag ratio. This would mean a fin that had high lift values and low drag values in order to allow a surfer to maneuver a surfboard and single fin setup with ease for small to average surfing conditions. While there is not one fin that produced a high L/D ratio at all angles of attack, there was one fin that generated higher L/D ratios in both the CFD and laboratory results than any of the other nine designed fins. This fin was the SFPW and had the most commonly occurring highest $\mathrm{L} / \mathrm{D}$ ratio among the ten angles of attack compared to all nine fins.

In Figure 26 and Table 6, the results of the CFD L/D ratios are shown. The SFPW produced the highest $\mathrm{L} / \mathrm{D}$ ratios from $10^{\circ}$ to $25^{\circ}$. The fin was most efficient at $10^{\circ}$, with a value of 4.45. Comparing this to the laboratory results in Figure 29 and Table 9, the SFPW had the highest $\mathrm{L} / \mathrm{D}$ ratio values at $15^{\circ}$ to $20^{\circ}$ and $30^{\circ}$ to $40^{\circ}$. The CFD and lab results shared common peaks at $15^{\circ}$ and $20^{\circ}$. At $15^{\circ}$, there was a $28.1 \%$ difference between the CFD ratio (3.59) and the lab ratio (4.77). On the other hand, there was only a 9.1\% difference between the CFD results (2.85) and the lab results (3.12) for $20^{\circ}$ angle of attack. Between these two methods, several of the values were very close in value. At $35^{\circ}$, the CFD produced a 1.38 ratio and the lab L/D ratio was 1.27 . Only a $1.7 \%$ difference lied between the CFD (1.71) and lab (1.68) $\mathrm{L} / \mathrm{D}$ ratios at $30^{\circ}$. 


\subsection{OPTIMUM Fin RESUlts}

\subsubsection{SFPW LIFT COEFFICIENTS}

The optimum fin chosen was the SFPW. This was because it had the most commonly occurring high $\mathrm{L} / \mathrm{D}$ ratio in both the $\mathrm{CFD}$ and lab results. The variances between the CFD and lab results for the SFPW are shown in the following figures; Figure 23 and Figure 24. By comparing both methods through the lift and drag coefficient data, more can be understood about the commonalities and differentiations between the $\mathrm{L} / \mathrm{D}$ ratios.

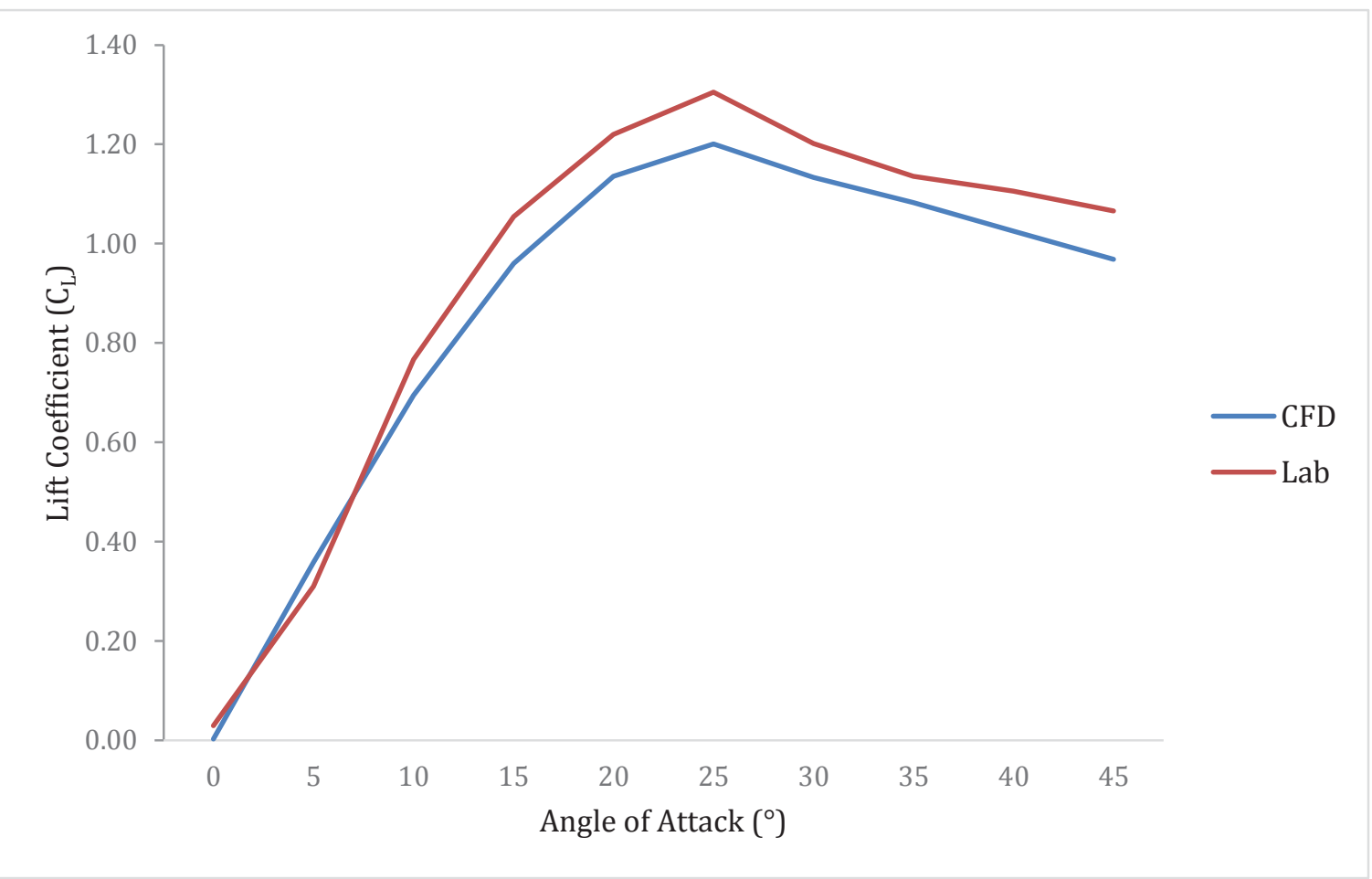

Figure 23: Coefficient of lift from experimental and CFD data collected for the SFPW at ten angles of attack $\left(0-45^{\circ}\right), \mathrm{U}_{\infty}=0.5 \mathrm{mps}$

Lifting values were greater in the CFD results because the SFPW fin was scaled 2.3 times larger than the laboratory fin. Using the lift coefficients, size was no longer a factor and the two methods could be assessed. Due to dissimilar Re numbers between the methods, the results could not be compared appropriately. For the purpose of this project, associations were made between the results of the two methods with understanding that $\mathrm{Re}$ 
numbers were not equivalent. For almost all angles of attack, the lab results produced slightly higher lift coefficient values.

As stated earlier, both methods follow a common lift coefficient curve trend. The values increase from $0^{\circ}$ towards a peak lift value at the critical angle of attack. Both sets of data reached the summit of the curve at $25^{\circ}$. Here, there was an $8.3 \%$ difference between the CFD results (1.20) and the lab results (1.31). The coefficients then decrease after stall has occurred, as higher incident angles are encountered. The laboratory lift coefficients were higher in value at all angles of attack, excluding $5^{\circ}$ angle of attack. At this angle, the lab results dipped below the CFD results, producing a $14.5 \%$ difference between the two methods. With only a $4.8 \%$ disagreement, the data was closest in value at $35^{\circ}$.

\subsubsection{SFPW DRAG COEFFICIENTS}

The drag coefficient data also follows a typical trend, but the patterns do not coincide between the CFD and lab results. The CFD values incline steadily as the incident angles grow. Focusing on the laboratory results, the slope of the line varies in gradient. It is a gradual $1.2 \%$ incline from $0^{\circ}$ to $15^{\circ}$, but transitions to a more steep $4.2 \%$ gradient from $15^{\circ}$ to $25^{\circ}$. This causes the curve to cross back and forth over the CFD curve. From $25^{\circ}$ to $40^{\circ}$, the curve stabilizes to a $1.6 \%$ incline, causing the line to settle below the CFD drag coefficient curve. As the laboratory drag coefficient curve moves from $40^{\circ}$ to $45^{\circ}$, the values once again surpass the CFD coefficients.

Between the two methods, the drag coefficients are not as closely related at smaller angles of incidents, but show similar values at higher angles of attack. At $5^{\circ}$, the greatest variation between the CFD (0.08) and lab (0.12) coefficients is found and was calculated at $37.0 \%$. The difference in value is less than 0.04 though. At $15^{\circ}$ and $25^{\circ}$, percent differences are about $18 \%$, but from $30^{\circ}$ to $45^{\circ}$ the difference is less than $8 \%$. There is only a $0.70 \%$ variation between the two methods of data collection at $35^{\circ}$. The CFD results produced a drag coefficient of 0.78 , while the lab coefficient was 0.79 . The greatest difference in value was found to be 0.05 at $30^{\circ}$. 


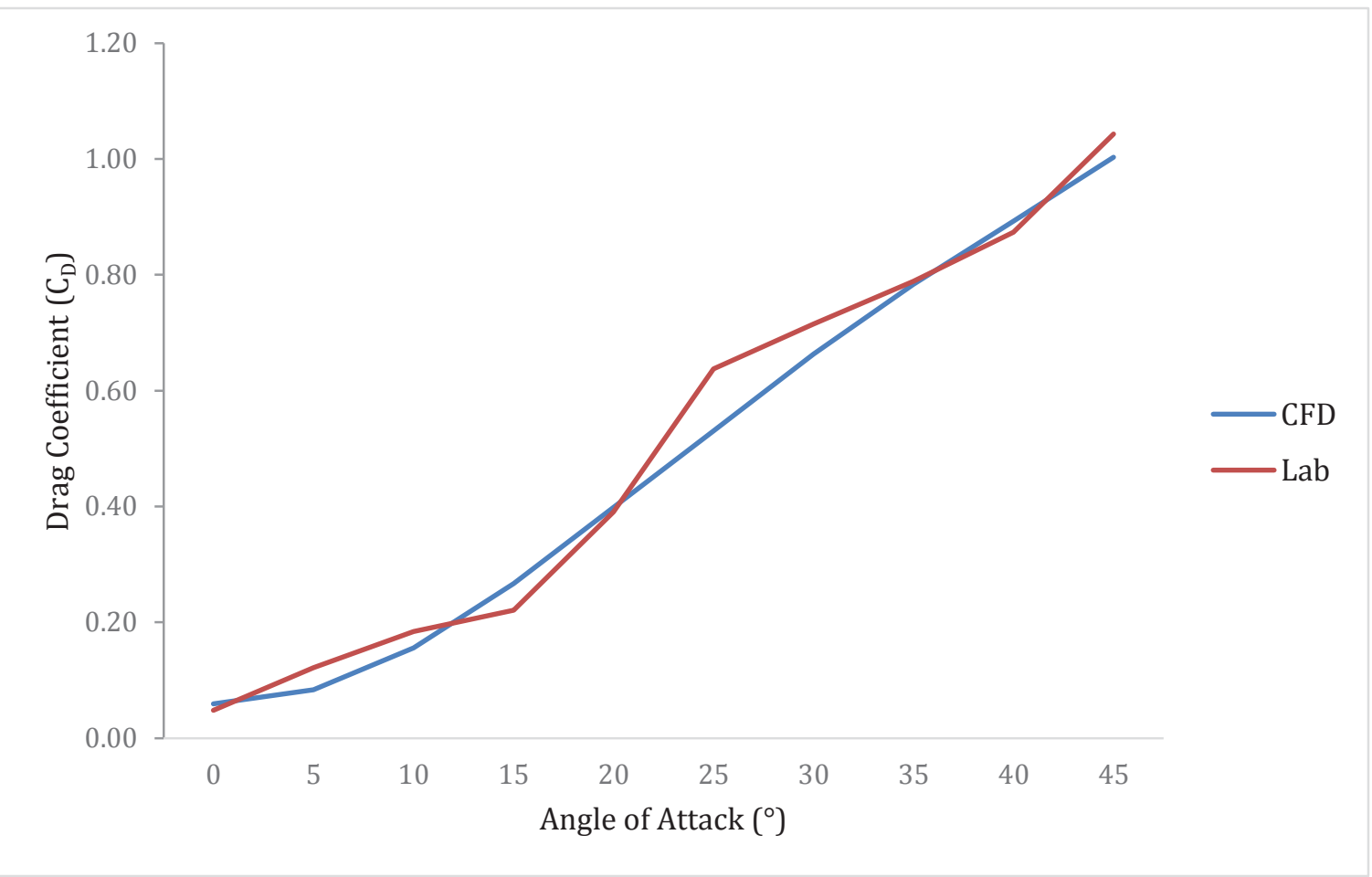

Figure 24: Coefficient of drag from experimental and CFD data collected for the SFPW at ten angles of attack $\left(0-45^{\circ}\right), \mathrm{U}_{\infty}=0.5 \mathrm{mps}$

\subsubsection{HIGH VELOCITY ANALYSIS}

The three high velocity values chosen were $3 \mathrm{mps}$, 5mps, and $7 \mathrm{mps}$. These were based on typical surfing velocities measured for $\sim 0.5$ to $2.5 \mathrm{~m}$ wave heights according to Hendricks (1969) and Edge (2001). Figure 25 presents the data gathered from the CFD simulation results.

At higher velocities, greater lift forces were generated. At $25^{\circ}$ angle of attack, the SFPW had the highest lift force of $377.6 \mathrm{~N}$ under a velocity of $7 \mathrm{mps}$. The SFPW peaked at this same angle for the upstream velocities of $5 \mathrm{mps}(192.7 \mathrm{~N})$ and $3 \mathrm{mps}(69.4 \mathrm{~N})$. The lift force curves provided similar shapes compared to one another. These curves also followed the same trend and shape as the low velocity lift force curve for the SFPW CFD results in Figure 24. 


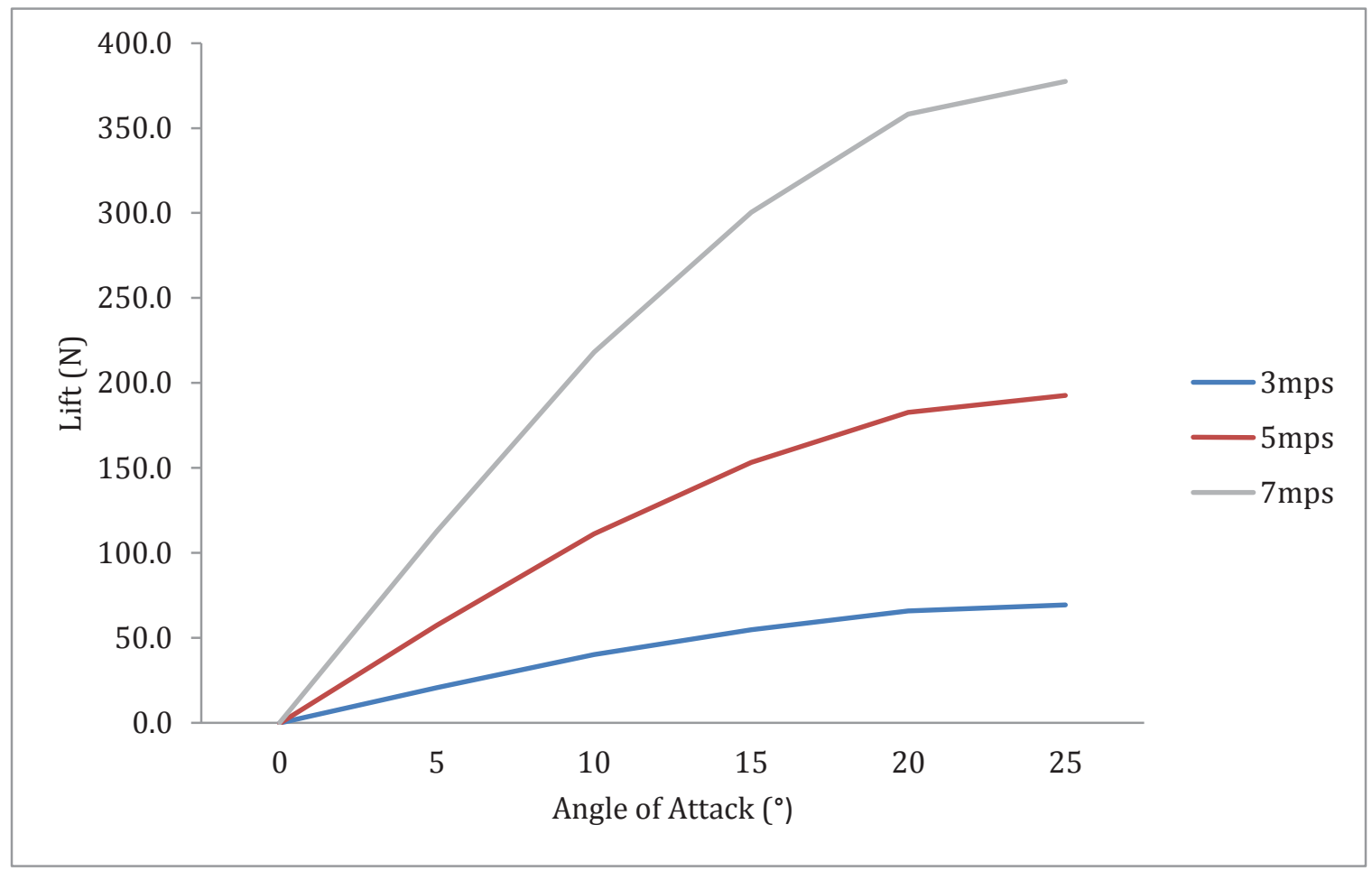

Figure 25: Lift $(\mathrm{N})$ from CFD data collected for the SFPW at six angles of attack $\left(0-25^{\circ}\right)$ at three typical surfing velocities ( $\mathrm{U}_{\infty}=3 \mathrm{mps}, 5 \mathrm{mps}$, and $7 \mathrm{mps}$ )

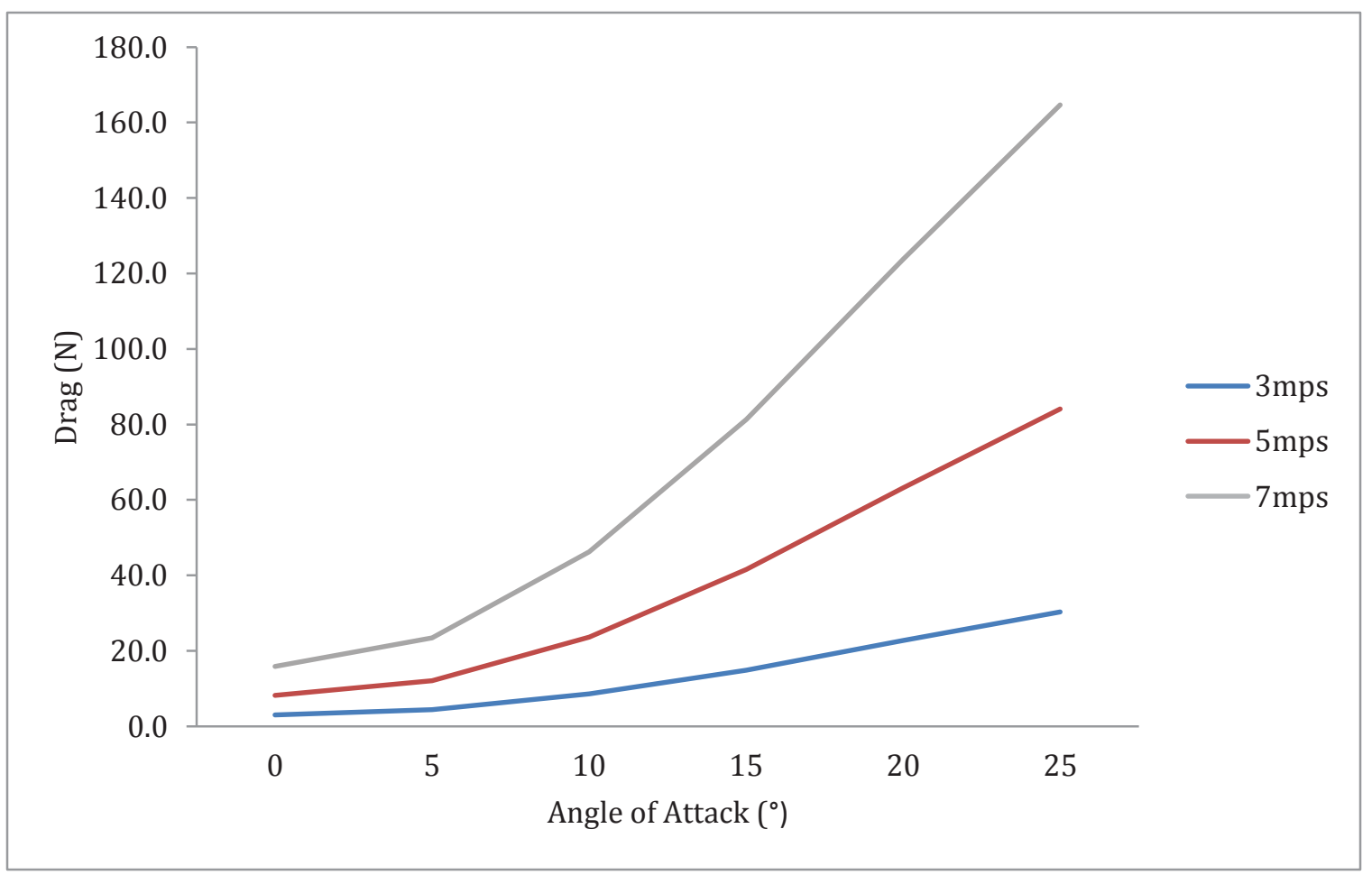

Figure 26: Drag (N) from CFD data collected for the SFPW at six angles of attack (0$25^{\circ}$ ) at three typical surfing velocities ( $\mathrm{U}_{\infty}=3 \mathrm{mps}, 5 \mathrm{mps}$, and $7 \mathrm{mps}$ ) 
Figure 26 presents the drag force data collected for the three high velocity simulations. At the higher velocities, drag force vales were greater. Drag force grew in value as the angle of attack intensified. The SFPW had the highest drag force $(164.7 \mathrm{~N})$ at $25^{\circ}$ angle of attack with an upstream velocity of $7 \mathrm{mps}$. At $3 \mathrm{mps}$, the highest drag value was $30.3 \mathrm{~N}$ at $25^{\circ}$ angle of attack. For $5 \mathrm{mps}$, the drag force value was $84.1 \mathrm{~N}$ at this same angle.

Table 14: Lift $(\mathrm{N})$ and drag $(\mathrm{N})$ forces from CFD data collected for the SFPW at six angles of attack $\left(0-25^{\circ}\right)$ at three typical surfing velocities $\left(\mathrm{U}_{\infty}=3 \mathrm{mps}, 5 \mathrm{mps}\right.$, and $7 \mathrm{mps}$ )

\begin{tabular}{|c|c|c|c|c|c|}
\hline & $A O A$ & Lift (N) & Drag (N) & $C_{L}$ & $C_{D}$ \\
\hline \multirow{6}{*}{ 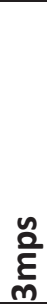 } & 0 & 0.1 & 3.0 & 0.00 & 0.05 \\
\hline & 5 & 20.7 & 4.4 & 0.36 & 0.08 \\
\hline & 10 & 40.0 & 8.6 & 0.70 & 0.15 \\
\hline & 15 & 54.8 & 14.9 & 0.96 & 0.26 \\
\hline & 20 & 65.8 & 22.8 & 1.15 & 0.40 \\
\hline & 25 & 69.4 & 30.3 & 1.21 & 0.53 \\
\hline \multirow{6}{*}{ 气ू } & 0 & 0.3 & 8.1 & 0.00 & 0.05 \\
\hline & 5 & 57.5 & 12.0 & 0.36 & 0.08 \\
\hline & 10 & 111.2 & 23.7 & 0.70 & 0.15 \\
\hline & 15 & 153.2 & 41.5 & 0.96 & 0.26 \\
\hline & 20 & 182.8 & 63.2 & 1.15 & 0.40 \\
\hline & 25 & 192.7 & 84.1 & 1.21 & 0.53 \\
\hline \multirow{6}{*}{ 气̆ } & 0 & 0.6 & 15.8 & 0.00 & 0.05 \\
\hline & 5 & 112.7 & 23.4 & 0.36 & 0.08 \\
\hline & 10 & 218.0 & 46.2 & 0.70 & 0.15 \\
\hline & 15 & 300.4 & 81.3 & 0.96 & 0.26 \\
\hline & 20 & 358.3 & 123.7 & 1.15 & 0.40 \\
\hline & 25 & 377.6 & 164.7 & 1.21 & 0.53 \\
\hline
\end{tabular}

The quantitative lift and drag force results for the high velocity experiments are presented in Table 14, along with the associated lift and drag coefficient values. The lift and drag coefficients were almost identical between the three velocities. There were extremely small differences between the coefficient values. There was less than a $1 \%$ difference between the three velocities for lift coefficient values, except at $0^{\circ}$ angle of attack. At this angle, there was about a $2 \%$ difference. Comparing the lift coefficients for the high velocity results to the CFD $0.5 \mathrm{mps}$ lift coefficient results, there was less than a $1 \%$ difference, 
except at $0^{\circ}$ angle of attack. At this angle, there was a value difference between the $0.5 \mathrm{mps}$ lift coefficient results and the high velocity results of only 0.0001 , but an average percent difference of $5.7 \%$.

Similar percent differences were seen for the drag coefficients between the high angles of attack, except at $0^{\circ}$ and $5^{\circ}$ angle of attack the average difference was slightly over $1 \%$. Comparing the high velocity drag coefficient values to the CFD $0.5 \mathrm{mps}$ drag coefficient results, there was found to be greater discrepancies than the lift coefficients. There was less than a $1 \%$ difference from $20^{\circ}$ to $25^{\circ}$. At $10^{\circ}$ and $15^{\circ}$, the variance ranged from $2.5 \%$ to $5.4 \%$. From $0^{\circ}$ to $5^{\circ}$, the average percent difference was $12.5 \%$, with a high of $15.8 \%$ difference at $0^{\circ}$ between the drag coefficients of $7 \mathrm{mps}$ and $0.5 \mathrm{mps}$. As the velocities increased, there was a larger variance from the lift and drag results at $0.5 \mathrm{mps}$.

When predicting the lift and drag forces at these higher angles of attack, using Equation 7 and Equation 8, as well as the coefficient results from the CFD $0.5 \mathrm{mps}$ values, there was found to be a small range of error. For the lift forces there was less than a $1 \%$ difference between the CFD results and predicted lift force results for $3 \mathrm{mps}, 5 \mathrm{mps}$, and $7 \mathrm{mps}$; this excluded $0^{\circ}$ angle of attack. At $0^{\circ}$ incident angle, the greatest difference in value was $0.05 \mathrm{~N}$ at $7 \mathrm{mps}$. This provided a $7.2 \%$ difference between the predicted values at $7 \mathrm{mps}$ and the CFD results at $7 \mathrm{mps}$. The drag forces generated a higher range of error. From $20^{\circ}$ to $25^{\circ}$, the difference between the predicted drag forces and the CFD drag forces was less than $1 \%$ for $3 \mathrm{mps}, 5 \mathrm{mps}$, and $7 \mathrm{mps}$. At $15^{\circ}$, there was about a $2.7 \%$ difference between predicted and CFD drag forces for the three high velocities, and about a 5.1\% difference at $10^{\circ}$. There was approximately $10.2 \%$ of a difference at $5^{\circ}$ and a $14.7 \%$ difference at $0^{\circ}$. The greatest difference found was at $0^{\circ}$ for $7 \mathrm{mps}$, yielding a $15.8 \%$ difference $(2.72 \mathrm{~N})$.

Figure 27 shows the three high velocity L/D ratio curves. It can be seen that the curves are extremely close in shape and L/D ratio values. For the three high velocity values presented, all $\mathrm{L} / \mathrm{D}$ ratios peak at $5^{\circ}$ angle of attack; this is congruent with the $0.5 \mathrm{mps} \mathrm{L} / \mathrm{D}$ ratio results for the SFPW. The greatest difference between the three high velocities was found at this peak incident angle, causing a $1.71 \%$ variance between $3 \mathrm{mps}$ and $7 \mathrm{mps}$. Comparing the $\mathrm{L} / \mathrm{D}$ ratios for the high velocity values to the $0.5 \mathrm{mps}$ results, the greatest difference was 
produced from the results at $7 \mathrm{mps}$. The greatest variance was at $5^{\circ}$ angle of attack (11.66\%), but the $\mathrm{L} / \mathrm{D}$ ratio value difference was only 0.53 .

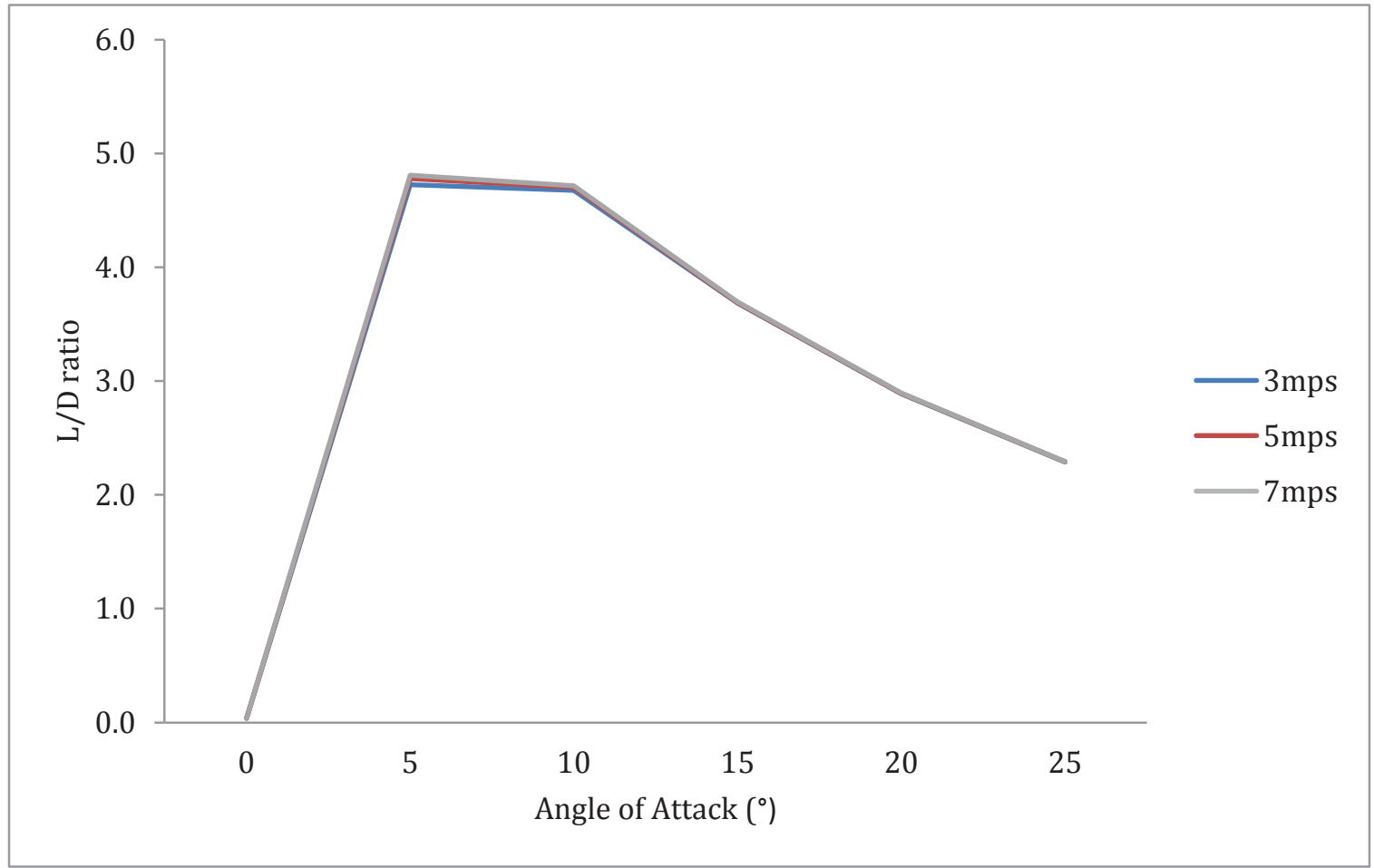

Figure 27: $\mathrm{L} / \mathrm{D}$ ratio from CFD data collected for the SFPW at six angles of attack (0$\left.25^{\circ}\right)$ at three typical surfing velocities $\left(\mathrm{U}_{\infty}=3 \mathrm{mps}, 5 \mathrm{mps}\right.$, and $\left.7 \mathrm{mps}\right)$ 


\subsubsection{FLOW VISUALIZATION OF THE CFD RESULTS}

The visual portion of this thesis presents images from the CFD results to give flow field representations of the numerical data results. The direction and magnitude of the flow around the SFPW fin was analyzed. This provided an understanding of the reaction of the flow field as the optimum fin was repositioned at various angles of attack. The CFD images were compared to photo images collected from the experiments in the water channel. Four incident angles were selected to present and discuss within the report: $0^{\circ}$, $10^{\circ}, 25^{\circ}, 45^{\circ}$. At $0^{\circ}$ incident angle, the fin was most aerodynamic and can be used as the control variable in order to compare flow fields at higher angles of attack. Referring back to Figure $3,10^{\circ}$ incident angle was chosen because the L/D ratio peaked here. The critical angle of attack, for the lift forces and coefficients, was found at $25^{\circ}$ and the drag values were highest at $45^{\circ}$.

Figure 28 presents the velocity magnitudes and flow field around the SFPW at $0^{\circ}$ angle of attack. At $0^{\circ}$, water flow occurs symmetrically on both sides of the hydrofoil. Illustrated in Figure 28(B), the streamline shape allowed the fluid to move smoothly over the surface of the fin, with very little disruption in the surrounding flow regime. Velocity and pressures changed across the surface of the fin. As anticipated, at the leading edge, velocity decreased due to the oncoming water's reaction to the blunt edge of the fin; Figure 28(A). This caused an increase in pressure at the leading edge as shown in Figure 29. The yellow isolines display the positive static pressure on the surface of the fin's leading edge. The light blue to dark blue coloration represents a decrease in velocity at the leading edge, ranging from 0.32 to $0.41 \mathrm{mps}$. As the fluid moved to either side of the symmetric shape, velocity increased from beyond the normal upstream velocity of $0.5 \mathrm{mp}$ to upwards of $0.57 \mathrm{mps}$. This was a result of the Bernoulli's effect. The swelling width of the hydrofoil's camber caused the contraction of the streamlines, as shown in Figure $28(\mathrm{C})$. This caused a simultaneous decrease in pressure and increase in velocity. At the sharp trailing edge of the fin, pressure once again increased and velocity decreased. The flow vectors and streamlines are represented in a two-dimensional sheet (YC-XC). 


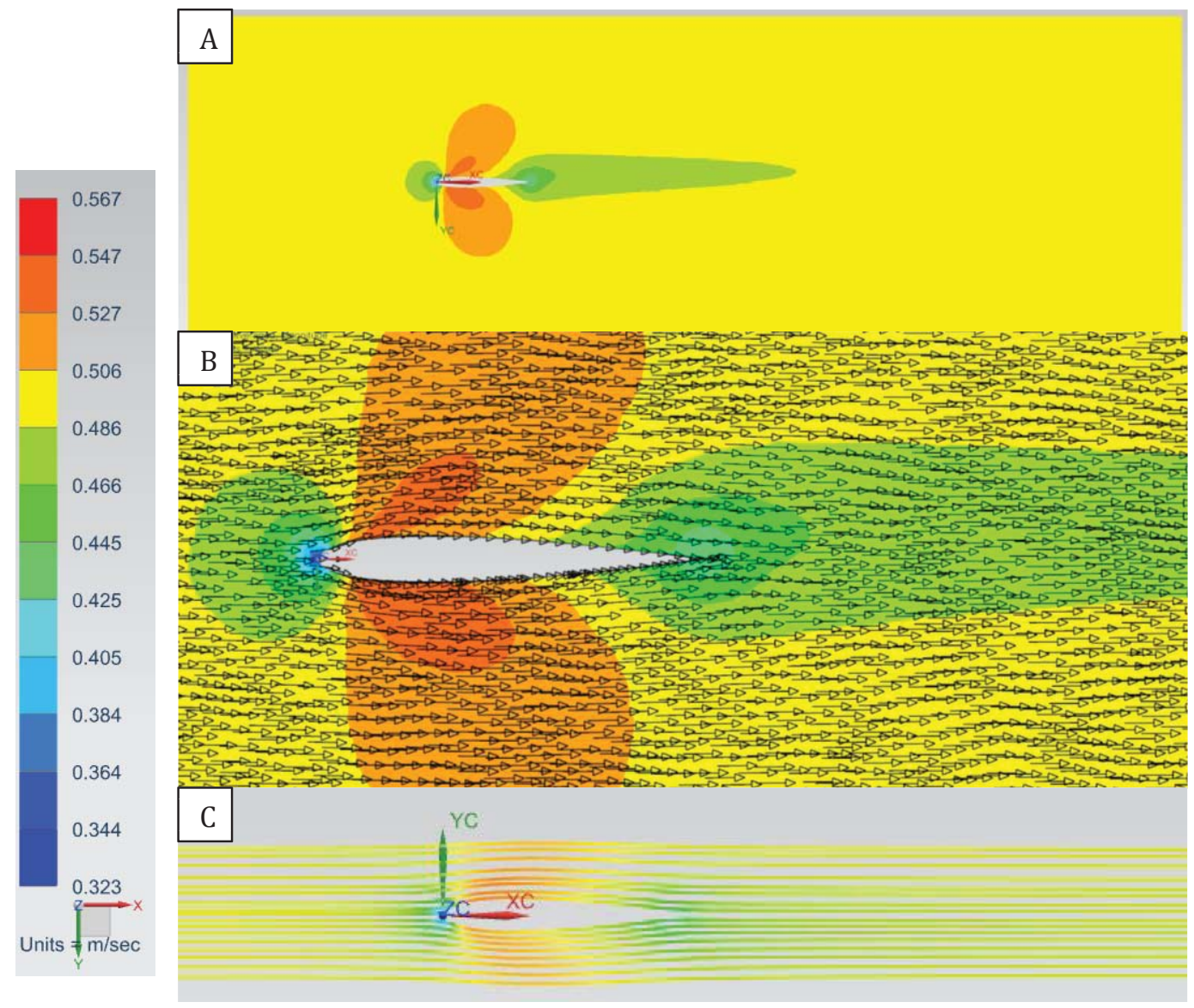

Figure 28: Plan view at the base section of the SFPW at $\alpha=0^{\circ}, \mathrm{U}_{\infty}=0.5 \mathrm{mps}$ (A) Velocity magnitudes. (B) Velocity magnitudes and flow vectors. (C) Streamlines
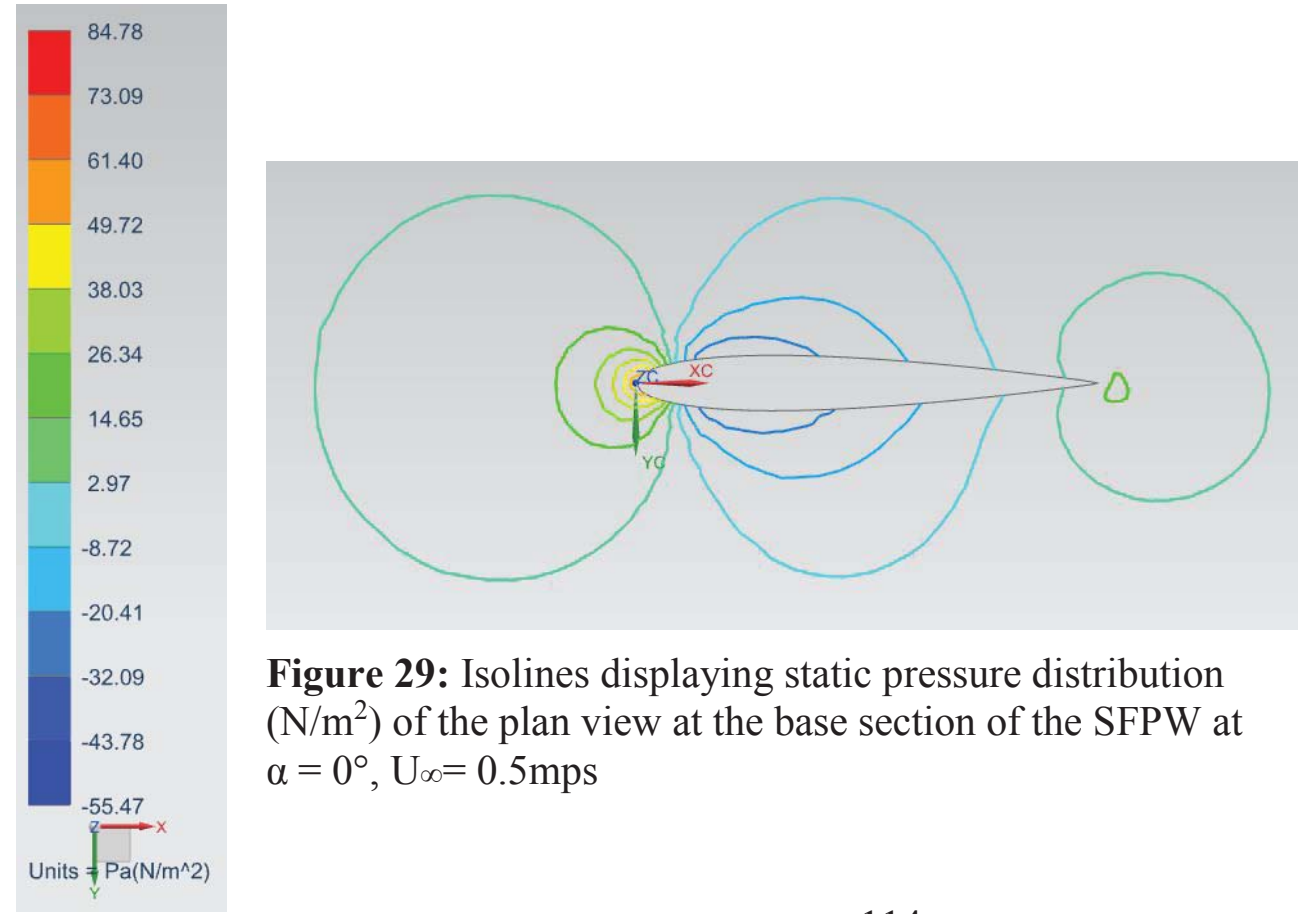

Figure 29: Isolines displaying static pressure distribution $\left(\mathrm{N} / \mathrm{m}^{2}\right)$ of the plan view at the base section of the SFPW at $\alpha=0^{\circ}, \mathrm{U}_{\infty}=0.5 \mathrm{mps}$ 
Similar flow mannerisms were found at the mid-section of the fin, as shown in Figure 30(A). This figure also provides the flow vectors in a two-dimensional sheet (YC-XC). At the stagnation point of the leading edge, pressure increased and velocity decreased. Pressure and velocity distribution was not as severe near the mid-section, as compared to the base of the fin. Velocity increased at the widest point on either side of the fin, and then decreased at the trailing edge.

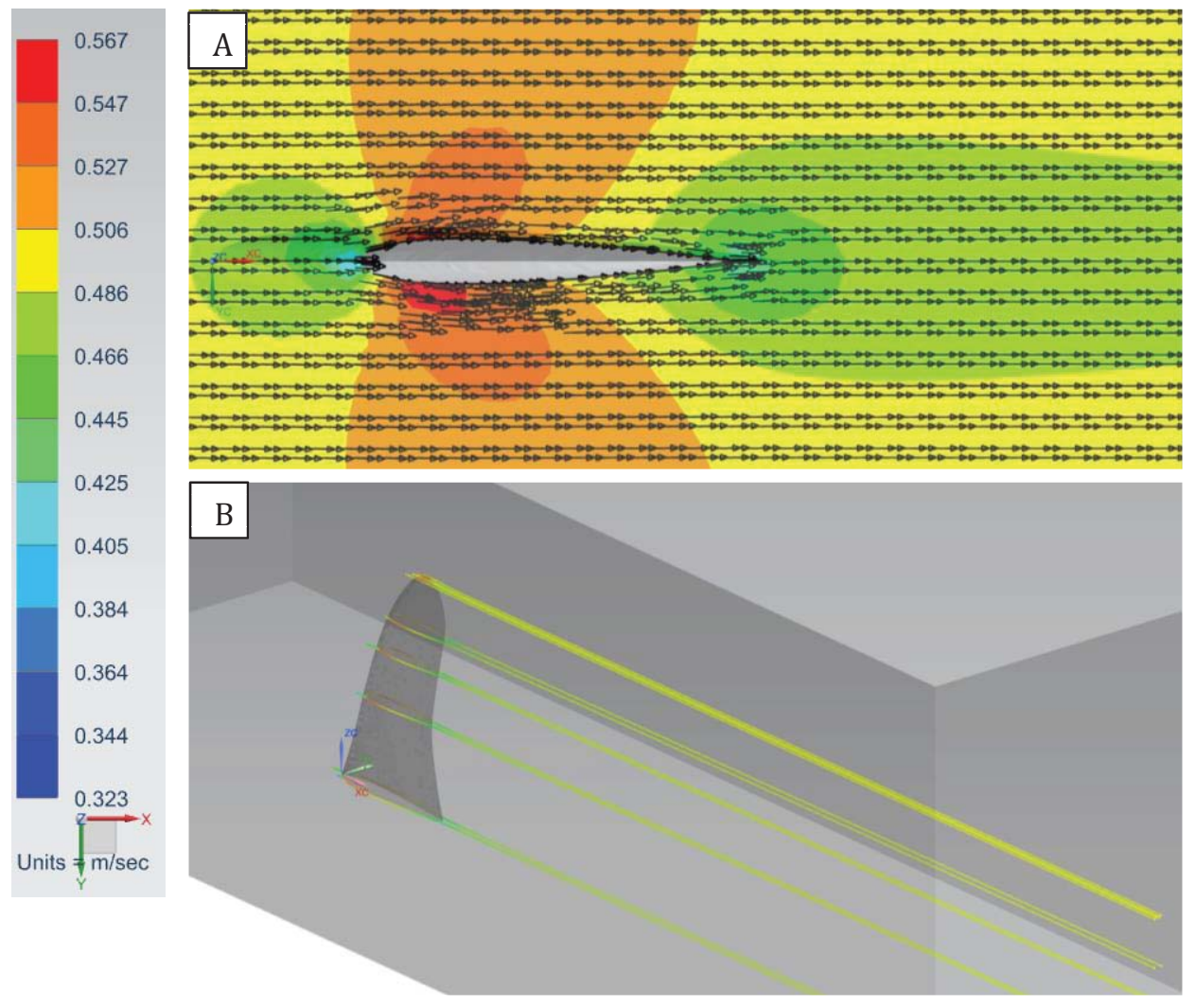

Figure 30: SFPW at $\alpha=0^{\circ}, \mathrm{U}_{\infty}=0.5 \mathrm{mps}(A)$ Velocity magnitudes and flow vectors, plan view at the mid-section (B) Streamlines, isometric view from trailing edge of the fin

A three-dimensional isometric view, looking towards the trailing edge, is shown in Figure 30(B). This image illustrates the streamlines around the SFPW at five different locations along the length of the fin. The base, three mid-section locations, and the tip of the fin were seeded for streamline locations. Due to the $0^{\circ}$ angle of attack, the streamlines showed very little disruption in the direction of flow downstream. The direction of flow was shown to move from the upper left hand corner of the image to the lower right 
hand corner. The green color at the leading edge showed the decrease in velocity, but moved to a red-orange color near the sides of the fin. Velocity increased at the tip of the fin, shown in a red-orange color. At the trailing edge, the velocity magnitudes were shown as green, which depicted a decrease in velocity. Moving downstream, the streamlines transitioned to a yellow color, meaning free-stream velocity was restored.

Figure 31 and Figure 32 present the SFPW at $0^{\circ}$, in the profile position. The velocity magnitudes and flow vectors are shown in two-dimensional form in order to understand the flow three-dimensionally. At the leading edge, flow velocity decreased and was more significant towards the base of the fin. Moving towards the tip of the fin, velocity increased and was greatest at the tip. In Figure 32, velocity increases at the side of the fin, along the length. High velocities are more substantial at the base to mid-section of the SFPW fin. Velocity decreases as flow moves from the sides of the fin towards the trailing edge. The fin caused very little disruption in the flow regime as the flow direction moved horizontally in the direction of flow.

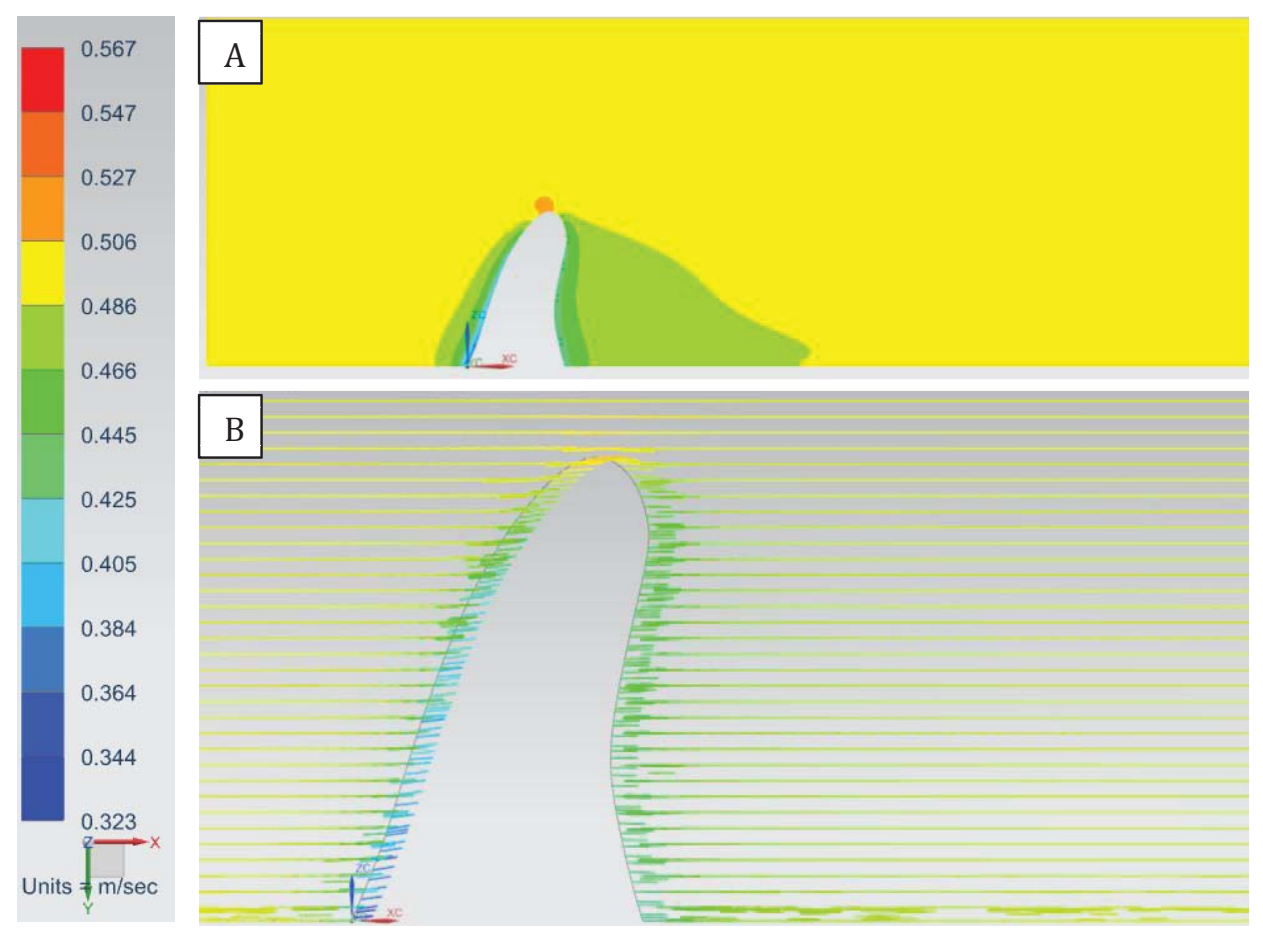

Figure 31: Profile view at the leading-edge section of the SFPW at $\alpha=0^{\circ}, \mathrm{U}_{\infty}=0.5 \mathrm{mps}$ (A) Velocity magnitudes (B) Flow vectors 


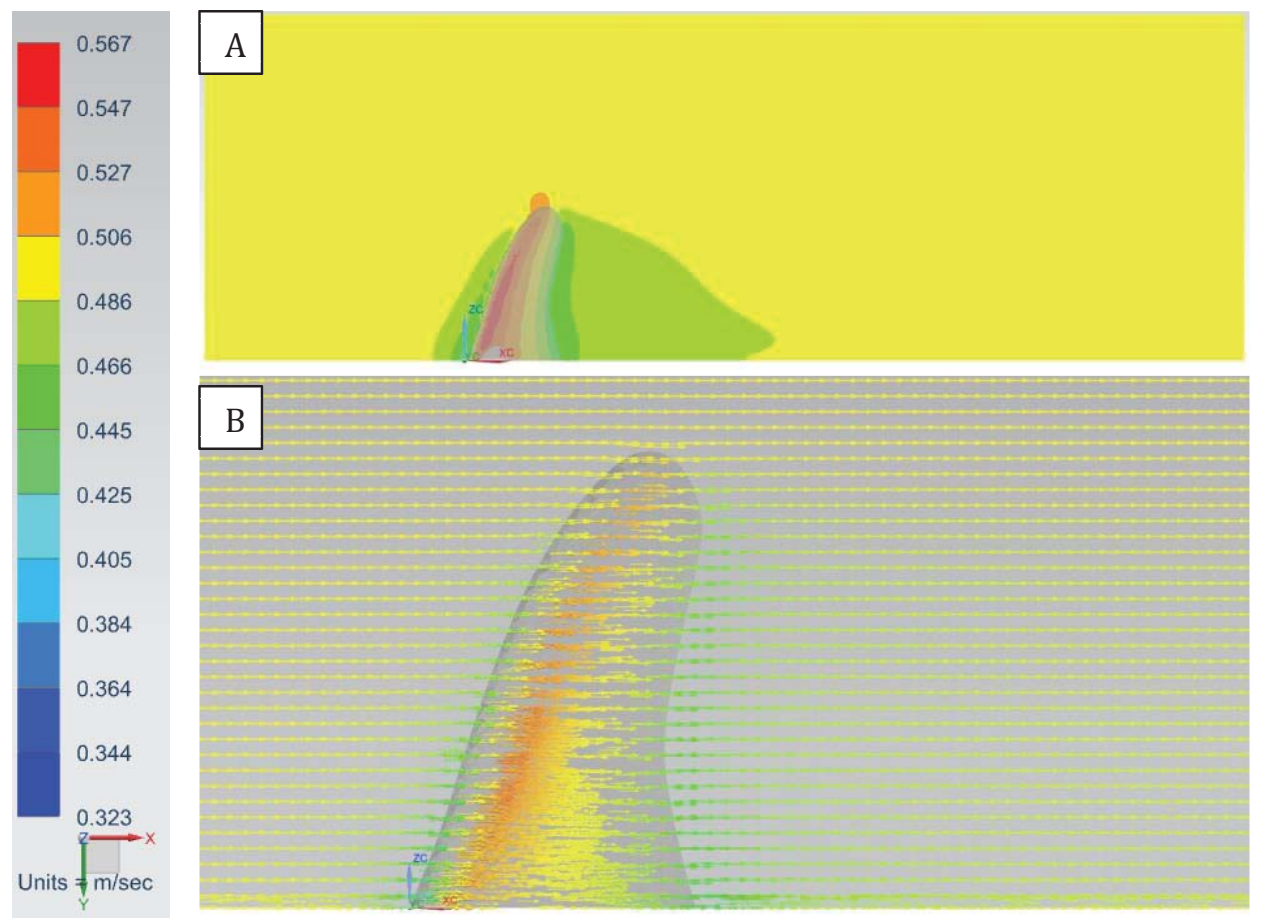

Figure 32: Profile view at the upper camber surface section of the SFPW at $\alpha=0^{\circ}$, $\mathrm{U}_{\infty}=0.5 \mathrm{mps}(A)$ Flow vectors $(B)$ Velocity magnitudes

At a small angle of attack of $10^{\circ}$, pressure and velocity distributions shifted. At the leading edge, the decrease in velocity moved towards the bottom surface of the fin. This was because the change in incident angle caused that area of the fin to receive the initial blunt force of the oncoming flow. The dark blue area displays a significant decrease in velocity. Speeds as low as $0.22 \mathrm{mps}$ were recorded at the leading edge on the compression side, as shown in Figure 33(A). On the opposing suction side at the leading edge, velocity increased to 0.54 to $0.64 \mathrm{mps}$. At the trailing edge, there was also a decrease in velocity on the suction side. The velocity decrease was not as significant as the leading edge, only dropping to approximately 0.36 to $0.39 \mathrm{mps}$. 


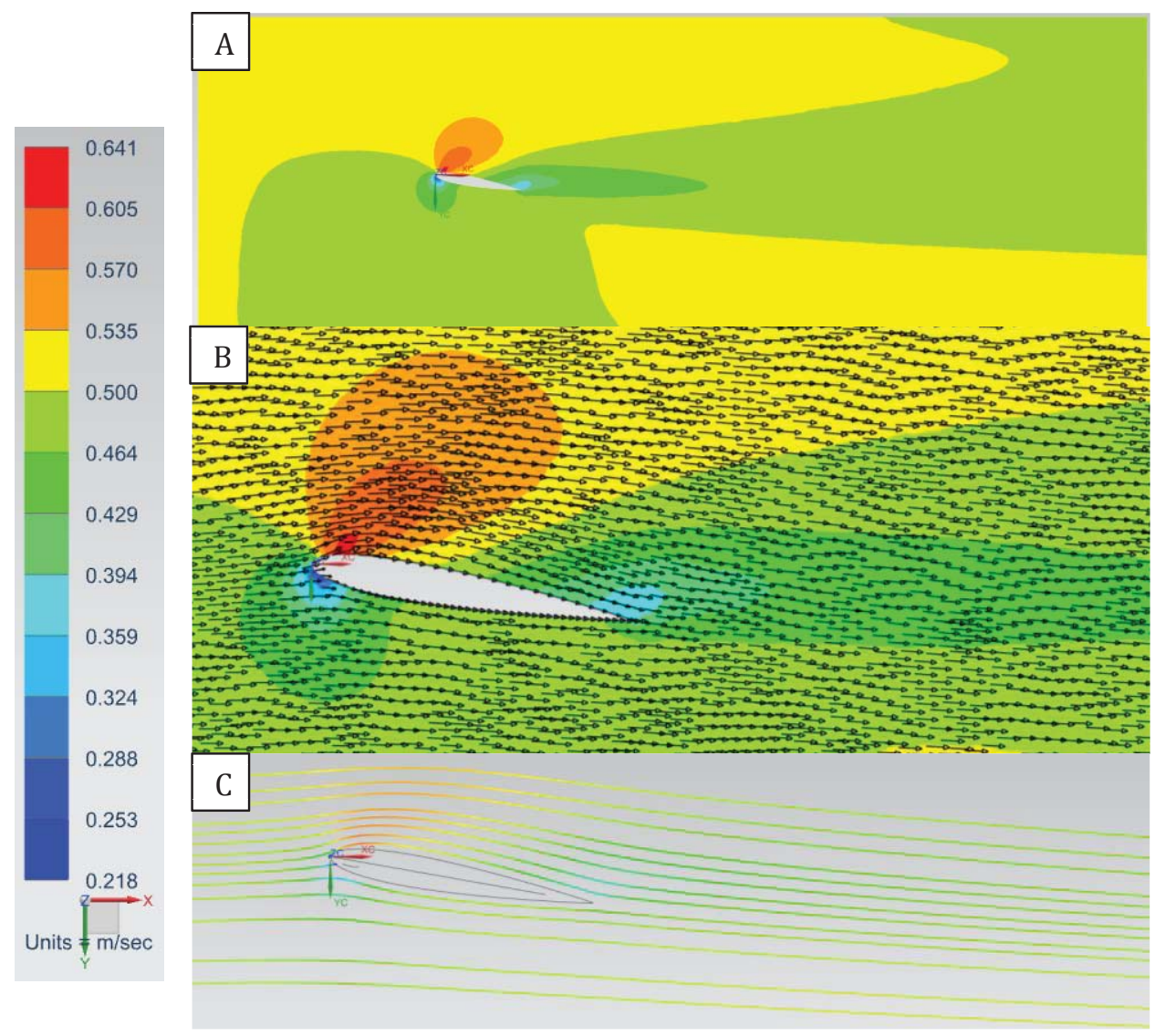

Figure 33: Plan view at the base section of the SFPW at $\alpha=10^{\circ}, \mathrm{U}_{\infty}=0.5 \mathrm{mps}$ (A) Velocity magnitudes. (B) Velocity magnitudes and flow vectors. (C) Streamlines

Figure 33(B) and Figure 33(C) provides the flow vector and streamline visual information, respectively. At $10^{\circ}$, the hydrofoil did not substantially impact the structure of the flow. The fluid moves smoothly around the surface of the fin without any signs of flow separation. The direction of flow generally moves horizontally, with the exception of a slight change in angle near the fins boundary. This is depicted in Figure 33(C). At the top surface, near the leading edge, the streamlines compress causing the increase in velocity and decrease in pressure. The negative pressure values are shown in blue on the top surface of the fin in Figure 34. There was an equal, yet opposite expansion effect on the bottom surface. The streamlines follow the curve shape of the hydrofoil, impacting the flow direction around the fin. 

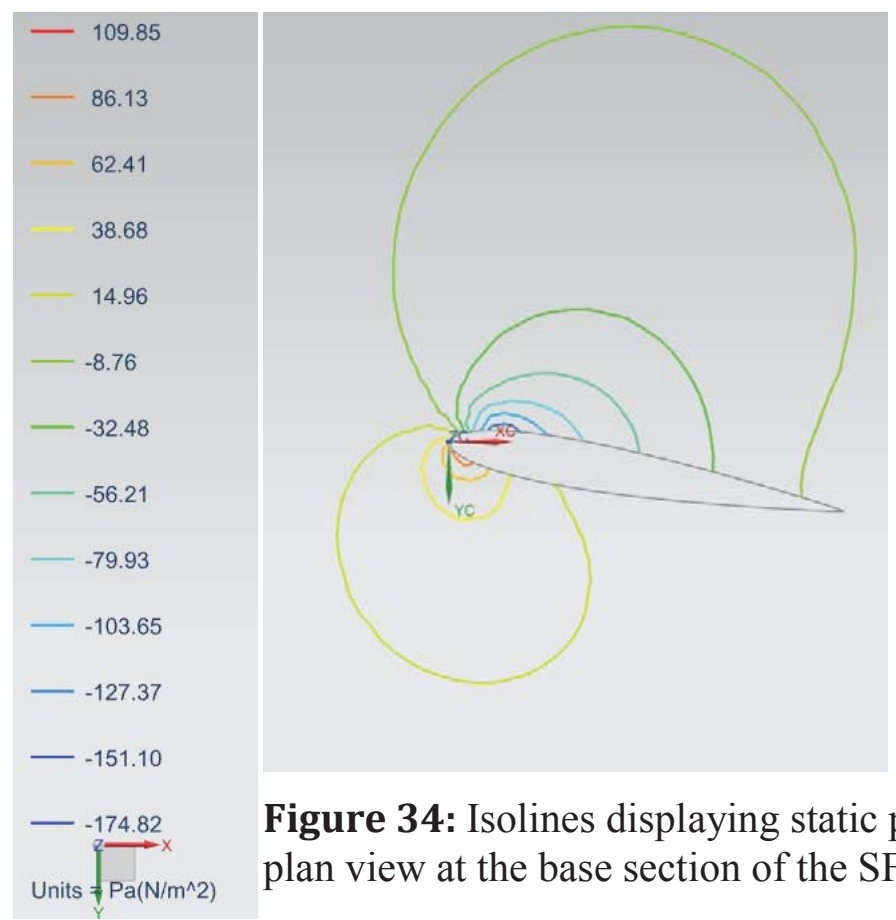

Figure 34: Isolines displaying static pressure distribution $\left(\mathrm{N} / \mathrm{m}^{2}\right)$ of the plan view at the base section of the SFPW at $\alpha=10^{\circ}, \mathrm{U}_{\infty}=0.5 \mathrm{mps}$

The direction of flow near the surface of the fin is again shown in Figure 35(A) for the mid-section of the SFPW at $10^{\circ}$ angle of attack. At the leading edge, velocity increased on the upper surface and decreased on the bottom surface. The flow compressed while moving around the upper stagnant region of the fin. The fluid also changed direction in order to move around the upper surface. At the upper surface of the trailing edge, velocity decreased. Figure 35(B) shows the streamlines around the entire fin. The position of the SFPW fin induced marginal effects on the direction of flow in the downstream wake. At the tip of the fin, tip vortices were starting to develop causing some downstream disturbance. These tip vortices moved in the counter-clockwise direction, when viewed looking from the downstream direction at the trailing edge. 


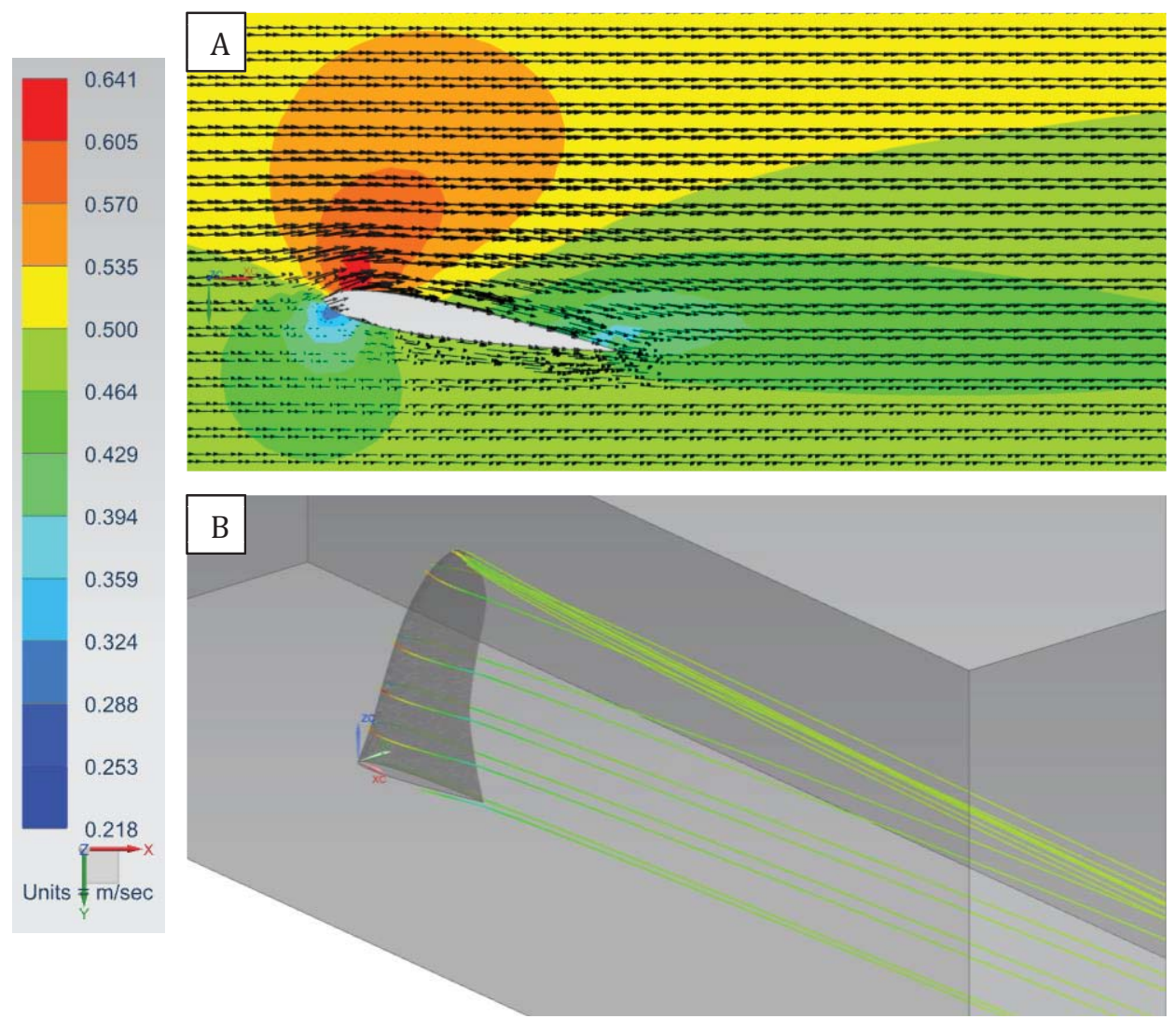

Figure 35: SFPW at $\alpha=10^{\circ}$, $\mathrm{U}_{\infty}=0.5 \mathrm{mps}$ (A) Velocity magnitudes and flow vectors, plan view at the mid-section (B) Streamlines, isometric view from trailing edge of the fin 
Figure 36 through Figure 38 show the flow across the profile of the fin. The flow vectors are represented in a two-dimensional plane ( $\mathrm{ZC}-\mathrm{XC})$. The leading edge of the compression surface shows an increase in velocity along the length of the fin. The escalation in velocity up to $0.64 \mathrm{mps}$ can be seen in Figure 36(B). Figure 36(A) shows that the flow direction does not change from the normal horizontal flow path. Flow movement was shown to compress closer together near the leading edge.
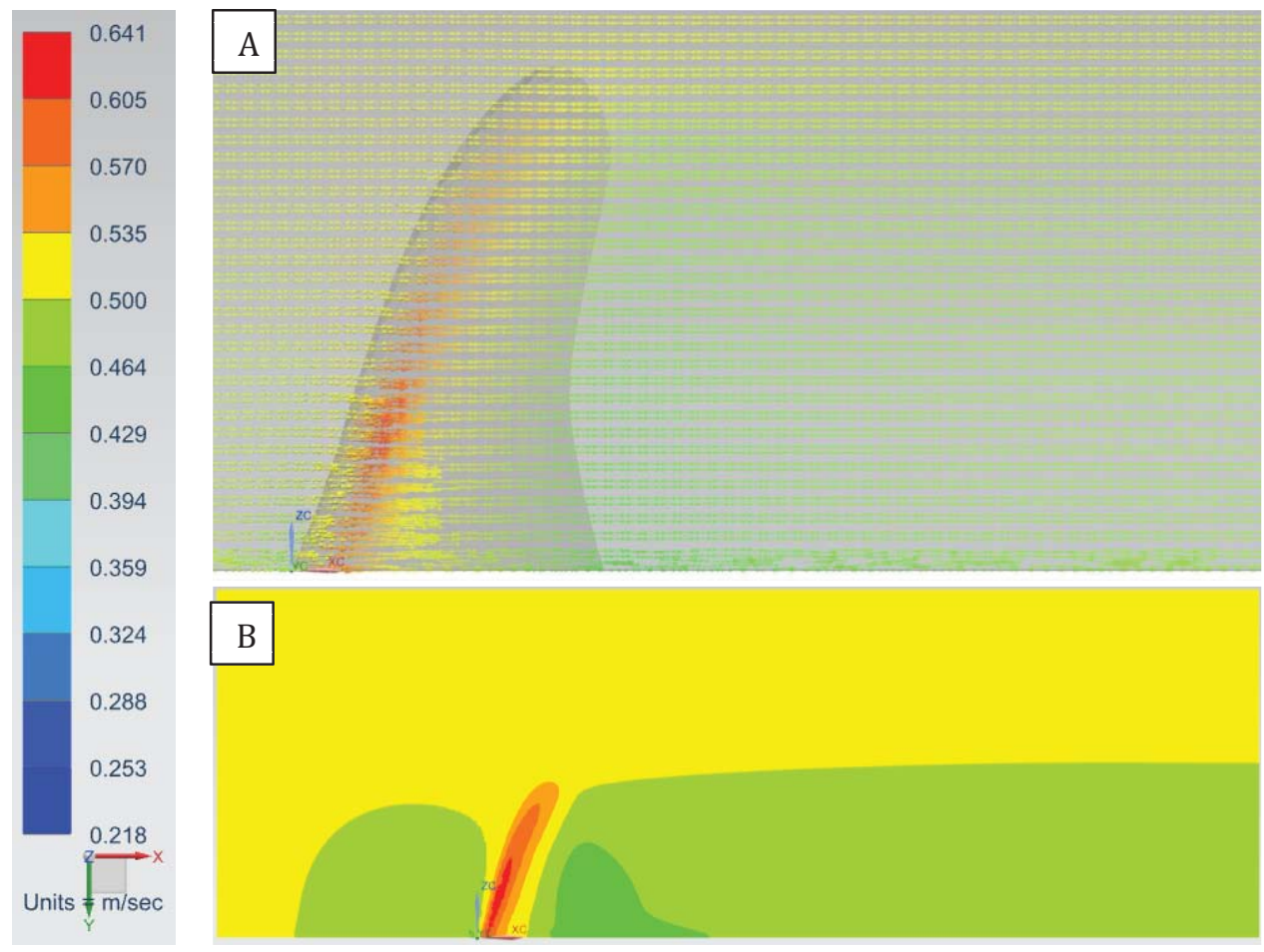

Figure 36: Profile view at the leading-edge section of the SFPW at $\alpha=10^{\circ}, \mathrm{U}_{\infty}=$ $0.5 \mathrm{mps}$ (A) Flow vectors (B) Velocity magnitudes

In Figure 37, the profile view of the middle-edge section is shown, looking at the suction side. Because the vectors are represented two-dimensionally, the flow intersected with the fin on the compression side, and on the suction side after traversing the mid-section of the fin. There was very little change in velocity magnitudes or flow direction in this section. Velocity values marginally decreased on both sides of the fin, as shown in Figure 37 (A). On the suction side, values decreased to about 0.36 to $0.39 \mathrm{mps}$ for the entire length of the fin. There was a small change in the angle of the flow direction as the 
upstream flow came in contact with the fin on the compression side. This was especially true in the upper region of the fin as the flow slightly changed angle as it moved upwards towards the tip, as shown in Figure 37(B). The upper region is the area towards the tip of the fin and the lower region is at the base of the fin.
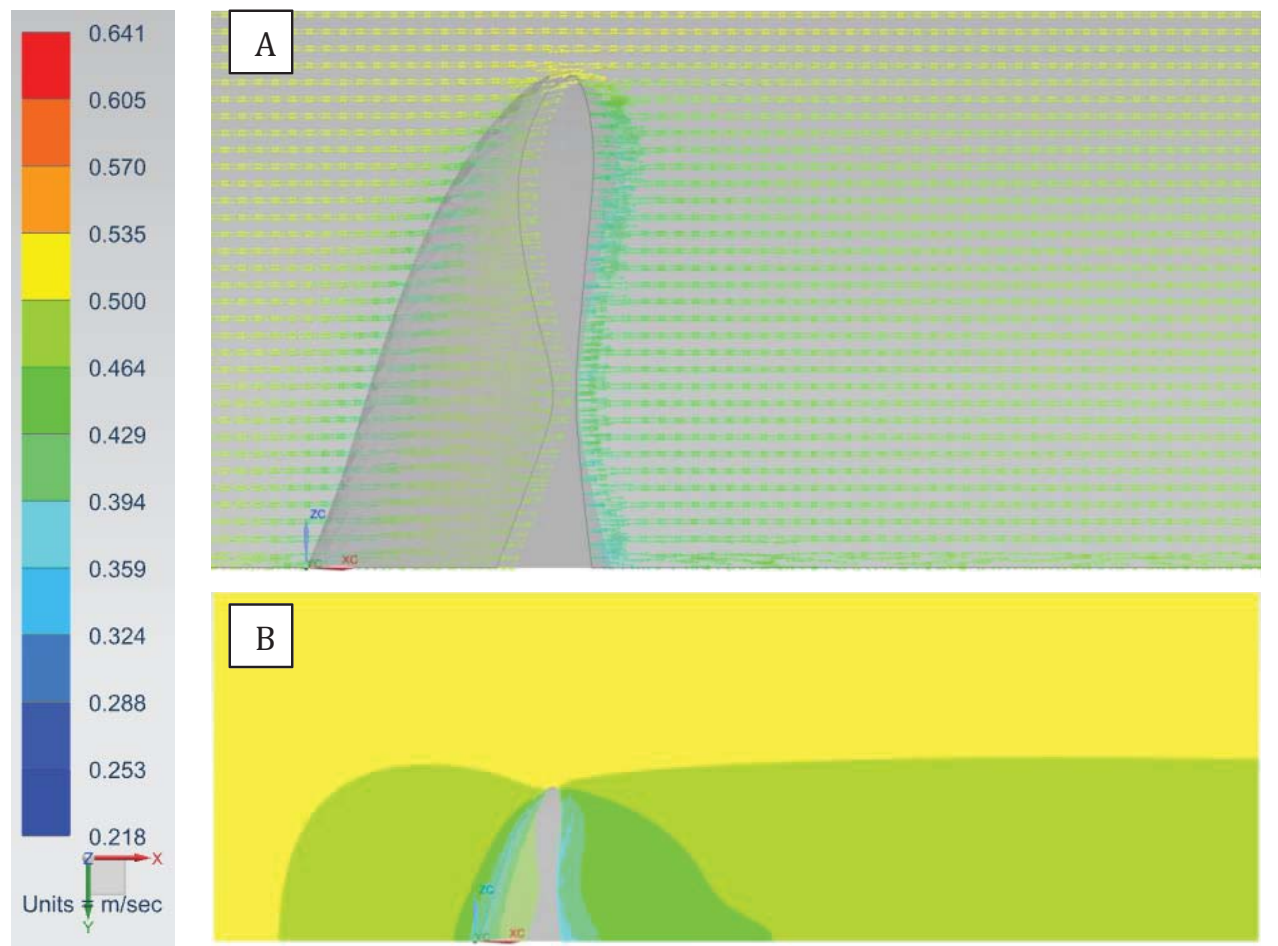

Figure 37: Profile view at the mid-edge section of the SFPW at $\alpha=10^{\circ}, \mathrm{U}_{\infty}=0.5 \mathrm{mps}$ (A) Flow vectors (B) Velocity magnitudes

The profile view of the trailing edge is shown in Figure 38. Again, there was only a small change in velocity magnitudes and flow direction. The oncoming flow increased in angle while coming in contact with the fin, moving towards the fin tip. This was shown in Figure 38(A). This was predominantly true for the upper region of the fin. There was very little change in velocity on the suction side of the fin, but the velocity decreased after the flow had passed over the trailing edge. Values dipped below $0.4 \mathrm{mps}$ along the length of the fin after the trailing edge section; Figure 38(B). There was very little change in the horizontal direction of the flow downstream of the trailing edge. 

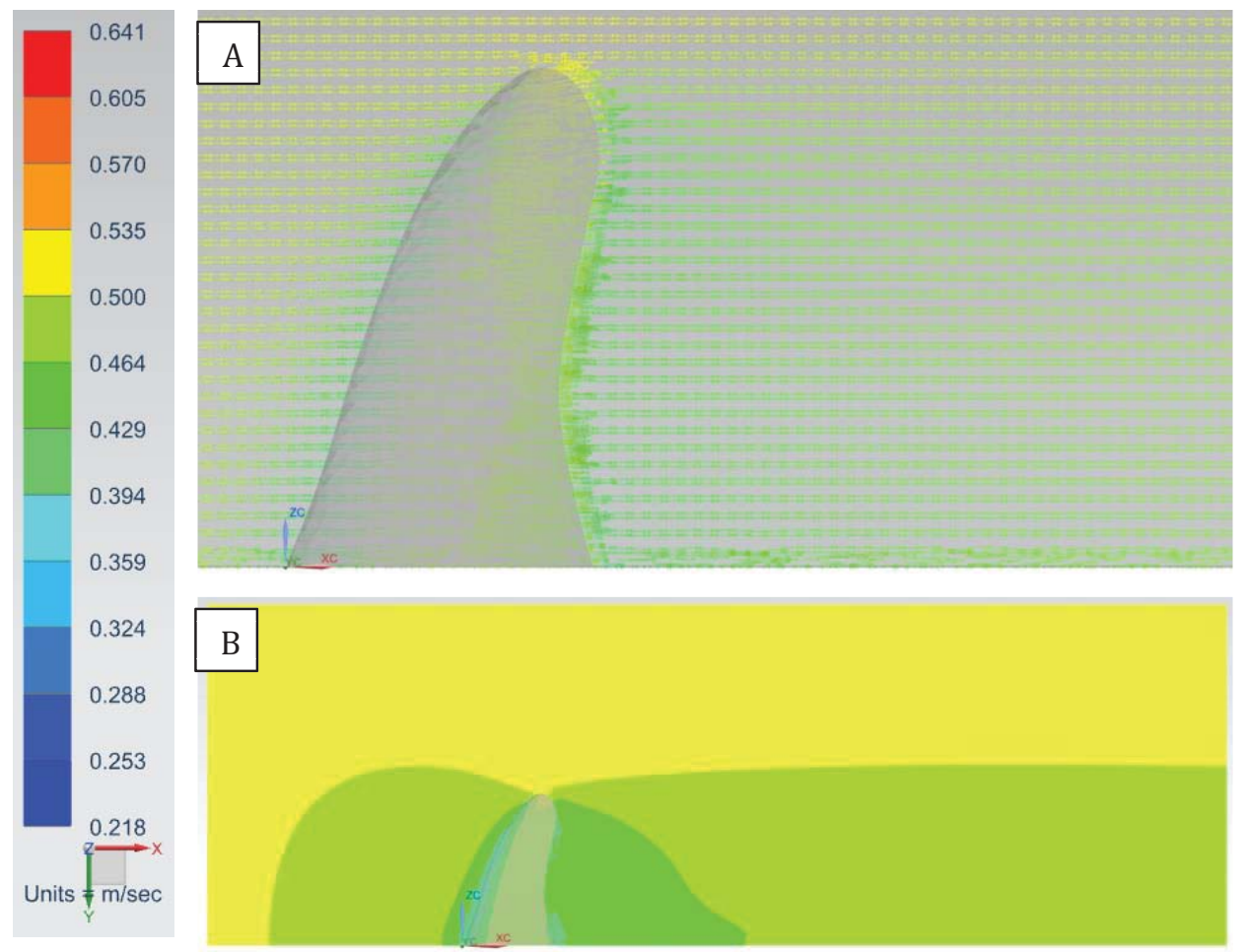

Figure 38: Profile view at the trailing-edge section of the SFPW at $\alpha=10^{\circ}, \mathrm{U}_{\infty}=0.5 \mathrm{mps}$ (A) Flow vectors (B) Velocity magnitudes

At the lift coefficient's critical angle of attack of $25^{\circ}$, velocity magnitudes and the movement of flow were analyzed. Figure 39 represents the plan view of the base section for the SFPW at $25^{\circ}$. As compared to the smaller angles of attack, the shifting of the velocity and pressure gradients intensified at $25^{\circ}$. The velocity magnitude values also amplified as the direction of flow was altered. In Figure 39(A), the stagnation point repositioned even farther from the fin's leading edge, compared to the location at $10^{\circ}$ angle of attack. On the bottom surface, the decrease in velocity moved slightly farther away from the tip of the hydrofoil. The values decreased to as low as $0.05 \mathrm{mps}$ and affected a wider region outside of the flow boundary of the fin compared to the $10^{\circ}$ angle of attack. The pressure increased in this area, as shown in a red to orange coloration in Figure 40. The opposing upper surface high velocity region shifted in position towards the leading edge. Values increased to as high as $0.69 \mathrm{mps}$ and encompassed a larger affected region. Associated negative pressure values were shown in light blue to dark blue coloration in Figure 40. The most significant change in velocity magnitudes and 
regions affected was found at the trailing edge of the compression surface. This area of decreased velocity extends from the trailing edge to beyond the mid-edge section of the fin.

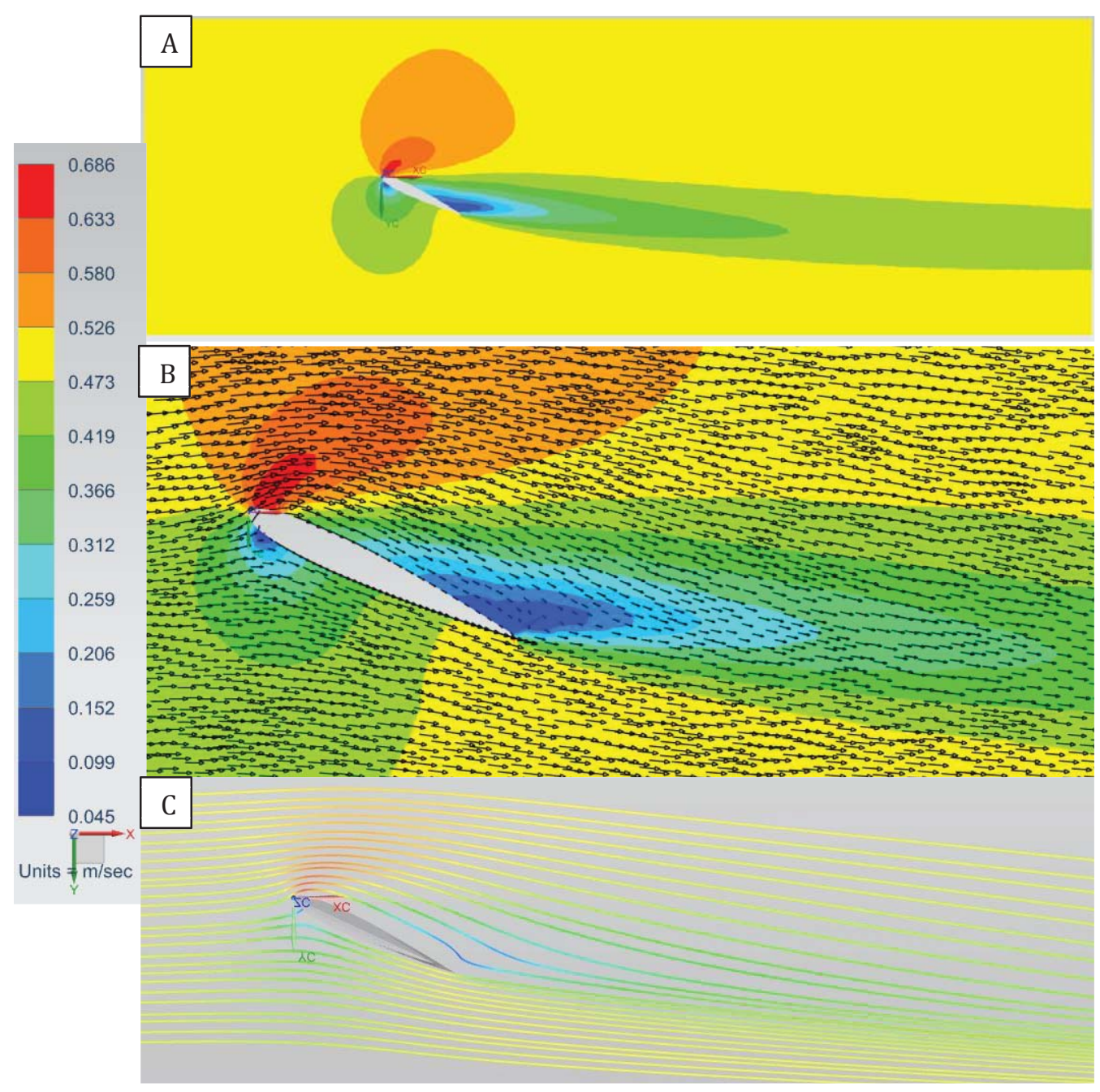

Figure 39: Plan view at the base section of the SFPW at $\alpha=25^{\circ}, \mathrm{U}_{\infty}=0.5 \mathrm{mps}$ (A) Velocity magnitudes. (B) Velocity magnitudes and flow vectors. (C) Streamlines

The flow vectors and streamline images are shown in Figure 39(B) and Figure 39(C), respectively. Within the stagnation region, flow velocities decreased substantially. As the fluid moved around the upper surface, velocity increased and the directions of flow changed. The streamlines mimicked the shape of the hydrofoil surface, which caused the small disruption in flow direction. Near the trailing edge of the compression side, there 
was a substantial decrease in velocity. The streamlines in this region did not flow horizontally, but followed the shape of the hydrofoil.
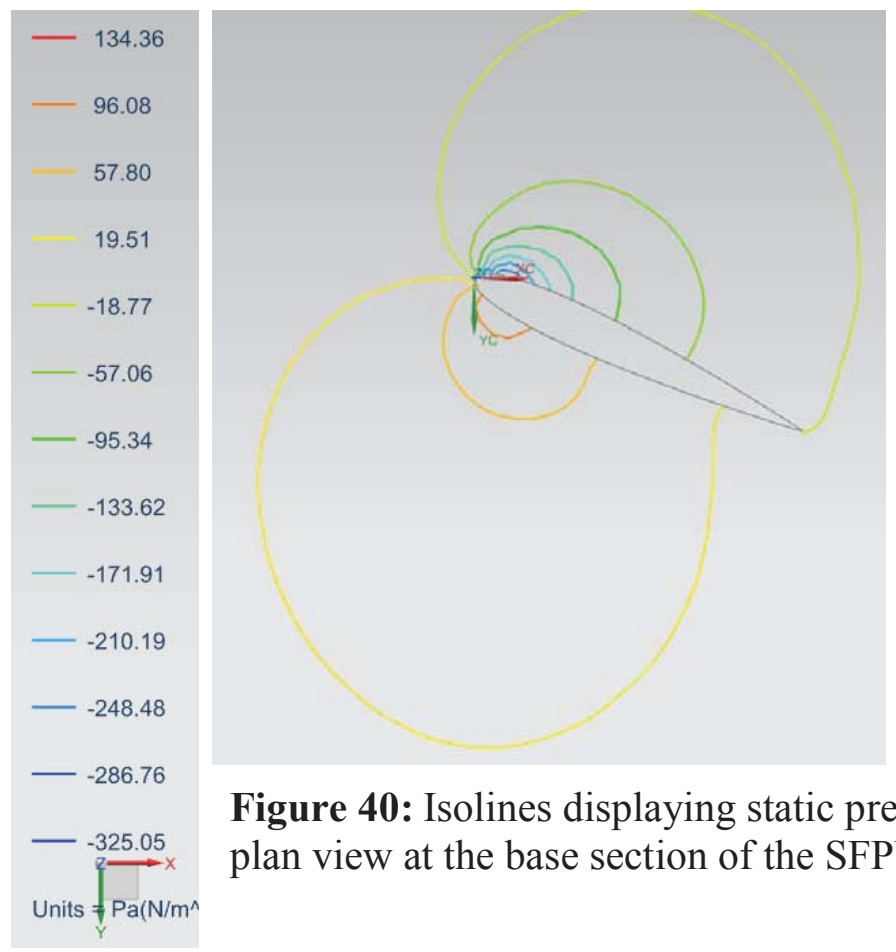

Figure 40: Isolines displaying static pressure distribution $\left(\mathrm{N} / \mathrm{m}^{2}\right)$ of the plan view at the base section of the SFPW at $\alpha=25^{\circ}, \mathrm{U}_{\infty}=0.5 \mathrm{mps}$

Figure 41 provides images of the mid-section of the SFPW and a three-dimensional isometric view for $25^{\circ}$ angle of attack. The mid-section flow regime in Figure 41(A) showed similar trends to the base-section. A decrease in velocity magnitude, colored in light blue, was shown at the leading edge of the suction side. The flow velocity reduced to a range from 0.21 to $0.31 \mathrm{mps}$. The direction of the flow also changed in this area. The flow split in this region to either point in the upward position toward the compression side, or the water flowed in the downward position to follow the length of suction surface. The water that moved over the top surface was shown to increase in velocity. Much of the top surface projected a dark blue coloration to show the decrease in velocity to as low as $0.05 \mathrm{mps}$. The direction of the flow followed the path around the surface. 

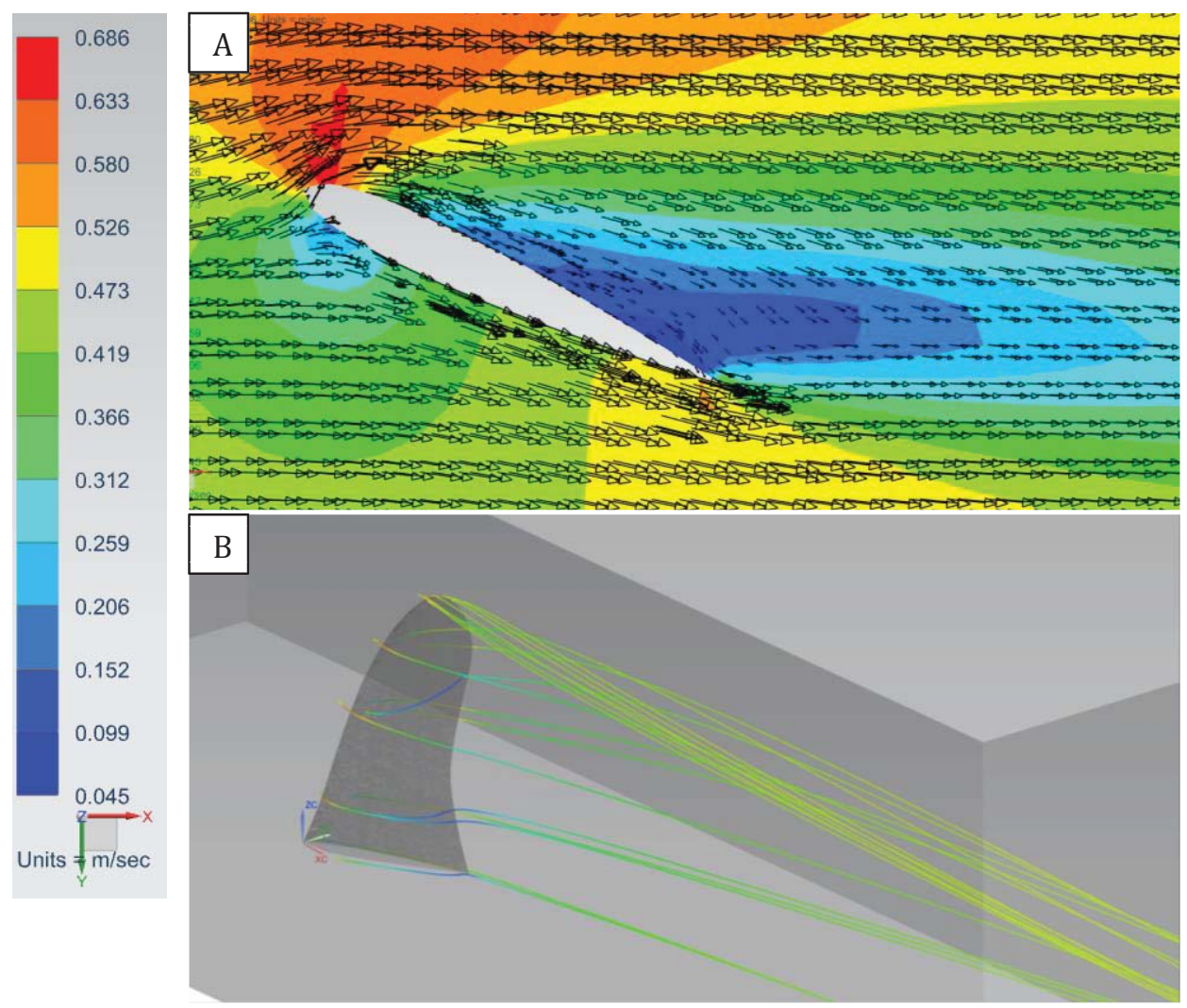

Figure 41: SFPW at $\alpha=25^{\circ}, \mathrm{U}_{\infty}=0.5 \mathrm{mps}$ (A) Velocity magnitudes and flow vectors, plan view at the mid-section (B) Streamlines, isometric view from trailing edge of the fin

The three-dimensional streamlines in Figure 41(B) can be compared to the flow vectors in Figure 42 through Figure 44. The flow vectors in these three figures are represented in a two-dimensional plane (ZC-XC). At the leading edge, the water flows horizontally, with little to no change in direction vertically; as depicted in Figure 42(A). As shown in Figure 42(B), there is an increase in velocity throughout the depth of the fin.

Figure 43(A) shows a change in the direction of the flow as the water intersects the fin. Approaching the suction side, the fluid in the upper region changed angle, moving towards the tip of the fin. On the same side, the flow at the base showed very little movement in the vertical direction. Comparing this to the compression side, downstream of the fin, the flow transitioned towards a more vertical direction. This alteration in the fluid's course of movement was more evident in the upper region of the fin. The 
streamlines in Figure 41(B) compliment the change in flow direction. In the upper region, downstream of the fin, fluid was shown to flow vertically towards the fin tip. This was within the zone of separation. Towards the base of the fin, the streamlines followed the path around the fin's surface. The tip of the fin showed evidence of shedding tip vortices as the fluid produced a twisting motion moving downstream. The small change in fluid direction at the tip of the fin can be seen in Figure 44(A). The flow path moved in a more vertical direction at both the tip and in the upper regions of the fin's trailing edge. The velocity decreased at the trailing edge to as low as $0.05 \mathrm{mps}$, as shown in Figure 44(B).

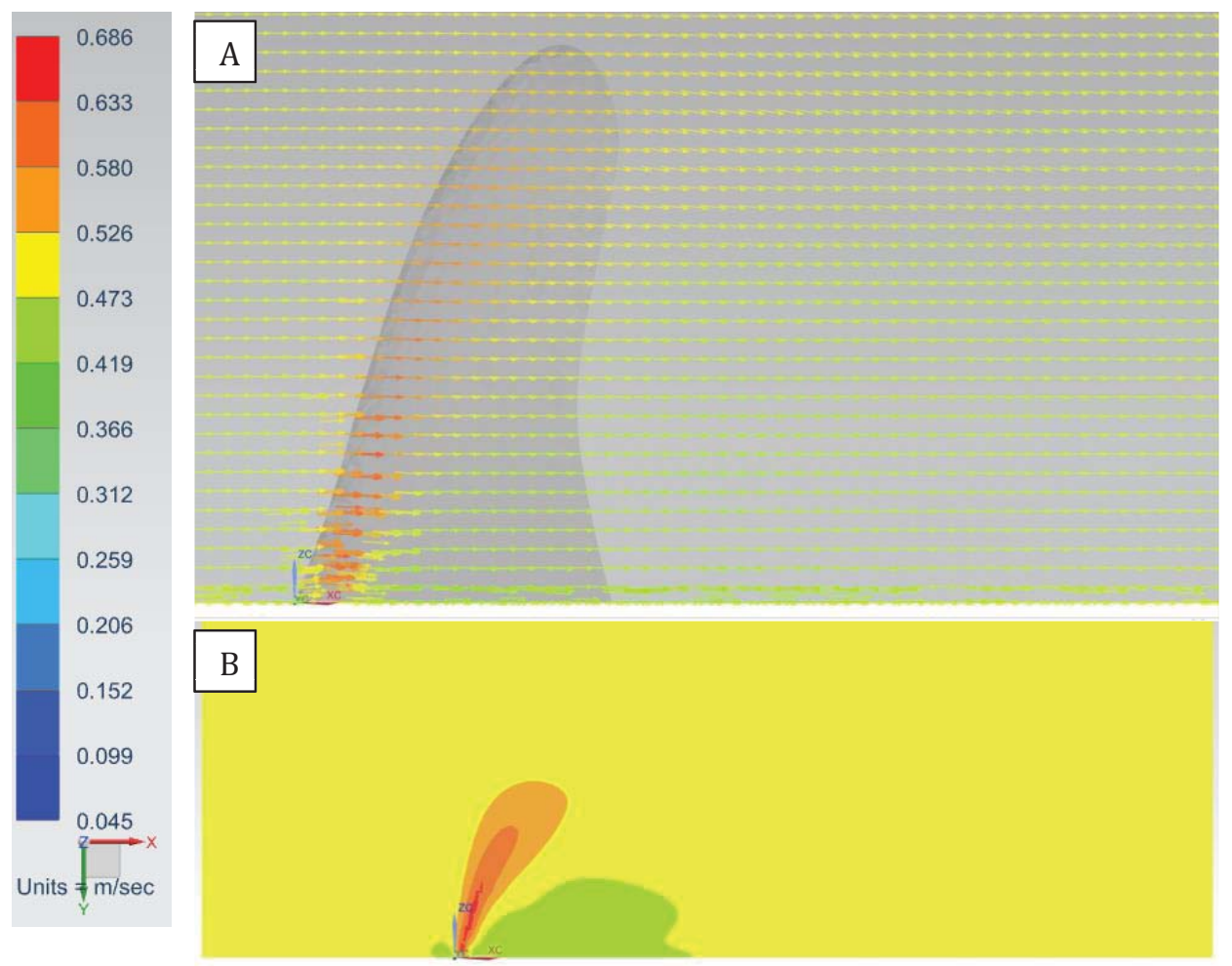

Figure 42: Profile view at the leading-edge section of the SFPW at $\alpha=25^{\circ}$, $\mathrm{U}_{\infty}=0.5 \mathrm{mps}$ (A) Flow vectors $(B)$ Velocity magnitudes 

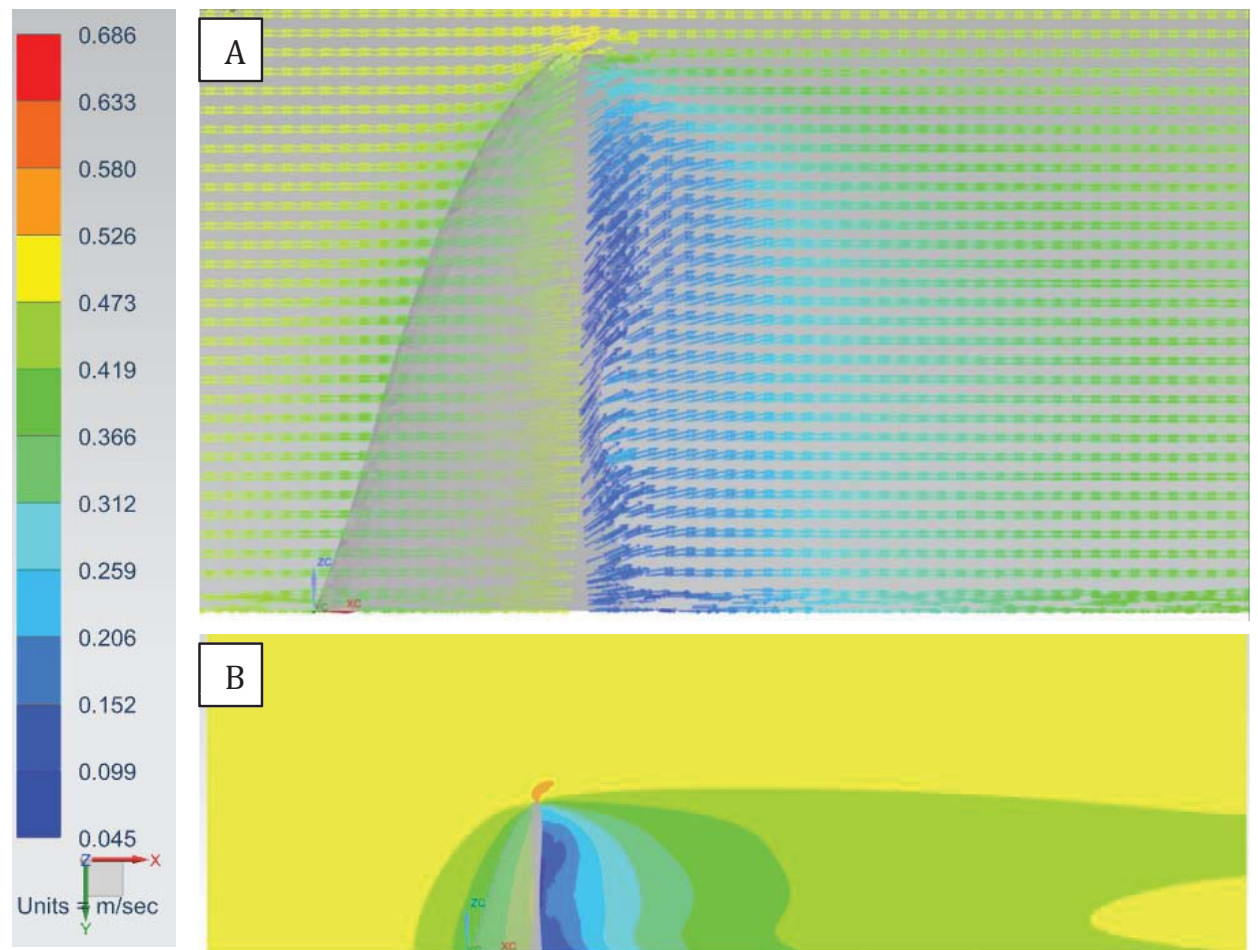

Figure 43: Profile view at the mid-edge section of the SFPW at $\alpha=25^{\circ}, \mathrm{U}_{\infty}=0.5 \mathrm{mps}$ (A) Flow vectors (B) Velocity magnitudes
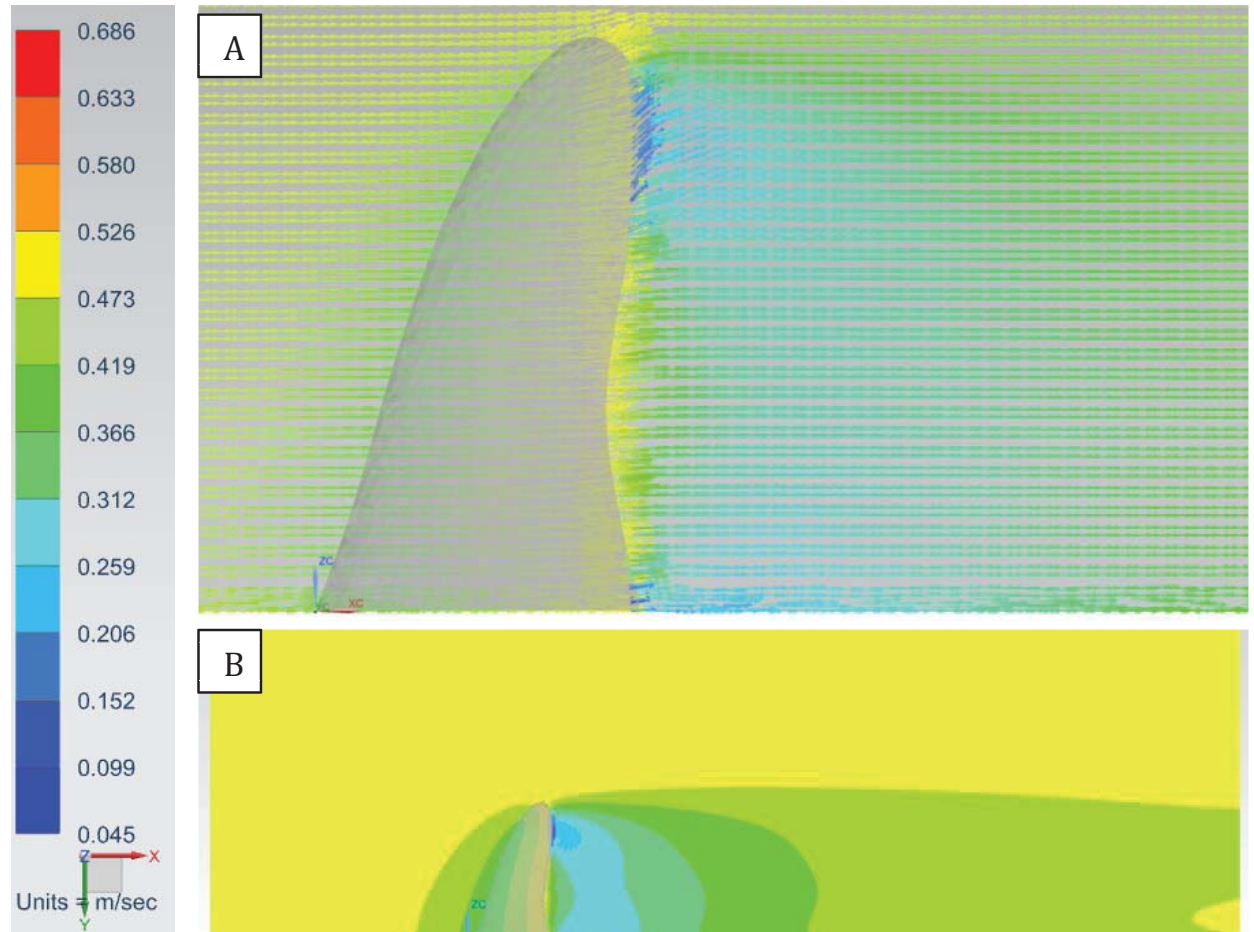

Figure 44: Profile view at the trailing-edge section of the SFPW at $\alpha=25^{\circ}$, $\mathrm{U}_{\infty}=0.5 \mathrm{mps}$ (A) Flow vectors $(B)$ Velocity magnitudes 
The maximum angle of attack for this project is presented in Figure 45 through Figure 50. Velocity magnitudes, flow vectors, and streamlines are represented within these figures in order to compare different views at $45^{\circ}$, as well as between different angles of attack. In Figure 45(A), there was a clear increase in velocity at the tip of the leading edge. There was a decrease in velocity on the suction side near the leading edge. The location of this stagnation region moved farther from the tip when compared to the SFPW at $25^{\circ}$. For the entire extent of the upper surface, there was a significant decrease in velocity.

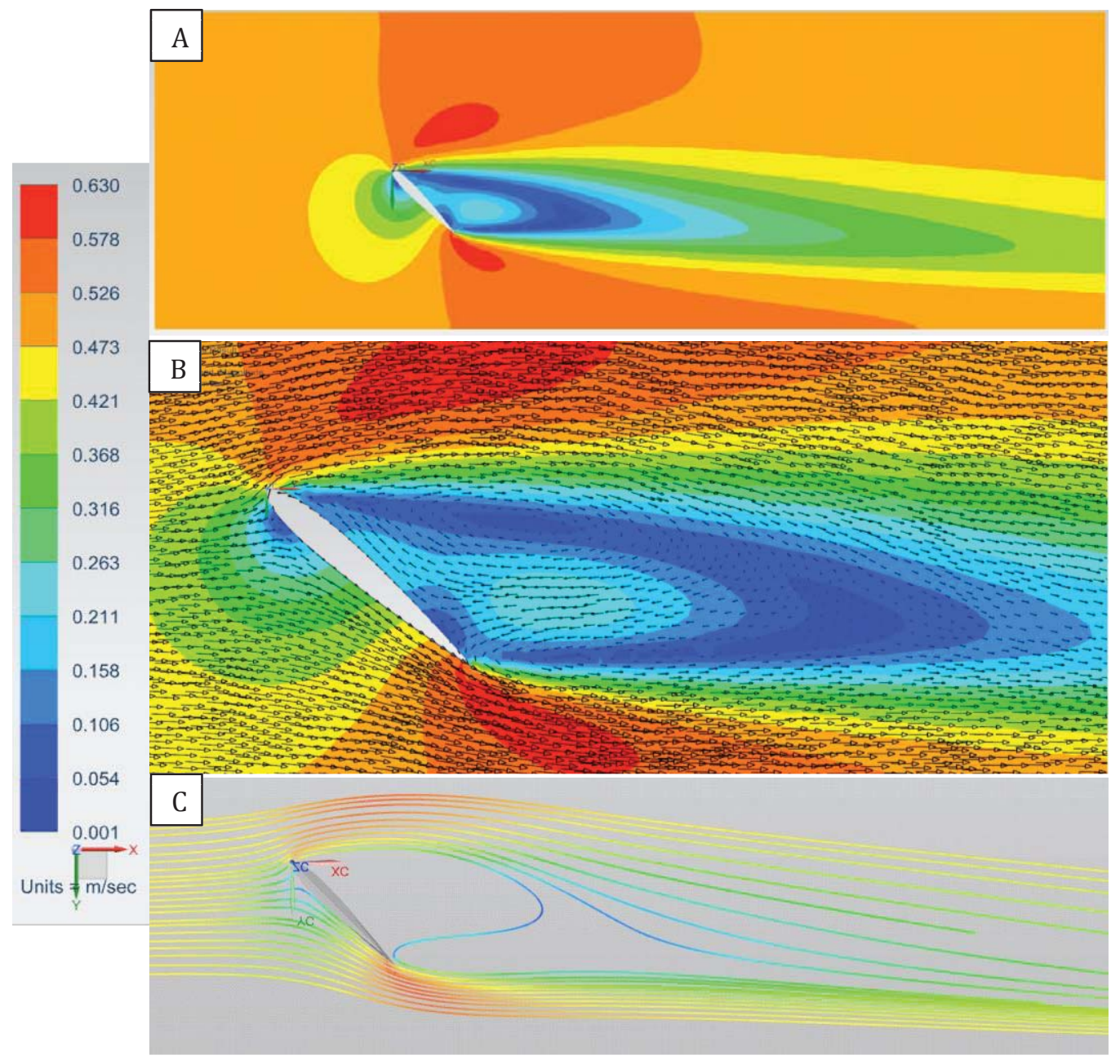

Figure 45: Plan view at the base section of the SFPW at $\alpha=45^{\circ}, \mathrm{U}_{\infty}=0.5 \mathrm{mps}$ (A) Velocity magnitudes. (B) Velocity magnitudes and flow vectors. (C) Streamlines 

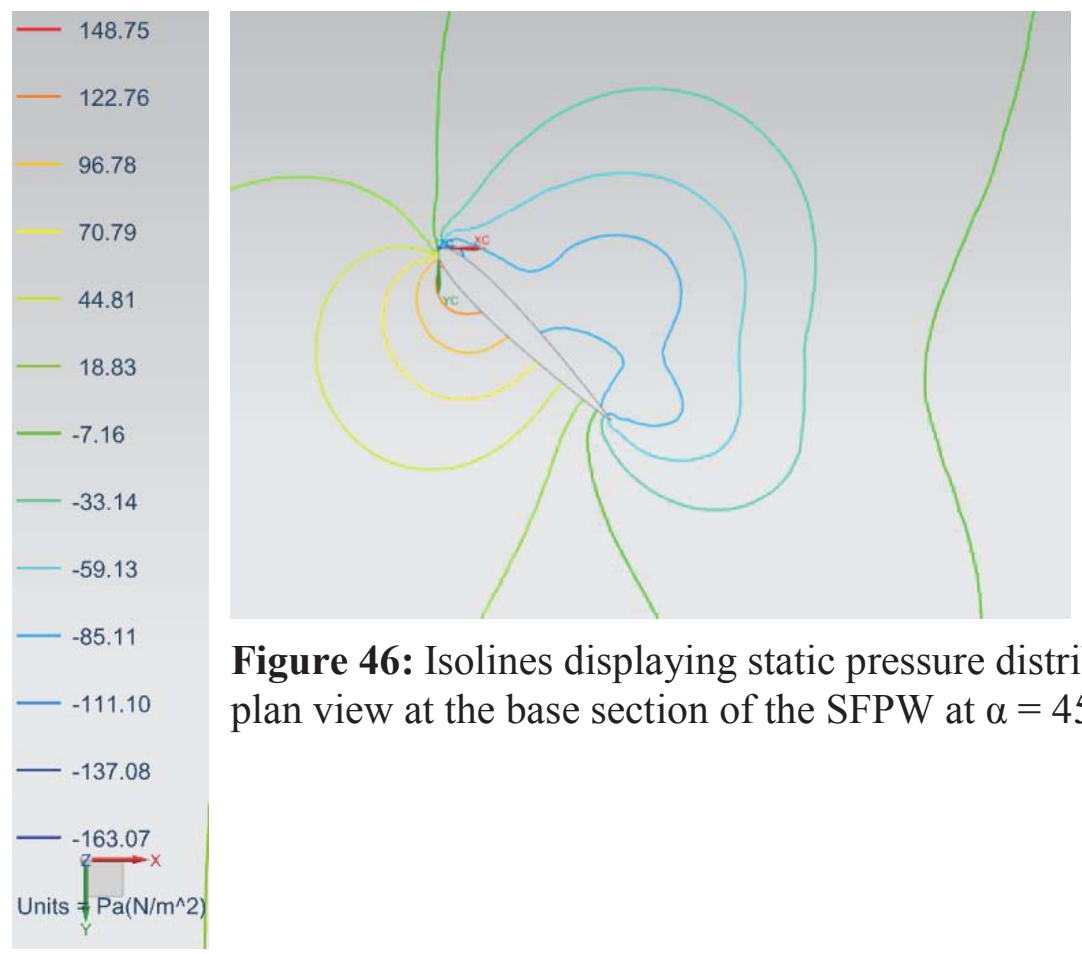

Figure 46: Isolines displaying static pressure distribution $\left(\mathrm{N} / \mathrm{m}^{2}\right)$ of the plan view at the base section of the SFPW at $\alpha=45^{\circ}, \mathrm{U}_{\infty}=0.5 \mathrm{mps}$

The flow vectors in Figure 45(B) and the streamlines in Figure 45(C) provide information about the flow field. At the leading edge on the suction side, velocity decreased to almost $0 \mathrm{mps}$ in the areas of dark blue. The static pressure increased in this area, as shown in Figure 46. In areas of dark red, an increase in velocity was shown at the leading edge of the compression side. An increase in velocity was also found at the tip of the trailing edge of the fin. Decreases in pressure to negative pressure values were found in these areas in Figure 46. On the compression side, velocity decreased immediately following the leading edge velocity increase. Eddies were identified within the fin's wake. From the leading edge, swirling fluid in a clockwise rotation was identified. A smaller counterclockwise rotation shed from the trailing edge towards the upper surface of the fin. Both of these fluid patterns were created behind the fin. The extent of this separation zone was greater in size than compared to the $25^{\circ}$ angle of attack. This area of low velocity was also evident in Figure 45(C) as there were few streamlines in the area downstream of the fin. 
Figure 47 provides the flow vectors at the mid-section of the fin and a three-dimensional isometric view of the streamlines. Similar flow field features, as compared to the basesection, were found at the mid-section. At the leading edge, a decrease in velocity was found near the suction side and an increase in velocity was found at the tip of the hydrofoil. Velocity also increased from the tip of the trailing edge and was found to be more substantial than the leading edge velocity increase. For the entire extent on compression side, velocity decreases dramatically. Swirling eddies occur in this area, shedding clockwise from the leading edge and counter-clockwise from the trailing edge. The eddies move in the vertical direction as swirling continues, which is shown in Figure 47(B). Tip vortices are also evident at the tip of the SFPW fin.

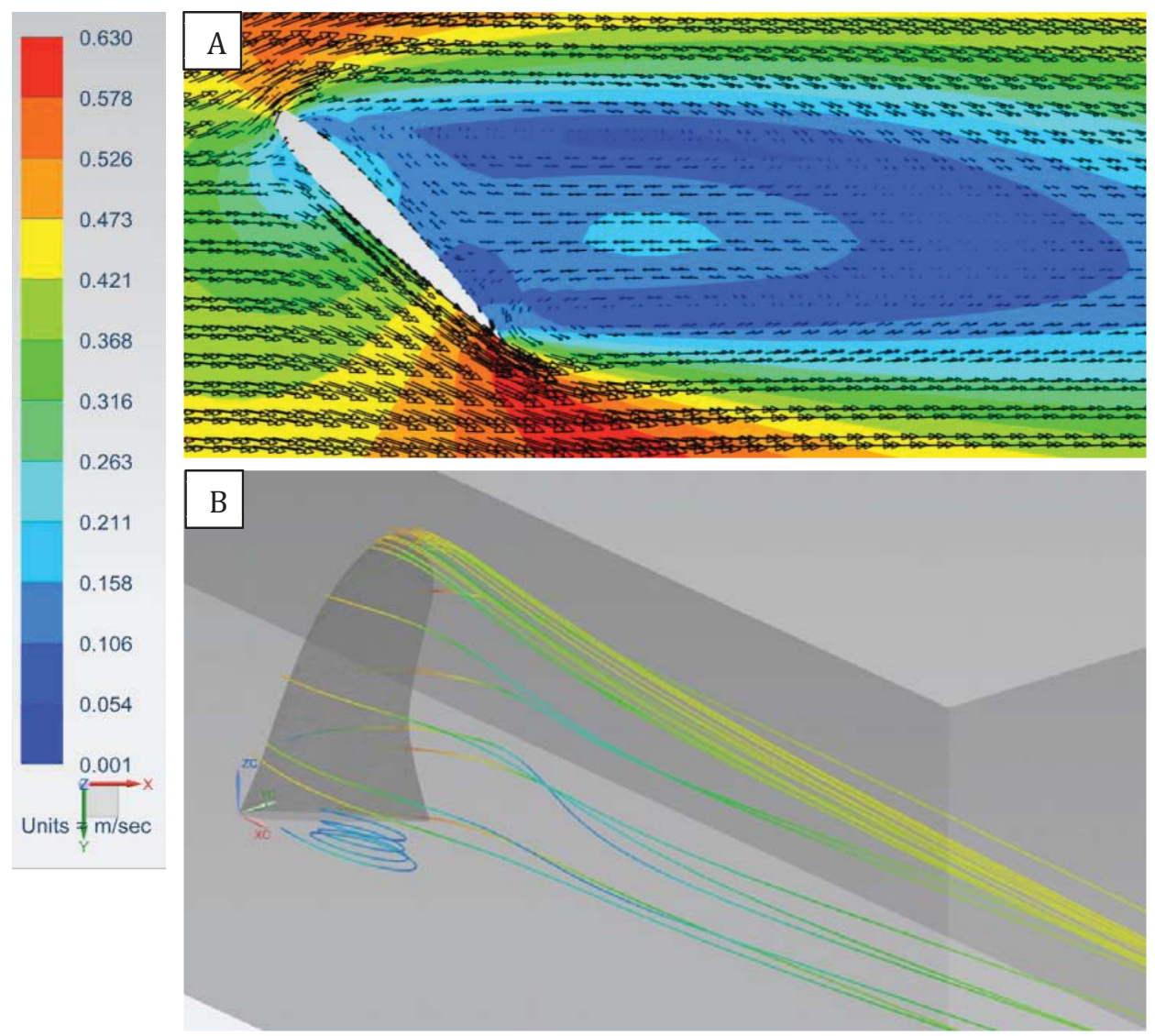

Figure 47: SFPW at $\alpha=45^{\circ}, \mathrm{U}_{\infty}=0.5 \mathrm{mps}(A)$ Velocity magnitudes and flow vectors, plan view at the mid-section (B) Streamlines, isometric view from trailing edge of the fin 
Figure 48 shows the flow vectors and velocity magnitudes, in the profile view of the SPFW, at the leading edge section. The velocity increased to about $0.63 \mathrm{mps}$ for the entire depth of the fin at the leading edge; Figure 48(B).

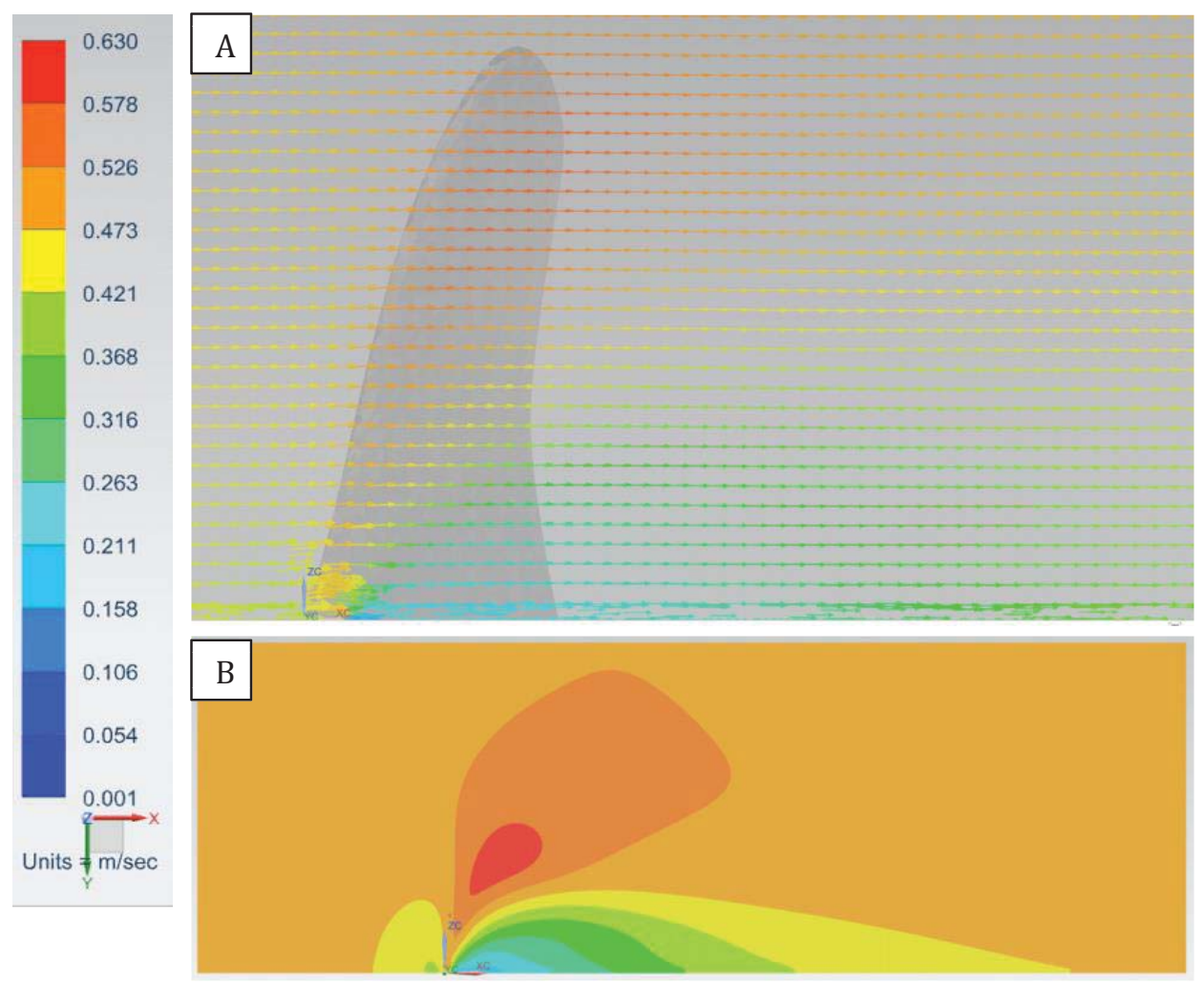

Figure 48: Profile view at the leading-edge section of the SFPW at $\alpha=45^{\circ}$, $\mathrm{U}_{\infty}=0.5 \mathrm{mps}(A)$ Flow vectors (B) Velocity magnitudes 
At the mid-edge section of the fin, flow swirled in the reverse direction of the fluid current. From the base-section to the mid-section, flow generally moved in the clockwise direction; as shown in Figure 49(A). At the mid-section, fluid moved in the counterclockwise direction, but then changed direction to the clockwise motion from the midsection to the tip of the fin. The size of the eddies and the low velocity area were larger near the base-section compared to the tip-section. Velocities decreased to almost zero in these sections; Figure 49(B). Off of the leading edge, at the tip of the fin, velocity increased ( 0.47 to $0.53 \mathrm{mps})$ and flow moved towards the vertical direction.

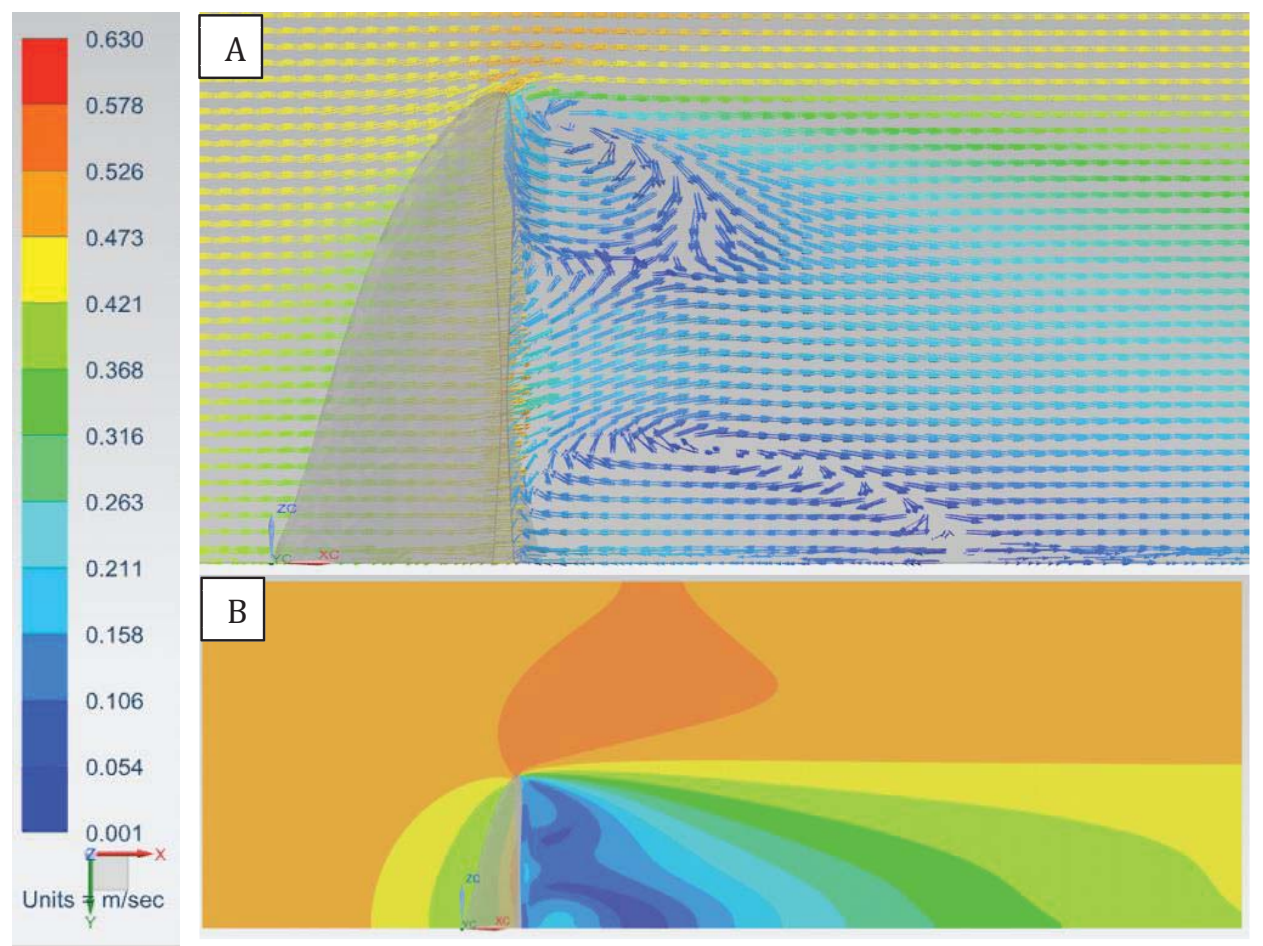

Figure 49: Profile view at the mid-edge section of the SFPW at $\alpha=45^{\circ}, \mathrm{U}_{\infty}=0.5 \mathrm{mps}$ (A) Flow vectors (B) Velocity magnitudes 
At the trailing edge of the fin, there was a significant increase in velocity, as shown in Figure 50(B). This was also accompanied by changes in flow direction. Near the tip of the fin, the fluid moved towards the upward direction; this is depicted in Figure 50(A).

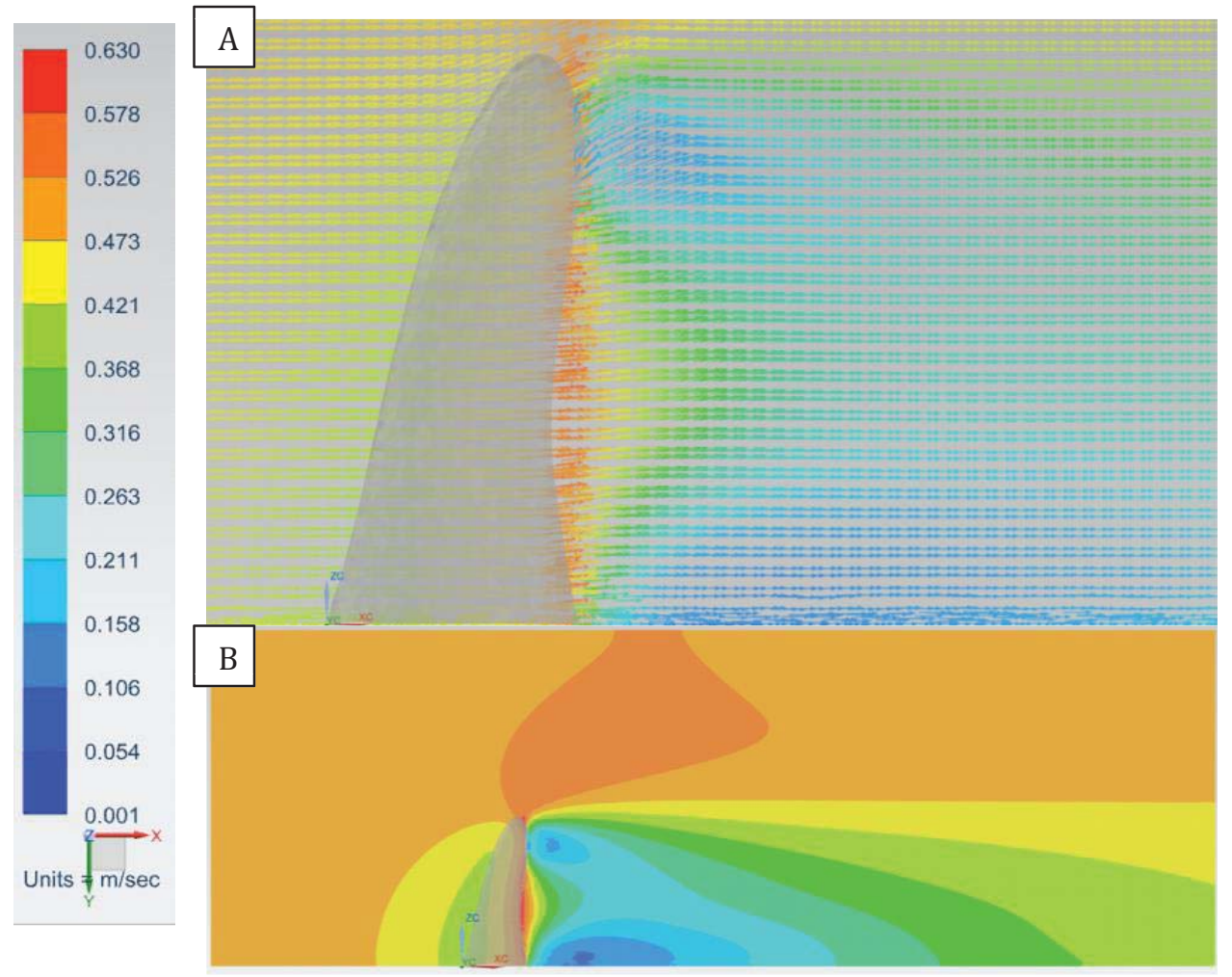

Figure 50: Profile view at the trailing-edge section of the SFPW at $\alpha=45^{\circ}$, $\mathrm{U}_{\infty}=0.5 \mathrm{mps}(A)$ Flow vectors $(B)$ Velocity magnitudes 


\subsubsection{LABORATORY VISUAL ANALYSIS}

To receive a complete understanding of the flow field around the SFPW, laboratory visual experiments were done. Screenshots, in one second intervals, of the slow motion video were taken to present in this report. In the plan view images, the white circle is a circular underwater viewer, which was used for clear images below the water surface. The images were analyzed, along with the CFD visual images, for contrasts and comparisons. Four angles of attack were chosen $\left(0^{\circ}, 10^{\circ}, 25^{\circ}, 45^{\circ}\right)$ to present in the body of this thesis.

At $0^{\circ}$ angle of attack, Figure 51 provides images for the progression of the dye injections, in one second intervals, along the base of the SFPW fin. From the first image, dye was shot upstream of the leading edge to allow the dye to move around the fin. In the following three images, the dye was shown to move evenly on both sides of the fin. The fin was symmetric, which allowed for even water flow on the upper and lower surfaces. In both the third and fourth image, the dye was thinner directly behind the trailing edge.

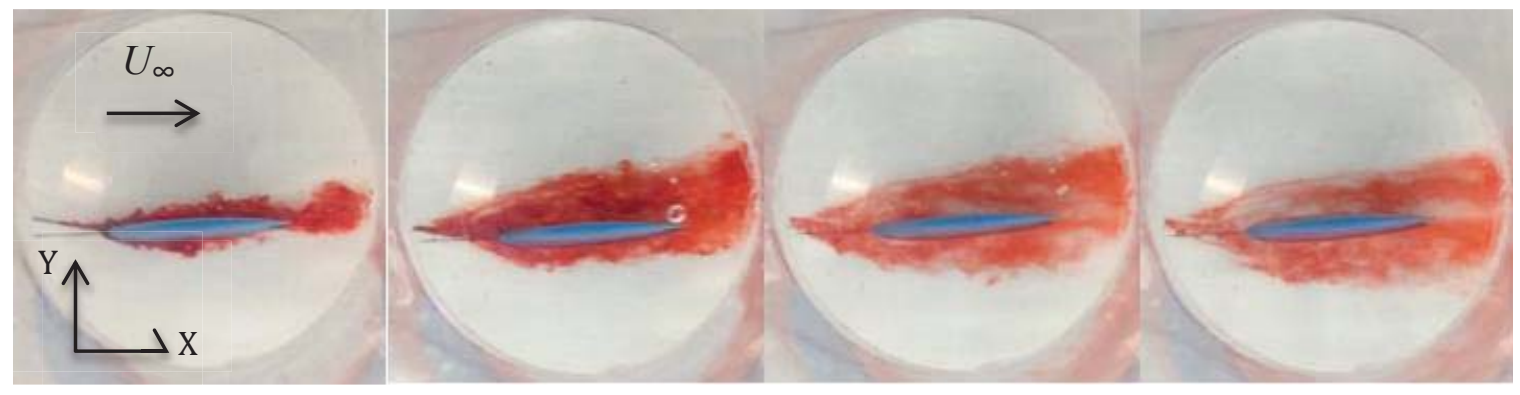

Figure 51: Plan view of the flow past the SFPW at $\alpha=0^{\circ}, \mathrm{U}=0.5 \mathrm{mps}$ using dye injections at the base section

At the mid-section, in Figure 52, a comparable trend was found. The dye moved over the surface in a similar fashion. Even water flow around both sides of the fin occurred. The dye leaving the nozzle of the dye-injector had a wavy, turbulent movement. This was found in all four mid-section images and in the video evidence. 


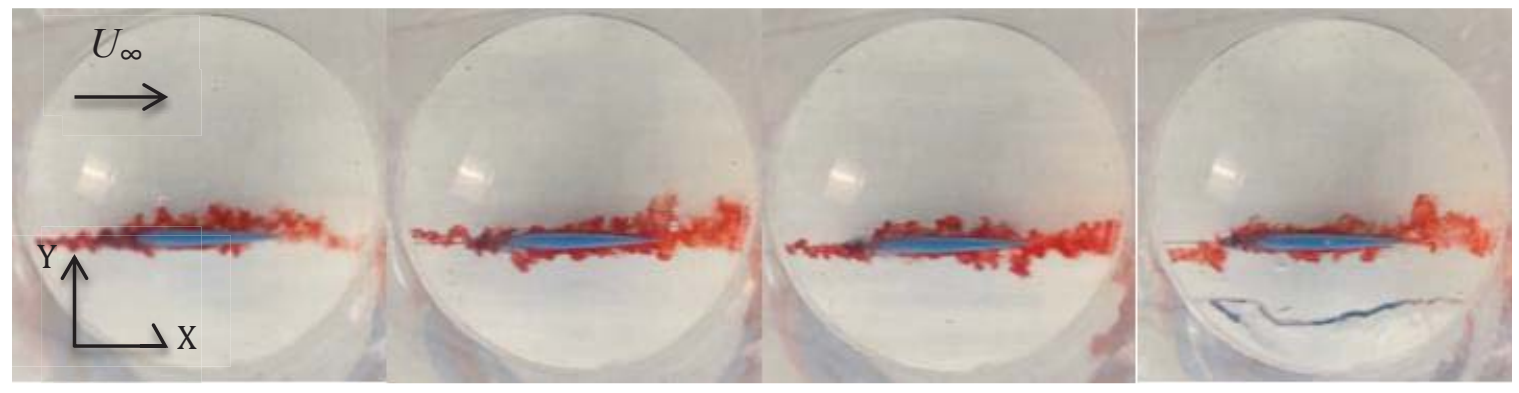

Figure 52: Plan view of the flow past the SFPW at $\alpha=0^{\circ}, U_{\infty}=0.5 \mathrm{mps}$ using dye injections at the mid-section

Figure 53 presents the SFPW, at $0^{\circ}$ angle of attack, in the profile position. Dye was injected near the base of the fin. The dye showed little effect from the fin as it moved downstream. Small trailing vortices occurred directly after encountering the fin, but were not clear in all images. The dye quickly dissipated once the injection process ceased.
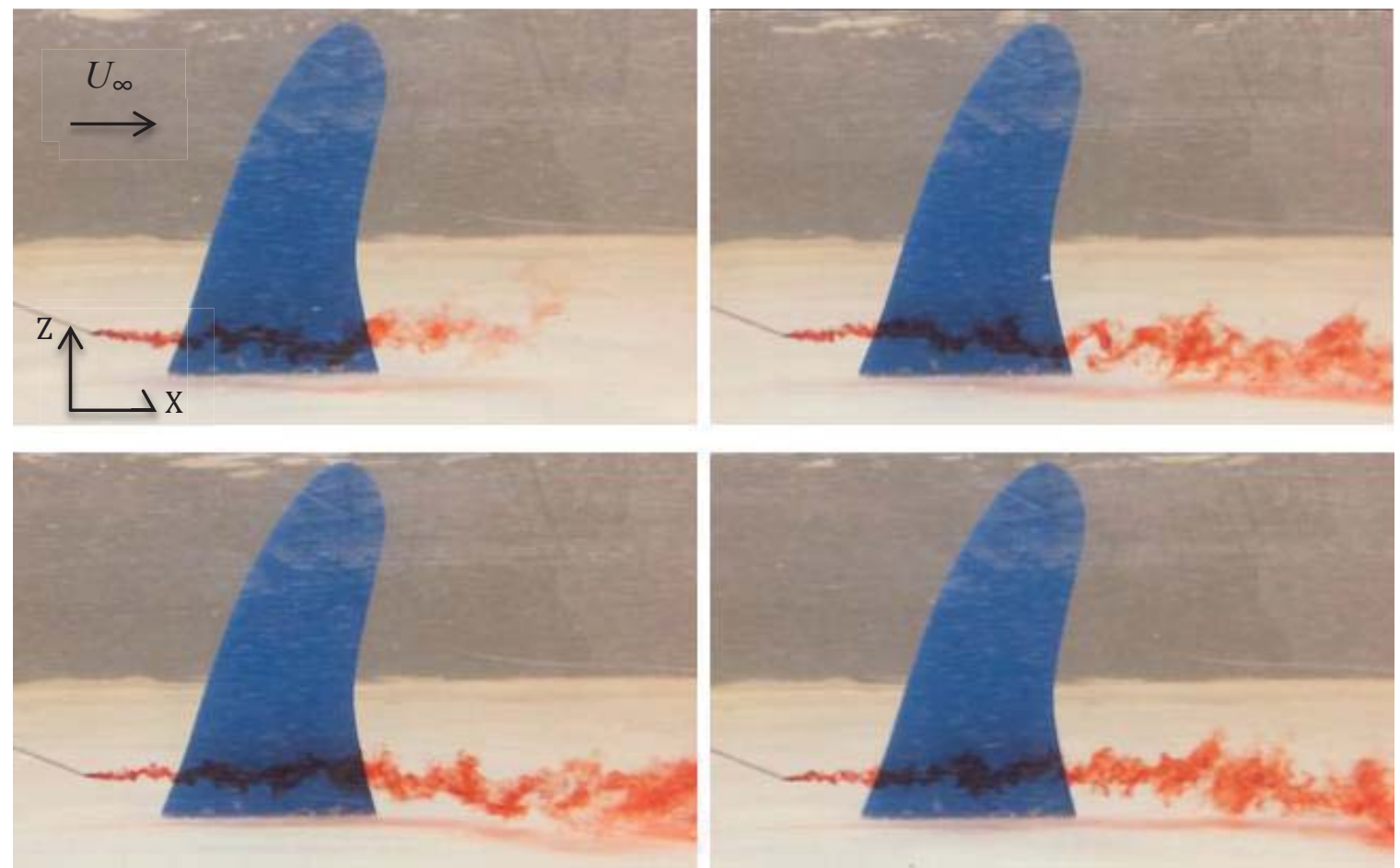

Figure 53: Profile view of the flow past the SFPW at $\alpha=0^{\circ}, U_{\infty}=0.5 \mathrm{mps}$ using dye injections at the base section 
Figure 54 provides dye injections at the mid-section of the profile view. It was discovered that these flow patterns resembled the base-section. As the dye passed over the fin, small disruptions in the horizontal flow were seen. Similar eddy patterns were found directly downstream of the fin. It was undetermined whether these were shedding vortices from the trailing edge of the fin, natural turbulence in the water channel, or the dye injection method. Overall, the dye showed that the water flowed in the horizontal direction, with only small movements in the vertical direction.
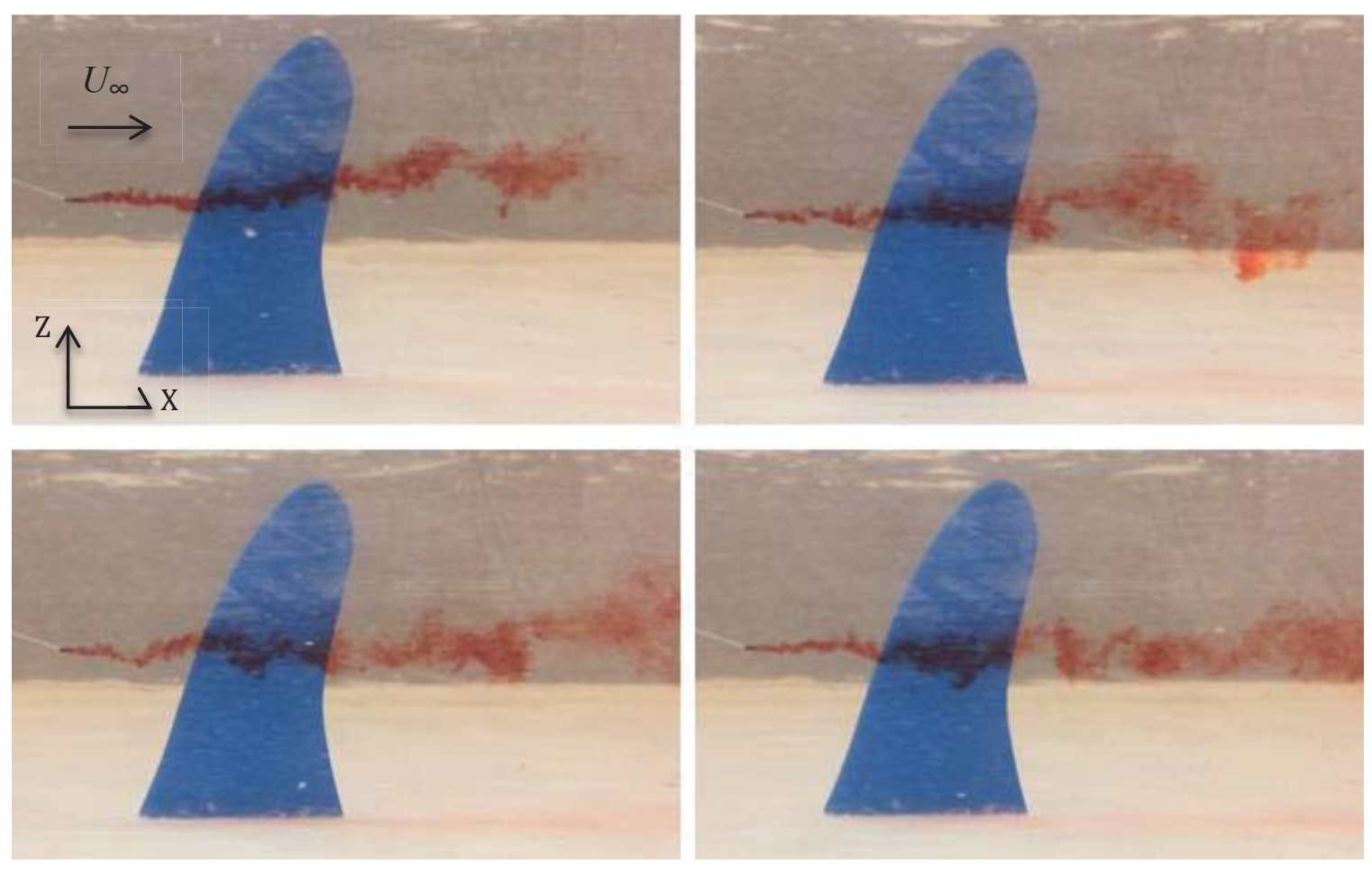

Figure 54: Profile view of the flow past the SFPW at $\alpha=0^{\circ}, U_{\infty}=0.5 \mathrm{mps}$ using dye injections at the mid-section

At the tip of the fin, the dye was added to determine if wing tip vortices were visible; Figure 55. As the dye approached the fin, it moved in a dense straight line. Passing over the fin tip and in the wake of the fin, the dye dissolved as it spread out slightly. It was unclear whether the small movements in the wake of the fin were due to turbulence in the water channel or from the trailing tip vortices. 


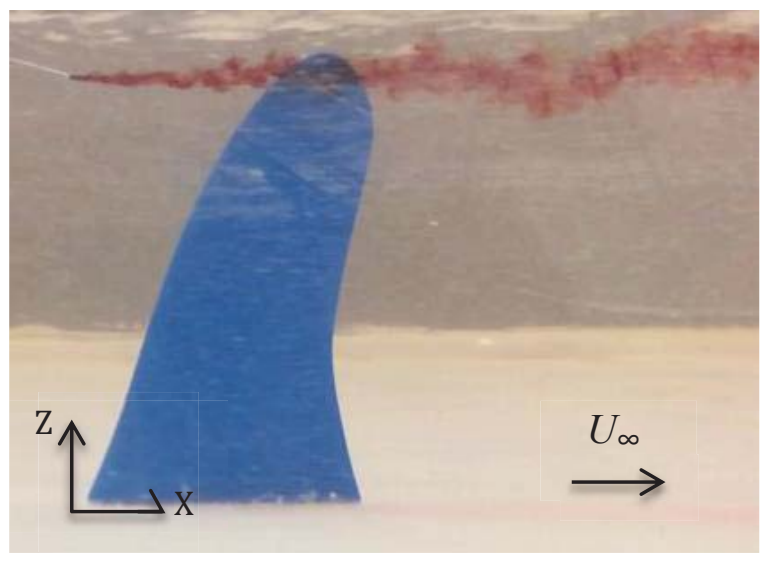

Figure 55: Profile view of the flow past the SFPW at $\alpha=0^{\circ}, U_{\infty}=0.5 \mathrm{mps}$ using dye injections at the fin tip
For $10^{\circ}$ angle of attack, the flow continued to flow smoothly over the hydrofoil. The flow stayed attached without signs of flow separation. In Figure 56(A), the dye moved from the leading edge over the compression side of the fin. From the video, it can be seen that there was a slight increase in velocity near the leading edge on this side. The dye flowed in a thinner more condensed line around this compressed edge, but dispersed more as it moved towards the trailing edge. Near the trailing edge, the flow velocity slowed down slightly. From the leading to trailing edge on the compression side, the flow did not separate from the fin's surface.

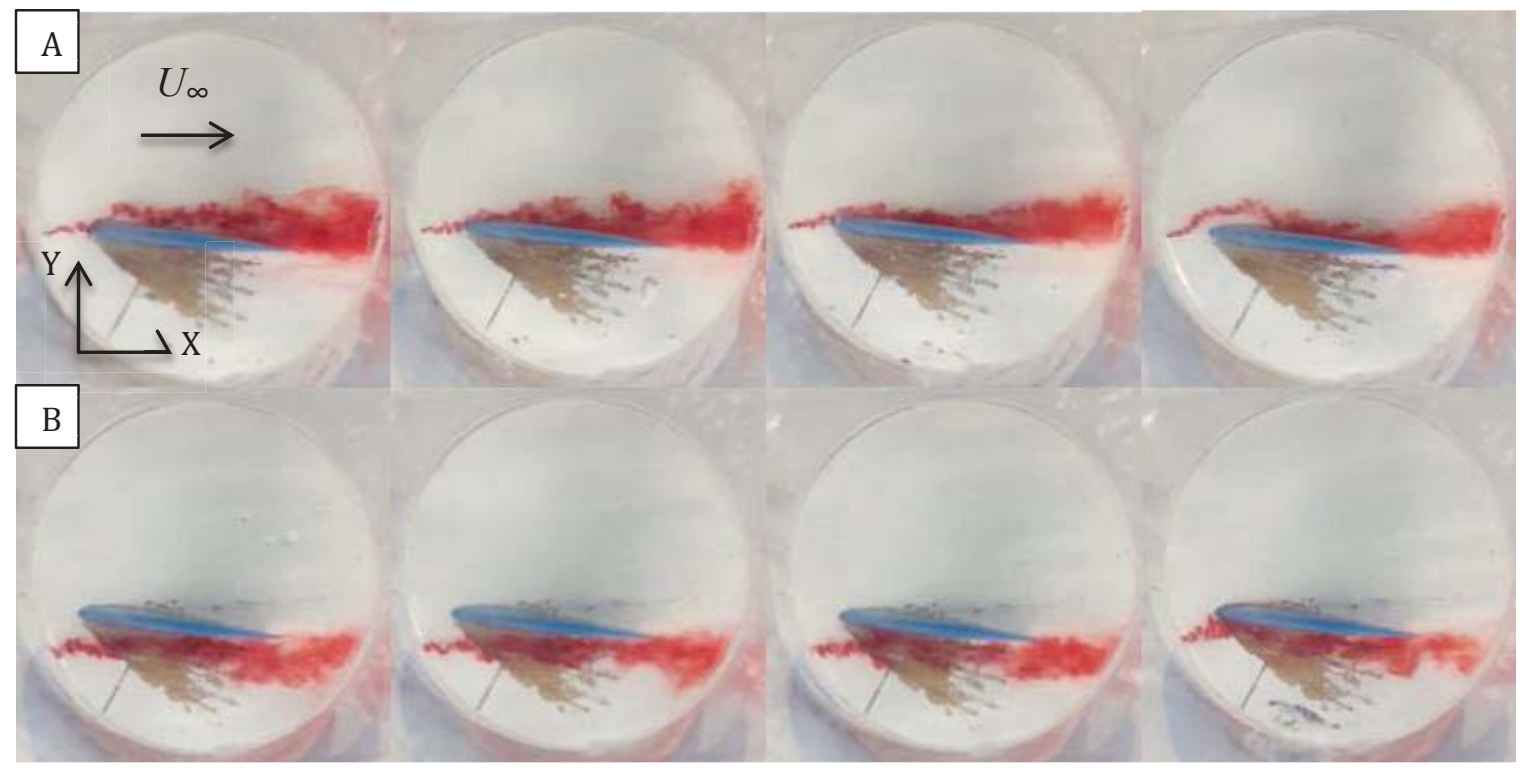

Figure 56: Plan view of the flow past the SFPW at $\alpha=10^{\circ}, U_{\infty}=0.5 \mathrm{mps}$ using dye injections at the base section (A) compression side. Sequence from left to right (B) suction side. Sequence from left to right

In Figure 56(B), the dye was injected on the suction side at the base of the SFPW. From the video, it was shown that the velocity was slower at the leading edge compared to the trailing edge. When dye was placed at the leading edge, the dye stayed attached to the fin 
for the entire chord-length. It was difficult to determine the thickness of the dye at the leading and trailing edges, from the hydrofoil.

Dye injections at the fin's base section are presented in the profile view, at $10^{\circ}$ angle of attack, in Figure 57 and Figure 58. The dye was added at the leading edge of the compression and suction side. Each side showed the fluid moved in direction of flow, with little movement in the vertical Z-direction. In Figure 57, the dye moved past the leading edge in a thin condensed line. As it moved past the trailing edge, the flow separated and small trailing vortices were vaguely noticeable. These flow patterns supported the trends found in the plan view.
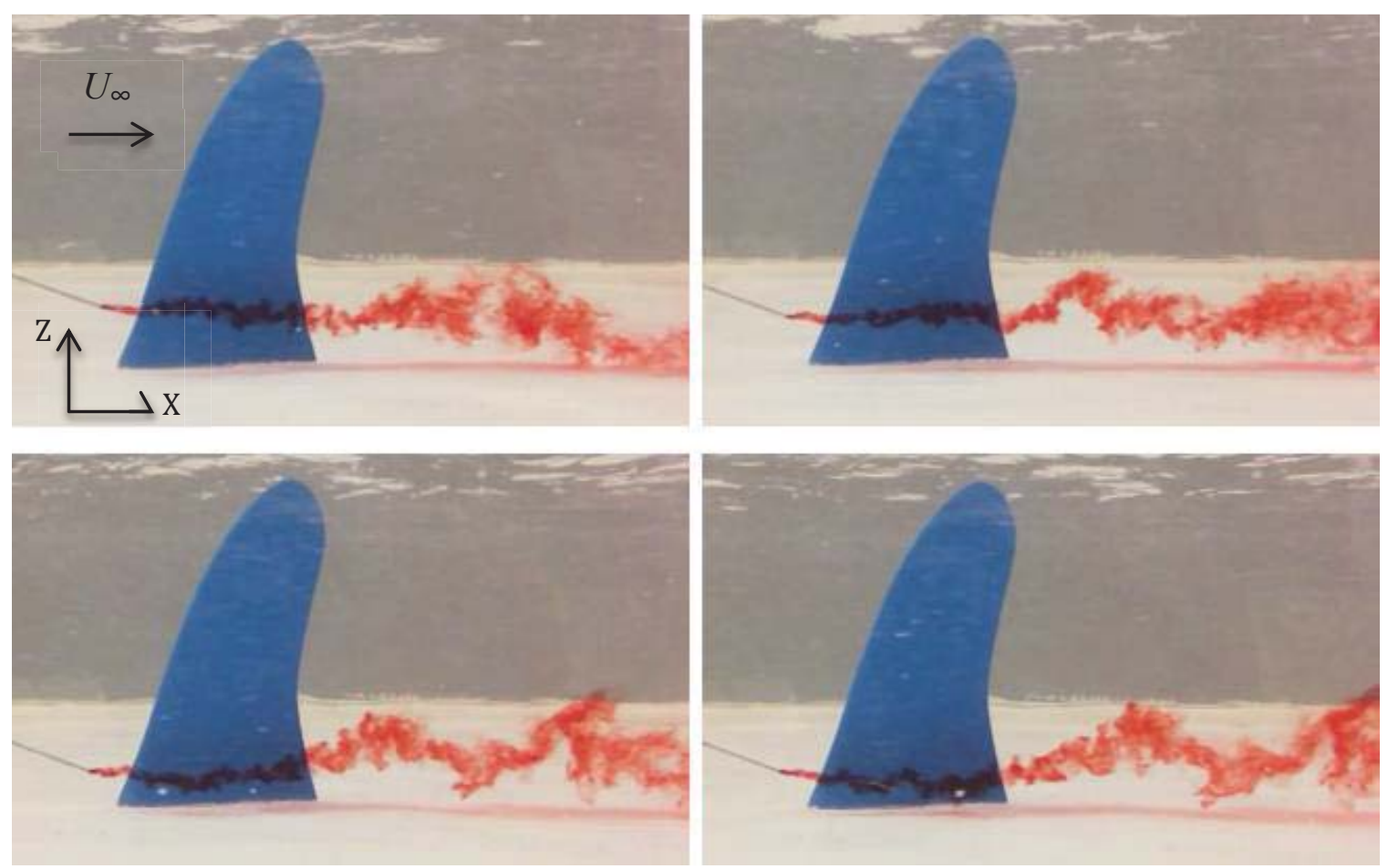

Figure 57: Profile view of the flow past the SFPW at $\alpha=10^{\circ}, U_{\infty}=0.5 \mathrm{mps}$ using dye injections on the compression side at the base section. Sequence from left to right

The suction side, in Figure 58, shows the dye moving horizontally in the direction of the flow. The patterns of the dye illustrated the chaotic behaviors of turbulent flow in the water. There was one hiccup that is shown in all four sequential images, which revealed a bump in the dye line. It started in the first image directly behind the trailing edge, and then moved increasingly downstream in the following images. This was not shown to be a common 
trend in the flow patterns, but more of an independent influence. In the first image, some dye shed onto the compression side. Turbulent flow patterns were also shown on the suction side.
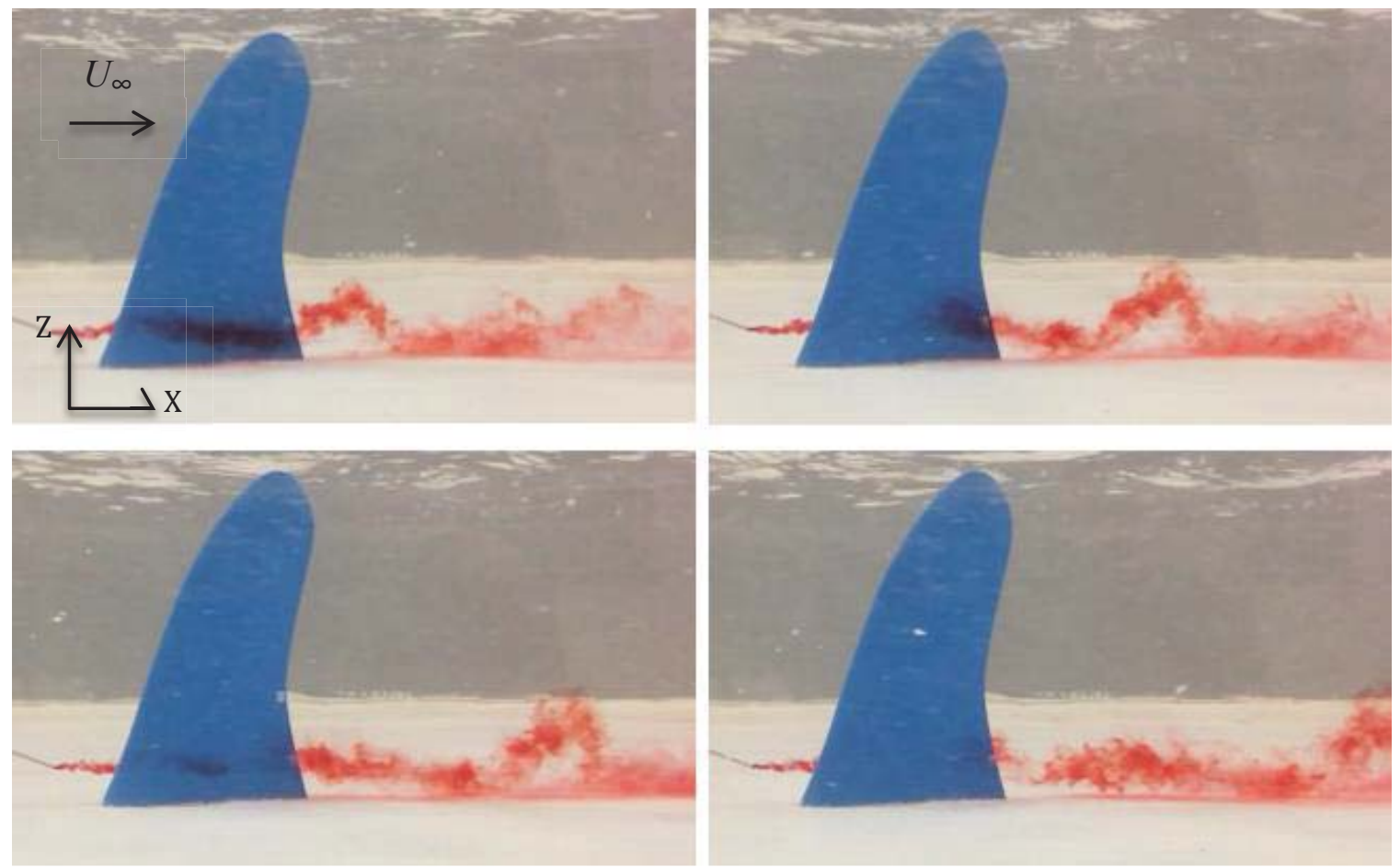

Figure 58: Profile view of the flow past the SFPW at $\alpha=10^{\circ}, U_{\infty}=0.5 \mathrm{mps}$ using dye injections on the suction side at the base section. Sequence from left to right

The mid-section plan view images, at $10^{\circ}$ angles of attack, are shown in Figure 59. Flow separation was not apparent on either side of the hydrofoil. As shown in the video, velocity differences were still obvious on the compression side; Figure 59(A). Velocity increased at the leading and then decreased moving towards the trailing edge. The dye stayed attached to the fin as it moved over the surface. On the suction side, it visually appeared that the dye moved slower near the leading edge and increased in velocity as it moved towards the trailing edge. This was determined from the video evidence. Without physical measurements, the predictions cannot be proven or quantified. From Figure 59(B), the dye avoided the leading edge and dispersed more as it moved towards the tail of the hydrofoil. The fourth image on Figure 59(B) shows an attached flow from the tip to the tail. From the 
nozzle of the dye-injector, the dye moved in a wavy-turbulent manner. This same movement was shown in all the images for the mid-section plan view.

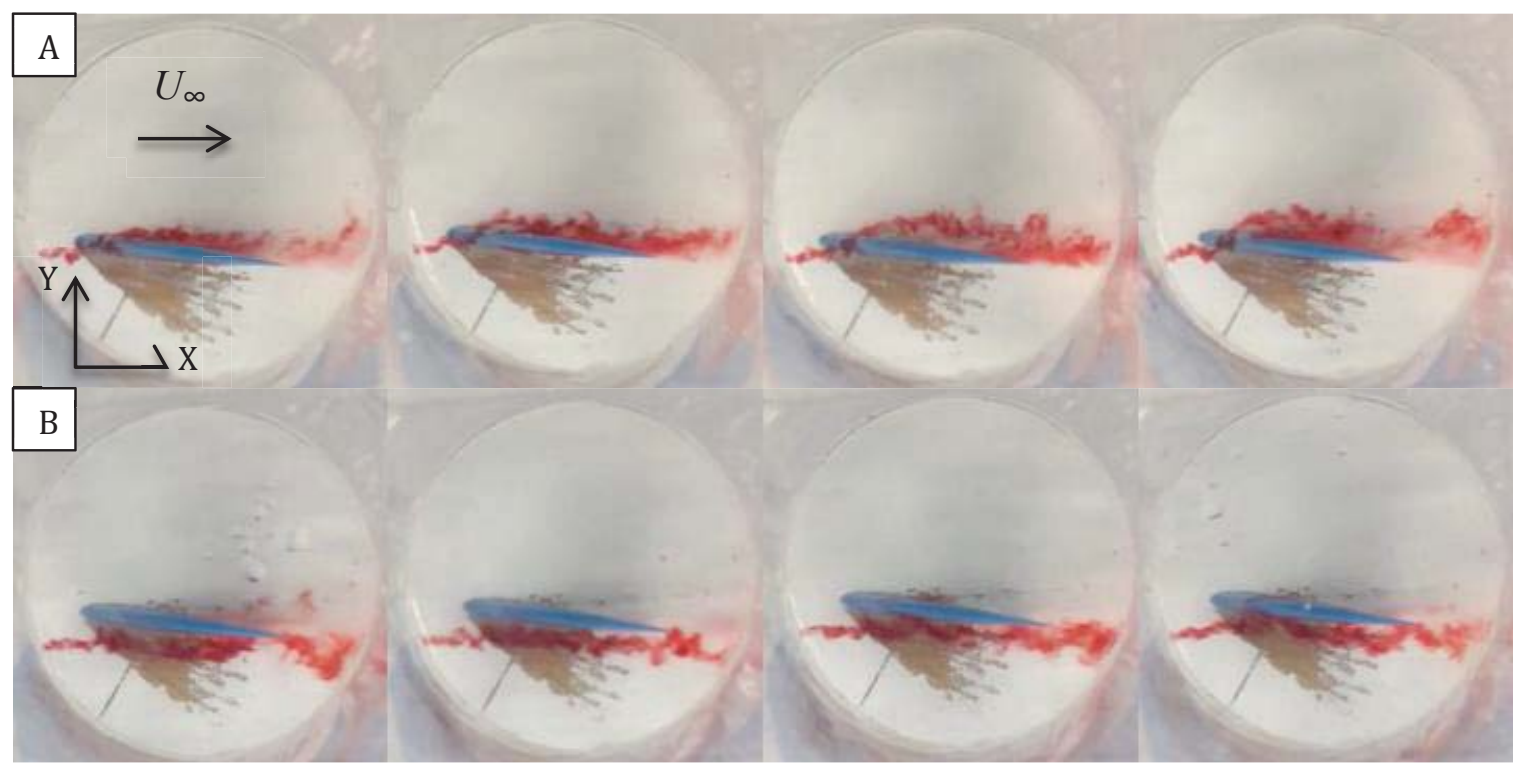

Figure 59: Plan view of the flow past the SFPW at $\alpha=10^{\circ}, U_{\infty}=0.5 \mathrm{mps}$ using dye injections at the mid-section (A) compression side. Sequence from left to right (B) suction side. Sequence from left to right.

At $10^{\circ}$ angles of attack, the mid-section profile view images are shown in Figure 60 and Figure 61. Figure 60 presents the dye added at the leading edge of the compression side, while Figure 61 illustrates the suction side. The profile images coincided with the plan view flow patterns. At the leading edge of the compression side, the dye was condense and flowed in a straight path. As the dye moved towards the trailing edge, it dispersed more. The pattern of the flow showed some turbulence might have been present. Following the fin, the dye spread in a turbulent manner that could be considered trailing vortices. In Figure 61, the dye dispersed in a similar way following the trailing edge. At the leading edge, some dye leaked to the compression side in the second and fourth image. It was difficult to determine the velocity differences at the leading and trailing edge of the fin from both the compression and suction videos. 

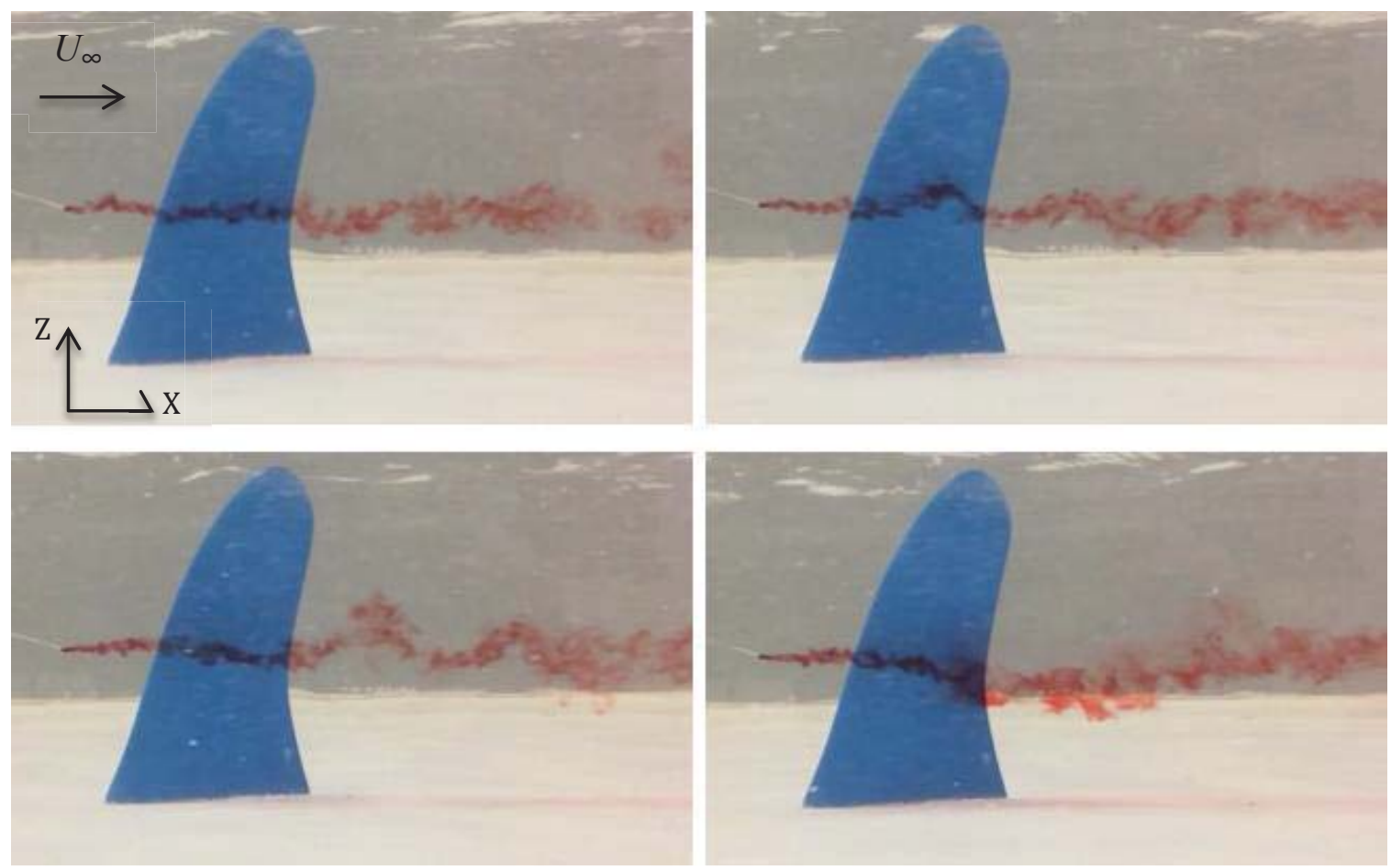

Figure 60: Profile view of the flow past the SFPW at $\alpha=10^{\circ}, U_{\infty}=0.5 \mathrm{mps}$ using dye injections on the compression side at the mid-section. Sequence from left to right.
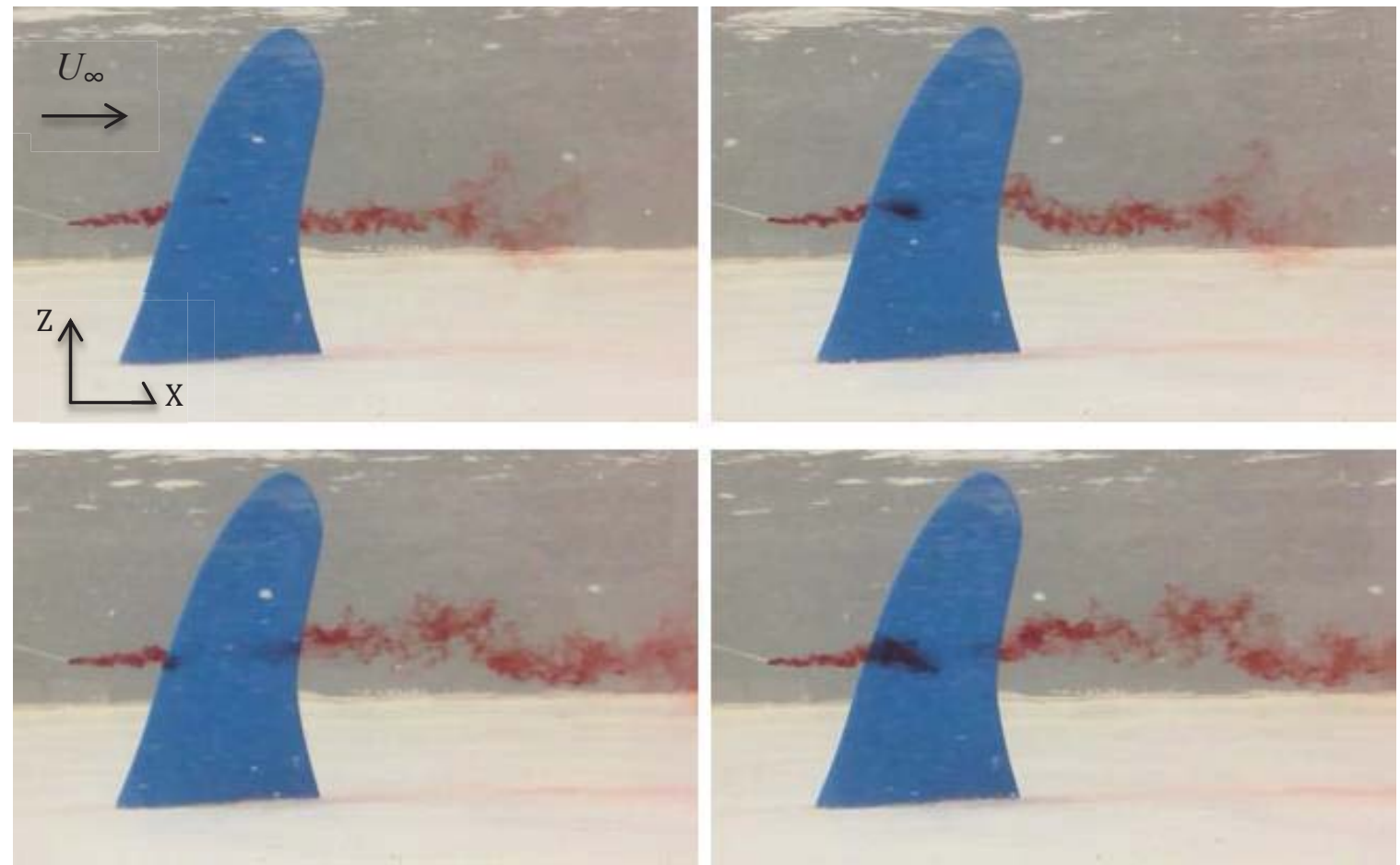

Figure 61: Profile view of the flow past the SFPW at $\alpha=10^{\circ}, U_{\infty}=0.5 \mathrm{mps}$ using dye injections on the suction side at the mid-section. Sequence from left to right. 
The flow around the fin tip was observed at $10^{\circ}$ angle of attack. As displayed in Figure 62 , the dye was injected into the water upstream of the leading edge. This caused the dye to move in the horizontal Xdirection until the dye encountered the fin. At that point, the dye flowed over the fin tip and then dramatically dipped below the dye insertion level. This differed from $0^{\circ}$, in

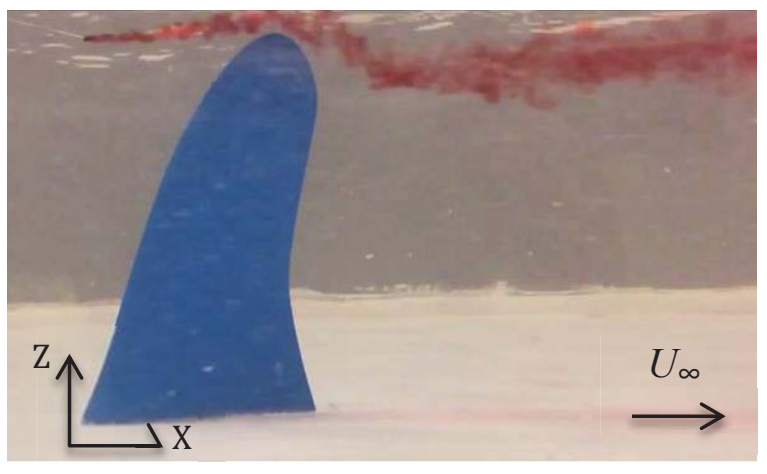

Figure 62: Profile view of the flow past the SFPW at $\alpha=10^{\circ}, U_{\infty}=0.5 \mathrm{mps}$ using dye injections at the fin tip which the dye moved past the fin without changing course in the vertical Z-direction. Tip vortices were observed.

Repositioning the SFPW fin to $25^{\circ}$, changed the flow field near the fin and in the downstream position. Figure 63 presents dye injections on the compression and suction side in sequential order. Referring to Figure 63(A), the first image shows that the dye does not stay attached to the fin's surface as it moves around the leading edge. The second image illustrates the dye wrapping in towards the tail of the fin, with some signs of turbulent vortices. In the zone between the leading and trailing edge, the dye moved in the opposing direction of the oncoming fluid velocity. As time progressed, the dye built up in this zone of low to zero velocity. The video evidence displayed the point at which the dye injections were withdrawn, but the dye continued to sit within this dead velocity zone. It was found that it took several seconds for the dye to completely dilute and move downstream from this area.

In Figure 63(B), the dye is inserted on the suction side. Due to the low velocity near the leading edge on this side, the dye avoided the leading edge area. Towards the tail of the fin, the dye made contact with the fin's surface. Moving away from the trailing edge, the dye curled towards the upper side of the fin. In the second and third images, the dye moved in the opposite direction of flow as eddies formed in the zone of low velocity. The dye was shown to build up in this area as trailing vortices continued to form. For both the 
compression and suction side, turbulence was shown to be present, as the dye did not show signs of laminar flow.

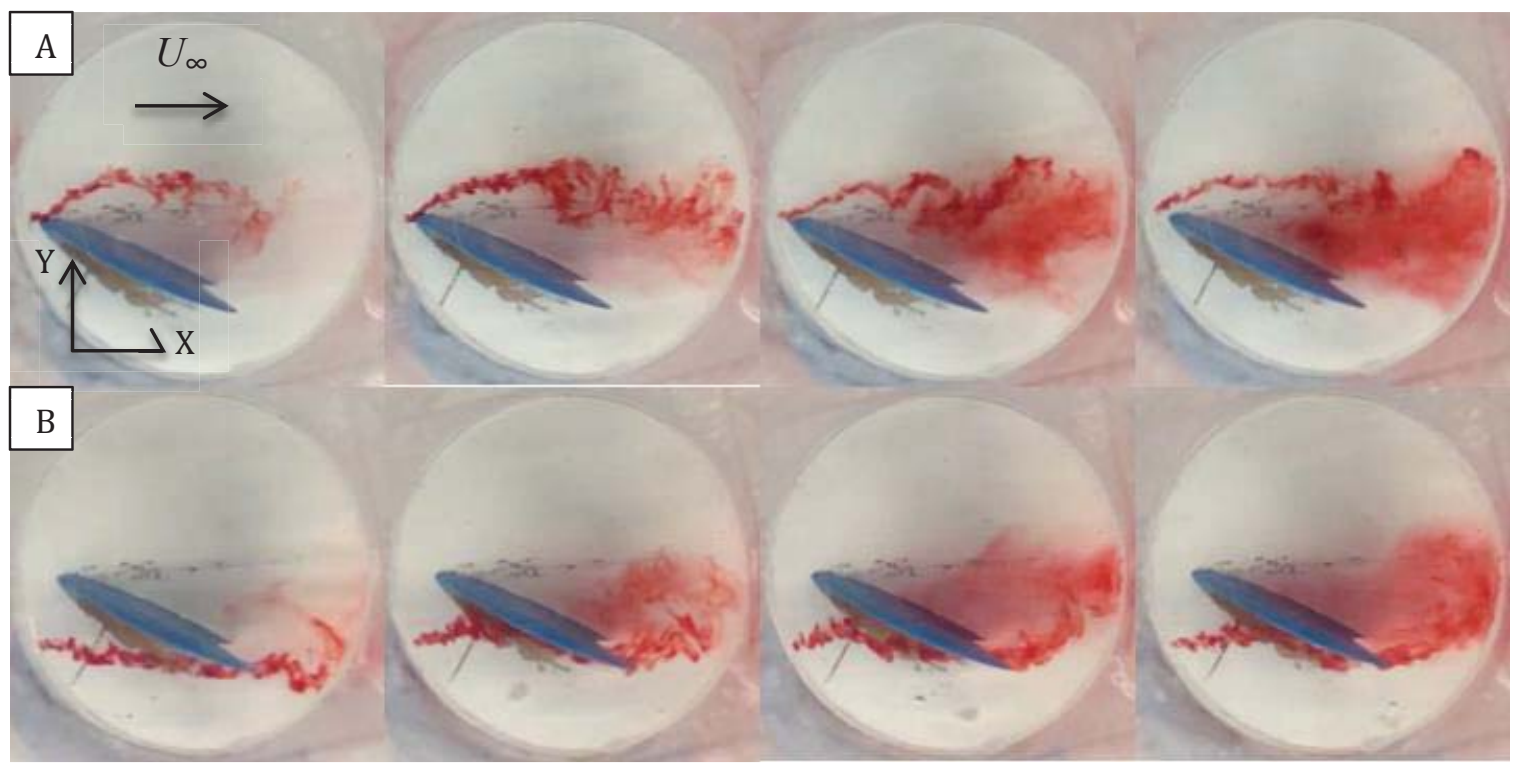

Figure 63: Plan view of the flow past the SFPW at $\alpha=25^{\circ}, U_{\infty}=0.5 \mathrm{mps}$ using dye injections at the base section (A) compression side. Sequence from left to right (B) suction side. Sequence from left to right.

The associated profile views are shown in Figure 64 and Figure 65. The dye injections on the compression side were shown in Figure 64. The first image showed some disturbance in the flow field, as the dye did not move in a straight path horizontally downstream of the fin. In the second image, the dye moved in a condensed straight path as it flowed past the leading edge, but then separated before reaching the trailing edge. Swirling eddies were identified in the third and fourth images, causing the dye to move in the vertical Z-direction from the base towards the tip of the fin. These images revealed the low velocity zone of dispersed dye expanding and extending in the downstream direction. 

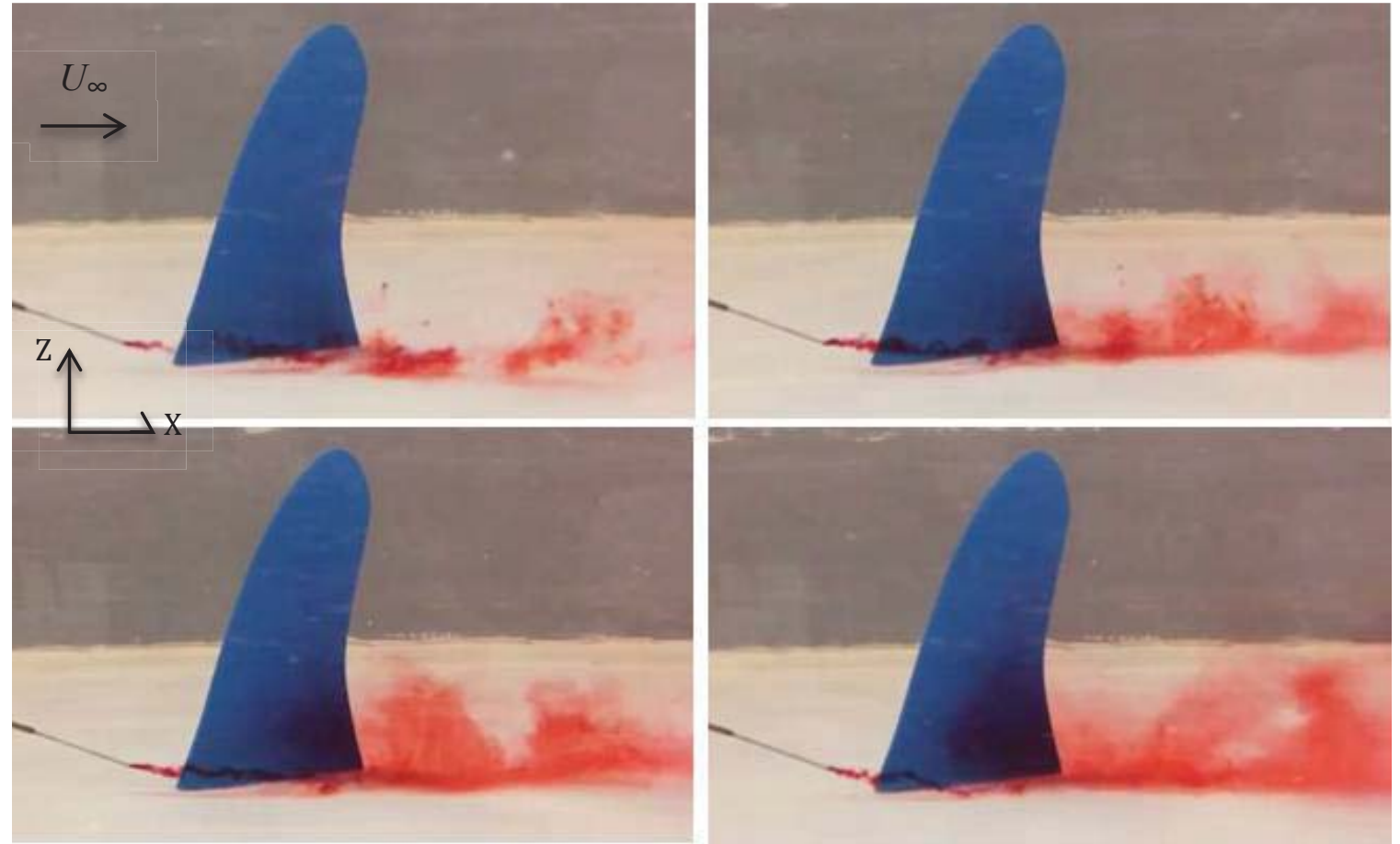

Figure 64: Profile view of the SFPW at $\alpha=25^{\circ}, U_{\infty}=0.5 \mathrm{mps}$ using dye injections on the compression side at the base section. Sequence from left to right.
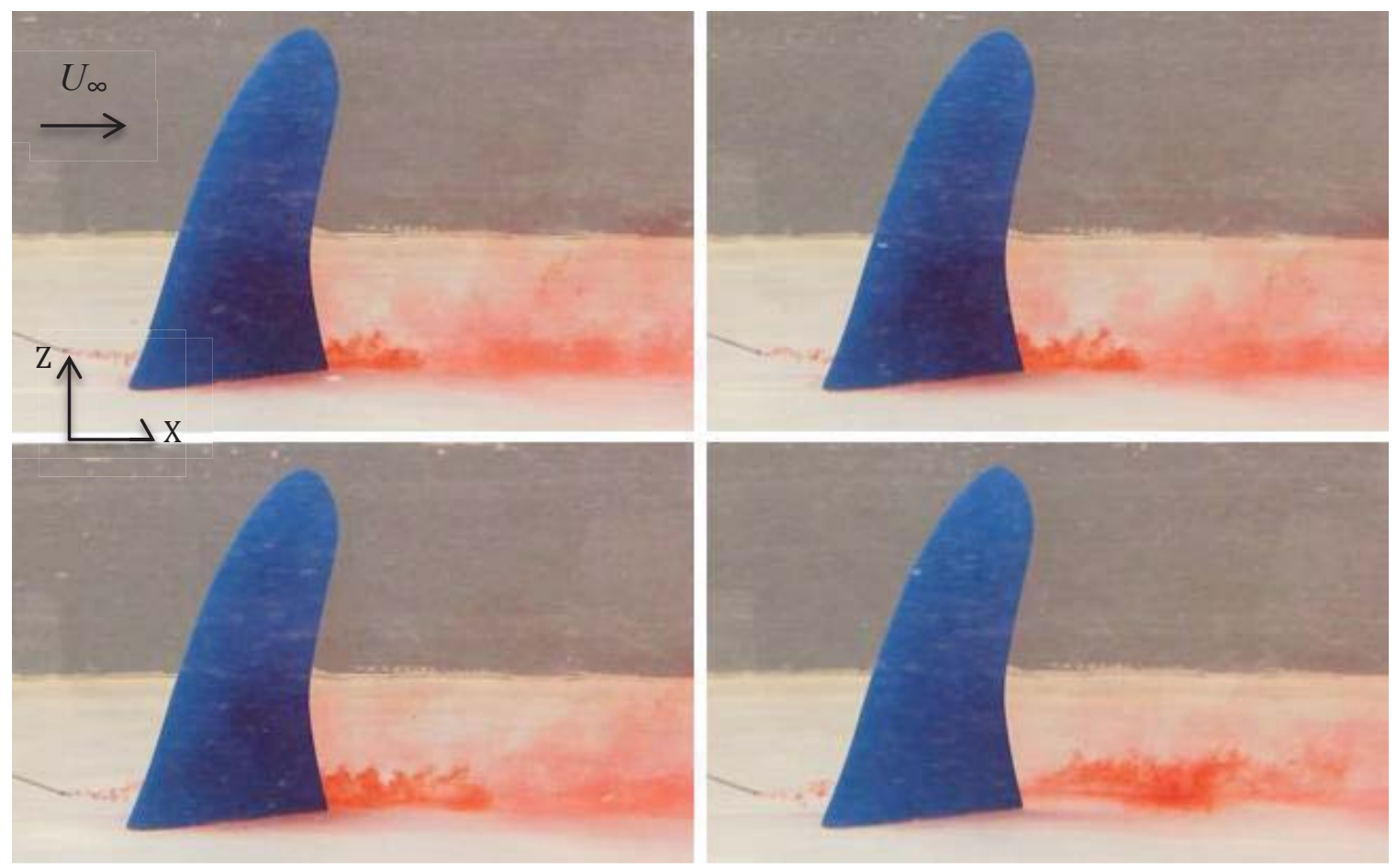

Figure 65: Profile view of the SFPW at $\alpha=25^{\circ}, U_{\infty}=0.5 \mathrm{mps}$ using dye injections on the suction side at the base section. Sequence from left to right. 
Figure 65 provides sequential images of the dye injections on the suction side of the SFPW fin. The first image indicated residual dye from prior injections moving over the compression side into the dead zone. Focusing near the trailing edge, dye can be seen moving around the tail of the fin. The second and third images show signs of turbulence, as the dye did not move in a straight laminar path in the X-direction downstream. The fourth image provides a visual of the dye curling towards the dead zone and movement in the vertical direction.

These same effects were found in the plan views in Figure 63. Both views display the dye moving in a condensed straight line past the leading edge and trailing edges, but dispersed as the fluid circulates within the dead zone. In the plan views, the zone of low velocity indicated circulation towards the fin and opposing the oncoming velocity direction. In the profile views, the swirling vortices moved in the vertical direction from the fin base towards the tip. This zone was also revealed expansion downstream in both views.

Dye was added at the mid-section for $25^{\circ}$ angle of attack, as shown in Figure 66. Figure 66(A) provides images of dye injections on the compression side. Similar flow patterns were seen at the mid-section, as compared to the base section. As dye flowed over the leading edge, it moved in a dense thin line. The dye did not stay attached to the fin's surface on the compression side. Immediately downstream of the leading edge, the dye began to separate and it circulated towards the surface of the fin. The swirling eddies were obvious in the second and third images. The area between the separated shear layers was considered 
the dead zone of low velocity. The dye remained fairly stagnant in this zone as the fluid continued to rotate towards the fin and deposit the dye.

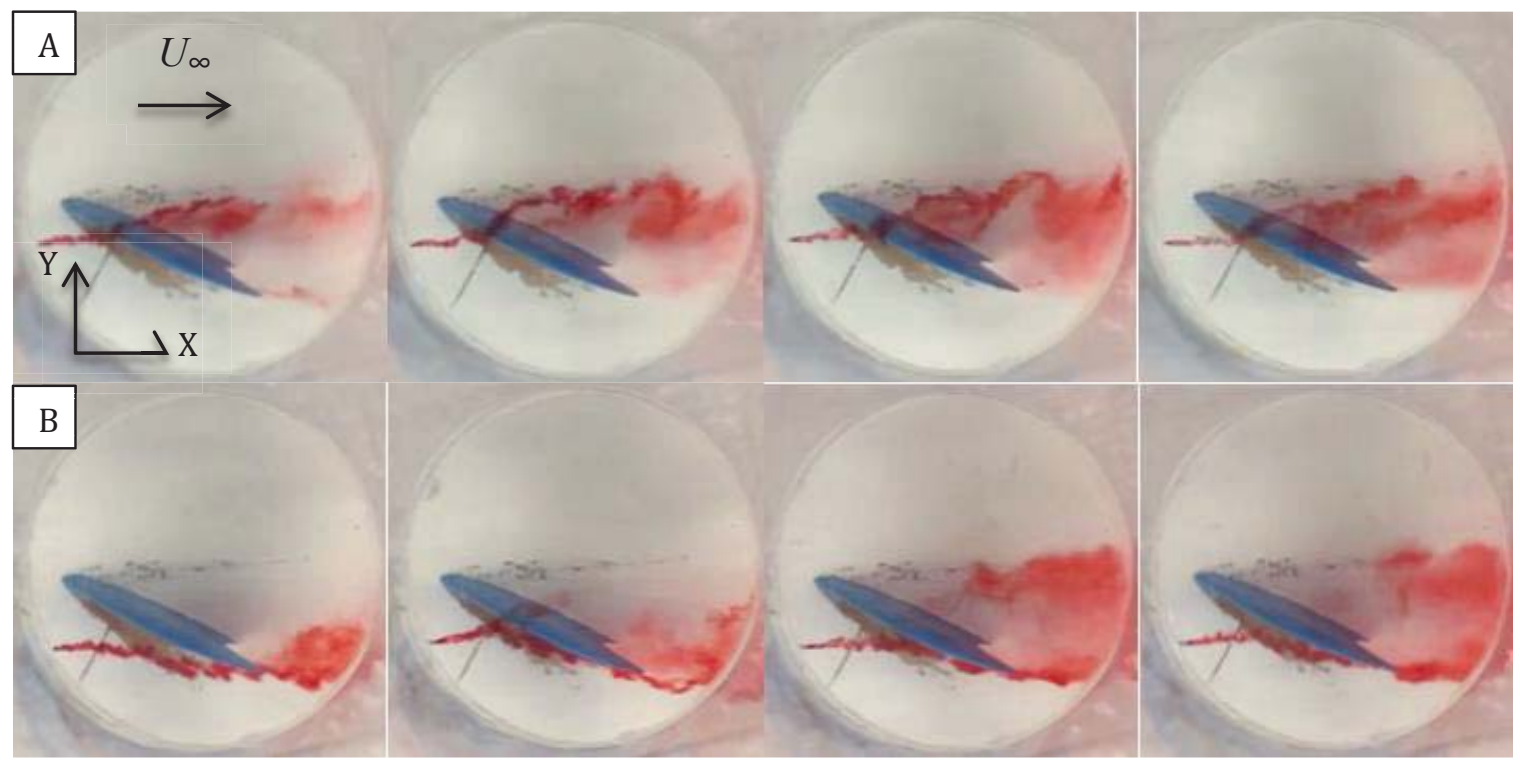

Figure 66: Plan view of the SFPW at $\alpha=25^{\circ}, U_{\infty}=0.5 \mathrm{mps}$ using dye injections at the mid-section (A) compression side. Sequence from left to right (B) suction side. Sequence from left to right.

In Figure 66(B), dye was added at the leading edge of the suction side of the fin. The dye did not make contact with the leading edge, but moved around it. Near the center of the fin, the dye connected to the surface of the fin until detachment at the trailing edge. Directly downstream of the fin's tail, the fluid circulated towards the upper surface. In the third and fourth images, the dye was shown to move in the opposite direction of oncoming flow. Here, the dye diluted and rotated within the dead zone area. 
Comparable effects were found in the profile views of the mid-section flow field. On the compression side of the fin, in Figure 67, the dye moved in the horizontal direction of flow before making contact with the leading edge. This dense, thin line dispersed directly after the leading edge. In the first image, the dye slightly shifted from the direction of flow and moved in a more turbulent manner downstream of the fin. These patterns intensified in the second image. By the third image, dye began to disperse in the vertical direction and showed more obvious signs of opposing flow rotation in the fourth image. These images were similar to the plan view, in which dye circulated within the dead zone in the X-and Y-directions. In the profile view, the dye circulated in this zone in the $\mathrm{X}$ - and Z-directions.
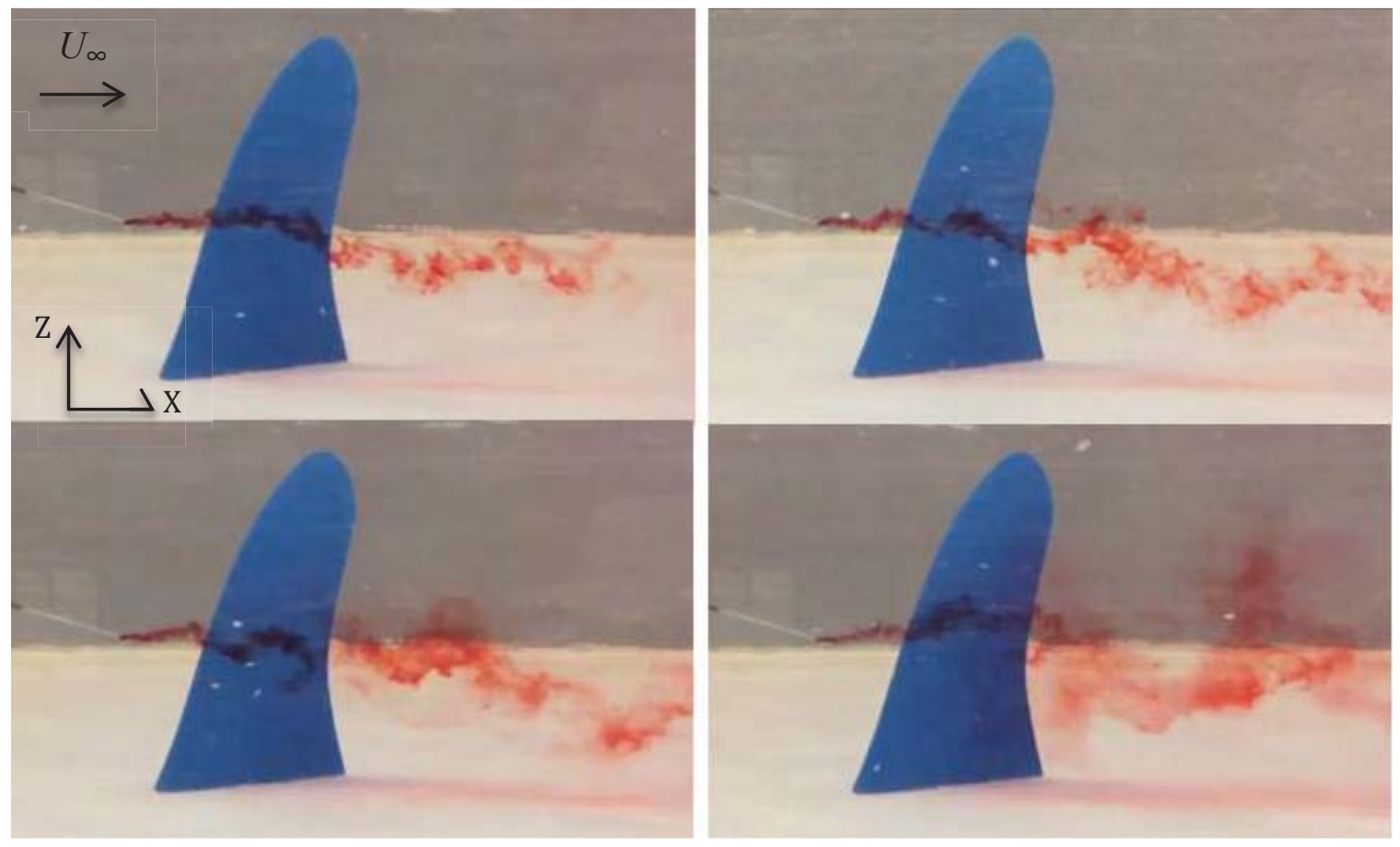

Figure 67: Profile view of the SFPW at $\alpha=25^{\circ}, U_{\infty}=0.5 \mathrm{mps}$ using dye injections on the compression side at the mid-section. Sequence from left to right. 
The suction side of the mid-section, in the profile view, is shown in Figure 68. The fluid demonstrated turbulent flow according to the chaotic patterns of the dye. After flowing past the trailing edge, the dye formulated into a more diluted state as it dispersed in the vertical Z-directions. This view, accompanied by the plan view, explained the movement of the fluid around the fin. After passing the trailing edge, the fluid rotated towards the dead zone area and moved in positive and negative Z-directions.
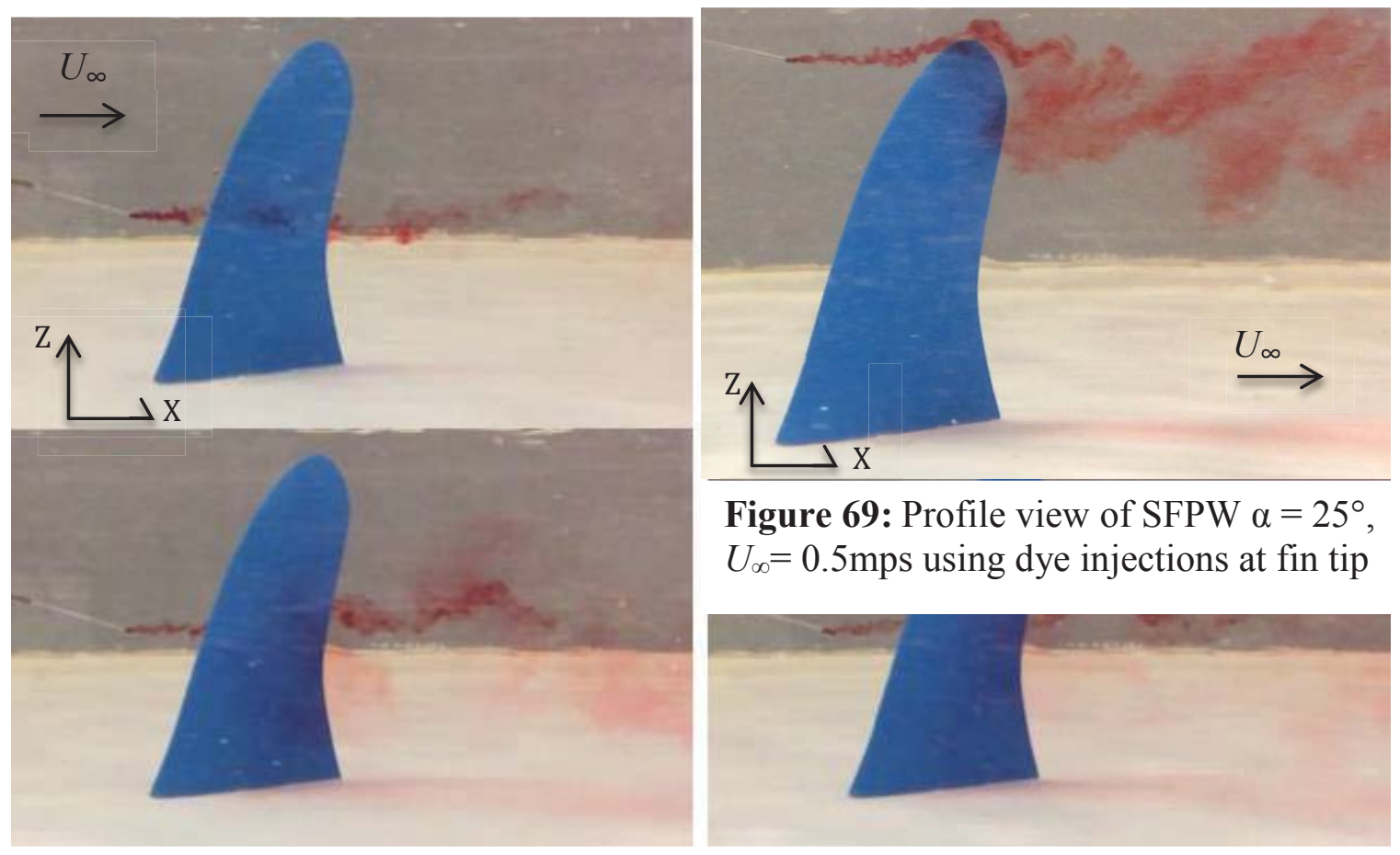

Figure 69: Profile view of SFPW $\alpha=25^{\circ}$, $U_{\infty}=0.5 \mathrm{mps}$ using dye injections at fin tip

Figure 68: Profile view of the SFPW at $\alpha=25^{\circ}, U_{\infty}=0.5 \mathrm{mps}$ using dye injections on the suction side at the mid-section. Sequence from left to right.

While the fin remained at $25^{\circ}$ angle of attack, dye was inserted near the tip. The dye flowed in a thin horizontal line before reaching the leading edge. The dye moved over the tip and then immediately dipped in the negative Z-direction. In this area, the dye began to disperse. The flow circulated downwards toward the mid-section of the fin. Tip vortices were evident in the trailing wake of the fin. 
At $45^{\circ}$ angle of attack, drastic changes in the flow field were seen when compared to the previous incident angles. Plan views of the dye injections at the base sections are presented in Figure 70. The sequence of dye moving on the compression side is shown in Figure 70(A). The dye moved in a condensed line around the leading edge, but scattered as it swirled into the dead zone area. The video showed the dye flowing at a higher velocity as it passed the leading edge. The velocity rapidly decreased immediately downstream of hydrofoil's front edge. Opposing the oncoming flow, the dye circulated back towards the leading edge. Small vortices are shown in all four images depositing dye into the dead zone. Due to the lack of fluid movement, dye was absorbed in this zone. It took several seconds for the dye to completely dilute downstream once injections stopped. The dye took longer to reduce at this angle compared to smaller angles of attack with flow separation. Furthermore, the dead zone area was larger than the other angles measured.
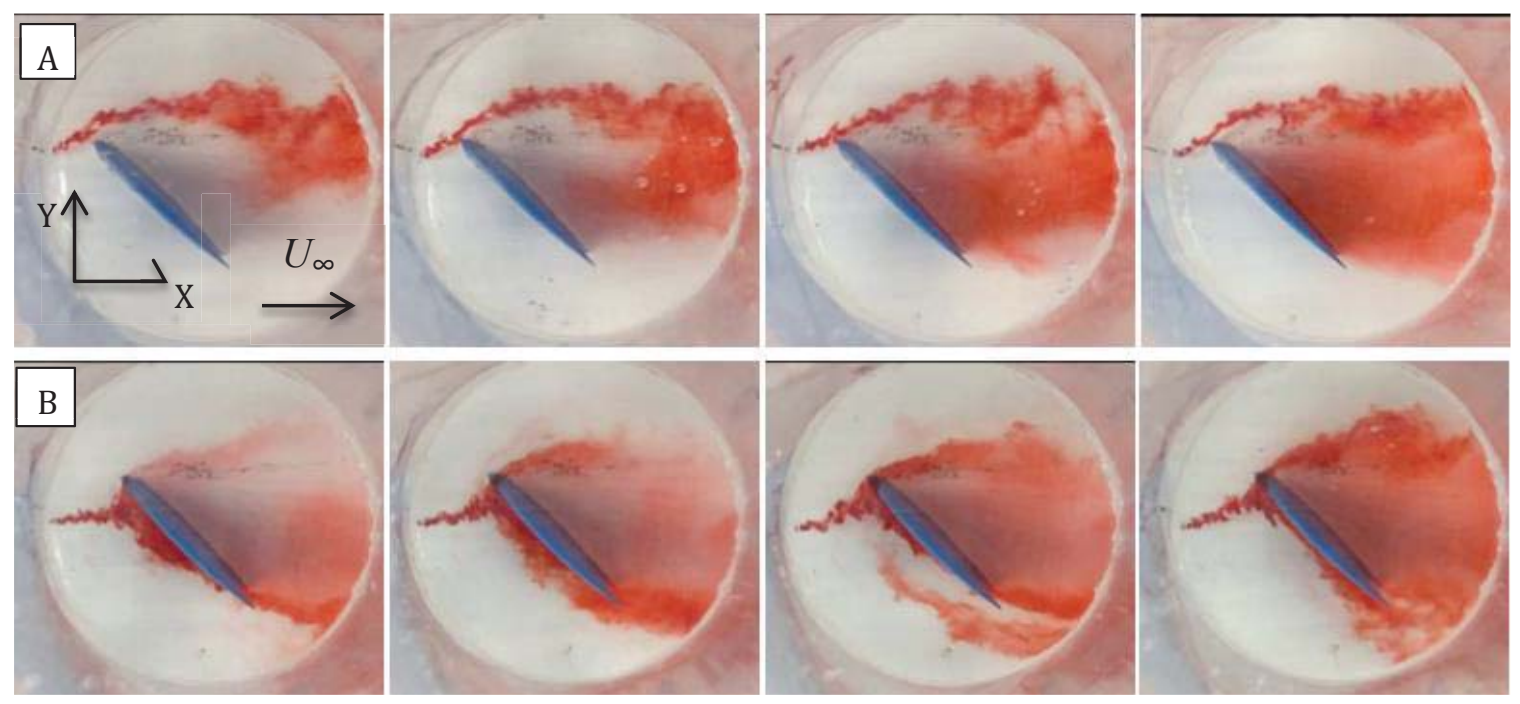

Figure 70: Plan view of the SFPW at $\alpha=45^{\circ}, U_{\infty}=0.5 \mathrm{mps}$ using dye injections at the base section (A) compression side. Sequence from left to right (B) suction side. Sequence from left to right.

Figure 70(B) illustrates the dye injections at the lower surface. As dye was added near the suction side of the leading edge, it easily slipped to the upper surface in many of these images. The dye stayed attached to the fin from the leading to trailing edge on the suction side. As the dye detached from tail, it dispersed and circulated towards the dead zone. The circulating vortices moved fluid in the opposing direction of the oncoming flow velocity. 
The video provides evidence of the dye moving around the fin and velocity differences were seen. Near the leading edge, the dye moved slower in velocity, but sped up as it reached the tail of the fin. Low velocities were found in the dead zone area, where dye was deposited. Not as much dye was added from the suction side as compared to the compression side.

The profile views of the dye injections at the compression and suction sides are presented in Figure 71 and Figure 72, respectively. The first image in Figure 71 shows the dye moving around the leading edge in a thin compressed manner. It separated as soon as the flow passed over the fin's surface. Directly downstream of the fin, the flow turned back towards the fin. These swirling vortices caused the flow to move opposite of the oncoming flow and deposit dye into the dead zone. As the vortices spun near the base of the fin, the flow in the trailing wake also circulated in the positive Z-direction. This zone extended a greater distance in the X-direction as compared to the smaller measured angles of attack. The dye

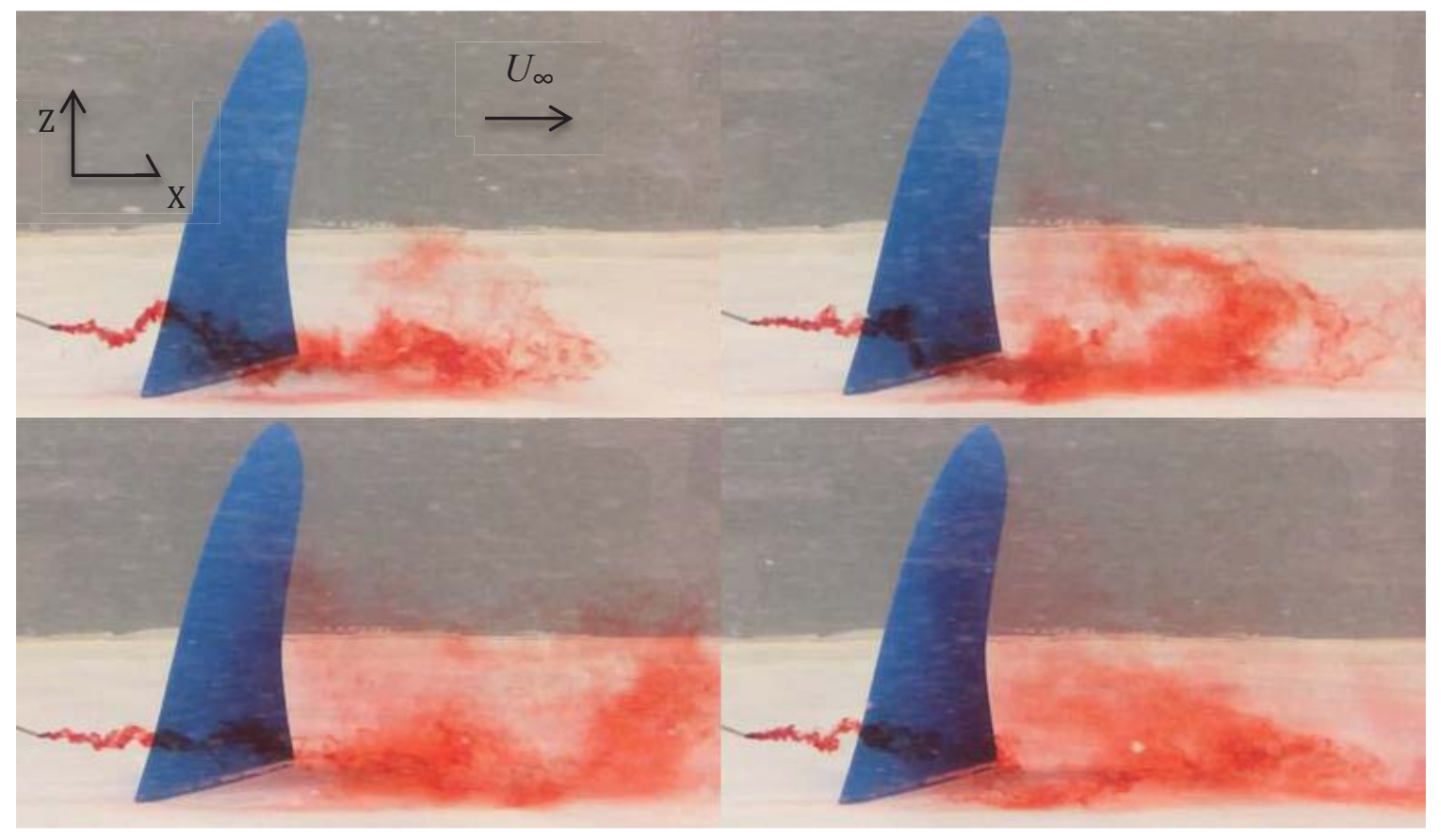

Figure 71: Profile view of the SFPW at $\alpha=45^{\circ}, U_{\infty}=0.5 \mathrm{mps}$ using dye injections on the compression side at the base section. Sequence from left to right.

also showed movements that were in accordance to turbulent flow in the water channel. The profile views corresponded to the flow field shown in the profile view in Figure 70(A). 
In Figure 72, the dye moved around the suction side of the fin. Turbulence was still evident in the water channel. As the flowed past the trailing edge, it showed dispersion and movement in the positive Z-direction. In the second and third images, flow was shown to move into the dead zone and circulate there. This zone of low velocity displayed expansion in the positive X-and Z- directions as dye accumulated. Comparing these images to the plan view, in Figure 70(B), it was found that the dye moved over the surface of the fin in a more condensed line and then dispersed once it detached from the trailing edge. The flow patterns were complimentary for the plan and profile views.

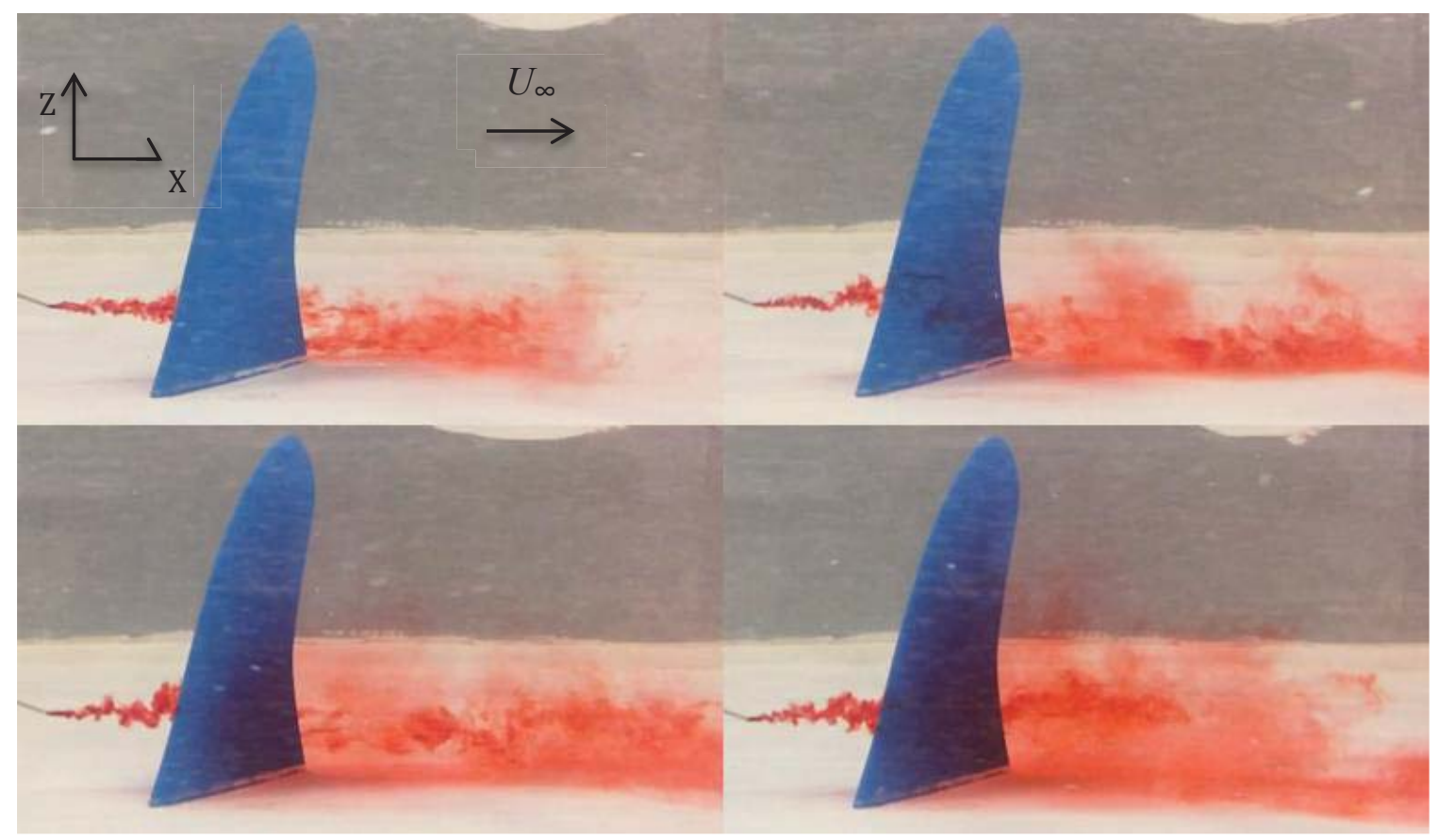

Figure 72: Profile view of the SFPW at $\alpha=45^{\circ}, U_{\infty}=0.5 \mathrm{mps}$ using dye injections on the suction side at the base section. Sequence from left to right.

The mid-section views produced similar flow field patterns. Figure 73 provides the plan views of dye injections at the mid-section of the fin for the upper and lower surfaces. In Figure 73(A), the dye is added on the compression side of the fin. Once again, the dye was shown to move at a higher velocity in a thin, condense line around the leading edge. Immediately downstream of this, the dye formed swirling eddies that deposited dye into the dead zone. Figure 73(B) also showed the dye depositing dye into this zone from the fin's tail edge trailing vortices. The dye was found to circulate within the low velocity area. 

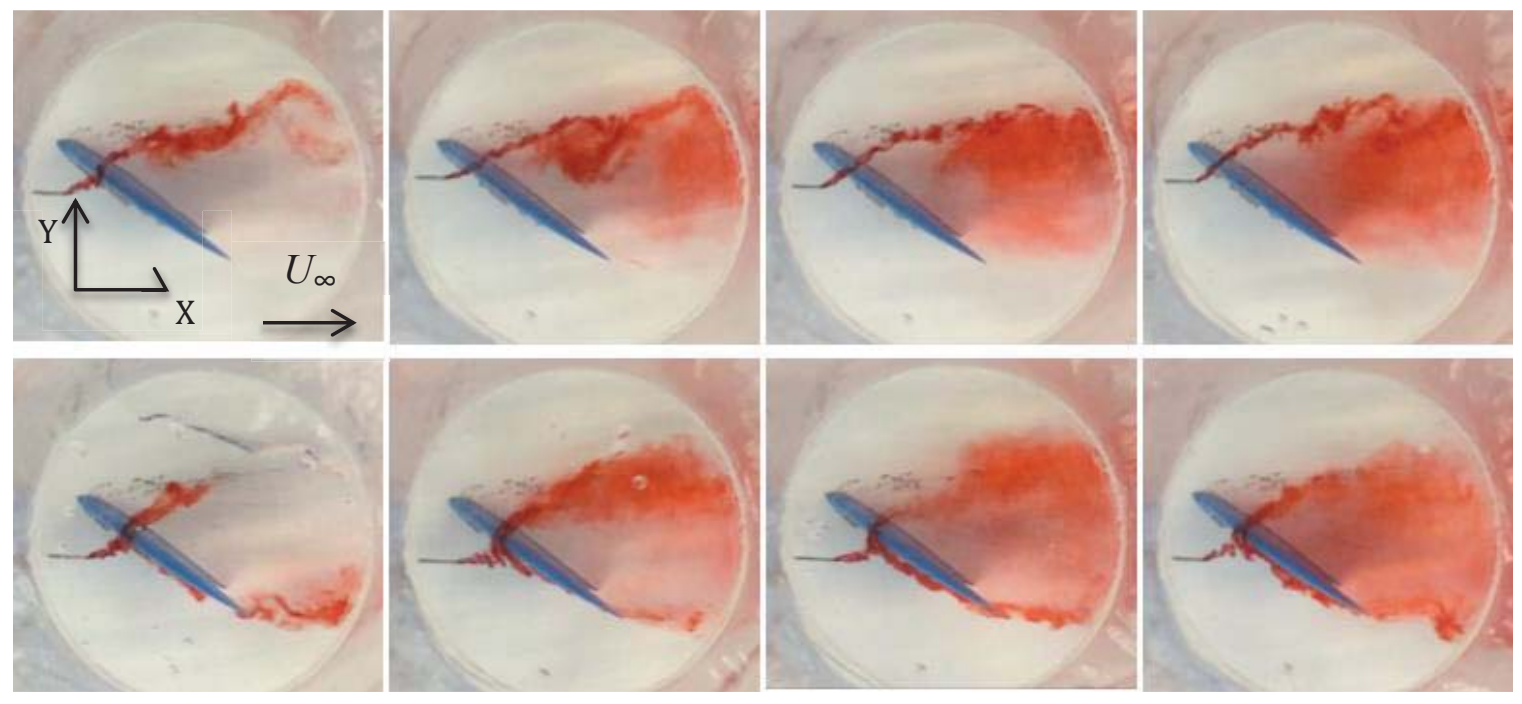

Figure 73: Plan view of the SFPW at $\alpha=45^{\circ}, U_{\infty}=0.5 \mathrm{mps}$ using dye injections at the mid-section (A) compression side. Sequence from left to right (B) suction side. Sequence from left to right.

The accompanying mid-section profile views are presented in Figure 74 and Figure 75 . In Figure 74, the dye moved at a higher velocity over the leading edge of the fin's compression side. The velocity decreased and dye separated immediately following the departure of the leading edge. The dye and fluid appeared to rotate behind the fin, opposing the oncoming flow direction. It accrued within the zone of low velocity and spread in the positive and negative Z-directions. These swirling vortices and dye accumulations were also seen in the plan view images of Figure 70(A).

Figure 75 provides the addition of dye on the suction side. Dye slipped to the compression side due to pressure and velocity differences. Once the dye moved passed the trailing edge, it supplied dye to the dead zone. The dye in this area moved vertically towards the base and tip of the fin. High amounts of turbulence were seen in these images, as the dye did not follow a common path or trend throughout the video. 


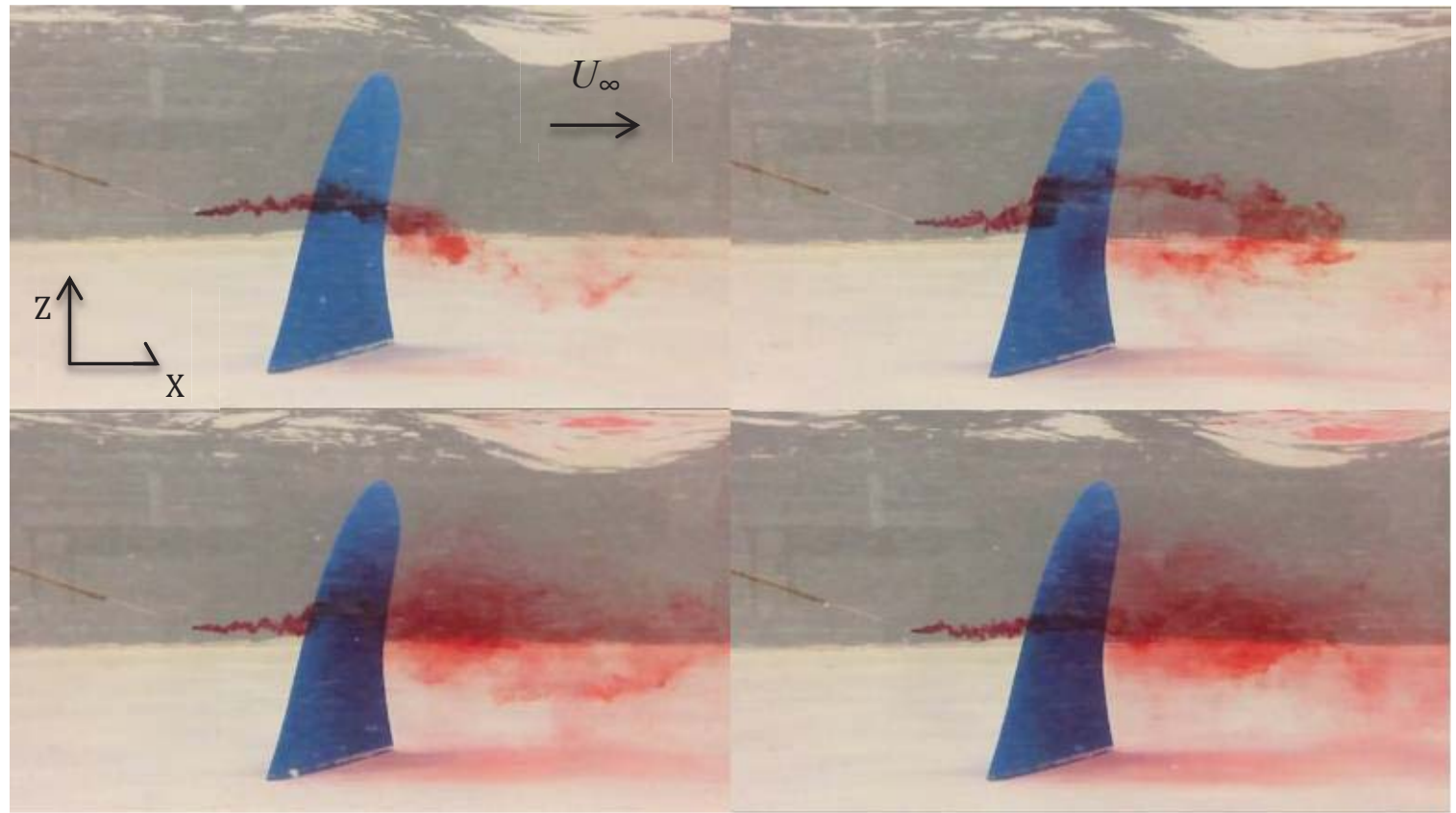

Figure 74: Profile view of the SFPW at $\alpha=45^{\circ}, U_{\infty}=0.5 \mathrm{mps}$ using dye injections on the compression side at the mid-section. Sequence from left to right.

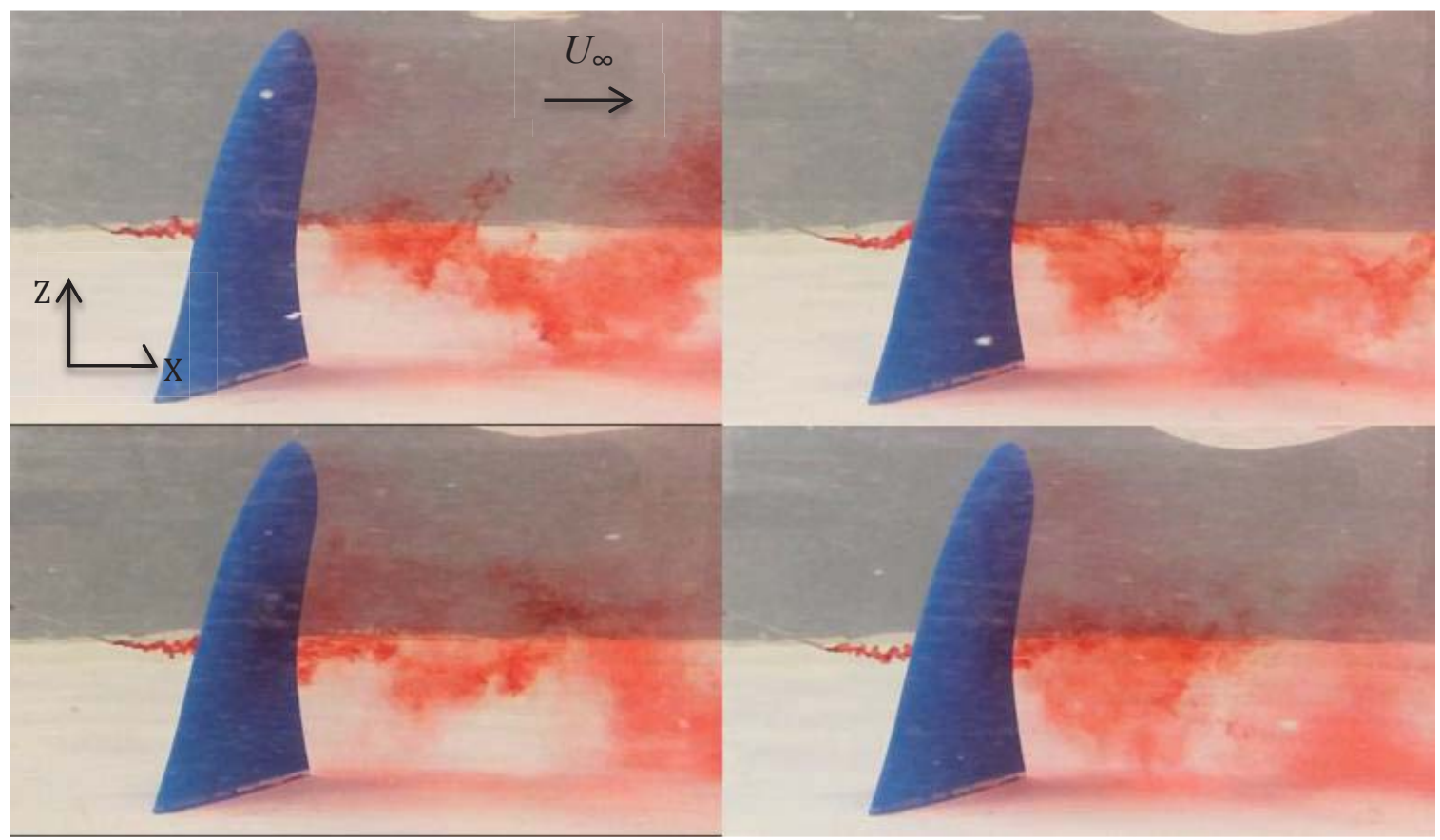

Figure 75: Profile view of the SFPW at $\alpha=45^{\circ}, U_{\infty}=0.5 \mathrm{mps}$ using dye injections on the suction side at the mid-section. Sequence from left to right. 
In Figure 76 , the fin tip at $45^{\circ}$ angle of attack provided similar dramatic effects, as compared to the incident angle $25^{\circ}$. The flow moved in a condense line over the tip, but dispersed as soon as it detached from the fin surface. The fluid dipped below the dye insertion level and circulated in the clockwise direction towards the fin. This dye also deposited dye into the dead zone area.

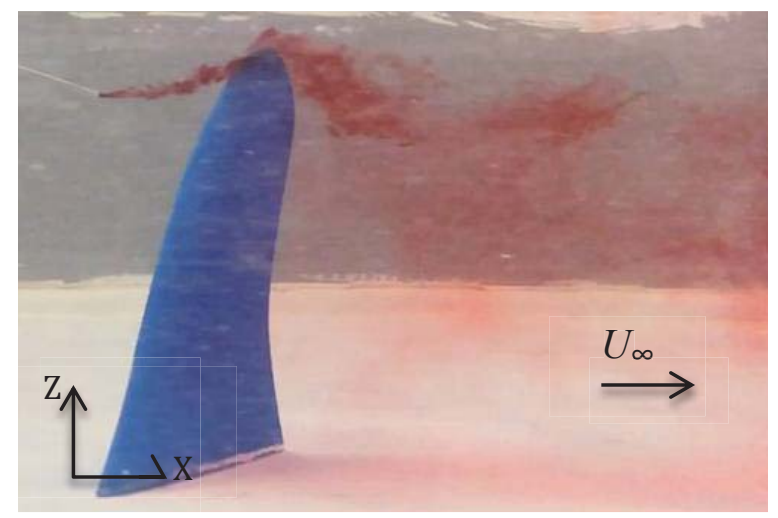

Figure 76: Profile view of the SFPW at $\alpha=45^{\circ}, U_{\infty}=0.5 \mathrm{mps}$ using dye injections at the fin tip 


\subsection{DISCUSSION}

\subsubsection{RESULT COMPARISONS}

Based on lift and drag coefficient curves for two-dimensional airfoils, the fin results provided similar curve shapes. The experimental lift coefficient plots for a twodimensional NACA0012 airfoil, presented by NASA, were higher in value because the associated Reynolds number was significantly higher (Rumsey, 2014). The lift coefficient curves were similar, following the same patterns at each angle of attack. These commonalities were expected based on finite wing theory (Anderson, 2005). The finite three-dimensional fins, designed in this thesis, were made up of many two-dimensional NACA0012 hydrofoils. Because the fin has more surface area than a 2D hydrofoil, values of higher skin friction and pressure drag due to flow separation would transpire. Tip vortices and resulting downwash would also contribute to lower lift coefficients and higher drag coefficients than compared to the two-dimensional NACA0012 hydrofoil.

The lift and drag coefficients were lower than the single fin results gathered by Carswell and Lavery (2006). This would be contributed to the differences in fin geometry, oncoming velocitie values, and turbulence intensities. The shapes of the curves were dissimilar because the range of incident angles were not the same. Carswell and Lavery (2006) focused on smaller angles of attack in $2^{\circ}$ increments. This level of accuracy could provide small local bends in the more broad lift and drag curvature, which was presented in this thesis.

As stated earlier in the report, differences between the CFD and laboratory results could be due to imprecise turbulence intensities, inaccurate data collection devices, and/or the experimental lab setup. The experimental lift coefficient results deviated by an average of $31 \%$ from the CFD findings. The drag coefficients differed by an average of $26 \%$. These might be considered large errors, but there are several factors that must be considered. The size geometry of the fins differ, which could have slightly effected the lift and drag values in the experimental study. The turbulence formed in the water channel might have been higher or lower than the $4 \%$ turbulence intensity set for the NX FLOW simulator, which could have provided some differences between the values. Although the surfboard lift and 
drag values were subtracted from the final results, the surfboard was not present in the CFD methodology. The surfboard may have altered the flow field effects near the base of the fin, causing generally higher lift and drag coefficient values.

In addition, most of the error was found at $0^{\circ}$ angle of attack. Most fins produced less than $25 \%$ error, between the two methods for the lift coefficients, at all angles higher than $0^{\circ}$, except the SD, FW, SF, and M. The SD and Marlin calculated greater than 25\% differences at $0^{\circ}$ and $5^{\circ}$. The $\mathrm{FW}$ and SF had high variance in values at $0^{\circ}$ through $10^{\circ}$. If these high deviations were to be omitted, there would only be an average $8 \%$ difference between the CFD and laboratory lift coefficient values. The spring gauges produced high sources of error due to human and device influences. The value differences at $0^{\circ}$ angle of attack were extremely small and could be contributed to the data collection method completed in the lab. The highest lift coefficient value difference, at $0^{\circ}$, was found to be 0.05 , which is fairly small when considering lift. This calculated a $189 \%$ variation between the CFD and lab results for the Marlin at $0^{\circ}$.

The SFPW deviated 25\% between the CFD and lab results for the lift coefficients, but yielded only an $8.6 \%$ difference when ignoring $0^{\circ}$ incident angle values. The drag coefficients were even closer in value compared to the lift coefficients for the SFPW. The highest value gap was found to be 0.11 at $25^{\circ}$, generating an $18.3 \%$ differentiation. The greatest percent variation, of $37 \%$, was shown at $5^{\circ}$. Averaging the differences between the two methods for all ten angles of attack, it was revealed that there was only a $12.8 \%$ gap between the CFD and lab results for the drag coefficients. This was the lowest for all nine fins. The average percent variation, for the SFPW's lift coefficients, was also the lowest for all nine fins.

\subsubsection{OPTIMUM FIN DISCUSSION}

The SFPW was chosen as the optimum fin based on the results from the laboratory and the computational outcomes. The CFD results revealed that this fin produced the highest lift forces at all angles of attack and had the most commonly occurring maximum L/D ratio measurements. For typical surfing conditions, high L/D ratios at small angles of attack give the surfer ability to maneuver easily and greater speeds. Within that the suitable incident 
angle range, stated by Lavery et al. (2009), the SFPW produced high L/D ratios. At $10^{\circ}$, the SFPW had the peak L/D ratio value among all the fins for the ten angles of attack. The laboratory results also shared paralleled success. Eight out of the ten angles of attack, the SFPW yielded the highest lift forces. Between $15^{\circ}$ and $20^{\circ}$, this fin had the greatest L/D ratios of the nine fins. It also had the most commonly occurring high $\mathrm{L} / \mathrm{D}$ ratio values compared to the other eight nine fins. The SFPW was one of the most efficient fins in the smaller incident angles.

There was not a significant difference between the lift coefficients for the CFD and lab results. For almost all angles of attack, the lab results produced slightly higher lift coefficient values. The drag coefficients are closely related. At smaller angles of incidents the gap between the two methods is slightly higher than the values at higher angles of attack. The quantitative differences in these results could have been forewarning to the variances in the visual flow field analysis.

\subsubsection{HIGH VELOCITY DISCUSSION}

The high velocity values were obtained using the CFD method in order to compare the lift and drag coefficients and to find the lift and drag forces at typical surfing velocities. It was found that there were small ranges of error between the coefficient results, and the differences magnified as the velocities increased. At smaller angles of attack, the differences were more extreme. Because the variances between the high velocity values and the $0.5 \mathrm{mps}$ values were fairly insignificant, lift and drag coefficient equations could be used in the future to predict approximate lift and drag force values for these particular fins using the CFD results. It must be understood that there would be some margin of error included in these results.

\subsubsection{CFD VISUAL DISCUSSION}

The SFPW was the optimum fin used for the visual investigation of the flow field. The four chosen angles of attack were analyzed and discussed in detail in the following paragraphs. Comparisons were made between the laboratory images and the CFD images. Conclusions were drawn in order to provide suggestions into why differences may have occurred between the two methods. 
The four angles of attack produced typical flow patterns for the finite fin with a symmetric hydrofoil shape. At $0^{\circ}$ angle of attack, the streamlines were shown to stay attached to the fin's surface. Similar flow effects were shown at the base and mid-section. Proven by experimentation and theory, no separation occurred within the flow boundary layer structure. Equivalent velocity magnitudes and symmetric fluid movements were produced on the upper and lower surfaces of the NACA0012 hydrofoil shape, when the twodimensional plan view was investigated. Because the fin allowed for even water flow on both surfaces, the fluid pressure and directional velocities were identical at the point of contact following the trailing edge. Because of this, and the finite angle of the trailing edge, the fluid left the trailing edge smoothly, without signs of circulation (Anderson, 2005). Based on the Kutta condition, two stagnation points existed at two points. As expected, these included the leading edge, which was the lead surface perpendicular to the oncoming streamlines, and the sharply pointed trailing edge (Kundu \& Cohen, 2008).

The fluid moved three-dimensionally, so the profile view was also examined. The fin is really just a set of stacked hydrofoils with a finite length. This provided even water flow in the direction of the oncoming fluid vectors across the length of the fin.

Lifting values at $10^{\circ}$ were greater than the values produced for $0^{\circ}$ incident angle, which was shown in Figure 23. This was due to the repositioning of the pressure and velocity gradients. At $0^{\circ}$ angle of attack, the area of highest pressure was at the leading edge because it was the initial point of contact from the oncoming flow. When the angle of attack increased to $10^{\circ}$, this point of direct impact moved slightly below the leading edge. This area received high positive pressure values, while simultaneously causing water velocity values to plummet. The increased incident angle caused the two stagnation points to change position along the fin's surface. The first stagnation point moved below the leading edge in the area of low velocity (Kundu \& Cohen, 2008). The rear stagnation point moved on the top surface near the trailing edge. Here, the top surface's adverse pressure gradient was positive and velocity values were low. Because the $\mathrm{dp} / \mathrm{dx}$ was small, flow did not separate from the fin's surface (Anderson, 2005). This was in accordance with the Kutta condition theory (Anderson, 2005). Concurrently, low negative pressure values were formed on the 
fin's top surface, near the leading edge (Kundu \& Cohen, 2008). The pressure changes were due to the Bernoulli's effect.

As the fin's angle of attack increased to $10^{\circ}$, the streamlines were compressed on the top surface, near the leading edge, and on the bottom surface, near the trailing edge; Figure 33 and Figure 35. This caused decreases in pressure at both of these areas, as shown in Figure 34. These low pressures allowed for higher velocities over the leading edge on the top surface and near the trailing edge on the bottom surface. The increased velocity magnitudes, at the leading edge, were shown for the length of the fin in Figure 36. As expected, some signs of tip vortices were present in the streamlines in Figure 35. These tip vortices were produced by water flowing over the top of the angled fin, from a high to a low-pressure side (Anderson, 2005). This movement produced circulatory motion downstream of the fin. Because the flow was attached at $10^{\circ}$, pressure differences were not extreme. Because of this, tip vortices were also not intense.

Attached flow was still present at $25^{\circ}$ angle of attack. Based on the lift coefficient curve, in Figure 23, separation was not expected. This was because lift coefficients increased in value from the previous angle of attack, and signs of stall occurred after $25^{\circ}$. At $30^{\circ}$ incident angle, lift coefficient values decreased by $2.7 \%$ from the values at $25^{\circ}$. Major signs of flow separation are typically seen after the critical angle of attack, causing the fin to stall and lose lift. The lift coefficients for the CFD results peaked at $25^{\circ}$ angle of attack, electing it the critical angle of attack.

Both Figure 39 and Figure 41 show fluid attachment as flow moves over the fin at the base and mid-section regions. The differences in velocity and pressure magnitudes provided evidence to the cause of lift. The decreases in velocity were accompanied by increases in pressure. These pressure increases were found at the bottom side at the leading edge and on the topside at the trailing edge, as shown in Figure 40. The decreases in pressure on the topside of the leading edge caused vast pressure differences around the fin. The velocity increases and pressure decreases were located at the leading edge across the length of the fin in Figure 42. In Figure 43, the velocity decreases were located across the length of the fin on the bottom side. An object will move to lower pressure areas as the higher-pressure 
systems provide the energy to move it there. These pressure differences along the fin length provided a lifting action. The differences in pressure between the upper and lower surfaces were greatest at $25^{\circ}$, allowing for the highest amount of lift force of the ten angles of attack investigated. This optimum angle of attack for the lift forces was identified at $25^{\circ}$ for all nine fins in the CFD results.

The reduction in lift coefficients, after the critical angle, as angle of attack increases was identified as stall. At $45^{\circ}$, the lowest lift coefficient values, of the ten investigated angles of attack, were produced. This was shown in Figure 23 and was further explained through the visualization of the flow field at $45^{\circ}$. The fin position was no longer streamlined, causing detrimental disruptions in the flow and lifting components. High-pressure values were seen across the suction side of the fin in Figure 46. Flow boundary separation caused two shear layers that shed from the leading and trailing edges of the fin. Between these shear layers was a dead zone that had extremely low velocity values and high negative pressure values. These low velocity values were shown in blue in Figure 45 and Figure 47. The separation of the fluid boundary layers induced drag, which reduced the fin's lift. As angles of attack increase, the flow separation and the dead zone area become larger. This causes more drag, which decreases the lift forces. The highest angle of attack investigated in this thesis was $45^{\circ}$. Here, flow separation was present and provided the largest dead zone area of all ten angle. Within the dead zone area, vortices were discovered. The trailing vortices swirled and moved in the opposite direction of the oncoming flow, which induced drag and helped reduce the lift forces. These vortices are shown to move towards the fin tip and base section along the length of the fin in Figure 47 and Figure 49.

\subsubsection{LAB VISUAL DISCUSSION}

Figure 51 and Figure 52 showed the symmetric fin at $0^{\circ}$ angle of attack. This allowed for even water flow around both sides of the fin. According to the CFD plan view images at $0^{\circ}$, the streamlines and flow vectors present horizontal flow. The fin produces almost no disturbance in the direction of flow. The water smoothly runs over the surface of the fin and the flow vectors do not show any shedding vortices. Increases in velocity were found on the upper and lower surfaces of the hydrofoil. Decreases in velocity were found at the leading and trailing edges. In the laboratory video evidence, it is difficult to see any changes 
in velocity around the surfaces of the fin. This was true for all sections and views of the SFPW at $0^{\circ}$ angle of attack. In Figure 51, the third and fourth image did show the dye was thinner directly behind the trailing edge. This could be a result of decreased velocity, which was seen in the CFD results. The laboratory images provide comparable flow patterns for the plan view, at the base and mid-section of the fin. Although the dye showed the expected horizontal movement in the X-direction at the fin's mid-section, it did appear there were small eddies or signs of turbulence. This could have been caused by the injection process or higher turbulence in the water channel than expected. Shedding vortices were not evident in the plan view images. The CFD images showed the flow vectors producing horizontal flow patterns, without any movement in the Y-direction.

Comparing the profile views of the CFD and lab images at $0^{\circ}$, small differences were found. The CFD profile images delivered streamlines and flow vectors with horizontal flow movements. There was a slight increase in velocity as the flow expanded around the widest section of the fin. This velocity increase was difficult to detect in the lab images and video. The lab results also displayed some vertical movement. Trailing vortices appeared downstream of the fin. These disturbances could have been eddies and shedding vortices from the trailing edge, or natural turbulence effects from the water channel. The dye injection process could have also caused the fluxes in the vertical direction. This would have meant vortices were not natural, but human-induced.

Due to differences in the injection technique, dye did not inject exactly the same for all sections. There was slightly more turbulence at the mid-section of the fin compared to the base section. This could be from the boundary effects of the water channel's bed. It also could be caused by the dye-injector applying different injection techniques.

Inspecting the plan view at $10^{\circ}$ angle of attack, the dye moved smoothly around the base of the fin without any flow separation. The CFD images showed an increase in velocity near the leading edge on the compression side. This caused the flow lines to converge. Near the trailing edge, the streamlines spread out to continue to move in the horizontal direction downstream. From the video, it can be seen that there is a slight increase in velocity near the leading edge on compression side. This could be the reason the dye runs in a thinner 
more condensed line around this compressed edge. Due to the decrease in velocity near the trailing edge, the dye disperses causing a thicker layer of dye. On the suction side, it was more difficult to place the nozzle at the leading edge without the dye moving to the compression side. This was because there was such a low velocity, and high pressure, in this area that the dye could easily move to the top surface. As discussed in Section 1.5.4, a fluid can easily move from high to lower pressure areas. These two high and low pressure areas were adjacent to one another, so the dye naturally wanted to move to the area of lower pressure. The dye did not show any changes in density thickness from the tip to the tail on the suction side. In the CFD images, there was a decrease in velocity at the leading edge of the suction side. The proximity of the streamlines, on this side, was well distributed at the low velocity leading edge. The streamlines compressed slightly closer in proximity to one another near the trailing edge.

Focusing on the plan view, the mid-section in the laboratory results showed similar effects to the CFD results at $10^{\circ}$ angle of attack. From the CFD data, the flow vectors stayed attached to the fin on both the upper and lower sides of the hydrofoil; as shown in Figure 33. At the leading edge of the compression side, there was an increase in velocity and higher density of vector arrows. At the tail of the foil, the flow spread out and was not as concentrated. This was seen with the dye in the lab images in Figure 56(A). The dye was more compressed at the leading edge and then dispersed towards the trailing edge. On the suction side, the velocity decreased at the leading edge, as found in Figure 56(B). The vector arrows in Figure 33 had a constant spacing arrangement, until the trailing edge where the vectors became more compact. The lack of dye at the leading edge, of the lab results, could be evidence of this decrease in velocity. Unlike the CFD results at the trailing edge, the dye seems to disperse more near the tail of the fin. The dye being injected into the water moved in a wavy-turbulent manner. This turbulence was more apparent at the mid-section and could be caused by the injection process, human mistake, or turbulence in the water channel.

Corresponding flow patterns were seen in the profile view of the lab results for both the compression and suction sides in Figure 60 and Figure 61, respectively. The difference 
between the CFD and lab data was found in the profile view. After the trailing edge, the lab images showed disturbance with small movements in the Z-direction. The CFD results in Figure 37 showed the flow vectors moving horizontally in the X-direction, without any inclinations towards the Z-directions. This could be due to higher turbulence in the water channel compared to the applied turbulence in the NX-FLOW inputs.

At $25^{\circ}$ angle of attack, some signs of flow separation were discovered in the lab results of Figure 63 through Figure 69. Comparing the laboratory plan views, in Figure 63, to the CFD plan views in Figure 39, equivalences were revealed. On the top surface, at the base section, the leading edge produced high velocities up to $0.69 \mathrm{mps}$. This caused pressures to decrease dramatically in Figure 40. On the opposing side, velocity values were low and pressure was high due to the blunt force of the oncoming flow. This allowed for the fluid to be easily attracted to the upper surface, as water naturally wants to move from high to low-pressure areas. This was seen in many images in the laboratory results, including Figure 66(B). As dye was injected on the suction side of the fin, it frequently slipped to the upper surface. Because the fin was angled, the leading and trailing edges compressed adjacent streamlines; as shown in Figure 39 in the CFD results. This Bernoulli's effect increased the velocity, but caused the flow to area to compress. As a result, the dye injections moved in a thin trail around these edges. Streamline separation was seen in both the CFD and laboratory results. This was due to the extreme increases in adverse pressure gradients as the fluid elements move over the top surface away from the leading edge. The separated flow allowed for rotational vortices that deposited dye within the dead zone region in Figure 63. Due to the low velocities within this region, the dye did not dissipate until several seconds after the injections stopped. This dead zone reached velocities as low as $0.05 \mathrm{mps}$ and is shown in a blue color in Figure 39 for the CFD results. Similar flow patterns were seen at the mid-section for both the CFD and lab results.

At the profile views, many flow effects paralleled in the CFD and lab images. In Figure 64 and Figure 65, the trailing flow effects moved in the vertical direction, within the dead zone. This was also seen in Figure 43, in the CFD results, showing the flow vectors pointing towards the positive Z-direction. Figure 41 also provides evidence of the streamlines 
moving from the leading edge, in the positive Z-direction, towards the trailing edge. For the mid-section profile views, in Figure 67 and Figure 68, the trailing vortices disperse in the positive and negative Z-directions. The CFD results only show the fluid moving towards the fin tip area in Figure 43. The lab images for the fin tip also slightly differ from the CFD results. The lab image and video show the dye moving over the tip and then dipping in the negative Z-direction. The CFD image shows the flow vectors only moving in the positive $\mathrm{X}$ - and Z-directions. This could be due to some error that lies within the modeling method of NX because turbulence and trailing vortices are difficult to predict and calculate.

Many common trends were found in the comparisons between the laboratory and CFD images for the SFPW position at $45^{\circ}$ angle of attack. The base and mid-section views showed similarities when focusing on the plan views. On the compression side, the flow moved at a high velocity around the leading edge. This was illustrated in Figure 45 and Figure 47 , for the CFD velocity magnitude results. Velocities reached up to $0.63 \mathrm{mps}$ around this area, which was slightly lower than the speeds at $10^{\circ}$ angle of attack. Streamlines were compressed moving over the leading edge. The video and images displayed these effects for the laboratory results in Figure 70. The CFD streamline images do not display the small swirling eddies and turbulence that occurred in the lab results, directly after detaching from the leading edge. This could be because velocity might have been higher in the water channel than calculated in the NX FLOW simulator. Because the fin was placed $45^{\circ}$ blunt to the oncoming flow, the fluid did not stay attached to the fin's surface. This position may have contributed to higher turbulence and trailing vortices than the NX program could predict. The overall path of the flow was comparable though. The flow separation, directly after the leading edge, was comparable for both data collection methods. Velocities dipped to $0.001 \mathrm{mps}$ in several regions within the dead zone. These low velocities were also seen in the video evidence.

The streamlines in Figure 73, for the CFD results, show circulation from the base in the positive Z-direction; which was also found in the profile views in Figure 71 and Figure 72. These images are comparable to the mid-section profile views in Figure 74 and Figure 75. 
For both methods, the fluid circulated behind the fin. For the CFD images, the fluid moved in the clock-wise direction near the tip and near the base of the fin. At the mid-section, the fluid flows away from the fin, causing counter-clockwise rotation as the flow enters the base and tip circulations. For the CFD images and video, it was more difficult to determine the direction of circulation. Dye seemed to move in the clockwise direction near the fin tip. 


\section{Chapter 7: Future Work}

There are several improvements that could be made to the laboratory setup. To improve the consistency of each dye injection for each angle of attack, it would be useful to set up a methodical dye injection technique. This would allow the flow of the dye to inject in the exact same position, the same volume of dye, and velocity from the injection nozzle for every experiment. The exact placement of the injector needs to be established so flow patterns can be compared properly and efficiently. This would also mean the injector would be held stable. Quantitative measurements should also be a part of this project in order to determine any changes in flow speeds around the fin and those exact locations. Due to time and funding restrictions, further improvements for both approaches were not made, but should be considered in future work.

Using the design setup and/or data from this project, further experimentation should be done. It is unknown how the designed fins in this thesis would compare to fins on today's market. It would be useful to evaluate the designed dimensions of one of these fins using the same CFD and lab approach methods. It would also be valuable to look at different fin material and material placement. There are many types of material configurations on surfboard fins used by surfers. Applying real-world conditions to the laboratory experiments would produce more consistent results to the actual performance of the surfboard fins. This would also mean looking at the surfboard and fin interaction. While it is important to look at single-fin setups to understand the purpose of each fin dimension, most surfers use three- and four-fin setups. For future work, it would be practical to compare these two setups by changing fin shapes, configurations, and materials. 


\section{Chapter 8: Summary and Conclusion}

The objective of this thesis was to find single fin shape, among the nine designed shapes, at an ideal angle of attack to optimize maneuverability and speed. By investigating a single fin setup, the results could provide a basis for further research in the field of hydrodynamics, as well as surfboard and fin design. Nine fins were designed based on nine dorsal fin shapes from aquatic species. Lift and drag forces were measured to make comparisons between the fin shapes. Laboratory and computation modeling methods were used to study a problem to find an optimum solution within the results. In the fins' design, base chord length and wingspan dimensions remained identical, however the shape of each fin's profile differed. Fluid conditions were equivalent between the CFD and lab experiments. The fins' performances were evaluated based on efficiency at ten angles of attack ( $0^{\circ}$ to $45^{\circ}$, in $5^{\circ}$ increments). Fins generating higher $\mathrm{L} / \mathrm{D}$ ratios were more efficient and could provide the surfer with greater maneuverability in typical surfing conditions.

The nine different profile shapes caused variations in the surface areas and sweep angles. While it was unknown whether the sweep angle distorted the outcome of the results, it was found that there was a correlation between surface area and force enormity. Fins with greater surface area produced greater lift and drag force values in the CFD results. Some discrepancies developed in the laboratory results, which were equated to a high margin of error in the data gathering method and variations in turbulence between cases.

For the CFD results, the SFPW yielded the highest lift and drag forces at all ten angles of attack. It had the greatest surface area of the designed fins. The Marlin had the smallest surface area of all the fins and it produced the lowest lift and drag forces for the ten incident angles. All nine fins had a peak lift force at $25^{\circ}$ angle of attack, with stall occurring at higher angles; this was true for the lift coefficients as well. The drag forces intensified as the angles of attack increased from $0^{\circ}$ to $45^{\circ}$. All nine fins had an optimum L/D peak value at $10^{\circ}$ angle of attack, but these results did not yield one fin producing the highest values at all ten angles. It was found that the SFPW had the most commonly occurring maximum $\mathrm{L} / \mathrm{D}$ ratio measurements. Out of the ten angles of attack investigated, the SFPW was the most efficient fin at four of the incident angles. At lower angles of attack, between $15^{\circ}$ and 
$20^{\circ}$, the SFPW fin had the greatest $\mathrm{L} / \mathrm{D}$ ratios. Between $0^{\circ}$ and $5^{\circ}$, the $\mathrm{BS}$ was the most efficient fin. These CFD results suggest that the SFPW or the BS would be preferred for the ease of movement on a surfboard for typical surfing conditions.

From the laboratory data, the SFPW provided the highest lift and drag values at most incident angles, due to its larger surface area. The Marlin again produced the lowest drag force values and low lift forces. Out of the ten angles, the Marlin was the least efficient fin at six angles. The SFM computed the highest $\mathrm{L} / \mathrm{D}$ ratio, for all nine fins, at $10^{\circ}$ angle of attack. The SFM, BS, and the SFPW had high L/D ratios at smaller angles of attack, which is preferable for surfing and the ease of the initial turning movement of a surfboard. The SFPW had the most commonly occurring maximum L/D ratio measurements among the nine fins. This led to the conclusion that for typical surfing conditions, the SFPW, BS, and the SFM would provide lift values for easy maneuvering and stability of the surfboard.

Both the CFD and laboratory results showed similar trends in the data, with some degree of error. The SFPW was found to be the optimum fin among the nine designed fins. The SFPW fin had the most commonly occurring high L/D ratio in both the CFD and lab results.

The SFPW fin was used for flow field visual analysis comparisons using the CFD and laboratory approaches. Although the lab images did show a higher level of disturbance in the flow field, it was concluded that the CFD and laboratory images and video provided similar flow field patterns. Symmetric flow was found around the SFPW at $0^{\circ}$ angle of attack. Repositioning the fin to $10^{\circ}$ still showed attached flow to the fin's surface, but signs of turbulence were evident in the lab images. Flow separation was illustrated for both methods at the incident angle of $25^{\circ}$. Visual velocity differences were comparable in both methods, but turbulent disturbances and high volumes of vortices were seen in the lab results. This was especially true in the profile views for both $25^{\circ}$ and $45^{\circ}$. Similarities were established at $45^{\circ}$.

The lab results were not as consistent as the CFD results. These differences could have been due to the NX program under-calculating the turbulence within the fluid domain. All modeling methods are not perfect, so it was difficult to determine the source of error in the 
CFD results. Unexpected turbulence could have also occurred in the water channel. This could be a product of high surface roughness from the flume walls or turbulence could have been created from the upstream inlet section setup.

While there are very few CFD and experimental scientific studies available, the possibilities for research in the field of surfing are infinite. The objective of this thesis was to provide a basis for future fin design by using CFD modeling and laboratory methods. The use of these engineering tools is becoming necessary in the development process of high intensity sports, such as surfing. By comparing these two data gathering approaches, the results can be compared and sources of error can be identified for improvement in future experiments. With the use of the CFD and lab methods, nine designed single fin setups were assessed and one optimum fin was found. The criterion was a fin with peak lift forces and low drag forces at the ten angles of attack. The SFPW was found to be the optimum fin as it generated high L/D ratios for the improvement of efficiency and ease of maneuverability for the surfer. Although there are many variables on the wave that can affect the surfers' ability to maneuver the face of the wave, there is value in continuously progressing in the understanding and development of surfing. 


\section{Chapter 9: References}

Abercrombie, D. L., Chapman, D. D., Gulak, S. J., \& Carlson, J. K. (2013). Visual Identification of Fins from Common Elasmobranchs in the Northwest Atlantic Ocean. Panama City, FL:

National Marine Fisheries Service-Southeastern Fisheries Science Center.

Akzo Nobel Coatings Inc. (2008). Sikkens Colorbuild Plus. Retrieved August 19, 2015, from C\&D Auto Paint: http://www.cdautopaint.com/wpcontent/uploads/2012/09/colorbuild_plus.pdf

Anders, M. (2014, September 23). Birth of a Fin. Retrieved from Surfline: http://www.surfline.com/surf-news/birth-of-a-fin--from-a-sketch-to-the-surf_118202/

Anderson, B. (2008). Physics of Sailing. American Institute of Physics , 38-43.

Anderson, J. D. (2005). Introduction to Flight (5th ed.). New York, NY, U.S.A.: McGraw-Hill.

Apple. (2015). iPhone 6. USA. Retrieved from https://www.apple.com/iphone-6/cameras/

Bannister, J. L., Kemper, C. M., \& Warneke, R. M. (1996). The Action Plan for Australian Cetaceans. Australian Nature Conservation Agency, The Director of National Parks and Wildlife. Canberra, Australia: Australian Nature Conservation Agency.

Barlock, R. (2013). Stilling Basin Scour Remediation Using Air Injection and Flat Plate Extension. Houghton, MI: Michigan Technological University.

Beggs, R. (2009). Surfboard Hydrodynamics. Canberra, Australia: The University of New South Wales at the Australian Defence Force Academy.

Brandner, P. A., \& Walker, G. J. (2004). Hydrodynamic Performance of a Surfboard Fin. 15th Australasian Fluid Mechanics Conference. Sydney: Australian Meritime College and University of Tasmania.

Butt, T. (2008). Surf Science: An Introduction to Waves for Surfing. Honolulu: University of Hawaii Press.

Carswell, D., \& Lavery, N. (2006). 3D fin model construction from 2D shapes using nonuniform rational B-spline surfaces. Advances in Engineering Software , 37, 491-501.

Cater, G. (2013, June). \#363 Barry Bennett- Lightning Bolt Pintail. National Library of Australia . Canberra, Australia. Retrieved from http://www.surfresearch.com.au/00000363.html

Champagne, T. (2011). Physical modeling of air injection as a scour remediation technique near gated weir stilling basins. Michigan Technological University, Department of Civil and Environmental Engineering, Houghton, MI. 
Cooper, P. (N/A). Blue Shark. Retrieved from Ichthyology at the Florida Museum of Natural History: http://www.flmnh.ufl.edu/fish/Gallery/Descript/blueshark/blueshark.html

COSEWIC. (2002). COSEWIC Assessment and Update Status Report on the Blue Whale. Ottawa, ON: Committee on the Status of Endangered Wildlife in Canada.

Dassault Systemes. (2015). Products: Solidworks. Retrieved January 13, 2015, from Dassault Systemes Solidworks: http://www.solidworks.com/sw/3d-cad-design-software.htm

Davidson, L. (2015). An Introduction to Turbulence Models. Chalmers University of Technology, Department of Thermo and Fluid Dynamics, Goteborg, Sweden.

de Azevedo e Silva, C. E., Azeredo, A., Lailson-Brito, J., Machado Torres, J. P., \& Malm, O. (2007). Polychlorinated biphenyls and DDT in swordfish (Xiphias gladius) and blue shark (Prionace glauca) from Brazilian coast. Chemosphere, S48-S53.

Denny, M. W. (1993). Air and Water. Princeton, New Jersey: Princeton University Press.

Department of Fisheries and Aquaculture. (N/A). Sharks- Greenland (Somniosus microcephalus), Shortfin Mako (Isurus oxyrinchus), Blue Shark (Prionace glauca), Basking Shark (Cetorhinus maximus), and Porbeagle (Lamna nasus). St. John's, NL: Government of Newfoundland and Labrador.

Department of the Environment. (2015). Globicephala macrorhynchus in Species Profile and Threats Database. Retrieved from Australian Government: Department of the Environment: http://www.environment.gov.au/cgi-bin/sprat/public/publicspecies.pl?taxon_id=62

Department of the Environment. (2015). Orcinus orca in Species Profile and Threats Database. Retrieved from Department of the Environment, Canberra:

http://www.environment.gov.au/cgi-bin/sprat/public/publicspecies.pl?taxon_id=46

Doll, P.

Edge, R. (2001). Surf Physics. Department of Physics and Astronomy. Columbia, South Carolina: University of South Carolina.

Executive Office of EEA. (2015). Blue Shark. Retrieved March 19, 2015, from Energy and Environmental Affairs: www.mass.gov/eea/agencies/dfg/dmf/recreational-fishing/blueshark.html

FAO Fisheries. (1985). FAO SPECIES CATALOGUE. Kyoto, Japan: FAO Fisheries.

Fish, F. E., Weber, P. W., Murray, M. M., \& Howle, L. E. (2011). Marine Applications of the Biomimetic Humpback Whale Flipper. Marine Technology Society Journal , 45 (4), 198-206. 
Gardieff, S. (N/A). Atlantic Blue Marlin. Retrieved January 20, 2015, from Ichthyology at Florida Museum of Natural History:

http://www.flmnh.ufl.edu/fish/gallery/descript/bluemarlin/bluemarlin.html

Garlotta, D. (2001). A Literature Review of Poly(Lactic Acid). Journal of Polymers and the Environment, 9 (2), 63-84.

George, S. (2014, September 22). From Keels to Quads. Retrieved from Surfline: http://www.surfline.com/surf-news/fin-week-from-keels-to-quads_117944/

Grace, M. A. (2001). Field guide to requiem sharks (Elasmobranchiomorphi: Carcharhinidae) of the Western North Atlantic. U.S. Department of Commerce.

Gudimetla, P., Kelson, N., \& El-Atm, B. (2009). Analysis of the hydrodynamic performance of three-and four-fin surfboards using computational fluid dynamics. Australian Journal of Mechanical Engineering , 7 (1), 61-66.

Guinness World Records News. (2012, May 09). 78-foot wave surfed by Garrett McNamara confirmed as largest ever ridden. Retrieved April 28, 2015, from Guinness World Records: http://www.guinnessworldrecords.com/news/2012/5/video-78-foot-wave-surfed-bygarrett-mcnamara-confirmed-as-largest-ever-ridden-41598/

Hendricks, T. (1969, July). Surfboard Hydrodynamics, Part IV: Speed. Surfer Magazine , 10 (3).

Hendricks, T. (1969, January). Surfboard Hydrodynamics: Part I: Drag. Surfer Magazine , 9 (6).

Hoerner, S. F. (1985). Fluid- Dynamic Lift. (H. V. Borst, Ed.) Hoerner, Liselotte A.

IUCN/SSC Cetacean Specialist Group. (2003). Dolphins, Whales and Porpoises: 2002-2010 Conservation Action Plan for the World's Cetaceans. IUCN - The World Conservation Union, IUCN/SSC Cetacean Specialist Group. Gland, Switzerland and Cambridge, UK: IUCN.

Jefferson, T. A., Leatherwood, S., \& Webber, M. A. (1993). Marine Mammals of the World. In FAO, FAO Species Identification Guide (pp. 182-183). Rome.

Jones, I. M. (2006). A Northeast Pacific Offshore Killer Whale (Orcinus orca) Feeding on a Pacific Halibut (Hippoglossus stenolepis). Marine Mammal Science , 22(1), 198-200.

Kabasakal, H., \& Kabasakal, Ö. (2013). First Record of a Shortfin Mako Shark, Isurus oxyrinchus Rafinesque, 1810 (Chondrichthyes:Lamnidae) from the Bay of Saroz (Ne Aegean Sea). Annales, Series Historia Naturalis , 27-32.

Kinze, C. C. (2003). Marine Mammals. Princeton Field Guides. 
Kiss, A. (2008). Marine Science 1A Field School Notes Jervis Bay 2008. UNSW Australia, School of Physical, Environmental and Mathematical Sciences. Canberra, Australia: UNSW Australia.

Kundu, P. K., \& Cohen, I. M. (2008). Fluid Mechanics (4 ed.). Burlington, MA, USA: Elsevier.

Lavery, N., Foster, G., Carswell, D., \& Brown, S. (2009). CFD modelling of the effect of fillets on fin drag. Reef Journal , 1 (1), pp. 93-111.

Lesieur, M. (2008). Turbulence in Fluids (4th ed., Vol. 84). (R. Moreau, Ed.) Dorderecht, The Netherlands: Springer.

Martin, A. R. (2003). Biology of Sharks and Rays. Retrieved 2014, from ReefQuest Centre for Shark Research: www.elasmo-research.org/copyright.htm

Mottet, C. O. (N/A). Pantropical Spotted Dolphin. Retrieved January 13, 2015, from Texas A\&M University Corpus Christi:

http://www.sci.tamucc.edu/tmmsn/29Species/pantropicalspotteddolphin.html

NASA. (2014, June 12). Beginner's Guide to Aerodynamics. Glenn Research Center . (T. Benson, Ed.) Cleveland, OH, USA. Retrieved January 9, 2015, from http://www.grc.nasa.gov/WWW/k-12/airplane/bga.html

NOAA. Blue Marlin. Blue Marlin. NOAA Fish Watch.

NOAA. (2010). Final Recovery Plan for the Fin Whale (Balaenoptera physalus). National Marine Fisheries Service, Office of Protected Resources. Silver Spring, Maryland: National Oceanic and Atmospheric Administration.

NOAA Fish Watch. (2015). Pacific and North Atlantic Swordfish. Retrieved from Fish Watch: http://www.fishwatch.gov/seafood_profiles/species/swordfish/group_pages/index.htm

NOAA Fisheries. (2012, December 12). Dall's Porpoise (Phocoenoides dalli). Retrieved January 21, 2015, from NOAA Fisheries: Office of Protected Resources: http://www.nmfs.noaa.gov/pr/species/mammals/cetaceans/dallsporpoise.htm

NOAA Fisheries. (2013, September 4). Fin Whale. Retrieved January 13, 2015, from NOAA Fisheries: Office of Protected Resources:

http://www.nmfs.noaa.gov/pr/species/mammals/cetaceans/finwhale.htm

NOAA Fisheries. (2014, June 25). Killer Whales (Orcinus orca). Retrieved from NOAA Fisheries: Office of Protected Resources:

http://www.nmfs.noaa.gov/pr/species/mammals/cetaceans/killerwhale.htm

NOAA Fisheries. (2012, December 12). Short-finned Pilot Whale. Retrieved from NOAA fisheries: Office of Protected Resources:

http://www.nmfs.noaa.gov/pr/species/mammals/cetaceans/pilotwhale_shortfinned.htm 
NOAA. (2013, February 12). Ocean Waves. Retrieved May 11, 2015, from NOAA Ocean Explorer Webmaster:

http://oceanexplorer.noaa.gov/edu/learning/9_ocean_waves/ocean_waves.html

NOAA. (1998). Recovery Plan for the Blue Whale (Balaenoptera Musculus). National Marine Fisheries Service, Office of Protected Resources. Silver Spring, Maryland: National Oceanic and Atmospheric Administration.

NOAA. Shortfin Mako Shark. Shortfin Mako Shark. NOAA Fish Watch.

NOAA. Swordfish. Swordfish. NOAA Fish Watch.

Noble, C., Cannon-Jones, H. A., Damsgard, B., Flood, M. J., Midline, K. O., Roque, A., et al. (2011). Injuries and deformities in fish: their potential impacts upon aquacultural production and welfare. Fish Physiology and Biochemistry, 38 (1), 61-83.

Nortek. (2015, March 20). Products. Retrieved from http://www.nortekusa.com/usa/products

Norwegian University of Science and Technology. (N/A). General Modelling and Scaling Laws. Trondheim, Norway.

NSRL. (N/A). Short-finned Pilot Whale. Retrieved January 13, 2015, from Natural Science Research Laboratory: http://www.nsrl.ttu.edu/tmot1/globmacr.htm

Paine, M. (1974). Hydrodynamics of Surfboards. Department of Mechanical Engineering. Sydney, Australia: University of Sydney.

Pandiripalli, B. (2007). Repeatability and Reproducibility Studies: A Comparison of Techniques. Wichita State University, Industrial and Manufacturing Engineering. Wichita: Wichita State University.

Parsons, G. R. (2006). Sharks, Skates, and Rays of the Gulf of Mexico: A Field Guide. (1, Ed.) Jackson, Mississippi, USA: University Press of Mississippi.

Passarelli, N., Knickle, C., \& DiVittorio, K. (N/A). Shortfin Mako. Retrieved January 20, 2015, from Ichthyology at the Florida Museum of Natural History:

http://www.flmnh.ufl.edu/fish/gallery/descript/shortfinmako/shortfinmako.html

Pitman, R. L., Perryman, W. L., LeRoi, D., \& Eilers, E. (2007). A Dwarf Form of Killer Whale in Antarctica. Journal of Mammalogy , 88(1), 43-48.

Rumsey, C. (2014, December 18). Turbulence Modeling Resource: 2D NACA 0012 Airfoil

Validation Case. Retrieved January 10, 2015, from NASA Langley Research Center:

http://turbmodels.larc.nasa.gov/naca0012_val.html

Sadraey, M. (2009). Aircraft Performance Analysis. Saarbrucken, Germany: VDM Publishing. 
Sakamoto, H., \& Yanamoto, Y. (2007). Computer Integrated Design of Surfboard-Fins to Improve Turning Ability with Drag Force Reduction. ASME 2007 International Design Engineering Technical Conferences \& Computers and Information in Engineering Conference (pp. 913-918). Las Vegas, Nevada: ASME.

Siemens PLM Software Inc. (2015). About NX Software. Retrieved January 13, 2015, from Siemens: http://www.plm.automation.siemens.com/en_us/products/nx/about-nxsoftware.shtml

Standen, E. M., \& Lauder, G. V. (2007). Hydrodynamic function of dorsal and anal fins in brook trout (Salvelinus fontinalis). The Journal of Experimental Biology , 210 (2), 325-339.

SURFER Magazine. (N/A). Gerry Lopez. San Clemente, California, USA. Retrieved from http://encyclopediaofsurfing.com/entries/lopez-gerry

Surfing Heritage Foundation. (N/A). The History of Surfing. Retrieved from Surfing Heritage \& Culture Center: http://files.surfingheritage.org/flash/timeline/

Tokiwa, Y., Calabia, B. P., Ugwu, C. U., \& Aiba, S. (2009). Biodegradability of Plastics. International Journal of Molecular Science , 3722-3742.

Tritton, D. J. (1988). Physical Fluid Dynamics (2 ed.). New York, New York, United States: Oxford University Press.

U.S. Department of Transportation, Federal Aviation Administration, Flight Standards Service. (2012). Helicopter Flying Handbook. Oklahoma City, OK, USA: U.S. Department of Transportation, Federal Aviation Administration, Airman Testing Standards Branch.

UIUC Applied Aerodynamics Group. (2015). UIUC Airfoil Coordinates Database. Department of Aerospace Engineering at University of ILlinois . Urbana-Champaign, Illinois, USA.

Ultimaker. (2015). PLA. Retrieved from Ultimaker: https://shop.ultimaker.com/product/25/PLABlue

United States Department of Transportation, Federal Aviation Administration. (2008). Pilot's Handbook of Aeronautical Knowledge. Oklahoma City, OK, USA: U.S. Department of Transportation, Federal Aviation Administration, Airman Testing Standards Branch.

Wahls, R. A. (1990). Development of a Defect Stream Function, Law of the Wall/Wake Methods for Compressible Turbulent Boundary Layers. Raleigh, North Carolina: National Aeronautics and Space Administration.

Warsi, Z. (1993). Fluid Dynamics: Theoretical and Computational Approaches. Boca Raton, Florida, United States: CRC Press, Inc.

WDC. (N/A). Pantropical Spotted Dolphin. Retrieved January 13, 2015, from WDC: http://us.whales.org/species-guide/pantropical-spotted-dolphin 
Wild Whales. (N/A). Blue Whale (Balaenoptera musculus). Retrieved from Wild Whales b.c. cetacean sightings network: http://wildwhales.org/blue-fin-and-sei-whale/

Zanoun, E., Durst, F., \& Nagib, H. (2003). Evaluating the law of the wall in two-dimensional fully developed turbulent channel flows. Physics of Fluids , 15 (10), 3079-3089. 
Appendix A: Methods for Experimental Setup 


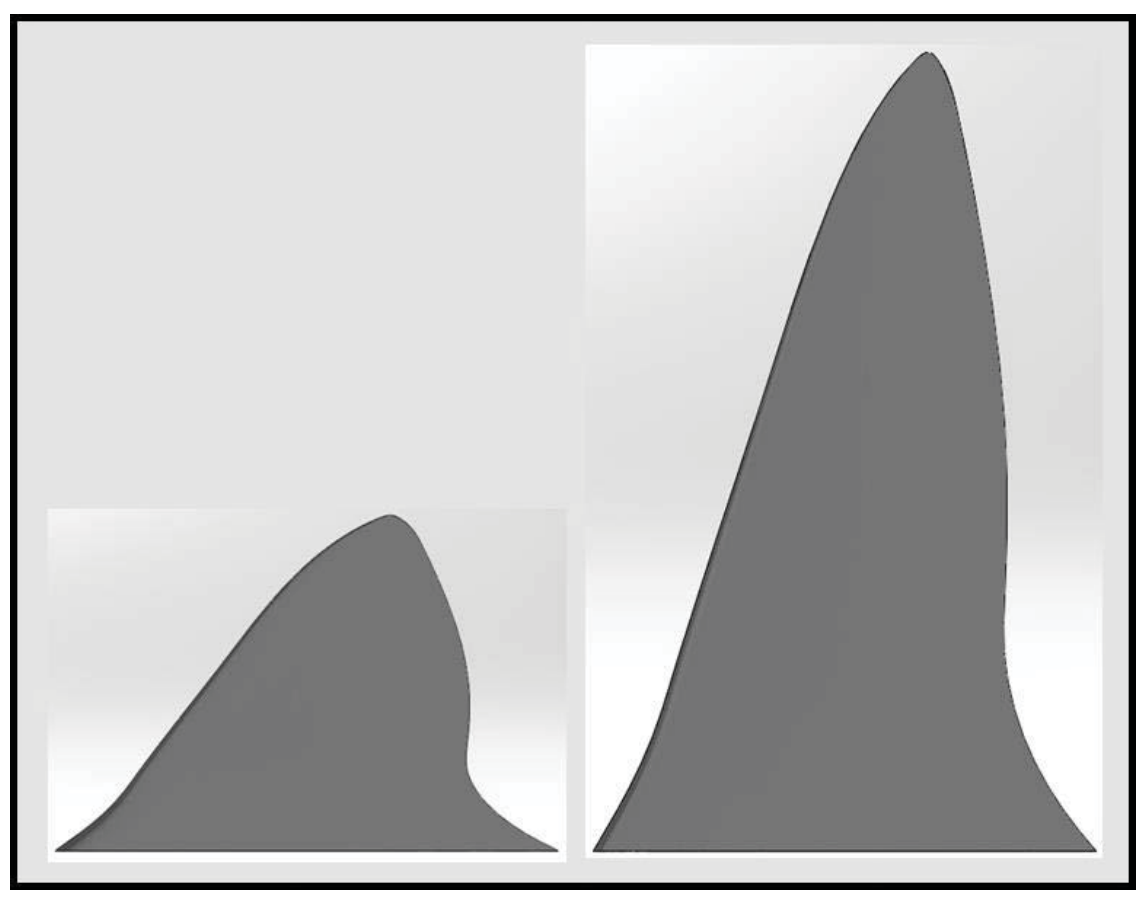

Figure 91: Blue Shark profile view, [left] original design $L_{w}=112.0 \mathrm{~mm} L_{h}=74.81 \mathrm{~mm}$, [right] full-scaled design $L_{w}=112.0 \mathrm{~mm} L_{h}=177.8 \mathrm{~mm}$

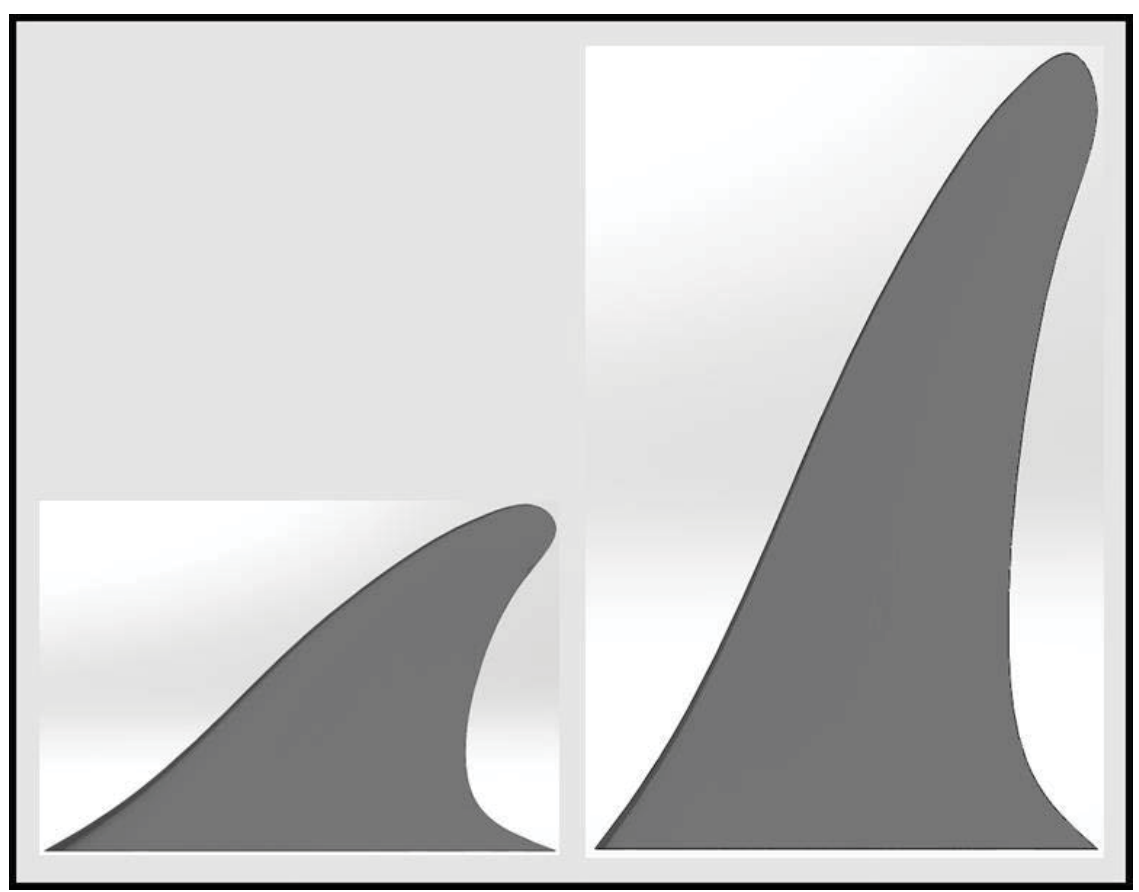

Figure 92: Fin Whale profile view, [left] original design $L w=112.0 \mathrm{~mm} \mathrm{Lh}=76.15 \mathrm{~mm}$, [right] full-scaled design $L w=112.0 \mathrm{~mm} L h=177.8 \mathrm{~mm}$ 


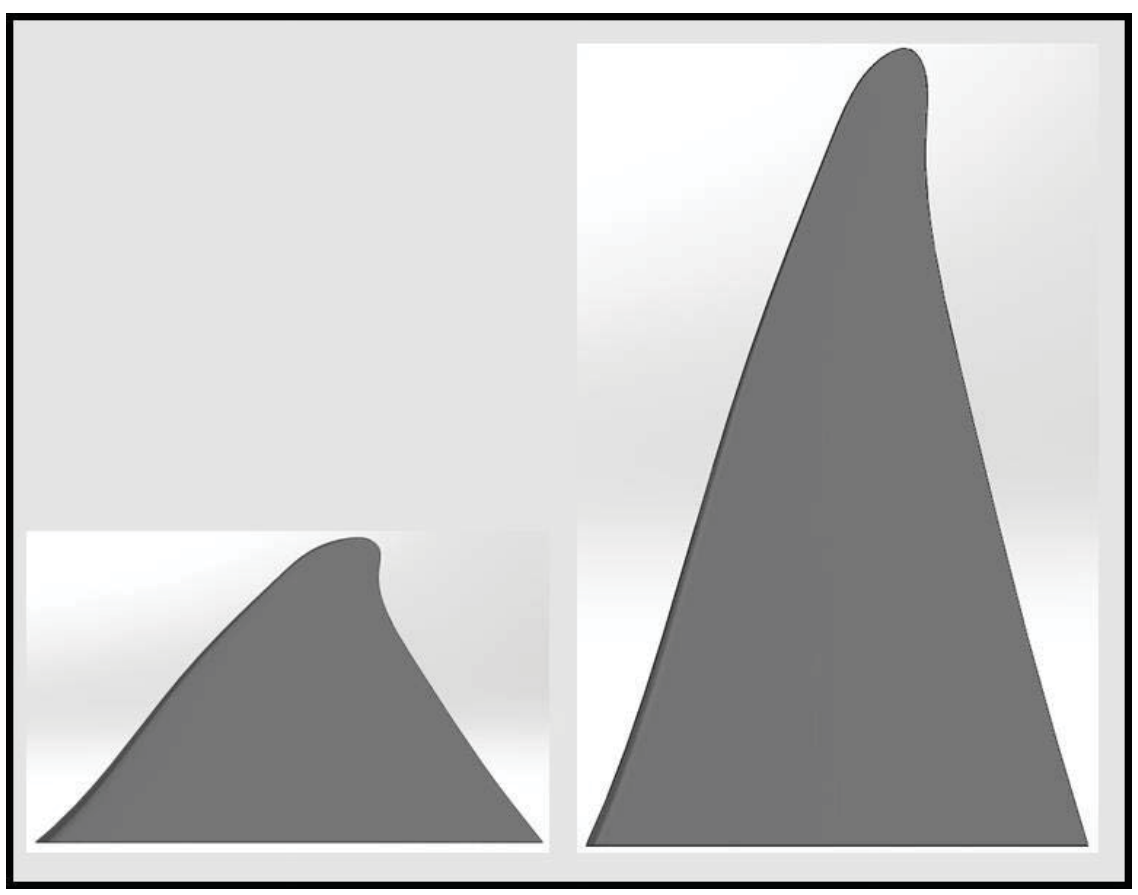

Figure 93: Dall's Porpoise profile view, [left] original design $\mathrm{Lw}=112.0 \mathrm{~mm} \mathrm{Lh}=52.39 \mathrm{~mm}$, [right] full-scaled design $L w=112.0 \mathrm{~mm} L h=177.8 \mathrm{~mm}$

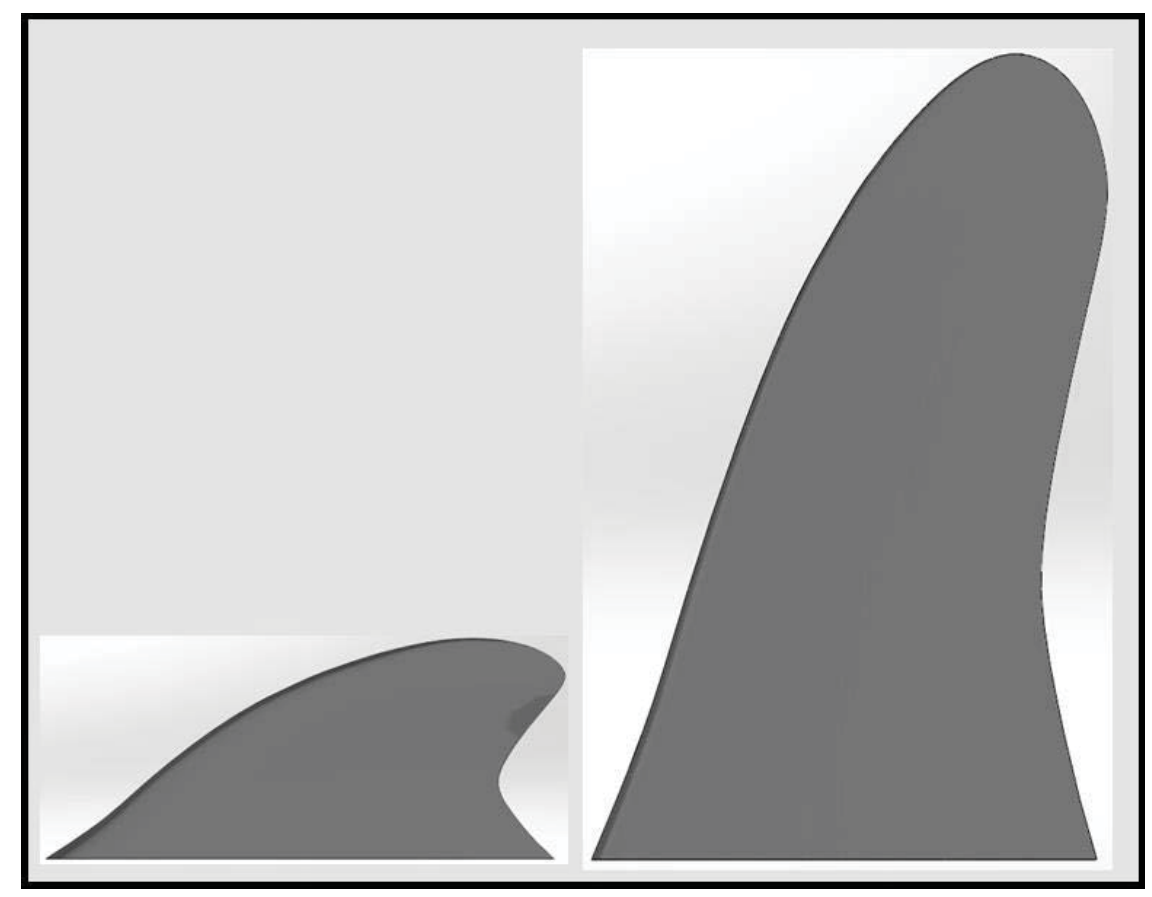

Figure 94: Short-finned Pilot Whale profile view, [left] original design $\mathrm{Lw}=112.0 \mathrm{~mm} \mathrm{Lh}=48.20 \mathrm{~mm}$, [right] full-scaled design $\mathrm{Lw}=112.0 \mathrm{~mm} \mathrm{Lh}=177.8 \mathrm{~mm}$ 


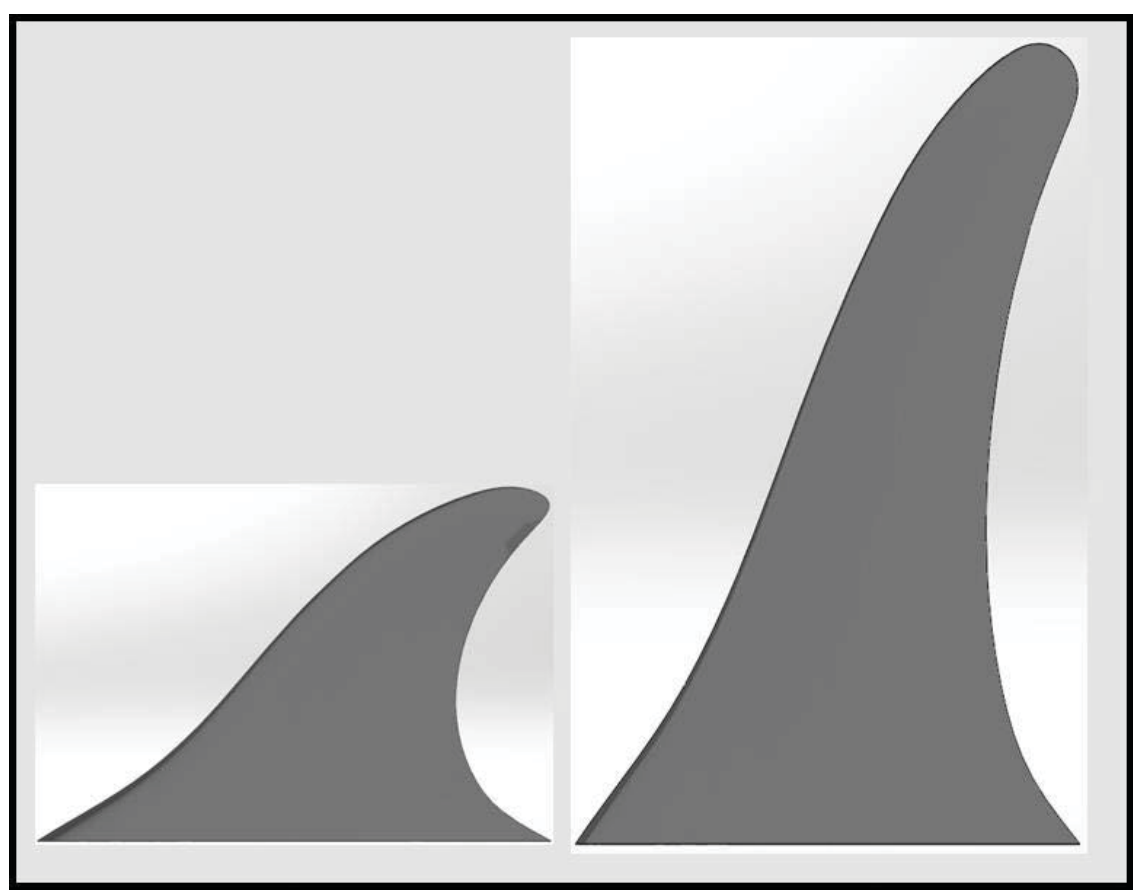

Figure 95: Pacific Spotted Dolphin profile view, [left] original design $\mathrm{Lw}=112.0 \mathrm{~mm} \mathrm{Lh}=77.44 \mathrm{~mm}$, [right] full-scaled design $L w=112.0 \mathrm{~mm} L h=177.8 \mathrm{~mm}$

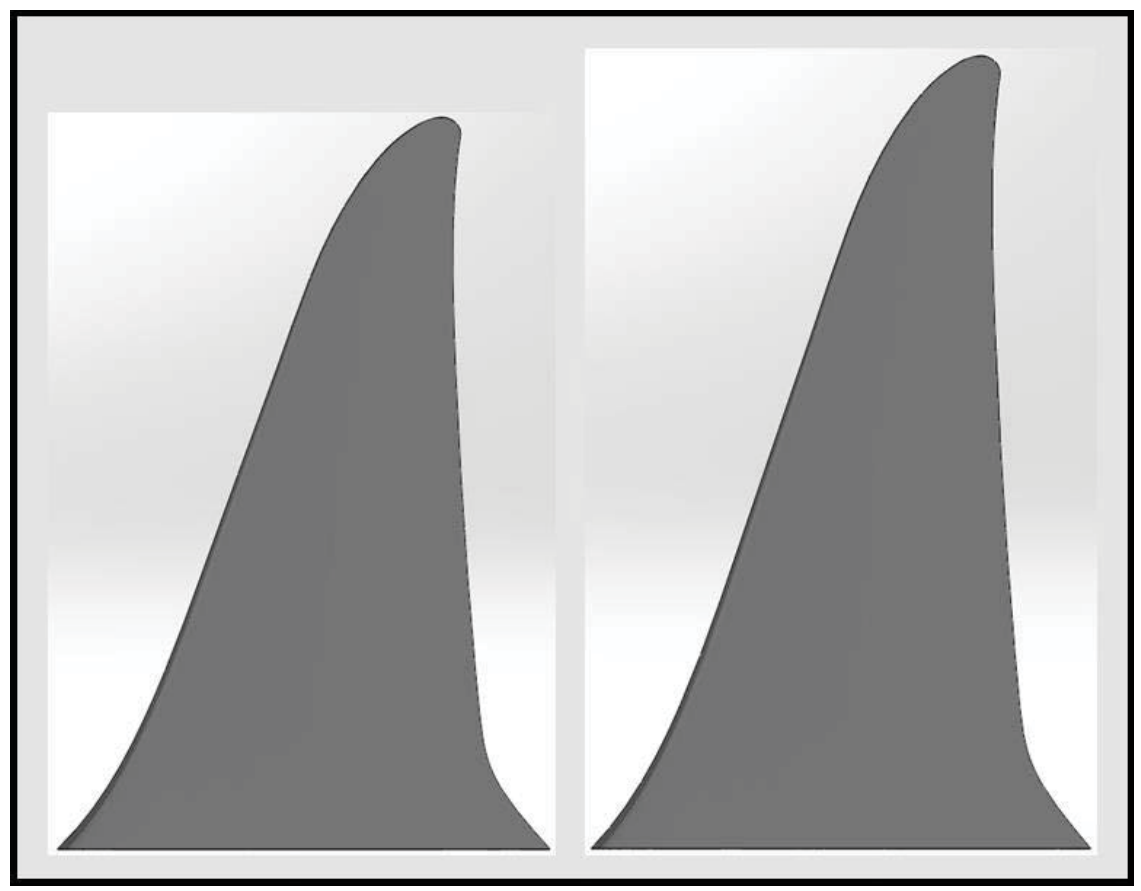

Figure 96: Killer Whale (Orca) profile view, [left] original design $\mathrm{Lw}=112.0 \mathrm{~mm} \mathrm{Lh}=166.11 \mathrm{~mm}$, [right] full-scaled design $L w=112.0 \mathrm{~mm} L h=177.8 \mathrm{~mm}$ 


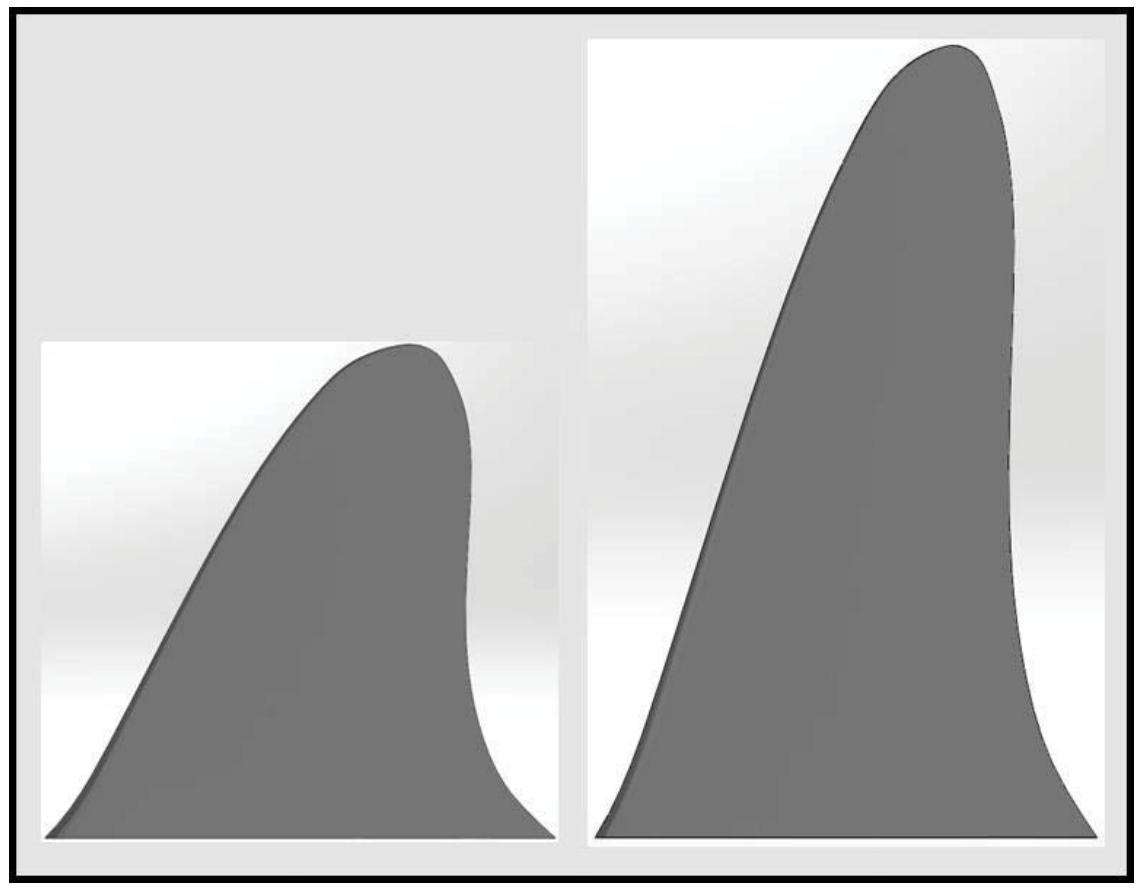

Figure 97: Shortfin Mako Shark profile view, [left] original design $\mathrm{Lw}=112.0 \mathrm{~mm} \mathrm{Lh}=108.81 \mathrm{~mm}$, [right] full-scaled design $L w=112.0 \mathrm{~mm} L h=177.8 \mathrm{~mm}$

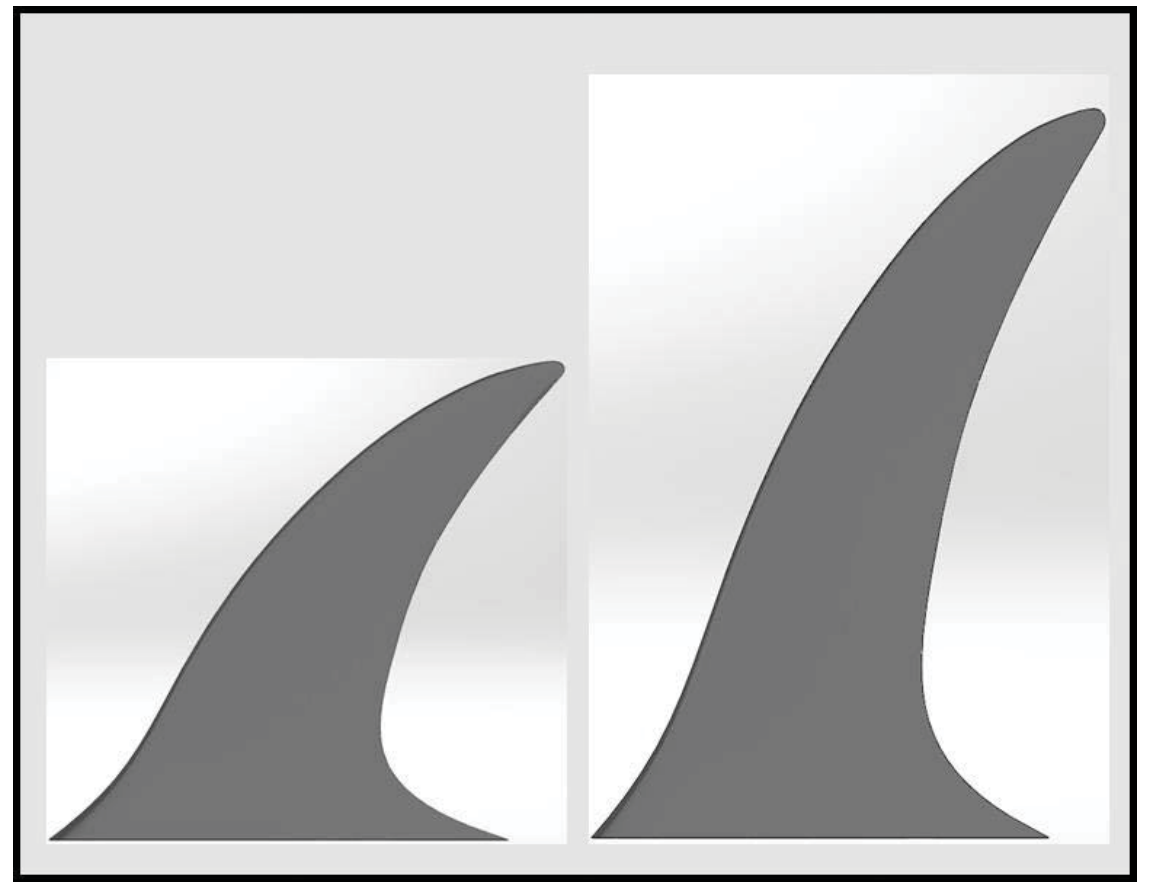

Figure 98: Swordfish profile view, [left] original design $L w=112.0 \mathrm{~mm} \mathrm{Lh}=116.38 \mathrm{~mm}$, [right] full-scaled design $L w=112.0 \mathrm{~mm} L h=177.8 \mathrm{~mm}$ 


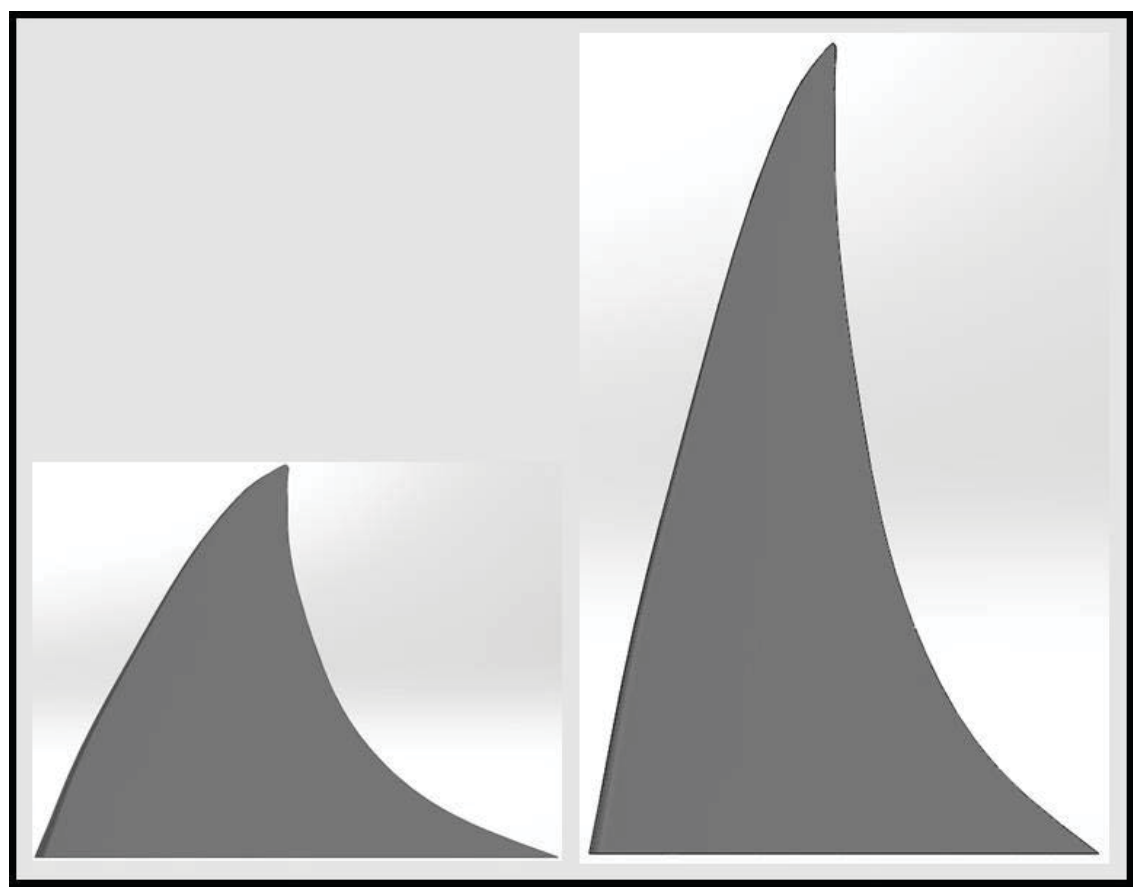

Figure 99: Blue Marlin profile view, [left] original design $L_{w}=112.0 \mathrm{~mm} L_{h}=83.68 \mathrm{~mm}$, [right] full-scaled design $L_{w}=112.0 \mathrm{~mm} L_{h}=177.8 \mathrm{~mm}$ 


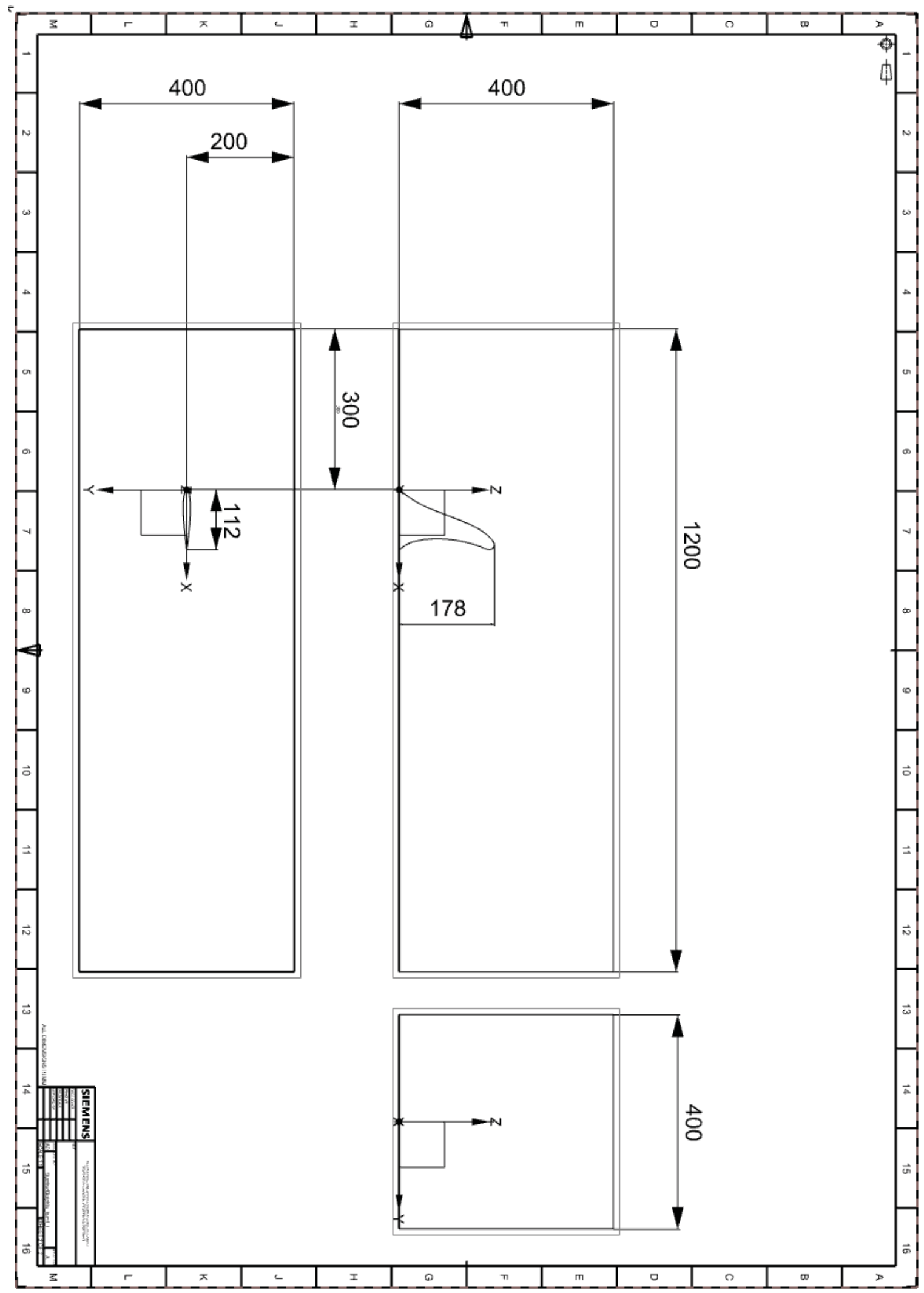

Figure 100: Orthographic drawings of the fluid boundary box and fin positioning 184 
Table 15: Refinement meshing test results using Orca fin at $\alpha=0^{\circ}, \mathrm{U}_{\infty}=10 \mathrm{mps}$

\begin{tabular}{|ccccccr|}
\hline Lift (N) & Drag (N) & $C_{L}$ & $C_{D}$ & $\begin{array}{c}C_{L} \% \\
\text { difference }\end{array}$ & $\begin{array}{c}\mathbf{C}_{D} \% \\
\text { difference }\end{array}$ & $\begin{array}{c}\text { Element } \\
\text { Size (mm) }\end{array}$ \\
\hline 82.1 & 0.1 & 0.17 & 0.04 & 119.32 & 120.89 & 72 \\
333.1 & 0.3 & 0.70 & 0.16 & 1.10 & 0.46 & 7.2 \\
331.6 & 0.3 & 0.70 & 0.16 & 0.48 & 1.03 & 6.6 \\
335.0 & 0.3 & 0.71 & 0.16 & 1.15 & 0.95 & 6 \\
338.2 & 0.3 & 0.71 & 0.16 & 0.10 & 0.05 & 5.4 \\
338.0 & 0.3 & 0.71 & 0.16 & & & 5.28 \\
\hline
\end{tabular}

Table 16: Repeatability test results using SFPW fin at $\alpha=15^{\circ}, \mathrm{U}_{\infty}=0.5 \mathrm{mps}$

\begin{tabular}{|c|c|c|c|c|c|c|}
\hline & \multicolumn{3}{|c|}{ Lift (N) } & \multicolumn{3}{|c|}{ Drag (N) } \\
\hline & $\min$ & $\max$ & avg & $\min$ & $\max$ & avg \\
\hline & 0.68 & 0.72 & 0.7 & 0.6 & 0.68 & 0.64 \\
\hline & 0.58 & 0.68 & 0.63 & 0.64 & 0.71 & 0.675 \\
\hline & 0.68 & 0.74 & 0.71 & 0.62 & 0.72 & 0.67 \\
\hline & 0.62 & 0.7 & 0.66 & 0.6 & 0.7 & 0.65 \\
\hline & 0.64 & 0.76 & 0.7 & 0.64 & 0.68 & 0.66 \\
\hline & 0.54 & 0.69 & 0.615 & 0.62 & 0.67 & 0.645 \\
\hline & 0.58 & 0.68 & 0.63 & 0.64 & 0.72 & 0.680 \\
\hline & 0.64 & 0.74 & 0.69 & 0.63 & 0.71 & 0.67 \\
\hline & 0.68 & 0.74 & 0.71 & 0.6 & 0.67 & 0.635 \\
\hline & 0.57 & 0.71 & 0.64 & 0.56 & 0.7 & 0.63 \\
\hline & 0.64 & 0.74 & 0.69 & 0.6 & 0.68 & 0.64 \\
\hline & 0.52 & 0.76 & 0.64 & 0.56 & 0.68 & 0.62 \\
\hline & 0.58 & 0.66 & 0.62 & 0.62 & 0.68 & 0.65 \\
\hline & 0.62 & 0.68 & 0.65 & 0.6 & 0.72 & 0.66 \\
\hline & 0.66 & 0.71 & 0.685 & 0.56 & 0.68 & 0.62 \\
\hline & 0.52 & 0.72 & 0.62 & 0.54 & 0.72 & 0.63 \\
\hline & 0.62 & 0.73 & 0.675 & 0.6 & 0.67 & 0.635 \\
\hline & 0.54 & 0.71 & 0.625 & 0.56 & 0.68 & 0.62 \\
\hline & 0.64 & 0.74 & 0.69 & 0.61 & 0.7 & 0.655 \\
\hline & 0.64 & 0.68 & 0.66 & 0.64 & 0.72 & 0.680 \\
\hline Average & 0.61 & 0.71 & 0.66 & 0.60 & 0.69 & 0.21 \\
\hline Minimum & 0.52 & 0.66 & 0.62 & 0.54 & 0.23 & 0.18 \\
\hline Maximum & 0.68 & 0.76 & 0.71 & 0.64 & 0.28 & 0.24 \\
\hline \% Difference & 26.7 & 14.1 & 14.3 & 16.9 & 19.6 & 28.6 \\
\hline
\end{tabular}


Appendix B: Results for remaining nine fins 


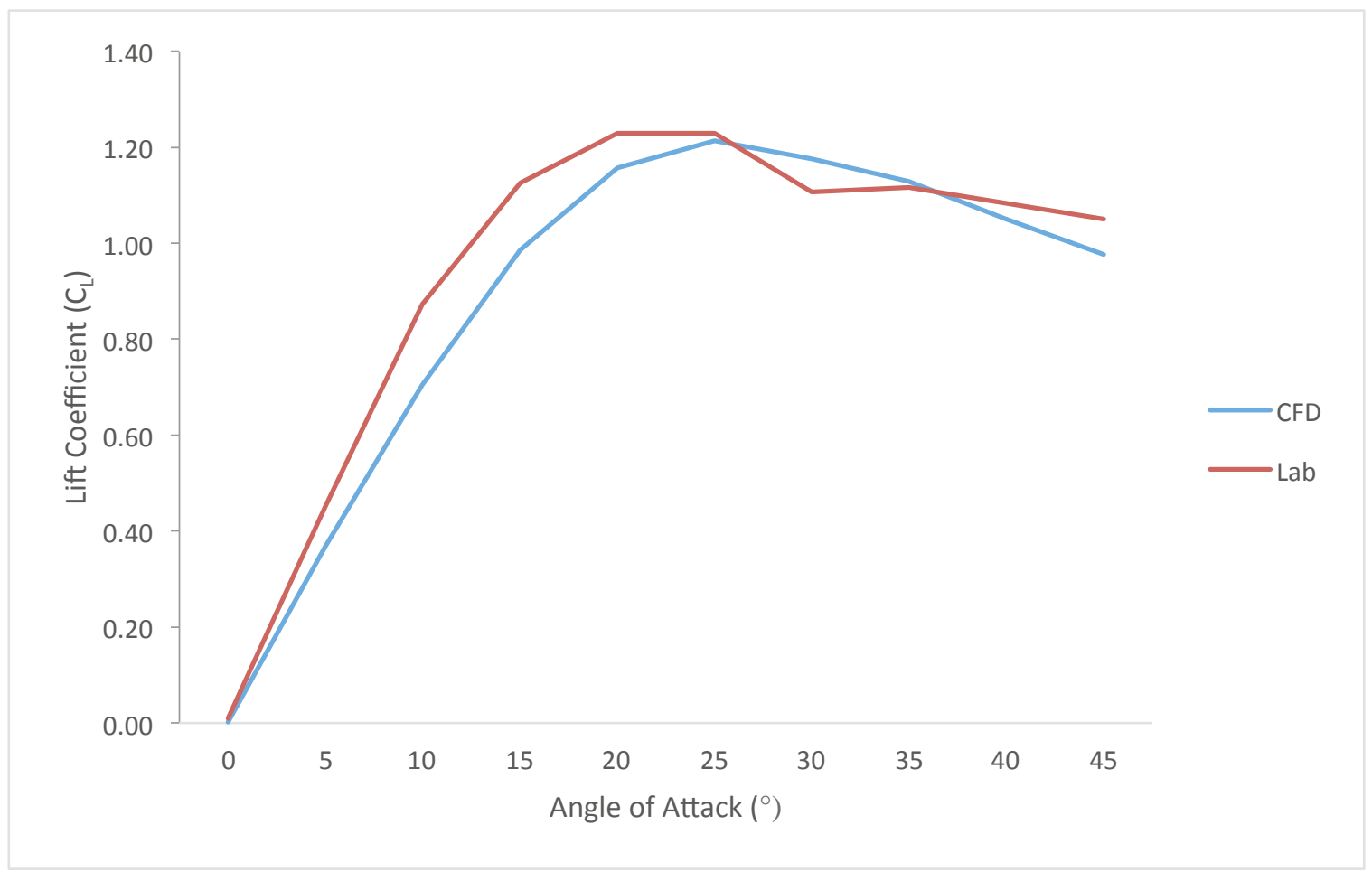

Figure 101: Coefficient of lift from laboratory and CFD data collected for the Blue Shark at ten angles of attack $\left(0-45^{\circ}\right)$

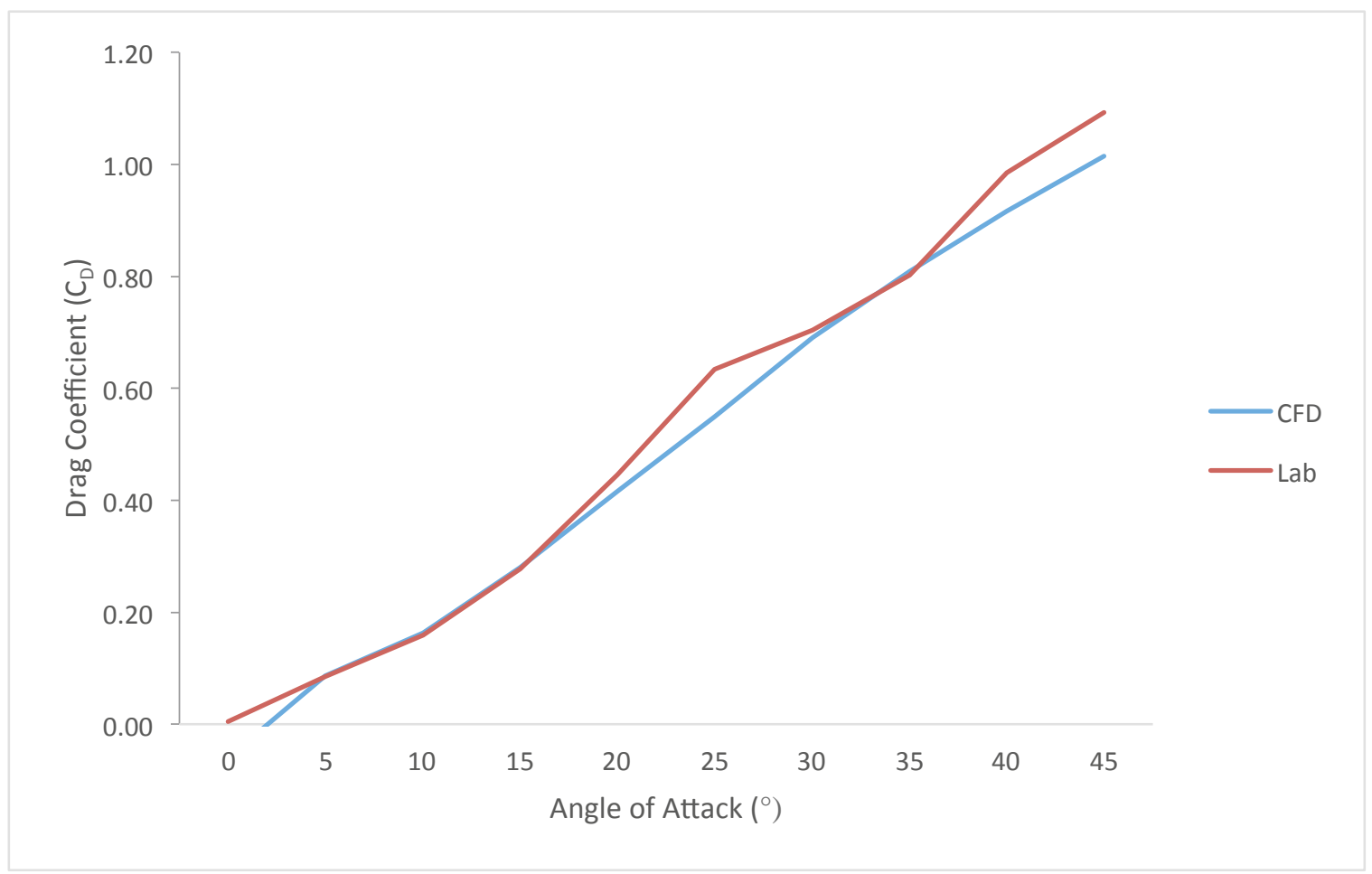

Figure 102: Coefficient of drag from laboratory and CFD data collected for the Blue Shark at ten angles of attack $\left(0-45^{\circ}\right)$ 
Table 17: CFD lift and drag data for the Blue Shark $\left(0-45^{\circ}\right)$

\begin{tabular}{|c|ccccccc|}
\hline $\boldsymbol{\alpha}\left({ }^{\circ}\right)$ & Lift (N) & Drag (N) & $\mathbf{U}(\mathbf{m p s})$ & $\mathbf{A}\left(\mathbf{m}^{2}\right)$ & $\mathbf{C}_{\mathrm{L}}$ & $\mathbf{C}_{\mathbf{D}}$ & $\mathbf{C}_{\mathrm{L}} / \mathbf{C}_{\mathbf{D}}$ \\
\hline 0 & 0.00 & 0.07 & 0.5 & 0.0100 & 0.00 & 0.06 & 0.02 \\
5 & 0.46 & 0.11 & 0.5 & 0.0100 & 0.37 & 0.09 & 4.27 \\
10 & 0.88 & 0.20 & 0.5 & 0.0100 & 0.71 & 0.16 & 4.33 \\
15 & 1.24 & 0.35 & 0.5 & 0.0100 & 0.99 & 0.28 & 3.51 \\
20 & 1.45 & 0.52 & 0.5 & 0.0100 & 1.16 & 0.42 & 2.78 \\
25 & 1.52 & 0.69 & 0.5 & 0.0100 & 1.22 & 0.55 & 2.21 \\
30 & 1.47 & 0.86 & 0.5 & 0.0100 & 1.18 & 0.69 & 1.70 \\
35 & 1.41 & 1.01 & 0.5 & 0.0100 & 1.13 & 0.81 & 1.39 \\
40 & 1.32 & 1.15 & 0.5 & 0.0100 & 1.05 & 0.92 & 1.15 \\
45 & 1.22 & 1.27 & 0.5 & 0.0100 & 0.98 & 1.02 & 0.96 \\
\hline
\end{tabular}

Table 18: Laboratory lift and drag data for the Blue Shark $\left(0-45^{\circ}\right)$

\begin{tabular}{|c|cccccccc|}
\hline $\begin{array}{c}\boldsymbol{\alpha} \\
\mathbf{(}{ }^{\circ}\end{array}$ & Lift (N) & Drag (N) & $\begin{array}{c}\text { Drag- } \\
\text { board }(\mathbf{N})\end{array}$ & $\mathbf{U}(\mathbf{m p s})$ & $\mathbf{A}\left(\mathbf{m}^{2}\right)$ & $\mathbf{C}_{\mathrm{L}}$ & $\mathbf{C}_{\mathbf{D}}$ & $\mathbf{C}_{\mathrm{L}} / \mathbf{C}_{\mathbf{D}}$ \\
\hline 0 & 0.01 & 0.44 & 0.00 & 0.5 & 0.0043 & 0.01 & 0.00 & 2.00 \\
5 & 0.24 & 0.49 & 0.05 & 0.5 & 0.0043 & 0.45 & 0.08 & 5.33 \\
10 & 0.47 & 0.53 & 0.09 & 0.5 & 0.0043 & 0.87 & 0.16 & 5.47 \\
15 & 0.60 & 0.59 & 0.15 & 0.5 & 0.0043 & 1.13 & 0.28 & 4.07 \\
20 & 0.66 & 0.68 & 0.24 & 0.5 & 0.0043 & 1.23 & 0.45 & 2.76 \\
25 & 0.66 & 0.78 & 0.34 & 0.5 & 0.0043 & 1.23 & 0.63 & 1.94 \\
30 & 0.59 & 0.82 & 0.38 & 0.5 & 0.0043 & 1.11 & 0.70 & 1.57 \\
35 & 0.60 & 0.87 & 0.43 & 0.5 & 0.0043 & 1.12 & 0.80 & 1.39 \\
40 & 0.58 & 0.97 & 0.53 & 0.5 & 0.0043 & 1.08 & 0.99 & 1.10 \\
45 & 0.56 & 1.02 & 0.58 & 0.5 & 0.0043 & 1.05 & 1.09 & 0.96 \\
\hline
\end{tabular}




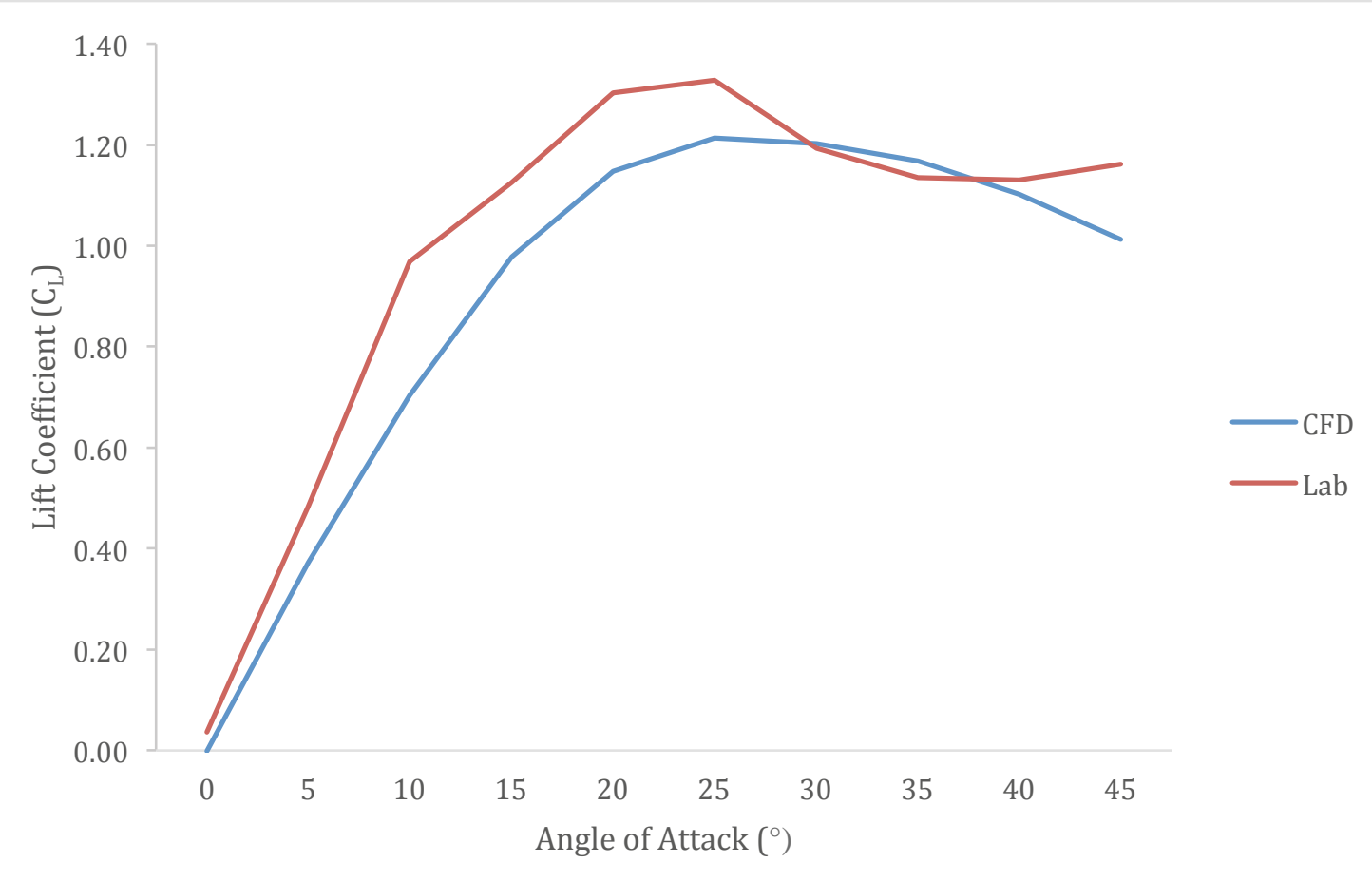

Figure 103: Coefficient of lift from laboratory and CFD data collected for the Fin Whale at ten angles of attack $\left(0-45^{\circ}\right)$

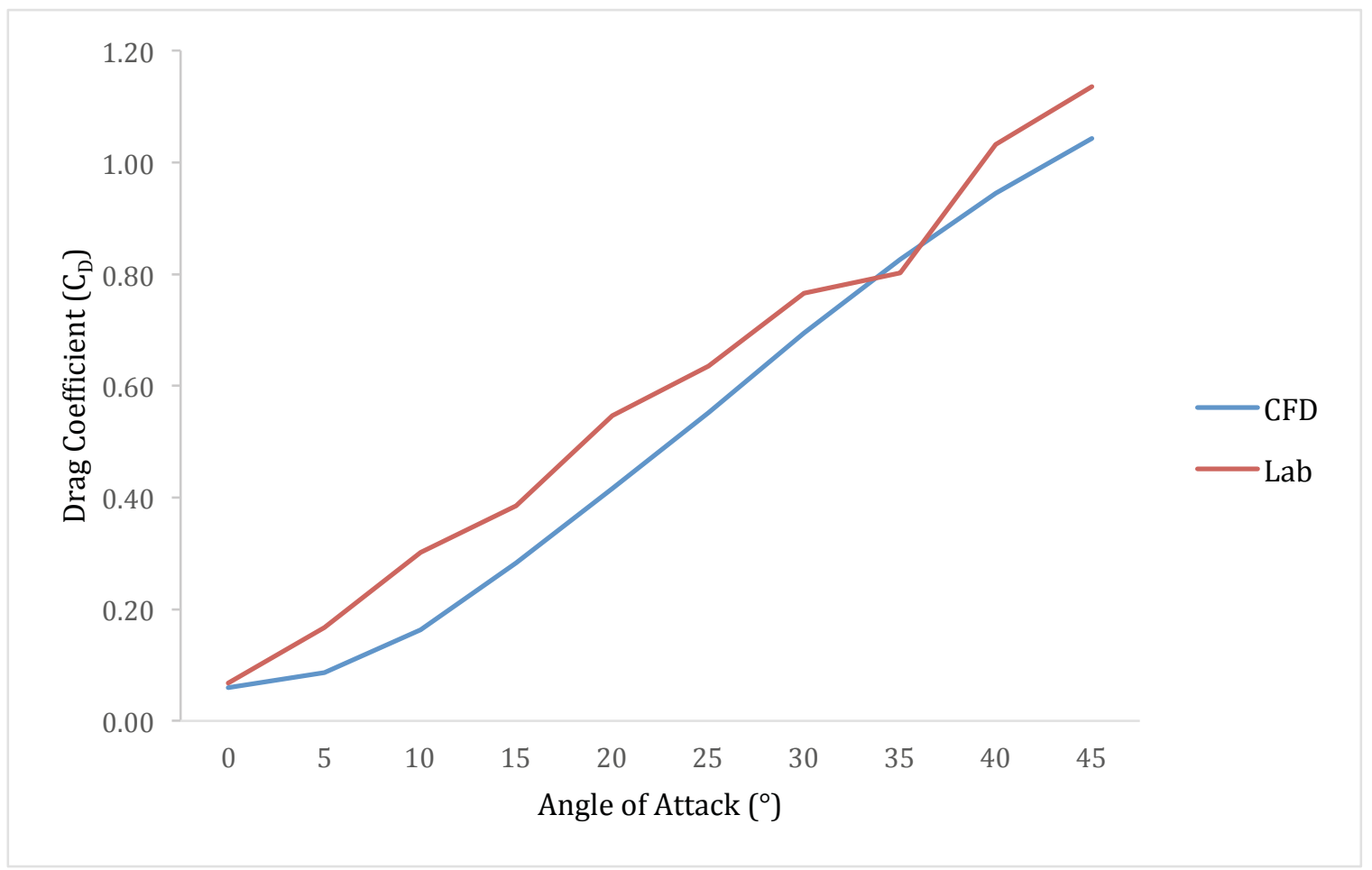

Figure 104: Coefficient of drag from laboratory and CFD data collected for the Fin Whale at ten angles of attack $\left(0-45^{\circ}\right)$ 
Table 19: CFD lift and drag data for the Fin Whale $\left(0-45^{\circ}\right)$

\begin{tabular}{|c|ccccccc|}
\hline $\boldsymbol{\alpha}\left({ }^{\circ}\right)$ & Lift (N) & Drag (N) & $\mathbf{U}(\mathbf{m p s})$ & $\mathbf{A}\left(\mathbf{m}^{2}\right)$ & $\mathrm{C}_{\mathrm{L}}$ & $\mathrm{C}_{\mathrm{D}}$ & $\mathrm{C}_{\mathrm{L}} / \mathrm{C}_{\mathrm{D}}$ \\
\hline 0 & 0.00 & 0.07 & 0.5 & 0.0090 & 0.00 & 0.06 & 0.02 \\
5 & 0.42 & 0.10 & 0.5 & 0.0090 & 0.37 & 0.09 & 4.33 \\
10 & 0.79 & 0.18 & 0.5 & 0.0090 & 0.70 & 0.16 & 4.30 \\
15 & 1.10 & 0.32 & 0.5 & 0.0090 & 0.98 & 0.28 & 3.46 \\
20 & 1.29 & 0.47 & 0.5 & 0.0090 & 1.15 & 0.42 & 2.76 \\
25 & 1.37 & 0.62 & 0.5 & 0.0090 & 1.21 & 0.55 & 2.20 \\
30 & 1.36 & 0.78 & 0.5 & 0.0090 & 1.20 & 0.69 & 1.73 \\
35 & 1.32 & 0.93 & 0.5 & 0.0090 & 1.17 & 0.83 & 1.41 \\
40 & 1.24 & 1.06 & 0.5 & 0.0090 & 1.10 & 0.94 & 1.17 \\
45 & 1.14 & 1.18 & 0.5 & 0.0090 & 1.01 & 1.04 & 0.97 \\
\hline
\end{tabular}

Table 20: Laboratory lift and drag data for the Fin Whale $\left(0-45^{\circ}\right)$

\begin{tabular}{|c|cccccccc|}
\hline $\begin{array}{c}\boldsymbol{\alpha} \\
\left.\mathbf{C}^{\circ}\right)\end{array}$ & Lift (N) & Drag (N) & $\begin{array}{c}\text { Drag- } \\
\text { board (N) }\end{array}$ & $\mathbf{U}(\mathbf{m p s})$ & $\mathbf{A}\left(\mathbf{m}^{2}\right)$ & $\mathbf{C}_{\mathrm{L}}$ & $\mathbf{C}_{\mathbf{D}}$ & $\mathbf{C}_{\mathrm{L}} / \mathbf{C}_{\mathbf{D}}$ \\
\hline 0 & 0.02 & 0.47 & 0.03 & 0.5 & 0.0038 & 0.04 & 0.07 & 0.54 \\
5 & 0.23 & 0.52 & 0.08 & 0.5 & 0.0038 & 0.49 & 0.17 & 2.91 \\
10 & 0.47 & 0.59 & 0.15 & 0.5 & 0.0038 & 0.97 & 0.30 & 3.21 \\
15 & 0.54 & 0.63 & 0.19 & 0.5 & 0.0038 & 1.13 & 0.39 & 2.92 \\
20 & 0.63 & 0.70 & 0.26 & 0.5 & 0.0038 & 1.30 & 0.55 & 2.38 \\
25 & 0.64 & 0.75 & 0.31 & 0.5 & 0.0038 & 1.33 & 0.64 & 2.09 \\
30 & 0.57 & 0.81 & 0.37 & 0.5 & 0.0038 & 1.19 & 0.77 & 1.56 \\
35 & 0.55 & 0.83 & 0.39 & 0.5 & 0.0038 & 1.14 & 0.80 & 1.42 \\
40 & 0.54 & 0.94 & 0.50 & 0.5 & 0.0038 & 1.13 & 1.03 & 1.10 \\
45 & 0.56 & 0.99 & 0.55 & 0.5 & 0.0038 & 1.16 & 1.14 & 1.02 \\
\hline
\end{tabular}




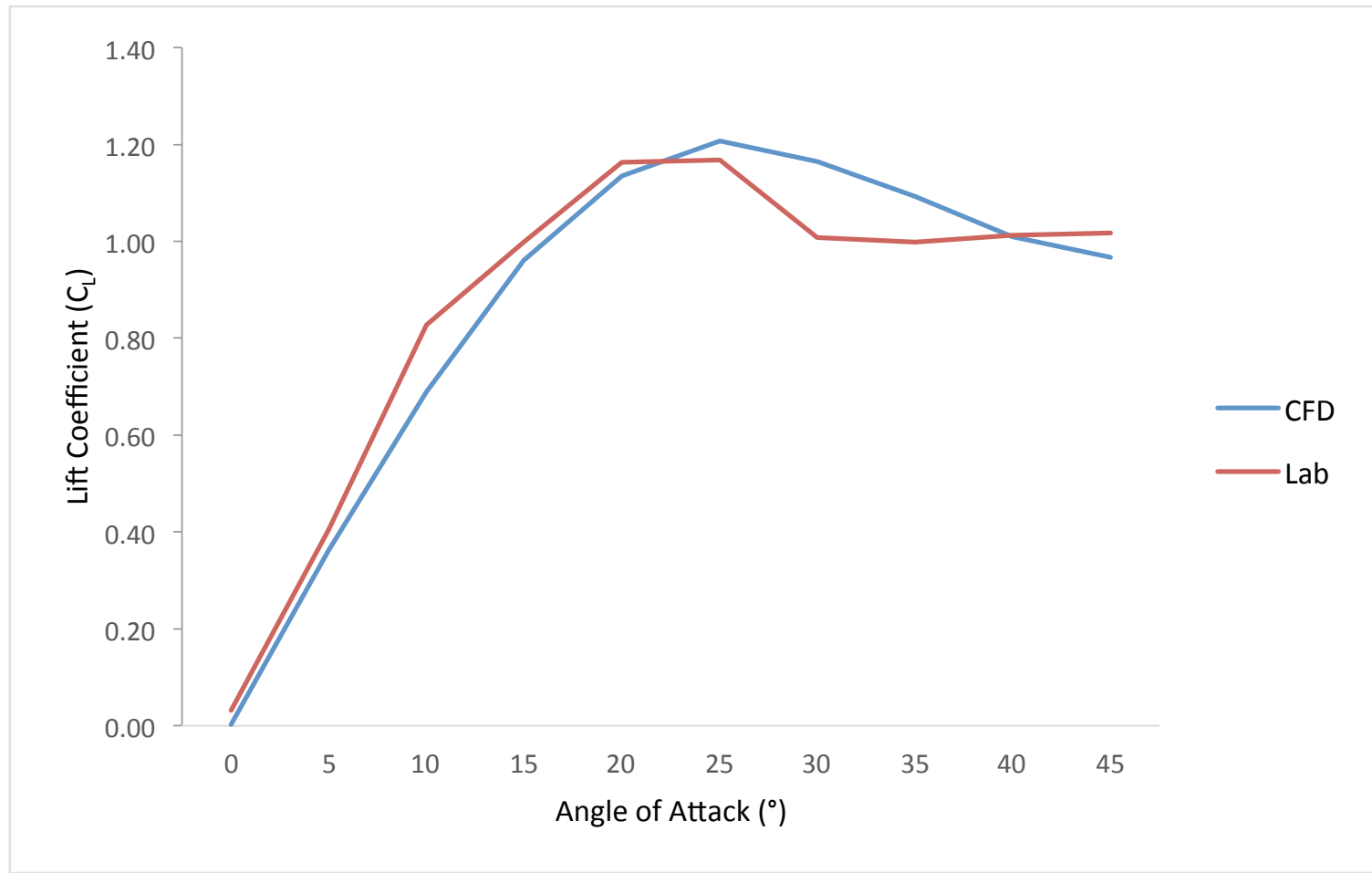

Figure 105: Coefficient of lift from laboratory and CFD data collected for the Dall's Porpoise at ten angles of attack $\left(0-45^{\circ}\right)$

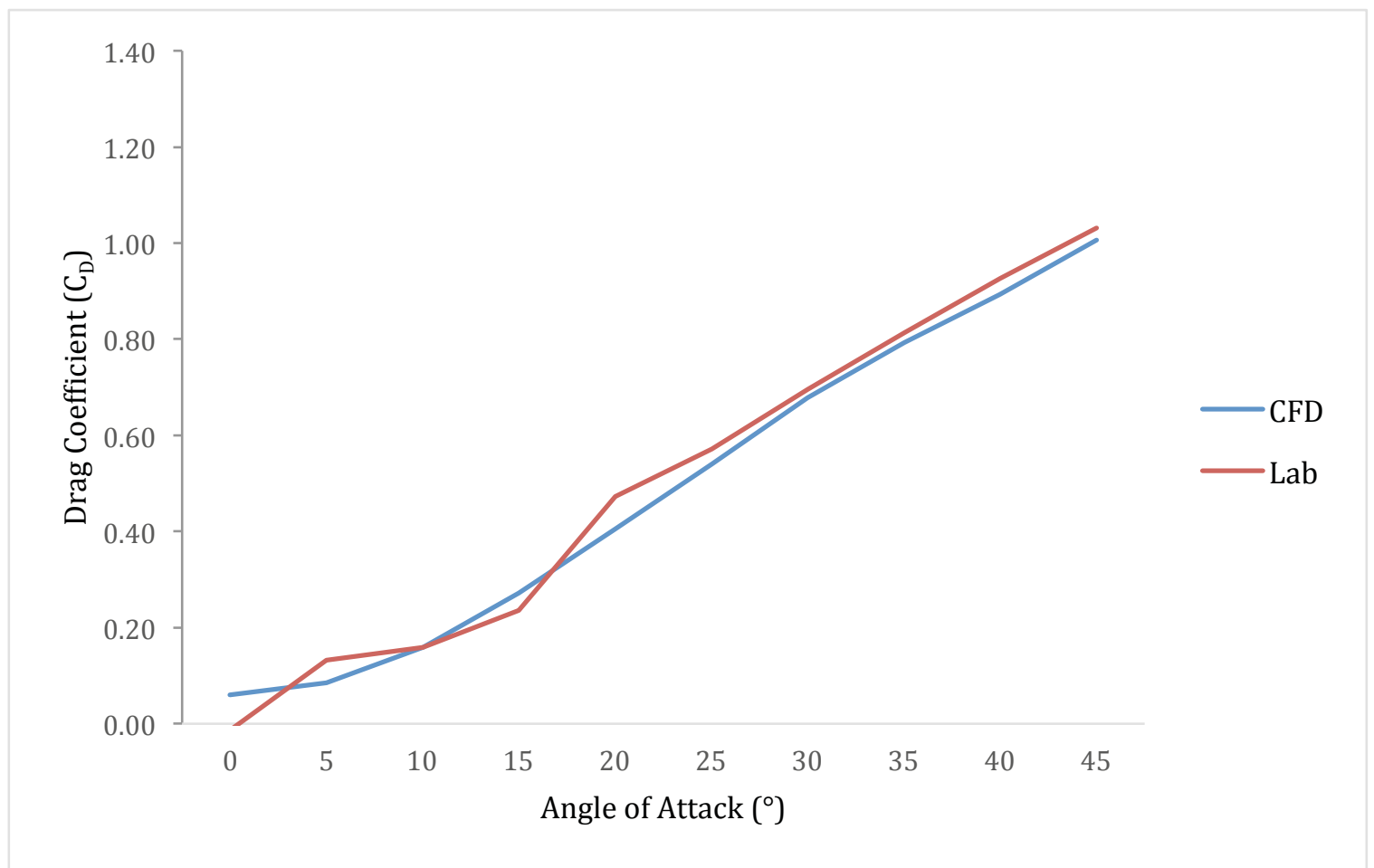

Figure 106: Coefficient of lift from laboratory and CFD data collected for the Dall's Porpoise at ten angles of attack $\left(0-45^{\circ}\right)$ 
Table 21: CFD lift and drag data for the Dall's Porpoise $\left(0-45^{\circ}\right)$

\begin{tabular}{|c|ccccccc|}
\hline $\begin{array}{c}\alpha \\
\mathbf{C}^{\circ}\end{array}$ & Lift (N) & Drag (N) & $\mathbf{U}(\mathbf{m p s})$ & $\mathbf{A}\left(\mathbf{m}^{2}\right)$ & $\mathbf{C}_{\mathrm{L}}$ & $\mathbf{C}_{\mathrm{D}}$ & $\mathrm{C}_{\mathrm{L}} / \mathrm{C}_{\mathrm{D}}$ \\
\hline 0 & 0.00 & 0.08 & 0.5 & 0.0104 & 0.00 & 0.06 & 0.04 \\
5 & 0.47 & 0.11 & 0.5 & 0.0104 & 0.36 & 0.09 & 4.25 \\
10 & 0.89 & 0.20 & 0.5 & 0.0104 & 0.69 & 0.16 & 4.36 \\
15 & 1.25 & 0.35 & 0.5 & 0.0104 & 0.96 & 0.27 & 3.53 \\
20 & 1.47 & 0.52 & 0.5 & 0.0104 & 1.14 & 0.41 & 2.81 \\
25 & 1.56 & 0.70 & 0.5 & 0.0104 & 1.21 & 0.54 & 2.24 \\
30 & 1.51 & 0.88 & 0.5 & 0.0104 & 1.17 & 0.68 & 1.72 \\
35 & 1.42 & 1.03 & 0.5 & 0.0104 & 1.09 & 0.79 & 1.38 \\
40 & 1.31 & 1.16 & 0.5 & 0.0104 & 1.01 & 0.89 & 1.13 \\
45 & 1.25 & 1.30 & 0.5 & 0.0104 & 0.97 & 1.01 & 0.96 \\
\hline
\end{tabular}

Table 22: Laboratory lift and drag data for the Dall's Porpoise $\left(0-45^{\circ}\right)$

\begin{tabular}{|c|cccccccc|}
\hline $\begin{array}{c}\alpha \\
\left({ }^{\circ}\right)\end{array}$ & Lift (N) & Drag (N) & $\begin{array}{c}\text { Drag- } \\
\text { board (N) }\end{array}$ & $\mathbf{U}(\mathbf{m p s})$ & $\mathbf{A}\left(\mathbf{m}^{2}\right)$ & $\mathbf{C}_{\mathrm{L}}$ & $\mathbf{C}_{\mathbf{D}}$ & $\mathbf{C}_{\mathbf{L}} / \mathbf{C}_{\mathbf{D}}$ \\
\hline 0 & 0.02 & 0.43 & -0.01 & 0.5 & 0.0044 & 0.03 & -0.01 & -2.33 \\
5 & 0.22 & 0.51 & 0.07 & 0.5 & 0.0044 & 0.40 & 0.13 & 3.07 \\
10 & 0.46 & 0.53 & 0.09 & 0.5 & 0.0044 & 0.83 & 0.16 & 5.20 \\
15 & 0.55 & 0.57 & 0.13 & 0.5 & 0.0044 & 1.00 & 0.24 & 4.23 \\
20 & 0.64 & 0.70 & 0.26 & 0.5 & 0.0044 & 1.16 & 0.47 & 2.46 \\
25 & 0.64 & 0.76 & 0.32 & 0.5 & 0.0044 & 1.17 & 0.57 & 2.04 \\
30 & 0.56 & 0.82 & 0.38 & 0.5 & 0.0044 & 1.01 & 0.70 & 1.45 \\
35 & 0.55 & 0.89 & 0.45 & 0.5 & 0.0044 & 1.00 & 0.81 & 1.23 \\
40 & 0.56 & 0.95 & 0.51 & 0.5 & 0.0044 & 1.01 & 0.93 & 1.09 \\
45 & 0.56 & 1.01 & 0.57 & 0.5 & 0.0044 & 1.02 & 1.03 & 0.99 \\
\hline
\end{tabular}




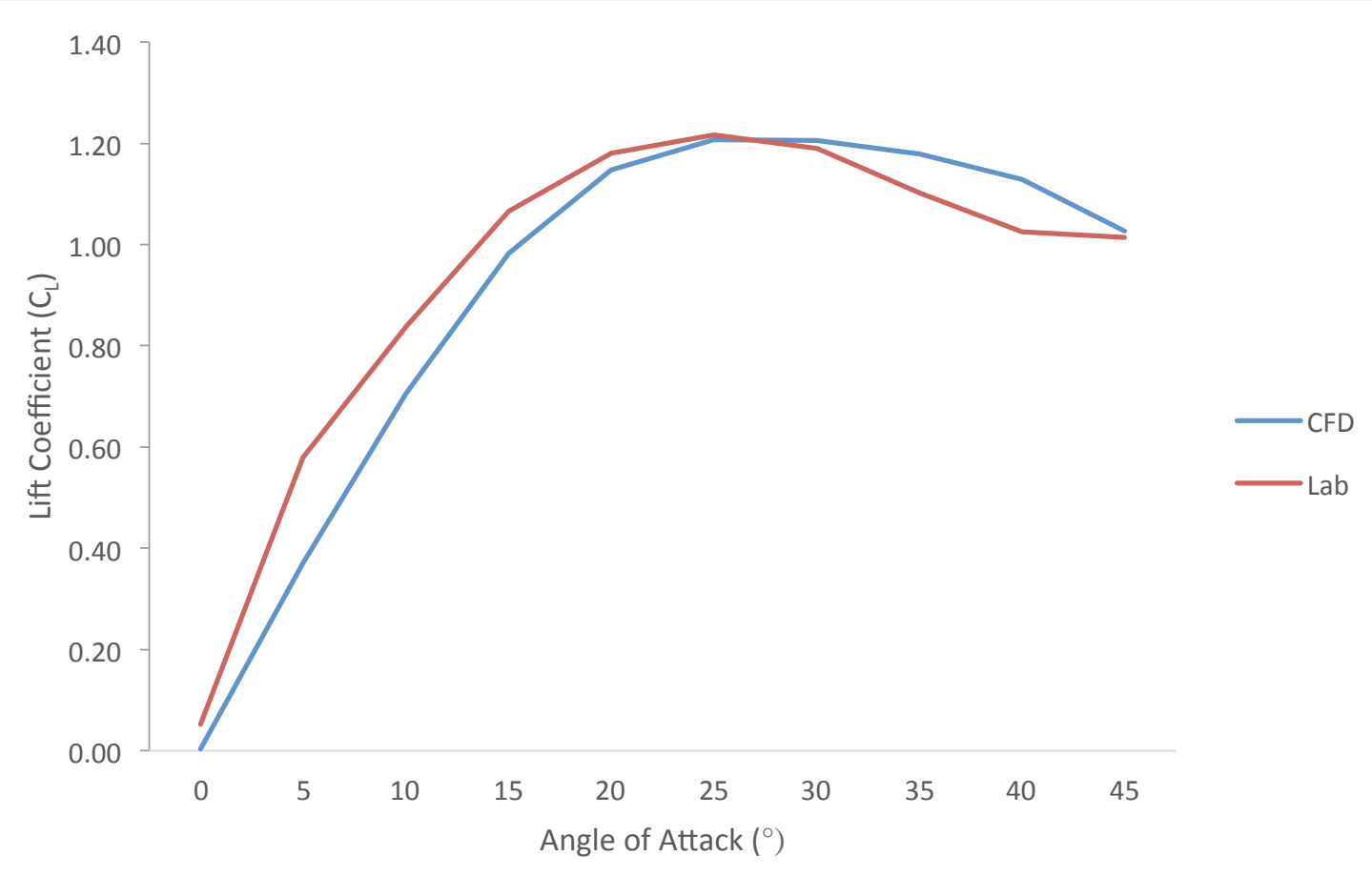

Figure 107: Coefficient of lift from laboratory and CFD data collected for the Spotted Dolphin at ten angles of attack $\left(0-45^{\circ}\right)$

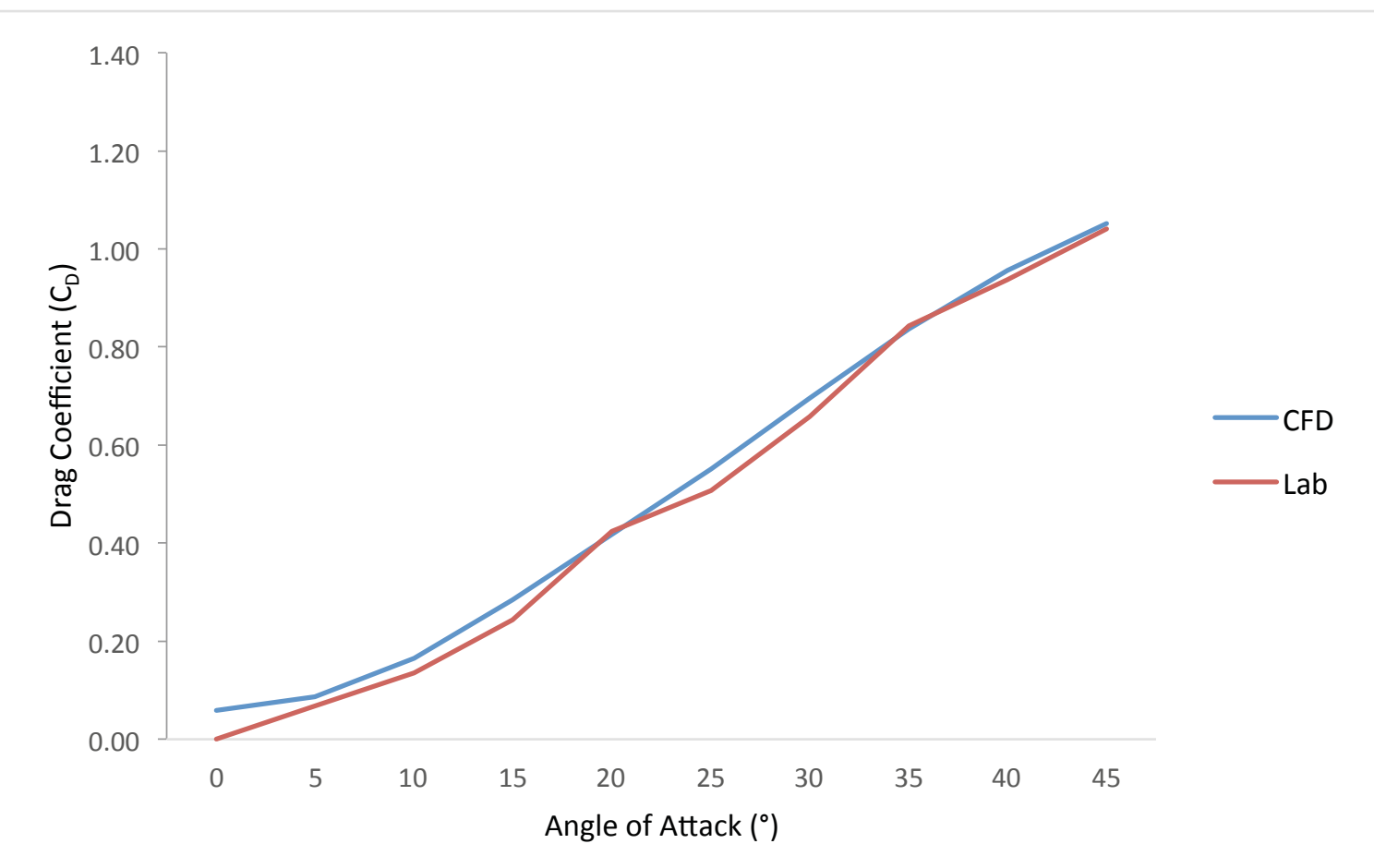

Figure 108: Coefficient of drag from laboratory and CFD data collected for the Spotted Dolphin at ten angles of attack $\left(0-45^{\circ}\right)$ 
Table 23: CFD lift and drag data for the Spotted Dolphin $\left(0-45^{\circ}\right)$

\begin{tabular}{|c|ccccccc|}
\hline $\begin{array}{c}\boldsymbol{\alpha} \\
\mathbf{(})\end{array}$ & Lift (N) & Drag (N) & $\mathbf{U}(\mathbf{m p s})$ & $\mathbf{A}\left(\mathbf{m}^{2}\right)$ & $\mathbf{C}_{\mathrm{L}}$ & $\mathbf{C}_{\mathrm{D}}$ & $\mathbf{C}_{\mathrm{L}} / \mathrm{C}_{\mathbf{D}}$ \\
\hline 0 & 0.00 & 0.07 & 0.5 & 0.0091 & 0.00 & 0.06 & 0.05 \\
5 & 0.42 & 0.10 & 0.5 & 0.0091 & 0.37 & 0.09 & 4.31 \\
10 & 0.80 & 0.19 & 0.5 & 0.0091 & 0.71 & 0.16 & 4.29 \\
15 & 1.12 & 0.32 & 0.5 & 0.0091 & 0.98 & 0.29 & 3.45 \\
20 & 1.30 & 0.47 & 0.5 & 0.0091 & 1.15 & 0.42 & 2.75 \\
25 & 1.37 & 0.63 & 0.5 & 0.0091 & 1.21 & 0.55 & 2.19 \\
30 & 1.37 & 0.79 & 0.5 & 0.0091 & 1.21 & 0.70 & 1.73 \\
35 & 1.34 & 0.95 & 0.5 & 0.0091 & 1.18 & 0.84 & 1.41 \\
40 & 1.28 & 1.08 & 0.5 & 0.0091 & 1.13 & 0.96 & 1.18 \\
45 & 1.17 & 1.19 & 0.5 & 0.0091 & 1.03 & 1.05 & 0.98 \\
\hline
\end{tabular}

Table 24: Laboratory lift and drag data for the Spotted Dolphin $\left(0-45^{\circ}\right)$

\begin{tabular}{|c|cccccccc|}
\hline $\begin{array}{c}\alpha \\
\left({ }^{\circ}\right)\end{array}$ & Lift (N) & Drag (N) & $\begin{array}{c}\text { Drag- } \\
\text { board (N) }\end{array}$ & $\mathbf{U}(\mathbf{m p s})$ & $\mathbf{A}\left(\mathbf{m}^{2}\right)$ & $\mathbf{C}_{\mathrm{L}}$ & $\mathbf{C}_{\mathrm{D}}$ & $\mathbf{C}_{\mathrm{L}} / \mathbf{C}_{\mathbf{D}}$ \\
\hline 0 & 0.03 & 0.44 & 0.00 & 0.5 & 0.0039 & 0.05 & 0.00 & 0.00 \\
5 & 0.28 & 0.50 & 0.06 & 0.5 & 0.0039 & 0.58 & 0.12 & 4.87 \\
10 & 0.41 & 0.53 & 0.09 & 0.5 & 0.0039 & 0.84 & 0.18 & 4.76 \\
15 & 0.52 & 0.57 & 0.13 & 0.5 & 0.0039 & 1.07 & 0.26 & 4.04 \\
20 & 0.58 & 0.65 & 0.21 & 0.5 & 0.0039 & 1.20 & 0.42 & 2.83 \\
25 & 0.62 & 0.69 & 0.25 & 0.5 & 0.0039 & 1.29 & 0.51 & 2.53 \\
30 & 0.58 & 0.76 & 0.32 & 0.5 & 0.0039 & 1.19 & 0.66 & 1.81 \\
35 & 0.53 & 0.85 & 0.41 & 0.5 & 0.0039 & 1.10 & 0.84 & 1.31 \\
40 & 0.50 & 0.89 & 0.45 & 0.5 & 0.0039 & 1.03 & 0.94 & 1.09 \\
45 & 0.49 & 0.94 & 0.50 & 0.5 & 0.0039 & 1.02 & 1.04 & 0.98 \\
\hline
\end{tabular}




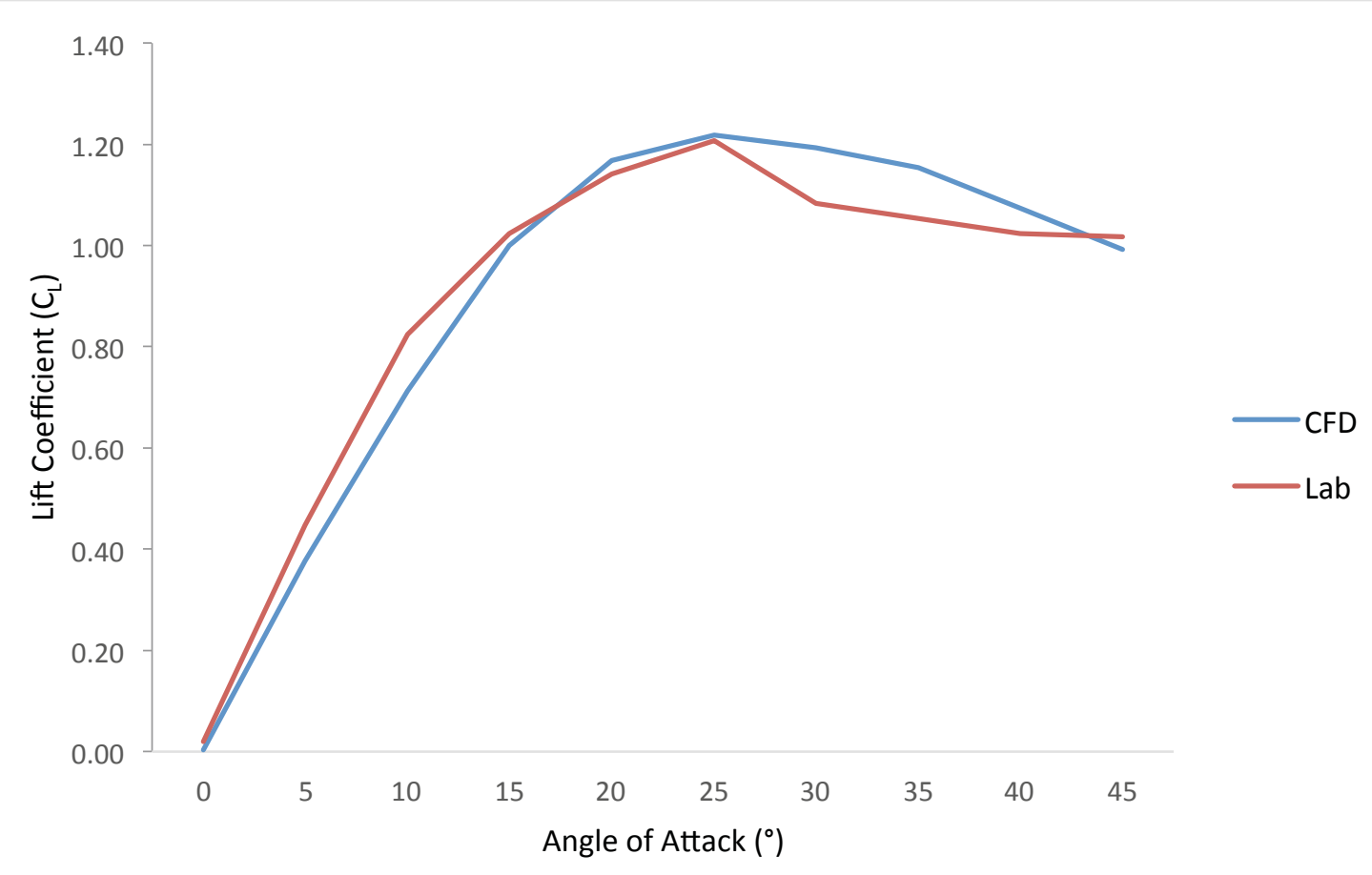

Figure 109: Coefficient of lift from laboratory and CFD data collected for the Killer Whale at ten angles of attack $\left(0-45^{\circ}\right)$

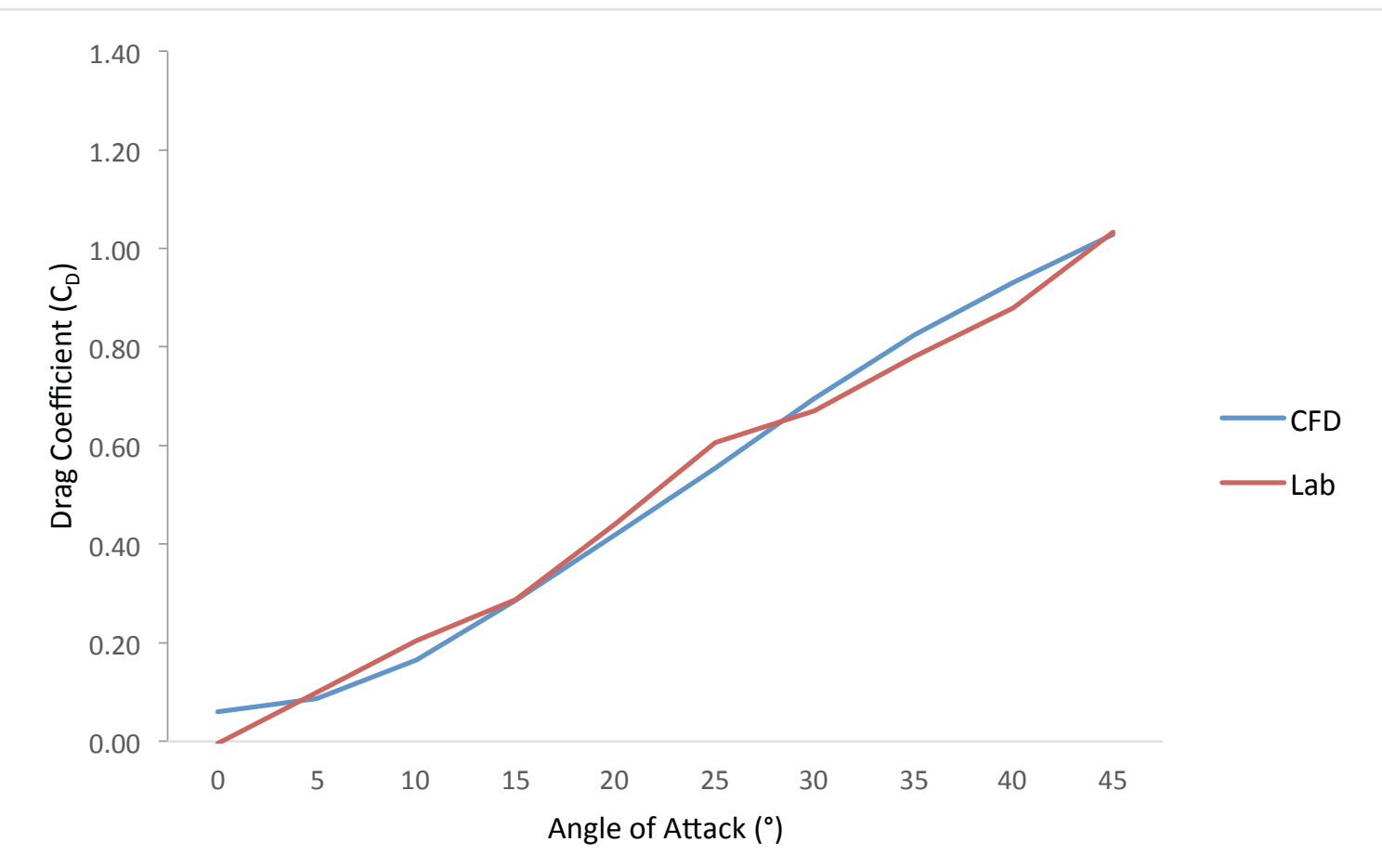

Figure 110: Coefficient of drag from laboratory and CFD data collected for the Killer Whale at ten angles of attack $\left(0-45^{\circ}\right)$ 
Table 25: CFD lift and drag data for the Orca $\left(0-45^{\circ}\right)$

\begin{tabular}{|c|ccccccc|}
\hline $\begin{array}{c}\boldsymbol{\alpha} \\
\mathbf{(})\end{array}$ & $\begin{array}{c}\text { Lift } \\
(\mathbf{N})\end{array}$ & Drag (N) & $\mathbf{U}(\mathbf{m p s})$ & $\mathbf{A}\left(\mathbf{m}^{2}\right)$ & $\mathbf{C}_{\mathrm{L}}$ & $\mathbf{C}_{\mathbf{D}}$ & $\mathbf{C}_{\mathrm{L}} / \mathbf{C}_{\mathbf{D}}$ \\
\hline 0 & 0.00 & 0.07 & 0.5 & 0.0095 & 0.00 & 0.06 & 0.06 \\
5 & 0.45 & 0.10 & 0.5 & 0.0095 & 0.38 & 0.09 & 4.31 \\
10 & 0.84 & 0.20 & 0.5 & 0.0095 & 0.71 & 0.17 & 4.32 \\
15 & 1.18 & 0.34 & 0.5 & 0.0095 & 1.00 & 0.29 & 3.50 \\
20 & 1.38 & 0.50 & 0.5 & 0.0095 & 1.17 & 0.42 & 2.78 \\
25 & 1.44 & 0.66 & 0.5 & 0.0095 & 1.22 & 0.56 & 2.20 \\
30 & 1.41 & 0.82 & 0.5 & 0.0095 & 1.20 & 0.70 & 1.72 \\
35 & 1.36 & 0.97 & 0.5 & 0.0095 & 1.16 & 0.82 & 1.40 \\
40 & 1.27 & 1.10 & 0.5 & 0.0095 & 1.08 & 0.93 & 1.15 \\
45 & 1.17 & 1.21 & 0.5 & 0.0095 & 0.99 & 1.03 & 0.97 \\
\hline
\end{tabular}

Table 26: Laboratory lift and drag data for the Orca $\left(0-45^{\circ}\right)$

\begin{tabular}{|c|cccccccc|}
\hline $\begin{array}{c}\alpha \\
\left({ }^{\circ}\right)\end{array}$ & Lift (N) & Drag (N) & $\begin{array}{c}\text { Drag- } \\
\text { board (N) }\end{array}$ & $\mathbf{U}(\mathbf{m p s})$ & $\mathbf{A}(\mathbf{m} 2)$ & $C_{L}$ & $C_{D}$ & $C_{L} / C_{D}$ \\
\hline 0 & 0.01 & 0.44 & 0.00 & 0.5 & 0.0040 & 0.02 & 0.00 & -4.00 \\
5 & 0.23 & 0.49 & 0.05 & 0.5 & 0.0040 & 0.45 & 0.10 & 4.50 \\
10 & 0.42 & 0.54 & 0.10 & 0.5 & 0.0040 & 0.83 & 0.20 & 4.05 \\
15 & 0.52 & 0.59 & 0.15 & 0.5 & 0.0040 & 1.02 & 0.29 & 3.55 \\
20 & 0.58 & 0.66 & 0.22 & 0.5 & 0.0040 & 1.14 & 0.44 & 2.58 \\
25 & 0.61 & 0.75 & 0.31 & 0.5 & 0.0040 & 1.21 & 0.61 & 1.99 \\
30 & 0.55 & 0.78 & 0.34 & 0.5 & 0.0040 & 1.08 & 0.67 & 1.61 \\
35 & 0.53 & 0.83 & 0.39 & 0.5 & 0.0040 & 1.05 & 0.78 & 1.35 \\
40 & 0.52 & 0.88 & 0.44 & 0.5 & 0.0040 & 1.02 & 0.88 & 1.16 \\
45 & 0.51 & 0.96 & 0.52 & 0.5 & 0.0040 & 1.02 & 1.03 & 0.99 \\
\hline
\end{tabular}




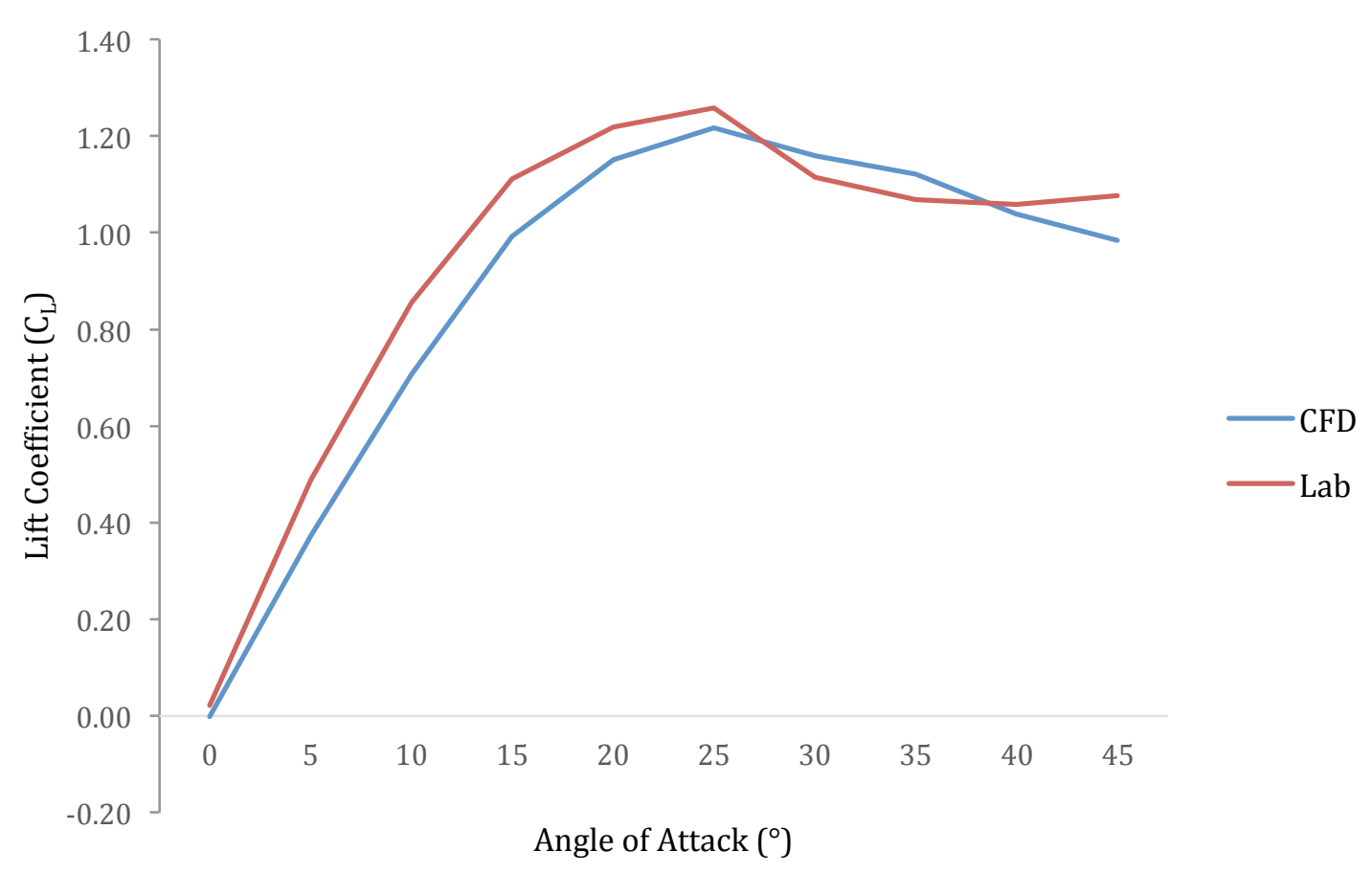

Figure 111: Coefficient of lift from laboratory and CFD data collected for the Shortfin Mako at ten angles of attack $\left(0-45^{\circ}\right)$

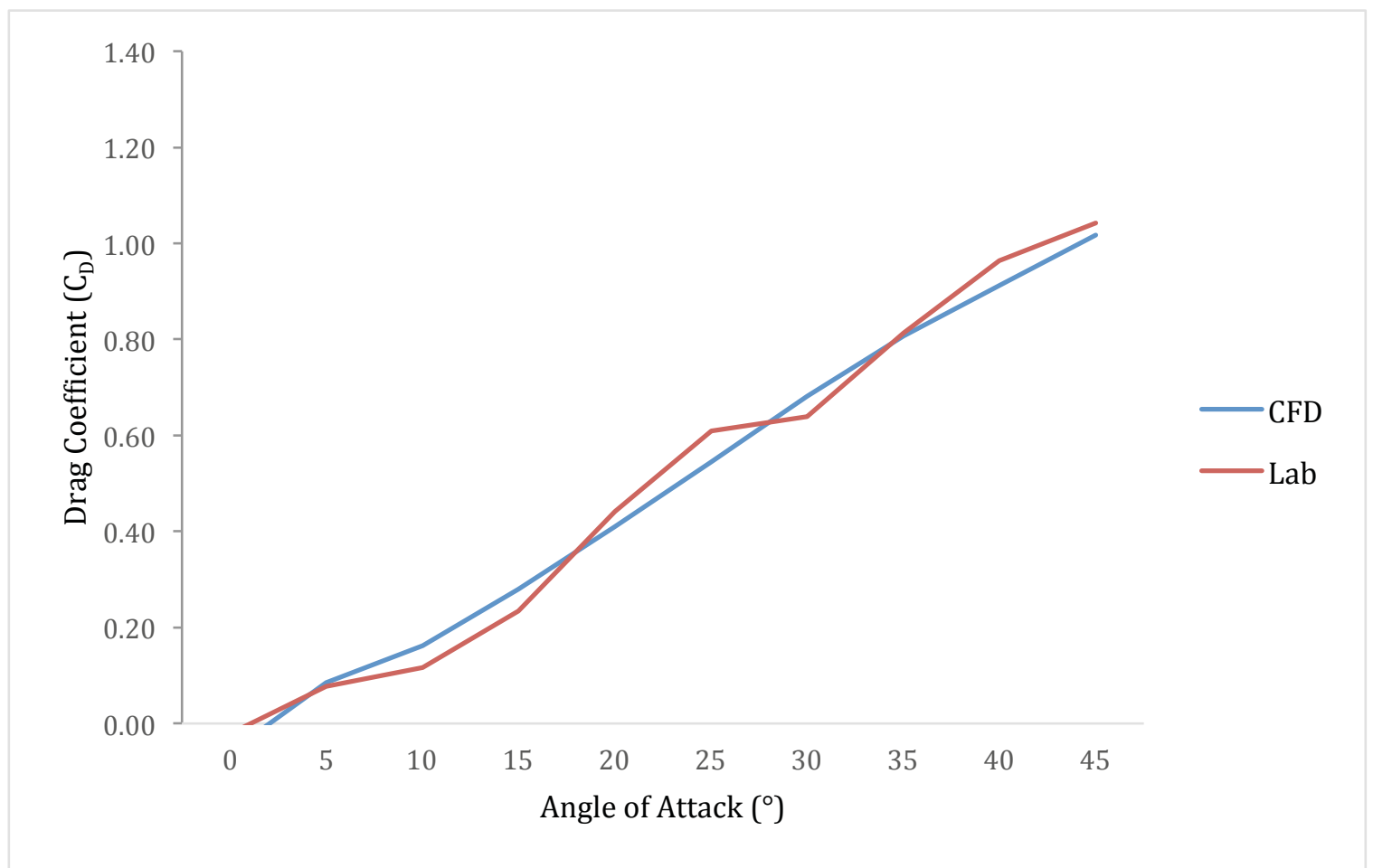

Figure 112: Coefficient of drag from laboratory and CFD data collected for the Shortfin Mako at ten angles of attack $\left(0-45^{\circ}\right)$ 
Table 27: CFD lift and drag data for the Shortfin Mako $\left(0-45^{\circ}\right)$

\begin{tabular}{|c|ccccccc|}
\hline $\begin{array}{c}\boldsymbol{\alpha} \\
\mathbf{(}{ }^{\circ}\end{array}$ & Lift (N) & Drag (N) & $\mathbf{U}(\mathbf{m p s})$ & $\mathbf{A}\left(\mathbf{m}^{2}\right)$ & $\mathbf{C}_{\mathrm{L}}$ & $\mathbf{C}_{\mathbf{D}}$ & $\mathbf{C}_{\mathrm{L}} / \mathbf{C}_{\mathbf{D}}$ \\
\hline 0 & 0.00 & -0.08 & 0.5 & 0.0109 & 0.00 & 0.06 & 0.03 \\
5 & 0.51 & 0.12 & 0.5 & 0.0109 & 0.37 & 0.09 & 4.35 \\
10 & 0.96 & 0.22 & 0.5 & 0.0109 & 0.71 & 0.16 & 4.37 \\
15 & 1.35 & 0.38 & 0.5 & 0.0109 & 0.99 & 0.28 & 3.55 \\
20 & 1.57 & 0.56 & 0.5 & 0.0109 & 1.15 & 0.41 & 2.80 \\
25 & 1.66 & 0.74 & 0.5 & 0.0109 & 1.22 & 0.55 & 2.23 \\
30 & 1.58 & 0.93 & 0.5 & 0.0109 & 1.16 & 0.68 & 1.70 \\
35 & 1.52 & 1.10 & 0.5 & 0.0109 & 1.12 & 0.81 & 1.39 \\
40 & 1.41 & 1.24 & 0.5 & 0.0109 & 1.04 & 0.91 & 1.14 \\
45 & 1.34 & 1.39 & 0.5 & 0.0109 & 0.98 & 1.02 & 0.97 \\
\hline
\end{tabular}

Table 28: Laboratory lift and drag data for the Shortfin Mako $\left(0-45^{\circ}\right)$

\begin{tabular}{|c|cccccccc|}
\hline $\begin{array}{c}\alpha \\
\left({ }^{\circ}\right)\end{array}$ & Lift (N) & Drag (N) & $\begin{array}{c}\text { Drag- } \\
\text { board (N) }\end{array}$ & $\mathbf{U}(\mathbf{m p s})$ & $\mathbf{A}\left(\mathbf{m}^{2}\right)$ & $\mathbf{C}_{\mathrm{L}}$ & $\mathbf{C}_{\mathbf{D}}$ & $\mathbf{C}_{\mathrm{L}} / \mathbf{C}_{\mathbf{D}}$ \\
\hline 0 & 0.01 & 0.45 & 0.01 & 0.5 & 0.0046 & 0.02 & 0.01 & 1.67 \\
5 & 0.27 & 0.49 & 0.05 & 0.5 & 0.0046 & 0.46 & 0.09 & 5.10 \\
10 & 0.47 & 0.52 & 0.08 & 0.5 & 0.0046 & 0.81 & 0.14 & 5.88 \\
15 & 0.64 & 0.58 & 0.14 & 0.5 & 0.0046 & 1.11 & 0.23 & 4.76 \\
20 & 0.71 & 0.70 & 0.26 & 0.5 & 0.0046 & 1.22 & 0.44 & 2.76 \\
25 & 0.73 & 0.79 & 0.35 & 0.5 & 0.0046 & 1.26 & 0.61 & 2.06 \\
30 & 0.65 & 0.81 & 0.37 & 0.5 & 0.0046 & 1.12 & 0.64 & 1.74 \\
35 & 0.62 & 0.91 & 0.47 & 0.5 & 0.0046 & 1.07 & 0.81 & 1.31 \\
40 & 0.61 & 1.00 & 0.56 & 0.5 & 0.0046 & 1.06 & 0.97 & 1.10 \\
45 & 0.62 & 1.04 & 0.60 & 0.5 & 0.0046 & 1.08 & 1.04 & 1.03 \\
\hline
\end{tabular}




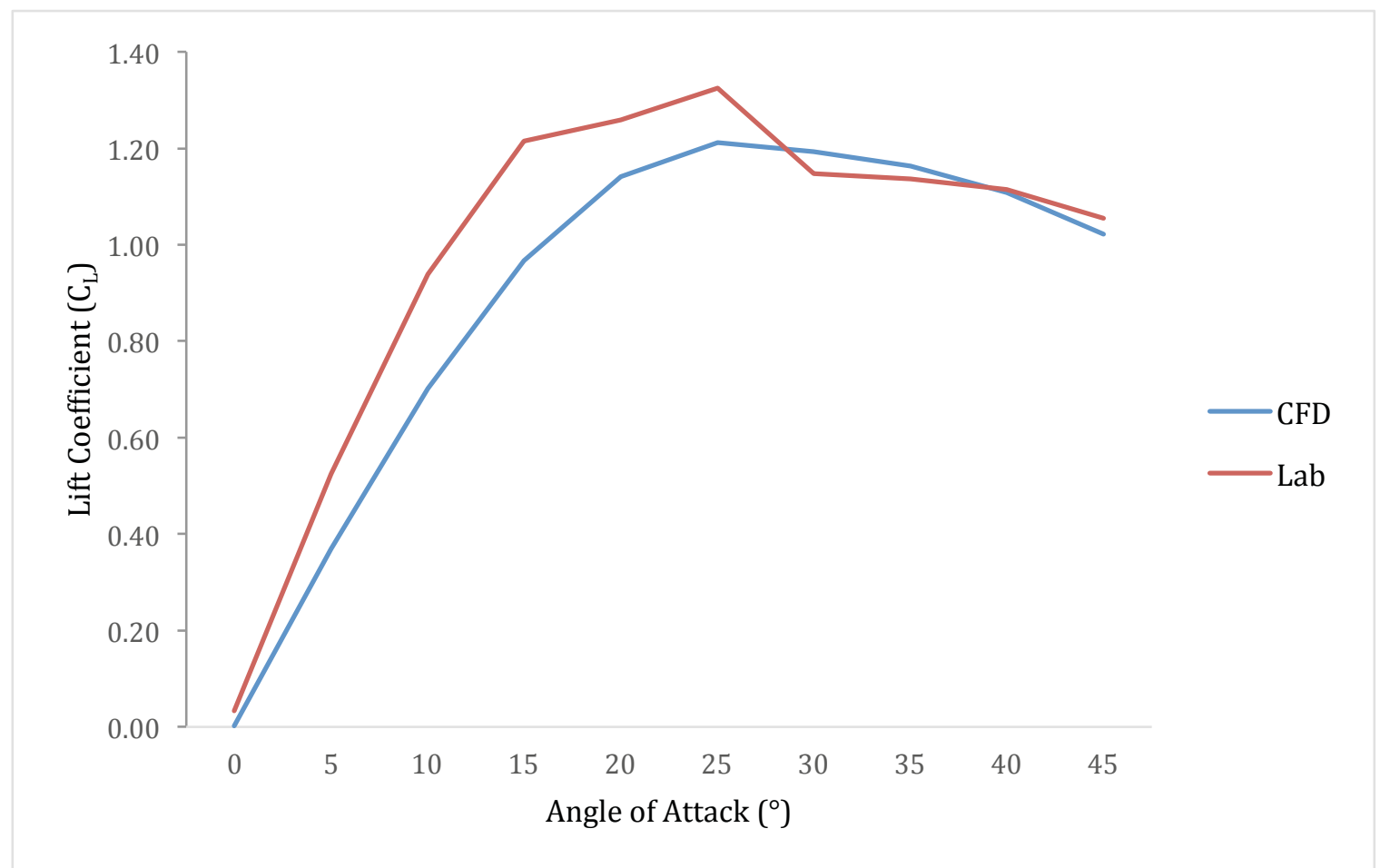

Figure 113: Coefficient of lift from laboratory and CFD data collected for the Swordfish at ten angles of attack $\left(0-45^{\circ}\right)$

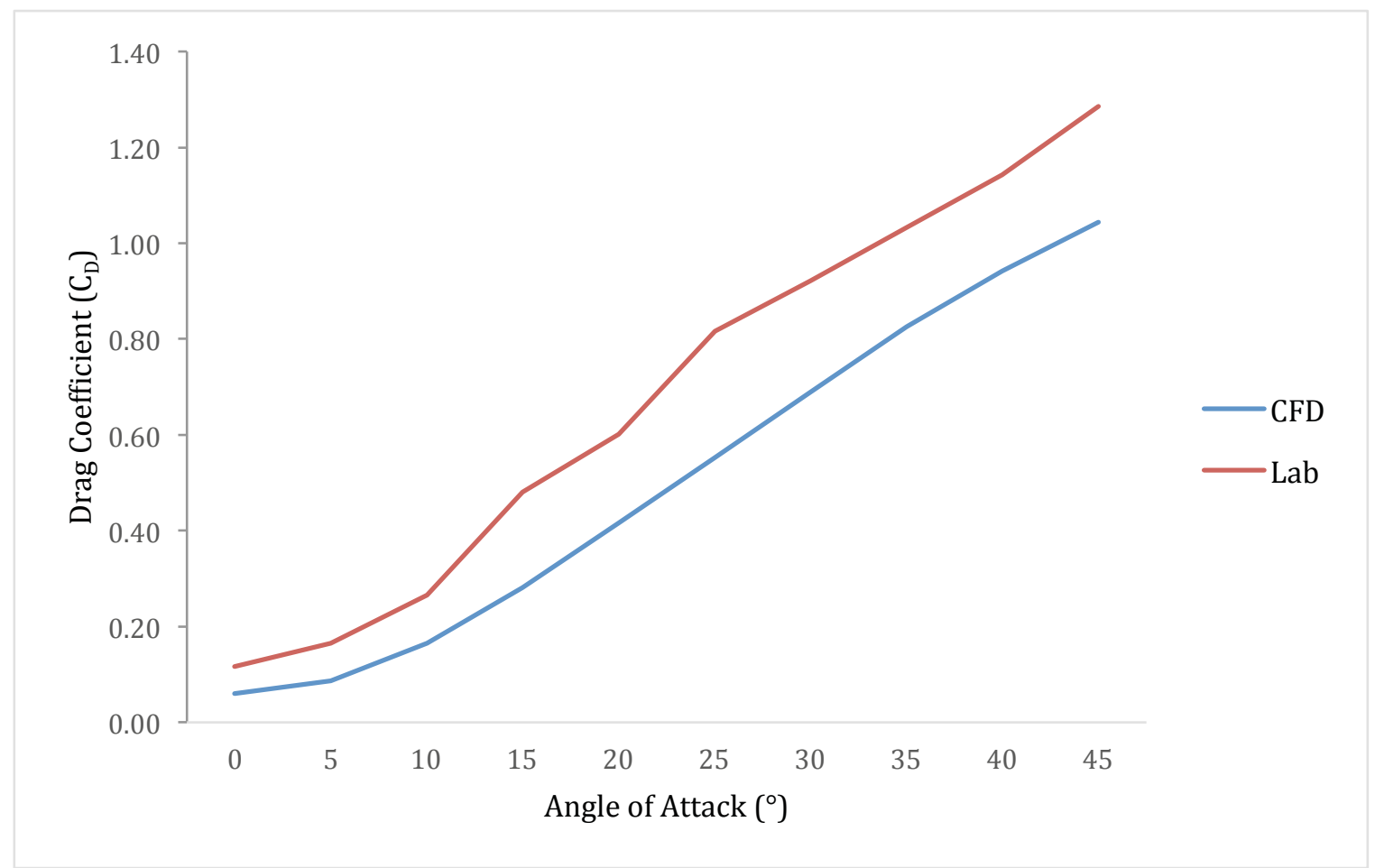

Figure 114: Coefficient of drag from laboratory and CFD data collected for the Swordfish at ten angles of attack $\left(0-45^{\circ}\right)$ 
Table 29: CFD lift and drag data for the Swordfish $\left(0-45^{\circ}\right)$

\begin{tabular}{|c|ccccccc|}
\hline $\begin{array}{c}\boldsymbol{\alpha} \\
\left.\mathbf{(}^{\circ}\right)\end{array}$ & Lift (N) & Drag (N) & $\mathbf{U}(\mathbf{m p s})$ & $\mathbf{A}\left(\mathbf{m}^{2}\right)$ & $\mathbf{C}_{\mathrm{L}}$ & $\mathbf{C}_{\mathbf{D}}$ & $\mathbf{C}_{\mathrm{L}} / \mathbf{C}_{\mathbf{D}}$ \\
\hline 0 & 0.00 & 0.06 & 0.5 & 0.0085 & 0.00 & 0.06 & -0.04 \\
5 & 0.39 & 0.09 & 0.5 & 0.0085 & 0.37 & 0.09 & 4.30 \\
10 & 0.75 & 0.18 & 0.5 & 0.0085 & 0.70 & 0.17 & 4.25 \\
15 & 1.03 & 0.30 & 0.5 & 0.0085 & 0.97 & 0.28 & 3.43 \\
20 & 1.21 & 0.44 & 0.5 & 0.0085 & 1.14 & 0.42 & 2.74 \\
25 & 1.29 & 0.59 & 0.5 & 0.0085 & 1.21 & 0.55 & 2.19 \\
30 & 1.27 & 0.73 & 0.5 & 0.0085 & 1.19 & 0.69 & 1.73 \\
35 & 1.24 & 0.88 & 0.5 & 0.0085 & 1.16 & 0.83 & 1.41 \\
40 & 1.18 & 1.00 & 0.5 & 0.0085 & 1.11 & 0.94 & 1.18 \\
45 & 1.09 & 1.11 & 0.5 & 0.0085 & 1.02 & 1.04 & 0.98 \\
\hline
\end{tabular}

Table 30: Laboratory lift and drag data for the Swordfish $\left(0-45^{\circ}\right)$

\begin{tabular}{|c|cccccccc|}
\hline $\begin{array}{c}\boldsymbol{\alpha} \\
\mathbf{(})\end{array}$ & Lift (N) & Drag (N) & $\begin{array}{c}\text { Drag- } \\
\text { board (N) }\end{array}$ & $\mathbf{U}(\mathbf{m p s})$ & $\mathbf{A}\left(\mathbf{m}^{2}\right)$ & $\mathbf{C}_{\mathrm{L}}$ & $\mathrm{C}_{\mathrm{D}}$ & $\mathrm{C}_{\mathrm{L}} / \mathbf{C}_{\mathrm{D}}$ \\
\hline 0 & 0.02 & 0.48 & 0.04 & 0.5 & 0.0036 & 0.03 & 0.08 & 0.43 \\
5 & 0.24 & 0.52 & 0.08 & 0.5 & 0.0036 & 0.53 & 0.17 & 3.17 \\
10 & 0.43 & 0.56 & 0.12 & 0.5 & 0.0036 & 0.94 & 0.27 & 3.54 \\
15 & 0.55 & 0.66 & 0.22 & 0.5 & 0.0036 & 1.22 & 0.48 & 2.53 \\
20 & 0.57 & 0.71 & 0.27 & 0.5 & 0.0036 & 1.26 & 0.60 & 2.09 \\
25 & 0.60 & 0.79 & 0.35 & 0.5 & 0.0036 & 1.33 & 0.76 & 1.74 \\
30 & 0.52 & 0.83 & 0.39 & 0.5 & 0.0036 & 1.15 & 0.87 & 1.32 \\
35 & 0.52 & 0.88 & 0.44 & 0.5 & 0.0036 & 1.14 & 0.97 & 1.18 \\
40 & 0.51 & 0.93 & 0.49 & 0.5 & 0.0036 & 1.12 & 1.09 & 1.03 \\
45 & 0.48 & 0.96 & 0.52 & 0.5 & 0.0036 & 1.06 & 1.16 & 0.91 \\
\hline
\end{tabular}




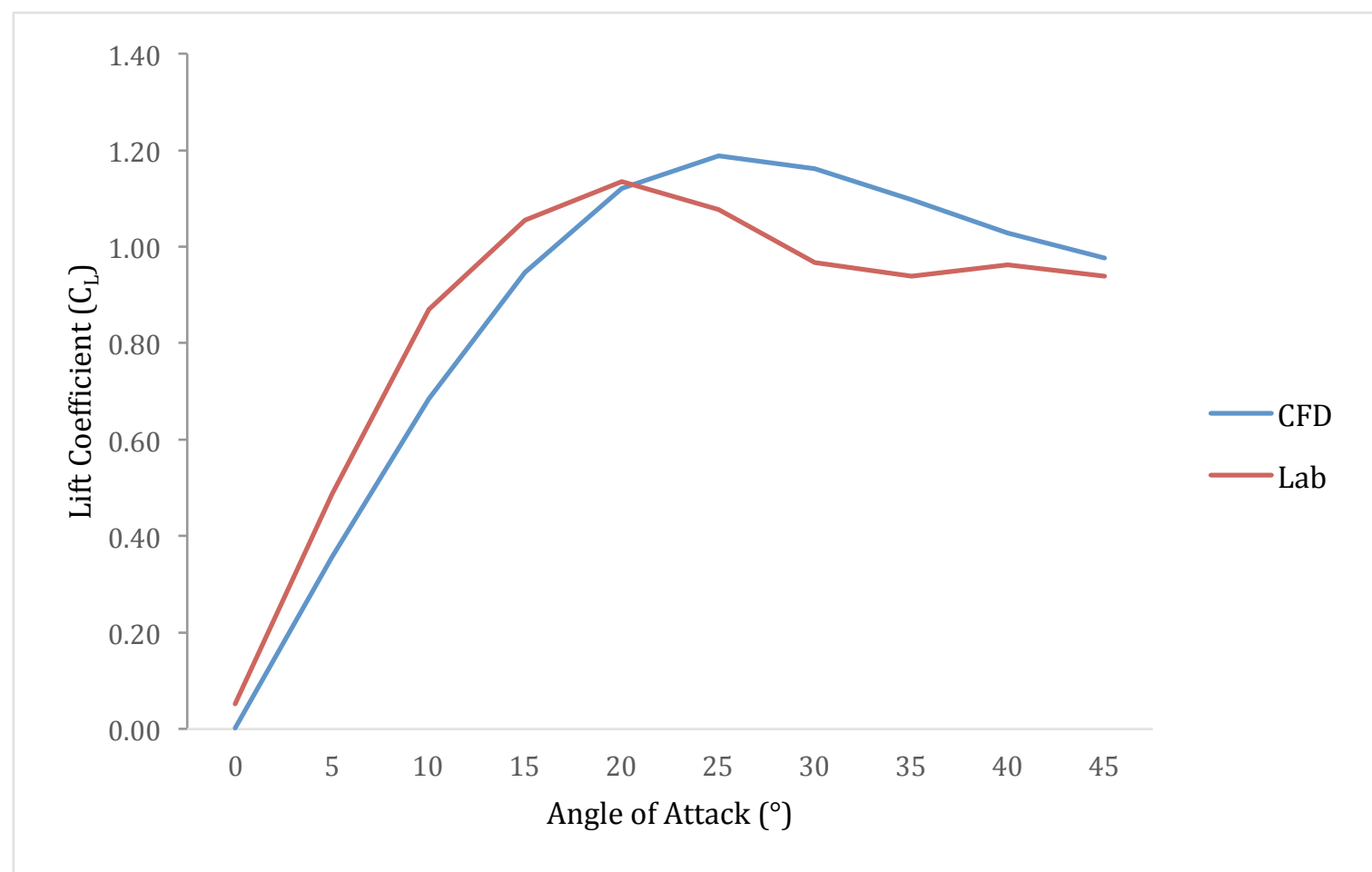

Figure 115: Coefficient of lift from experimental and CFD data collected for the Marlin at ten angles of attack $\left(0-45^{\circ}\right)$

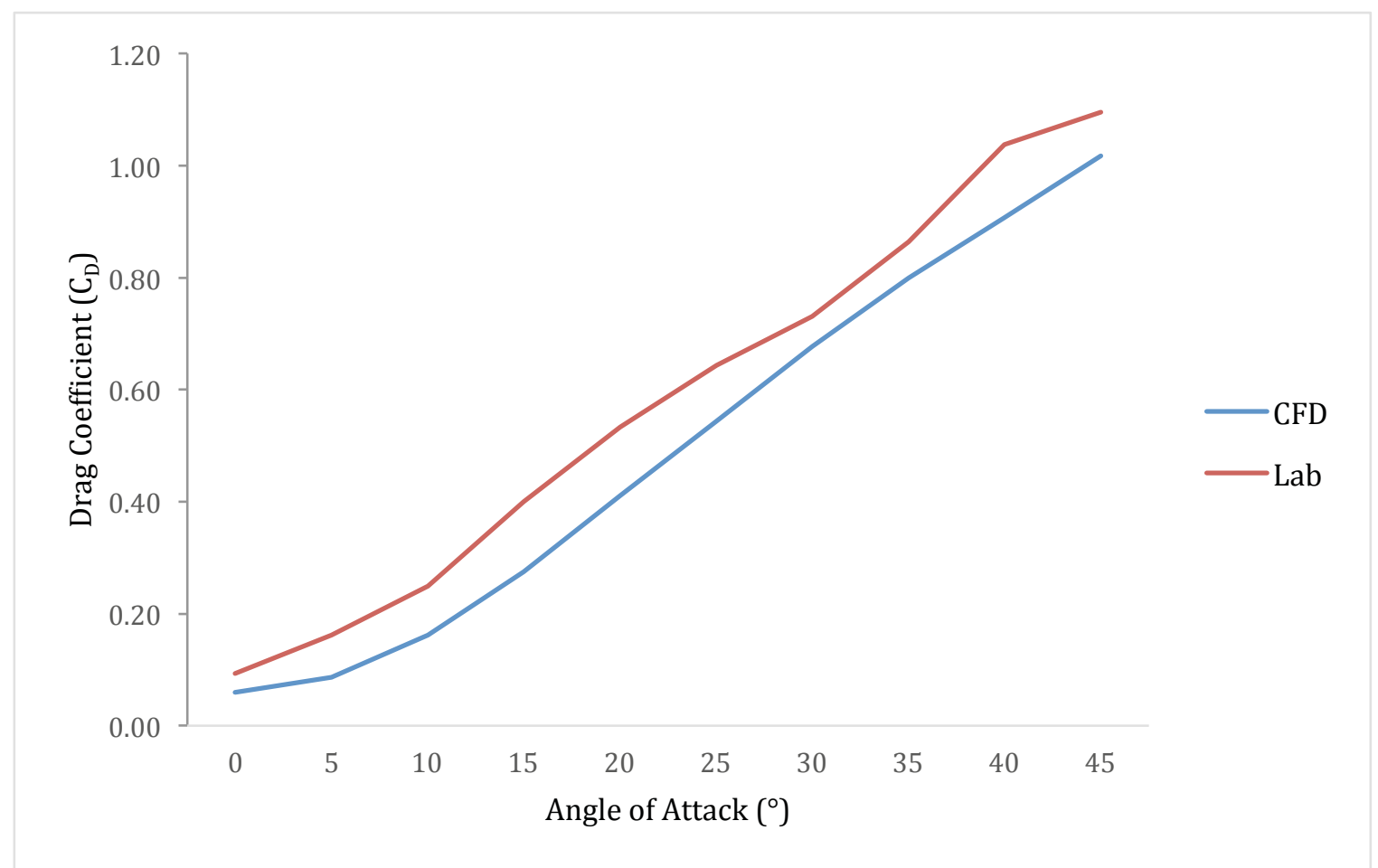

Figure 116: Coefficient of drag from experimental and CFD data collected for the Marlin at ten angles of attack $\left(0-45^{\circ}\right)$ 
Table 31: CFD lift and drag data for the Marlin $\left(0-45^{\circ}\right)$

\begin{tabular}{|c|ccccccc|}
\hline $\begin{array}{c}\boldsymbol{\alpha} \\
\mathbf{C}^{\circ}\end{array}$ & Lift (N) & Drag (N) & $\mathbf{U}(\mathbf{m p s})$ & $\mathbf{A}\left(\mathbf{m}^{2}\right)$ & $\mathbf{C}_{\mathrm{L}}$ & $\mathbf{C}_{\mathrm{D}}$ & $\mathbf{C}_{\mathrm{L}} / \mathbf{C}_{\mathbf{D}}$ \\
\hline 0 & 0.00 & 0.06 & 0.5 & 0.0081 & 0.00 & 0.06 & 0.02 \\
5 & 0.36 & 0.09 & 0.5 & 0.0081 & 0.36 & 0.09 & 4.11 \\
10 & 0.69 & 0.16 & 0.5 & 0.0081 & 0.68 & 0.16 & 4.22 \\
15 & 0.96 & 0.28 & 0.5 & 0.0081 & 0.95 & 0.28 & 3.44 \\
20 & 1.13 & 0.41 & 0.5 & 0.0081 & 1.12 & 0.41 & 2.73 \\
25 & 1.20 & 0.55 & 0.5 & 0.0081 & 1.19 & 0.54 & 2.19 \\
30 & 1.17 & 0.68 & 0.5 & 0.0081 & 1.16 & 0.68 & 1.71 \\
35 & 1.11 & 0.81 & 0.5 & 0.0081 & 1.10 & 0.80 & 1.37 \\
40 & 1.04 & 0.92 & 0.5 & 0.0081 & 1.03 & 0.91 & 1.13 \\
45 & 0.99 & 1.03 & 0.5 & 0.0081 & 0.98 & 1.02 & 0.96 \\
\hline
\end{tabular}

Table 32: Laboratory lift and drag data for the Marlin $\left(0-45^{\circ}\right)$

\begin{tabular}{|c|cccccccc|}
\hline $\begin{array}{c}\boldsymbol{\alpha} \\
\mathbf{(}{ }^{\circ}\end{array}$ & Lift (N) & Drag (N) & $\begin{array}{c}\text { Drag - } \\
\text { board (N) }\end{array}$ & $\mathbf{U}(\mathbf{m p s})$ & $\mathbf{A}\left(\mathbf{m}^{2}\right)$ & $\mathbf{C}_{\mathrm{L}}$ & $\mathbf{C}_{\mathrm{D}}$ & $\mathbf{C}_{\mathrm{L}} / \mathbf{C}_{\mathrm{D}}$ \\
\hline 0 & 0.02 & 0.48 & 0.04 & 0.5 & 0.0035 & 0.05 & 0.09 & 0.56 \\
5 & 0.21 & 0.51 & 0.07 & 0.5 & 0.0035 & 0.49 & 0.16 & 3.00 \\
10 & 0.38 & 0.55 & 0.11 & 0.5 & 0.0035 & 0.87 & 0.25 & 3.49 \\
15 & 0.46 & 0.61 & 0.17 & 0.5 & 0.0035 & 1.06 & 0.40 & 2.64 \\
20 & 0.49 & 0.67 & 0.23 & 0.5 & 0.0035 & 1.14 & 0.53 & 2.13 \\
25 & 0.47 & 0.72 & 0.28 & 0.5 & 0.0035 & 1.08 & 0.64 & 1.68 \\
30 & 0.42 & 0.76 & 0.32 & 0.5 & 0.0035 & 0.97 & 0.73 & 1.33 \\
35 & 0.41 & 0.81 & 0.37 & 0.5 & 0.0035 & 0.94 & 0.86 & 1.09 \\
40 & 0.42 & 0.89 & 0.45 & 0.5 & 0.0035 & 0.96 & 1.04 & 0.93 \\
45 & 0.41 & 0.91 & 0.47 & 0.5 & 0.0035 & 0.94 & 1.10 & 0.86 \\
\hline
\end{tabular}


Table 33 Raw lift and drag data (minimum, maximum) collected from Trial 1 and Trial 2 in the laboratory for all nine fins

\begin{tabular}{|c|c|c|c|c|c|c|c|c|c|}
\hline & & \multicolumn{4}{|c|}{ Trial 1} & \multicolumn{4}{|c|}{ Trial 2} \\
\hline & \multirow[b]{2}{*}{ AOA } & \multicolumn{2}{|c|}{ Lift (N) } & \multicolumn{2}{|c|}{ Drag (N) } & \multicolumn{2}{|c|}{ Lift (N) } & \multicolumn{2}{|c|}{ Drag (N) } \\
\hline & & $\min$ & $\max$ & $\min$ & $\max$ & $\min$ & $\max$ & $\min$ & $\max$ \\
\hline \multirow{10}{*}{ 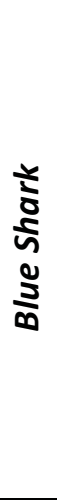 } & 0 & 0.00 & 0.00 & 0.36 & 0.46 & 0.00 & 0.02 & 0.44 & 0.51 \\
\hline & 5 & 0.18 & 0.32 & 0.44 & 0.50 & 0.16 & 0.30 & 0.45 & 0.55 \\
\hline & 10 & 0.38 & 0.50 & 0.44 & 0.56 & 0.40 & 0.58 & 0.50 & 0.60 \\
\hline & 15 & 0.44 & 0.68 & 0.52 & 0.64 & 0.52 & 0.76 & 0.50 & 0.69 \\
\hline & 20 & 0.54 & 0.72 & 0.64 & 0.76 & 0.56 & 0.80 & 0.60 & 0.71 \\
\hline & 25 & 0.54 & 0.72 & 0.68 & 0.88 & 0.56 & 0.80 & 0.65 & 0.90 \\
\hline & 30 & 0.52 & 0.64 & 0.76 & 0.90 & 0.52 & 0.68 & 0.70 & 0.90 \\
\hline & 35 & 0.54 & 0.60 & 0.76 & 0.96 & 0.56 & 0.68 & 0.80 & 0.95 \\
\hline & 40 & 0.52 & 0.62 & 0.88 & 1.08 & 0.52 & 0.65 & 0.89 & 1.01 \\
\hline & 45 & 0.50 & 0.64 & 0.98 & 1.10 & 0.50 & 0.60 & 0.90 & 1.11 \\
\hline \multirow{10}{*}{ 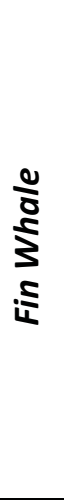 } & 0 & 0.00 & 0.05 & 0.36 & 0.45 & 0.00 & 0.02 & 0.48 & 0.60 \\
\hline & 5 & 0.22 & 0.30 & 0.40 & 0.56 & 0.12 & 0.29 & 0.50 & 0.62 \\
\hline & 10 & 0.38 & 0.48 & 0.52 & 0.64 & 0.44 & 0.56 & 0.50 & 0.68 \\
\hline & 15 & 0.48 & 0.56 & 0.56 & 0.65 & 0.44 & 0.68 & 0.59 & 0.70 \\
\hline & 20 & 0.50 & 0.68 & 0.64 & 0.76 & 0.56 & 0.76 & 0.61 & 0.80 \\
\hline & 25 & 0.50 & 0.70 & 0.62 & 0.88 & 0.55 & 0.80 & 0.60 & 0.88 \\
\hline & 30 & 0.48 & 0.65 & 0.72 & 0.92 & 0.48 & 0.68 & 0.70 & 0.89 \\
\hline & 35 & 0.47 & 0.59 & 0.72 & 0.96 & 0.48 & 0.64 & 0.75 & 0.95 \\
\hline & 40 & 0.48 & 0.54 & 0.84 & 1.00 & 0.52 & 0.63 & 0.85 & 1.05 \\
\hline & 45 & 0.49 & 0.54 & 0.85 & 1.04 & 0.56 & 0.64 & 0.95 & 1.10 \\
\hline \multirow{10}{*}{$\begin{array}{l}\frac{.}{0} \\
\frac{2}{0} \\
\frac{2}{0} \\
\frac{1}{0} \\
0\end{array}$} & 0 & 0.00 & 0.05 & 0.36 & 0.44 & 0.00 & 0.02 & 0.40 & 0.53 \\
\hline & 5 & 0.18 & 0.30 & 0.40 & 0.56 & 0.12 & 0.29 & 0.49 & 0.60 \\
\hline & 10 & 0.34 & 0.48 & 0.44 & 0.56 & 0.44 & 0.56 & 0.50 & 0.61 \\
\hline & 15 & 0.48 & 0.60 & 0.40 & 0.62 & 0.44 & 0.68 & 0.55 & 0.71 \\
\hline & 20 & 0.52 & 0.72 & 0.56 & 0.72 & 0.56 & 0.76 & 0.70 & 0.82 \\
\hline & 25 & 0.54 & 0.68 & 0.64 & 0.80 & 0.55 & 0.80 & 0.65 & 0.93 \\
\hline & 30 & 0.48 & 0.58 & 0.72 & 0.84 & 0.48 & 0.68 & 0.78 & 0.95 \\
\hline & 35 & 0.52 & 0.56 & 0.78 & 0.92 & 0.48 & 0.64 & 0.85 & 1.00 \\
\hline & 40 & 0.48 & 0.60 & 0.84 & 0.96 & 0.52 & 0.63 & 0.90 & 1.10 \\
\hline & 45 & 0.52 & 0.56 & 0.94 & 1.08 & 0.52 & 0.64 & 0.89 & 1.12 \\
\hline \multirow{6}{*}{ 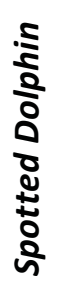 } & 0 & 0.00 & 0.10 & 0.36 & 0.44 & 0.00 & 0.00 & 0.45 & 0.51 \\
\hline & 5 & 0.26 & 0.34 & 0.47 & 0.50 & 0.20 & 0.32 & 0.49 & 0.53 \\
\hline & 10 & 0.32 & 0.42 & 0.48 & 0.52 & 0.36 & 0.52 & 0.50 & 0.60 \\
\hline & 15 & 0.38 & 0.52 & 0.48 & 0.54 & 0.52 & 0.64 & 0.55 & 0.70 \\
\hline & 20 & 0.52 & 0.60 & 0.50 & 0.58 & 0.48 & 0.72 & 0.69 & 0.81 \\
\hline & 25 & 0.60 & 0.64 & 0.54 & 0.62 & 0.52 & 0.72 & 0.70 & 0.88 \\
\hline
\end{tabular}




\begin{tabular}{|c|c|c|c|c|c|c|c|c|c|}
\hline & 30 & 0.52 & 0.62 & 0.64 & 0.74 & 0.48 & 0.68 & 0.75 & 0.90 \\
\hline & 35 & 0.50 & 0.55 & 0.70 & 0.84 & 0.44 & 0.64 & 0.85 & 1.00 \\
\hline & 40 & 0.48 & 0.50 & 0.74 & 0.88 & 0.44 & 0.56 & 0.90 & 1.05 \\
\hline & 45 & 0.46 & 0.50 & 0.84 & 0.94 & 0.44 & 0.56 & 0.90 & 1.09 \\
\hline \multirow{10}{*}{ 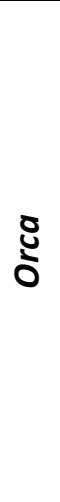 } & 0 & 0.00 & 0.04 & 0.36 & 0.42 & 0.00 & 0.00 & 0.40 & 0.57 \\
\hline & 5 & 0.20 & 0.30 & 0.40 & 0.48 & 0.12 & 0.28 & 0.49 & 0.59 \\
\hline & 10 & 0.32 & 0.46 & 0.42 & 0.60 & 0.36 & 0.52 & 0.50 & 0.65 \\
\hline & 15 & 0.40 & 0.62 & 0.48 & 0.60 & 0.40 & 0.64 & 0.55 & 0.71 \\
\hline & 20 & 0.45 & 0.65 & 0.60 & 0.68 & 0.52 & 0.68 & 0.65 & 0.72 \\
\hline & 25 & 0.48 & 0.68 & 0.65 & 0.73 & 0.55 & 0.72 & 0.75 & 0.85 \\
\hline & 30 & 0.42 & 0.60 & 0.64 & 0.82 & 0.52 & 0.64 & 0.75 & 0.90 \\
\hline & 35 & 0.50 & 0.54 & 0.72 & 0.86 & 0.44 & 0.64 & 0.80 & 0.95 \\
\hline & 40 & 0.48 & 0.54 & 0.80 & 0.90 & 0.44 & 0.60 & 0.80 & 1.03 \\
\hline & 45 & 0.48 & 0.52 & 0.88 & 0.96 & 0.44 & 0.61 & 0.90 & 1.10 \\
\hline \multirow{10}{*}{ 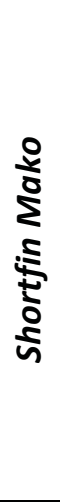 } & 0 & 0.00 & 0.04 & 0.44 & 0.45 & 0.00 & 0.01 & 0.44 & 0.46 \\
\hline & 5 & 0.22 & 0.31 & 0.46 & 0.54 & 0.24 & 0.30 & 0.45 & 0.52 \\
\hline & 10 & 0.44 & 0.54 & 0.48 & 0.56 & 0.34 & 0.56 & 0.48 & 0.56 \\
\hline & 15 & 0.56 & 0.68 & 0.55 & 0.60 & 0.62 & 0.71 & 0.51 & 0.64 \\
\hline & 20 & 0.62 & 0.78 & 0.64 & 0.73 & 0.62 & 0.80 & 0.65 & 0.76 \\
\hline & 25 & 0.66 & 0.82 & 0.73 & 0.85 & 0.62 & 0.81 & 0.75 & 0.84 \\
\hline & 30 & 0.60 & 0.70 & 0.76 & 0.86 & 0.56 & 0.72 & 0.76 & 0.86 \\
\hline & 35 & 0.56 & 0.64 & 0.85 & 0.93 & 0.58 & 0.69 & 0.90 & 0.96 \\
\hline & 40 & 0.56 & 0.62 & 0.96 & 1.01 & 0.60 & 0.67 & 0.98 & 1.04 \\
\hline & 45 & 0.60 & 0.62 & 1.01 & 1.06 & 0.62 & 0.65 & 1.04 & 1.06 \\
\hline \multirow{10}{*}{ 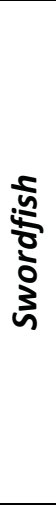 } & 0 & 0.00 & 0.02 & 0.44 & 0.52 & 0.00 & 0.04 & 0.40 & 0.54 \\
\hline & 5 & 0.12 & 0.26 & 0.42 & 0.56 & 0.24 & 0.33 & 0.49 & 0.59 \\
\hline & 10 & 0.34 & 0.48 & 0.45 & 0.58 & 0.36 & 0.52 & 0.52 & 0.69 \\
\hline & 15 & 0.46 & 0.66 & 0.55 & 0.70 & 0.44 & 0.64 & 0.60 & 0.78 \\
\hline & 20 & 0.50 & 0.58 & 0.62 & 0.72 & 0.52 & 0.68 & 0.68 & 0.83 \\
\hline & 25 & 0.50 & 0.70 & 0.75 & 0.84 & 0.52 & 0.68 & 0.74 & 0.81 \\
\hline & 30 & 0.40 & 0.68 & 0.76 & 0.92 & 0.44 & 0.56 & 0.80 & 0.85 \\
\hline & 35 & 0.48 & 0.58 & 0.85 & 0.91 & 0.40 & 0.60 & 0.85 & 0.90 \\
\hline & 40 & 0.52 & 0.52 & 0.85 & 0.92 & 0.42 & 0.56 & 0.96 & 1.00 \\
\hline & 45 & 0.43 & 0.54 & 0.90 & 0.96 & 0.42 & 0.52 & 0.97 & 1.02 \\
\hline \multirow{9}{*}{$\frac{\mathbf{s}}{\grave{\Sigma}}$} & 0 & 0.00 & 0.05 & 0.38 & 0.52 & 0.00 & 0.04 & 0.47 & 0.55 \\
\hline & 5 & 0.18 & 0.24 & 0.40 & 0.55 & 0.16 & 0.26 & 0.49 & 0.60 \\
\hline & 10 & 0.34 & 0.44 & 0.48 & 0.58 & 0.28 & 0.44 & 0.51 & 0.62 \\
\hline & 15 & 0.42 & 0.54 & 0.57 & 0.64 & 0.36 & 0.50 & 0.55 & 0.69 \\
\hline & 20 & 0.42 & 0.56 & 0.65 & 0.72 & 0.42 & 0.56 & 0.60 & 0.71 \\
\hline & 25 & 0.42 & 0.48 & 0.68 & 0.78 & 0.36 & 0.60 & 0.62 & 0.79 \\
\hline & 30 & 0.38 & 0.48 & 0.70 & 0.85 & 0.36 & 0.45 & 0.65 & 0.82 \\
\hline & 35 & 0.38 & 0.44 & 0.77 & 0.88 & 0.36 & 0.44 & 0.69 & 0.91 \\
\hline & 40 & 0.41 & 0.44 & 0.80 & 0.96 & 0.36 & 0.45 & 0.79 & 1.00 \\
\hline
\end{tabular}




\begin{tabular}{|c|c|c|c|c|c|c|c|c|c|}
\hline & 45 & 0.40 & 0.43 & 0.84 & 0.96 & 0.35 & 0.44 & 0.85 & 1.00 \\
\hline \multirow{10}{*}{ 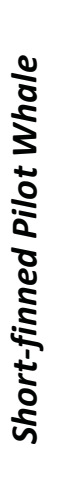 } & 0 & 0.00 & 0.04 & 0.40 & 0.48 & 0.00 & 0.04 & 0.42 & 0.59 \\
\hline & 5 & 0.18 & 0.28 & 0.44 & 0.53 & 0.08 & 0.30 & 0.50 & 0.62 \\
\hline & 10 & 0.40 & 0.52 & 0.46 & 0.56 & 0.52 & 0.64 & 0.55 & 0.69 \\
\hline & 15 & 0.56 & 0.70 & 0.48 & 0.56 & 0.68 & 0.92 & 0.60 & 0.72 \\
\hline & 20 & 0.76 & 0.82 & 0.60 & 0.68 & 0.68 & 1.05 & 0.69 & 0.85 \\
\hline & 25 & 0.84 & 0.86 & 0.76 & 0.88 & 0.76 & 1.08 & 0.85 & 1.00 \\
\hline & 30 & 0.72 & 0.82 & 0.80 & 0.92 & 0.76 & 0.96 & 0.90 & 1.08 \\
\hline & 35 & 0.72 & 0.76 & 0.84 & 0.96 & 0.72 & 0.88 & 0.99 & 1.11 \\
\hline & 40 & 0.66 & 0.78 & 0.92 & 1.04 & 0.72 & 0.84 & 1.00 & 1.17 \\
\hline & 45 & 0.60 & 0.72 & 1.08 & 1.20 & 0.72 & 0.85 & 1.09 & 1.22 \\
\hline
\end{tabular}

